\title{
Health Assessment Measurements Quality Assurance Program: Exercise 4 Final Report
}

\author{
Charles A. Barber \\ Carolyn Q. Burdette \\ Hugh V. Hayes \\ Melissa M. Phillips \\ Catherine A. Rimmer \\ Laura J. Wood \\ Lee $\mathrm{Yu}$ \\ Shaun P. Kotowski
}

This publication is available free of charge from: https://doi.org/10.6028/NIST.IR.8308

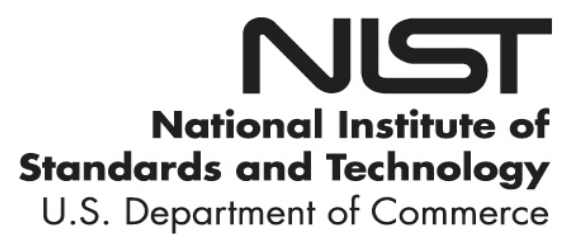




\title{
Health Assessment Measurements Quality Assurance Program: Exercise 4 Final Report
}

\author{
Charles A. Barber \\ Carolyn Q. Burdette \\ Hugh V. Hayes \\ Melissa M. Phillips \\ Catherine A. Rimmer \\ Laura J. Wood \\ Lee Yu \\ Shaun P. Kotowski \\ Chemical Sciences Division \\ Material Measurement Laboratory
}

This publication is available free of charge from:

https://doi.org/10.6028/NIST.IR.8308

May 2020

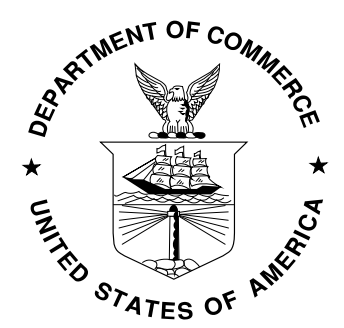

U.S. Department of Commerce

Wilbur L. Ross, Jr., Secretary

National Institute of Standards and Technology Walter Copan, NIST Director and Undersecretary of Commerce for Standards and Technology 
Certain commercial entities, equipment, or materials may be identified in this document in order to describe an experimental procedure or concept adequately. Such identification is not intended to imply recommendation or endorsement by the National Institute of Standards and Technology, nor is it intended to imply that the entities, materials, or equipment are necessarily the best available for the purpose.

National Institute of Standards and Technology Interagency or Internal Report 8308 Natl. Inst. Stand. Technol. Interag. Intern. Rep. 8308, 155 pages (May 2020)

This publication is available free of charge from: https://doi.org/10.6028/NIST.IR.8308 


\section{TABLE OF CONTENTS}

LIST OF ACRONYMS ........................................................................................................ 1

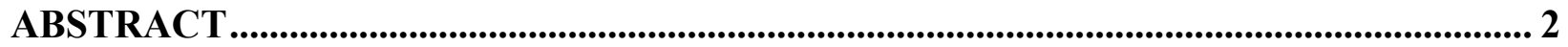

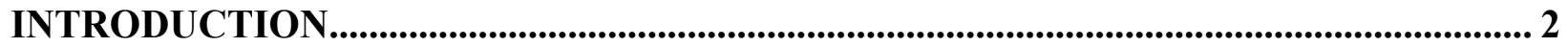

OVERVIEW OF DATA TREATMENT AND REPRESENTATION ...................................... 4

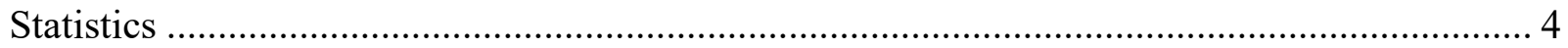

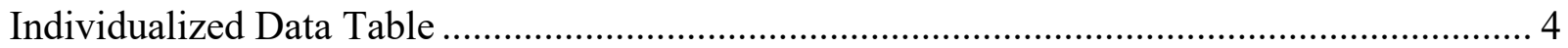

Summary Data Table

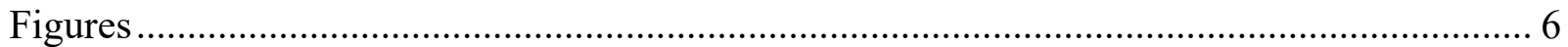

Data Summary View (Method Comparison Data Summary View) ………………............... 6

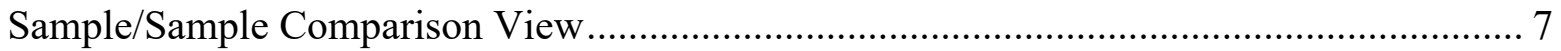

SECTION 1: NUTRITIONAL ELEMENTS (Calcium, Potassium, and Sodium) ................... 8

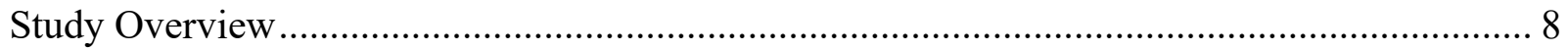

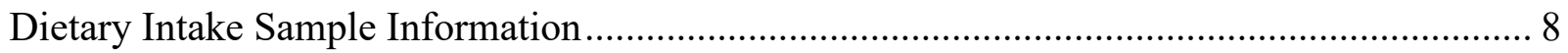

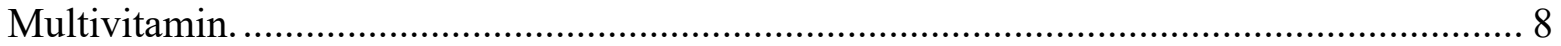

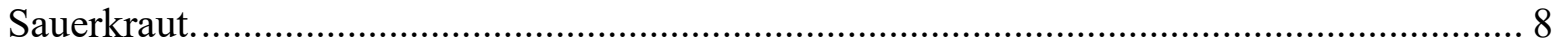

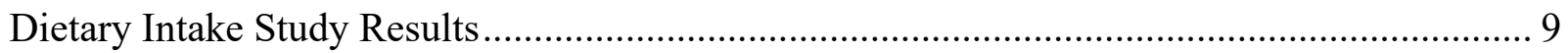

Dietary Intake Technical Recommendations .............................................................. 10

Table 1-1. Individualized data summary table (NIST) for nutritional elements in sauerkraut

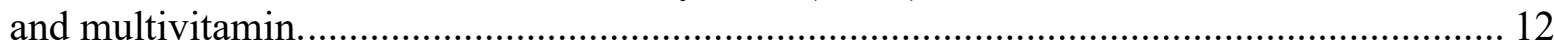

Table 1-2. Data summary table for calcium in multivitamin and sauerkraut.................... 13

Figure 1-1. Calcium in Multivitamin (data summary view - sample preparation method). 14

Figure 1-2. Calcium in Sauerkraut (data summary view - sample preparation method).... 15

Figure 1-3. Calcium in Multivitamin (data summary view - analytical method)............... 16

Figure 1-4. Calcium in Sauerkraut (data summary view - analytical method)................... 17

Figure 1-5. Laboratory means for calcium in Multivitamin and Sauerkraut (sample/sample

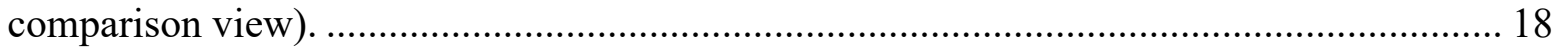

Table 1-2. Data summary table for sodium in multivitamin and sauerkraut...................... 19

Figure 1-6. Sodium in Multivitamin (data summary view - sample preparation method). 20

Figure 1-7. Sodium in Sauerkraut (data summary view - sample preparation method).... 21

Figure 1-8. Sodium in Multivitamin (data summary view - analytical method)................ 22

Figure 1-9. Sodium in Sauerkraut (data summary view - analytical method)................... 23

Figure 1-10. Laboratory means for sodium in Multivitamin and Sauerkraut (sample/sample

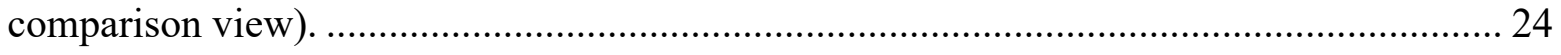

Table 1-4. Data summary table for potassium in multivitamin and sauerkraut................... 25 
Figure 1-11. Potassium in Multivitamin (data summary view - sample preparation method). 26

Figure 1-12. Potassium in Sauerkraut (data summary view - sample preparation method). 27

Figure 1-13. Potassium in Multivitamin (data summary view - analytical method)......... 28

Figure 1-14. Potassium in Sauerkraut (data summary view - analytical method)............ 29

Figure 1-15. Laboratory means for potassium in Multivitamin and Sauerkraut

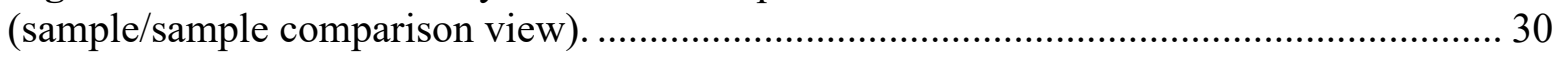

SECTION 2: TOXIC ELEMENTS (Cadmium, Lead) ........................................................ 31

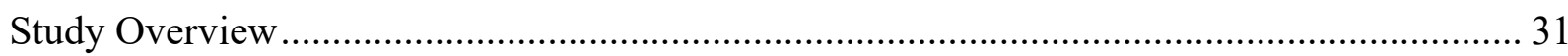

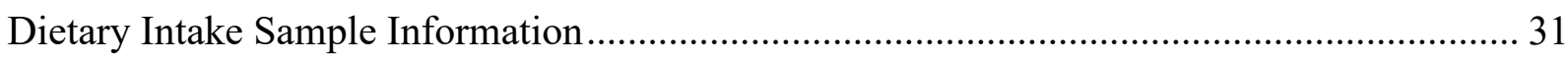

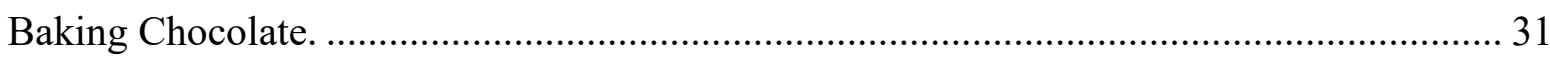

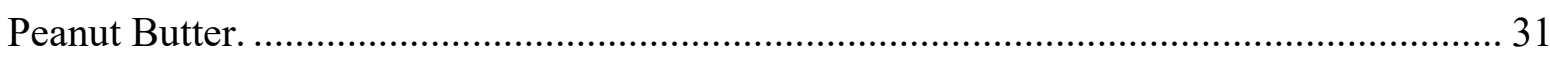

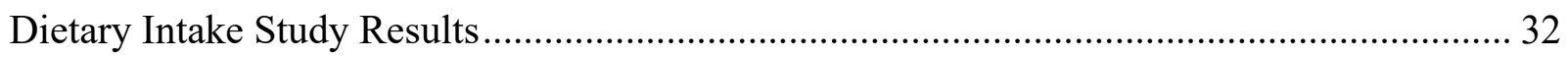

Dietary Intake Technical Recommendations ................................................................. 33

Table 2-1. Individualized data summary table (NIST) for toxic elements in baking chocolate

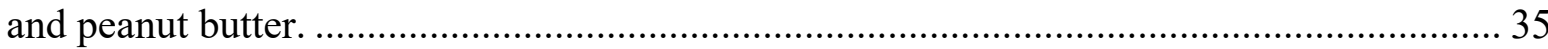

Table 2-2. Data summary table for cadmium in baking chocolate and peanut butter........ 36

Figure 2-1. Cadmium in SRM 2384 Baking Chocolate (data summary view - analytical

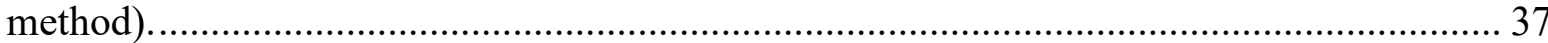

Figure 2-2. Cadmium in SRM 2384 Baking Chocolate (data summary view -sample

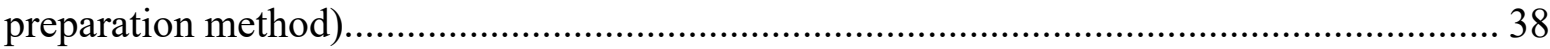

Figure 2-3. Cadmium in SRM 2387 Peanut Butter (data summary view - analytical method). 39

Figure 2-4. Cadmium in SRM 2387 Peanut Butter (data summary view -sample preparation method).

Figure 2-5. Laboratory means for cadmium in SRM 2384 Baking Chocolate and SRM 2387 Peanut Butter (sample/sample comparison view).

Table 2-3. Data summary table for lead in baking chocolate and peanut butter 42

Figure 2-6. Lead in SRM 2384 Baking Chocolate (data summary view - analytical method). 43

Figure 2-7. Lead in SRM 2384 Baking Chocolate (data summary view - sample preparation method).

Figure 2-8. Lead in SRM 2387 Peanut Butter (data summary view - analytical method). 45

Figure 2-9. Lead in SRM 2387 Peanut Butter (data summary view - sample preparation method).

Figure 2-10. Laboratory means for lead in SRM 2384 Baking Chocolate and SRM 2387 Peanut Butter (sample/sample comparison view). 
SECTION 3: WATER-SOLUBLE VITAMINS (Vitamin B $B_{12}$ ) ................................................... 48

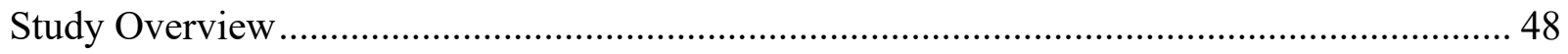

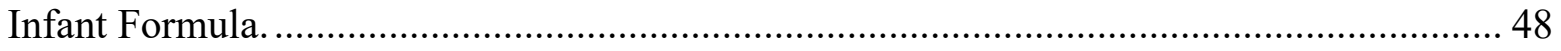

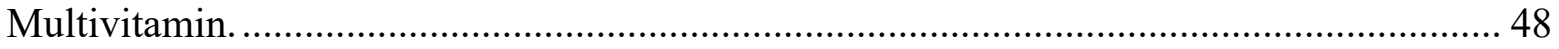

Dietary Intake Study Results....................................................................................... 49

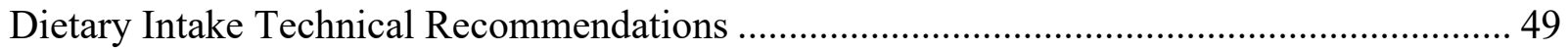

Table 3-1. Individualized data summary table (NIST) for vitamin $\mathrm{B}_{12}$ in infant formula and

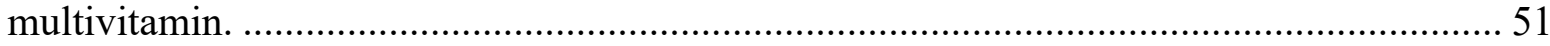

Table 3-2. Data summary table for ………………………………………………….... 52

Figure 3-1. Vitamin $B_{12}$ in SRM 1869 Infant/Adult Nutritional Formula II (data summary view - analytical method). ................................................................................................ 53

Figure 3-2. Vitamin $B_{12}$ in Multivitamin (data summary view - analytical method)........ 54

Figure 3-3. Laboratory means for Vitamin $B_{12}$ in SRM 1869 Infant/Adult Nutritional Formula II and Multivitamin (sample/sample comparison view)....................................... 55

SECTION 4: FAT-SOLUBLE VITAMINS (Vitamin $K_{1}$, Vitamin $K_{2}$ )................................... 56

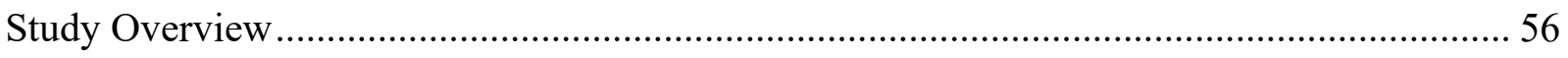

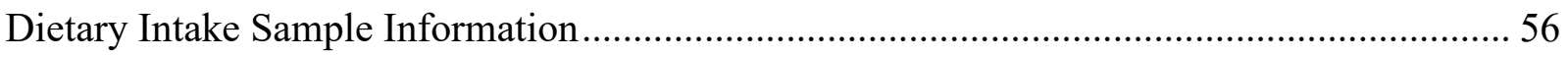

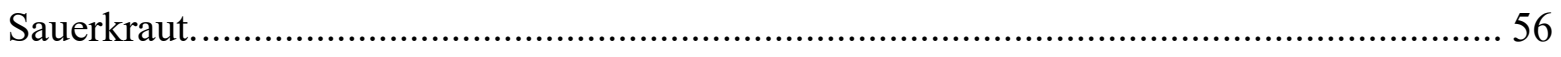

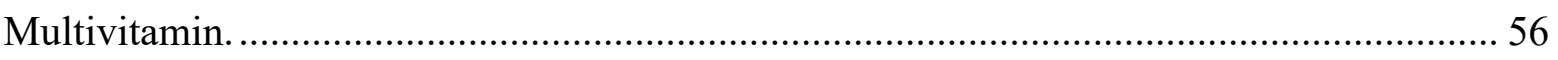

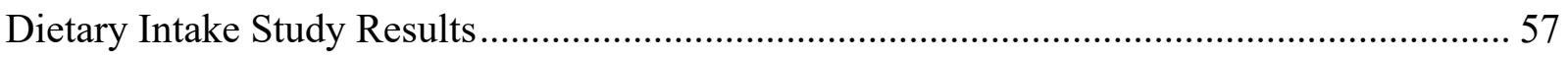

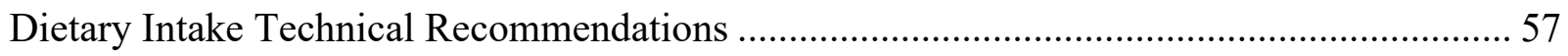

Table 4-1. Individualized data summary table (NIST) for vitamin K in sauerkraut and

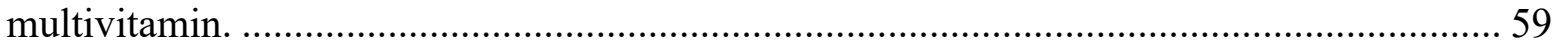

Table 4-2. Data summary table for cis-vitamin $K_{1}$ in sauerkraut and multivitamin............ 60

Table 4-3. Data summary table for trans-vitamin $K_{1}$ in sauerkraut and multivitamin. ....... 60

Table 4-4. Data summary table for total vitamin $K_{1}$ in sauerkraut and multivitamin. ........ 61

Figure 4-1. Total Vitamin $K_{1}$ in Sauerkraut (data summary view - analytical method)..... 62

Figure 4-2. Total Vitamin $K_{1}$ in Multivitamin (data summary view - analytical method). 63

Figure 4-3. Laboratory means for total vitamin $K_{1}$ in Sauerkraut and Multivitamin

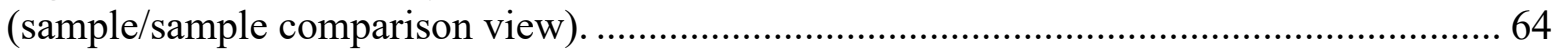

Table 4-5. Data summary table for vitamin $\mathrm{K}_{2}$ MK-4 in sauerkraut and multivitamin....... 65

Table 4-6. Data summary table for vitamin $\mathrm{K}_{2}$ MK-7 in sauerkraut and multivitamin....... 66

SECTION 5: Fatty Acids (Omega-3 and Omega-6 Fatty Acids)............................................. 67

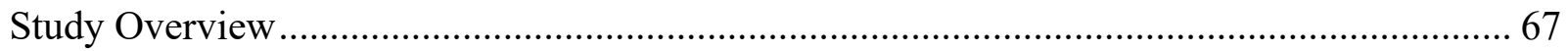

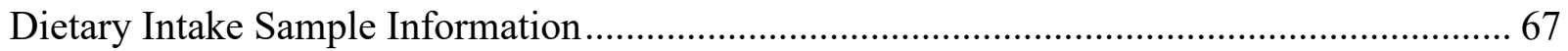

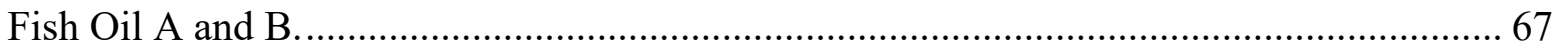




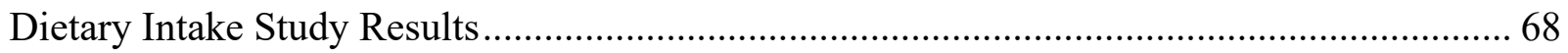

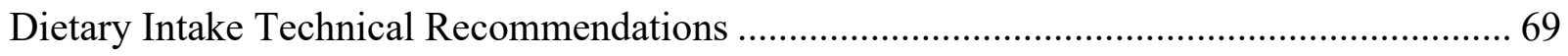

Table 5-1. Individualized data summary table (NIST) for fatty acids in fish oils............... 70

Table 5-2. Data summary table for total $\alpha$-linolenic acid in fish oil................................. 71

Figure 5-1. Total $\alpha$-linolenic acid in SRM 3275 Omega-3 and Omega-6 Fatty Acids in Fish Oil (Level 1) (data summary view - analytical method).................................................. 72

Figure 5-2. Total $\alpha$-linolenic acid in SRM 3275 Omega-3 and Omega-6 Fatty Acids in Fish Oil (Level 3) (data summary view - analytical method).................................................... 73

Figure 5-3. Laboratory means for total $\alpha$-linolenic acid in SRM 3275 Omega-3 and Omega6 Fatty Acids in Fish Oil Level 1 and Level 3 (sample/sample comparison view).............. 74

Table 5-3. Data summary table for total linoleic acid in fish oil...................................... 75

Figure 5-4. Total linoleic acid in SRM 3275 Omega-3 and Omega-6 Fatty Acids in Fish Oil (Level 1) (data summary view - analytical method).......................................................... 76

Figure 5-5. Total linoleic acid in SRM 3275 Omega-3 and Omega-6 Fatty Acids in Fish Oil (Level 3) (data summary view - analytical method)........................................................... 77

Figure 5-6. Laboratory means for total linoleic acid in SRM 3275 Omega-3 and Omega-6 Fatty Acids in Fish Oil Level 1 and Level 3 (sample/sample comparison view)................. 78

Table 5-4. Data summary table for total arachidic acid in fish oil..................................... 79

Figure 5-7. Total arachidic acid in SRM 3275 Omega-3 and Omega-6 Fatty Acids in Fish Oil (Level 1) (data summary view - analytical method)...................................................... 80

Figure 5-8. Total arachidic acid in SRM 3275 Omega-3 and Omega-6 Fatty Acids in Fish Oil (Level 3) (data summary view - analytical method). 81

Figure 5-9. Laboratory means for total arachidic acid in SRM 3275 Omega-3 and Omega-6 Fatty Acids in Fish Oil Level 1 and Level 3 (sample/sample comparison view).................. 82

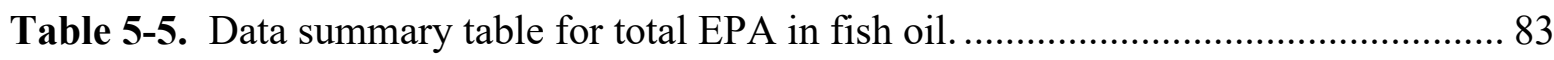

Figure 5-10. Total EPA in SRM 3275 Omega-3 and Omega-6 Fatty Acids in Fish Oil (Level

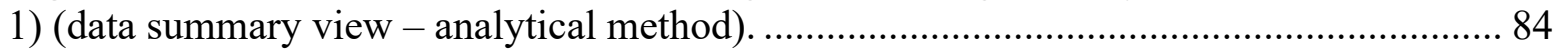

Figure 5-11. Total EPA in SRM 3275 Omega-3 and Omega-6 Fatty Acids in Fish Oil (Level 3) (data summary view - analytical method). 85

Figure 5-12. Laboratory means for total EPA in SRM 3275 Omega-3 and Omega-6 Fatty Acids in Fish Oil Level 1 and Level 3 (sample/sample comparison view)........................... 86

Table 5-6. Data summary table for total DHA in fish oil................................................. 87

Figure 5-13. Total DHA in SRM 3275 Omega-3 and Omega-6 Fatty Acids in Fish Oil (Level

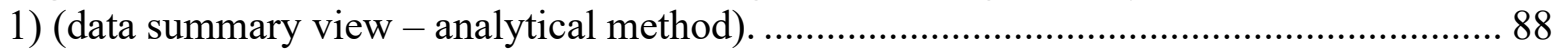

Figure 5-14. Total DHA in SRM 3275 Omega-3 and Omega-6 Fatty Acids in Fish Oil (Level 3) (data summary view - analytical method). 89

Figure 5-15. Laboratory means for total DHA in SRM 3275 Omega-3 and Omega-6 Fatty Acids in Fish Oil Level 1 and Level 3 (sample/sample comparison view). 90 


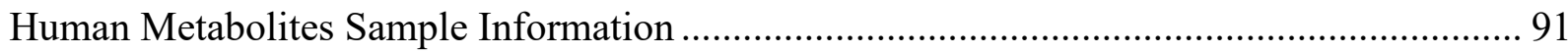

Human Serum A and B........................................................................................... 91

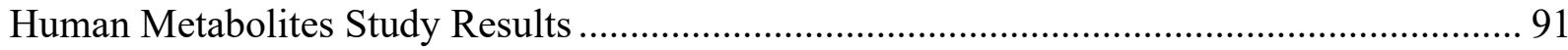

Human Metabolites Technical Recommendations............................................................. 92

Table 5-7. Individualized data summary table (NIST) for fatty acids in human serum...... 93

Table 5-8. Data summary table for total $\alpha$-linolenic acid in human serum.......................... 94

Figure 5-16. Total $\alpha$-linolenic acid in SRM 2378 Fatty Acids in Frozen Human Serum (Level

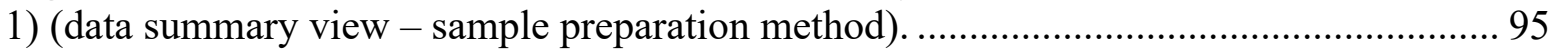

Figure 5-17. Total $\alpha$-linolenic acid in SRM 2378 Fatty Acids in Frozen Human Serum (Level 2) (data summary view - sample preparation method). ...................................................... 96

Table 5-9. Data summary table for total linoleic acid in human serum................................ 97

Figure 5-18. Total linoleic acid in SRM 2378 Fatty Acids in Frozen Human Serum (Level 1) (data summary view - sample preparation method). ..................................................... 98

Figure 5-19. Total linoleic acid in SRM 2378 Fatty Acids in Frozen Human Serum (Level 2) (data summary view - sample preparation method). ....................................................... 99

Table 5-10. Data summary table for total arachidic acid in human serum....................... 100

Table 5-11. Data summary table for total EPA in human serum........................................ 101

Figure 5-20. Total EPA in SRM 2378 Fatty Acids in Frozen Human Serum (Level 1) (data

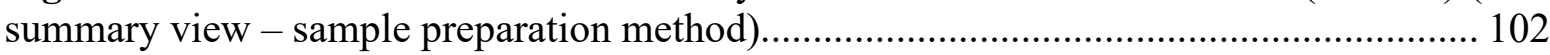

Figure 5-21. Total EPA in SRM 2378 Fatty Acids in Frozen Human Serum (Level 2) (data summary view - sample preparation method)................................................................ 103

Table 5-12. Data summary table for total DHA in human serum...................................... 104

Figure 5-22. Total DHA in SRM 2378 Fatty Acids in Frozen Human Serum (Level 1) (data summary view - sample preparation method)................................................................... 105

Figure 5-23. Total DHA in SRM 2378 Fatty Acids in Frozen Human Serum (Level 2) (data summary view - sample preparation method)............................................................... 106

Fatty Acids Overall Study Comparison …………………............................................ 107

SECTION 6: BOTANICALS (Phenolics) .................................................................................. 108

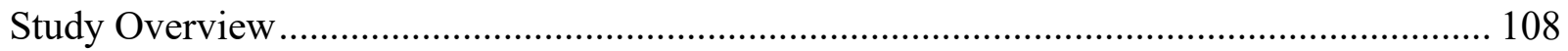

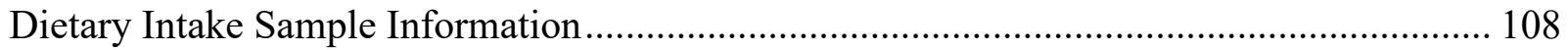

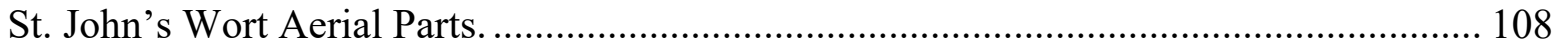

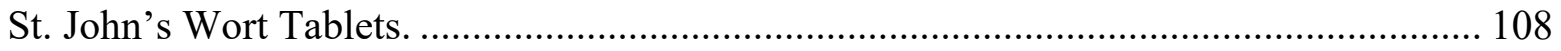

Dietary Intake Study Results....................................................................................... 109

Dietary Intake Technical Recommendations ................................................................ 110

Table 6-1. Data summary table for phenolics in St. John's Wort...................................... 112

Table 6-2. Data summary table for hyperoside in St. John's Wort ................................... 113 
Figure 6-1. Hyperoside in St. John's Wort Tablets (data summary view - analytical method) 114

Table 6-3. Data summary table for pseudohypericin in St. John's Wort. 115

Figure 6-2. Pseudohypericin in SRM 3262 St. John's Wort (Hypericum perforatum L.) Aerial Parts (data summary view - analytical method). 116

Figure 6-3. Pseudohypericin in St. John's Wort Tablets (data summary view - analytical method) 117

Table 6-4. Data summary table for quercitrin in St. John's Wort. 118

Figure 6-4. Quercitrin in SRM 3262 St. John's Wort (Hypericum perforatum L.) Aerial Parts (data summary view - analytical method).

Figure 6-5. Quercitrin in St. John's Wort Tablets (data summary view - analytical method) 120

Table 6-5. Data summary table for rutin in St. John's Wort. 121

Figure 6-6. Rutin in SRM 3262 St. John's Wort (Hypericum perforatum L.) Aerial Parts (data summary view - analytical method). 122

Figure 6-7. Rutin in St. John's Wort Tablets (data summary view - analytical method). 123

Table 6-6. Data summary table for chlorogenic acid in St. John's Wort. 124

Figure 6-8. Chlorogenic acid in SRM 3262 St. John's Wort (Hypericum perforatum L.) Aerial Parts (data summary view - analytical method). 125

Figure 6-9. Chlorogenic acid in St. John's Wort Tablets (data summary view - analytical method). 126

Table 6-7. Data summary table for adhyperforin in St. John's Wort. 127

Table 6-8. Data summary table for hyperforin in St. John's Wort. 128

Figure 6-10. Hyperforin in in SRM 3262 St. John's Wort (Hypericum perforatum L.) Aerial Parts (data summary view - analytical method). 129

Figure 6-11. Hyperforin in St. John's Wort Tablets (data summary view - analytical method) 130

Table 6-9. Data summary table for isoquercetin in St. John's Wort. 131

Figure 6-13. Isoquercetin in in SRM 3262 St. John's Wort (Hypericum perforatum) Aerial Parts (data summary view - analytical method). 132

Figure 6-14. Isoquercetin in St. John's Wort Tablets (data summary view - analytical method) 133

Table 6-10. Data summary table for quercetin in St. John's Wort. 134

Figure 6-15. Quercetin in SRM 3262 St. John's Wort (Hypericum perforatum) Aerial Parts (data summary view - analytical method). 135

Figure 6-16. Quercetin in St. John's Wort Tablets (data summary view - analytical method) 136 
SECTION 7: CONTAMINANTS (Nitrate, Nitrite)...................................................................... 137

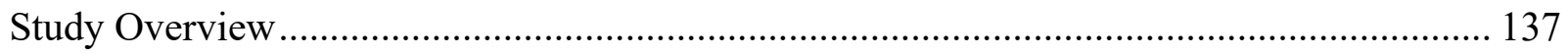

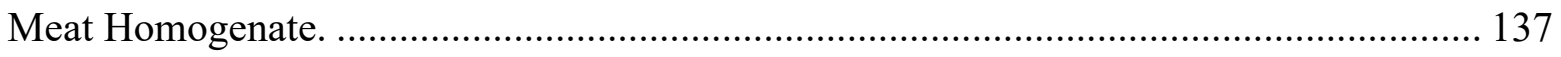

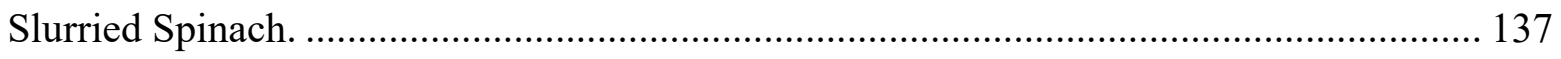

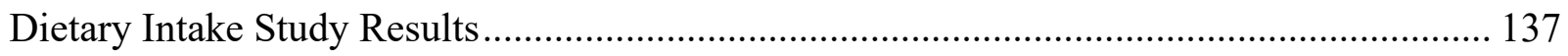

Dietary Intake Technical Recommendations ................................................................. 138

Table 7-1. Individualized data summary table (NIST) for nitrate and nitrite in meat homogenate and slurried spinach. ……...................................................................... 139

Table 7-2. Data summary table for nitrate in meat homogenate and slurried spinach. Data points highlighted in red have been flagged as potential outliers (e.g., Grubb and/or Cochran) by the NIST software package.

Figure 7-1. Nitrate in SRM 1546a Meat Homogenate (data summary view - analytical method).

Figure 7-2. Nitrate in SRM 2385 Slurried Spinach (data summary view - analytical method). 142

Table 7-3. Data summary table for nitrite in meat homogenate and slurried spinach. Data points highlighted in red have been flagged as potential outliers (e.g., Grubb and/or Cochran) by the NIST software package. 143

Figure 7-3. Nitrite in SRM 1546a Meat Homogenate (data summary view - analytical method). 144

Figure 7-3. Nitrite in SRM 2385 Slurried Spinach (data summary view - analytical method). 145 


\section{LIST OF ACRONYMS}

\begin{tabular}{|c|c|}
\hline AAS & Atomic Absorption Spectroscopy \\
\hline AI & Adequate Intake \\
\hline $\mathrm{CDC}$ & US Centers for Disease Control and Prevention \\
\hline cGMP & current Good Manufacturing Practice \\
\hline $\mathrm{COA}$ & Certificate of Analysis \\
\hline CRM & Certified Reference Material \\
\hline DNA & Deoxyribonucleic Acid \\
\hline DSQAP & Dietary Supplements Quality Assurance Program \\
\hline FDA & US Food and Drug Administration \\
\hline $\mathrm{GC}$ & Gas Chromatography \\
\hline GC-FID & Gas Chromatography with Flame Ionization Detection \\
\hline GC-MS & Gas Chromatography Mass Spectrometry \\
\hline HAMQAP & Health Assessment Measurements Quality Assurance Program \\
\hline $\mathrm{IC}$ & Ion Chromatography \\
\hline $\mathrm{IC}-\mathrm{CD}$ & Ion Chromatography with Conductivity Detection \\
\hline ICP-MS & Inductively Coupled Plasma Mass Spectrometry \\
\hline ICP-OES & Inductively Coupled Plasma Optical Emission Spectrometry \\
\hline ID ICP-MS & Isotope Dilution Inductively Coupled Plasma Mass Spectrometry \\
\hline ISE & Ion-Selective Electrode \\
\hline JCTLM & Joint Committee for Traceability in Laboratory Medicine \\
\hline FAMEs & Fatty Acid Methyl Esters \\
\hline LC-absorbance & Liquid Chromatography with Absorbance Detection \\
\hline LC-fluorescence & Liquid Chromatography with Fluorescence Detection \\
\hline LC-MS & Liquid Chromatography Mass Spectrometry \\
\hline LOQ & Limit of Quantification \\
\hline NHANES & National Health and Nutrition Examination Survey \\
\hline NIST & National Institute of Standards and Technology \\
\hline $\mathrm{NIH}$ & National Institutes of Health \\
\hline ODS & Office of Dietary Supplements \\
\hline AMRM & Analytical Methods and Reference Materials \\
\hline RMP & Reference Measurement Procedure \\
\hline QAP & Quality Assurance Program \\
\hline QL & Quantification Limit \\
\hline $\mathrm{RM}$ & Reference Material \\
\hline RSD & Relative Standard Deviation \\
\hline SD & Standard Deviation \\
\hline SRM & Standard Reference Material \\
\hline
\end{tabular}




\begin{abstract}
HAMQAP was launched in collaboration with the NIH Office of Dietary Supplements (ODS) in 2017. HAMQAP was established to enable laboratories to improve the accuracy of measurements in samples that represent human intake (e.g., foods, dietary supplements, tobacco) and samples that represent human metabolism (e.g., blood, serum, plasma, urine) for demonstration of proficiency and/or compliance with various regulations. Analytes are paired where possible to represent the full spectrum of health assessment. Exercise 4 of this program offered the opportunity for laboratories to assess their in-house measurements of nutritional elements (calcium, potassium, and sodium), contaminants (cadmium and lead, nitrates and nitrites), watersoluble vitamins (vitamin $\mathrm{B}_{12}$ ), fat-soluble vitamins (vitamins $\mathrm{K}_{1}$ and $\mathrm{K}_{2}$ ), fatty acids (select omega-3 and omega-6 fatty acids), and botanicals (phenolics) in foods and dietary supplements, and corresponding biomarkers/metabolites in clinical specimens (human sera).
\end{abstract}

\title{
INTRODUCTION
}

HAMQAP was formed in 2017, in part as a collaboration with the NIH ODS and represents ongoing efforts at NIST that were supported previously via historical QAPs, including the Dietary Supplements Laboratory QAP (DSQAP), Fatty Acids in Human Serum QAP (FAQAP), Micronutrients Measurement QAP (MMQAP), and Vitamin D Metabolites QAP (VitDQAP).

HAMQAP offers the opportunity for laboratories to assess their in-house measurements of nutritional and toxic elements, fat- and water-soluble vitamins, fatty acids, active and/or marker compounds, and contaminants in samples distributed by NIST. Samples that represent human intake (e.g., food, dietary supplements, natural products) are paired with samples that represent human metabolism (e.g., blood, serum, plasma, urine) ${ }^{1}$, where possible, to represent the full spectrum of intake and metabolism for health assessment. Reports and certificates of participation are provided and may be used to demonstrate compliance with the cGMPs or to fulfill proficiency requirements established by related accreditation bodies. In addition, NIST and HAMQAP assist the ODS AMRM program at the NIH in supporting the development and dissemination of analytical tools and reference materials. In the future, results from HAMQAP exercises could be used by ODS and NIST to identify problematic matrices and analytes for which consensus-based methods of analysis would benefit the dietary supplements and clinical communities.

NIST has decades of experience in the administration of QAPs, and HAMQAP builds on the approach taken by the former DSQAP by providing a wide range of matrices and analytes. The HAMQAP design combines activities of DSQAP, FAQAP, MMQAP, and VitDQAP, and emphasizes emerging and challenging measurements in the dietary supplement, food, and clinical matrix categories. Participating laboratories are interested in evaluating in-house methods on a wide variety of challenging, real-world matrices to demonstrate that their performance is

\footnotetext{
${ }^{1}$ Human intake samples were intended for research use only and not for human consumption. Human output samples were human-source biohazardous materials capable of transmitting infectious disease. Participants were advised to handle these materials at the Biosafety Level 2 or higher as recommended for any potentially infectious human source materials by the Centers for Disease Control and Prevention (CDC) Office of Safety, Health, and Environment and the National Institutes of Health (NIH). The supplier of the source materials for the blood, serum, and/or plasma used to prepare the sample materials found the materials to be non-reactive when tested for hepatitis B surface antigen (HBsAg), human immunodeficiency virus (HIV), hepatitis C virus $(\mathrm{HCV})$, and human immunodeficiency virus 1 antigen (HIV-1Ag) by FDA licensed tests.
} 
comparable to that of the community and that their methods provide accurate results. In areas where few standard methods have been recognized, HAMQAP offers a unique tool for assessment of the quality of measurements and provides feedback about performance that can assist participants in improving laboratory operations.

This report summarizes the results from the fourth exercise of HAMQAP. Fifty-one laboratories responded to the dietary intake portion and sixteen laboratories responded to the human metabolites portion of the call for participants distributed in April 2019 (see table below). Five human metabolites studies were cancelled prior to shipment due to low enrollment. Samples were shipped to participants in August 2019 and results were returned to NIST by September 2019. This report contains the final data and information that was disseminated to the participants in May 2020.

\begin{tabular}{|c|c|c|}
\hline Study Group & Dietary Intake Study & Human Metabolites Study \\
\hline $\begin{array}{l}\text { Nutritional } \\
\text { Elements }\end{array}$ & $\begin{array}{l}\text { Calcium, Potassium, Sodium } \\
\text { Multivitamin, Sauerkraut }\end{array}$ & $\begin{array}{l}\text { Calcium, Potassium, Sodium** } \\
\text { Human Serum, Caprine Blood }\end{array}$ \\
\hline $\begin{array}{c}\text { Toxic } \\
\text { Elements }\end{array}$ & $\begin{array}{c}\text { Cadmium, Lead } \\
\text { Peanut Butter, Chocolate* }\end{array}$ & $\begin{array}{l}\text { Cadmium, Lead** } \\
\text { Caprine Blood }\end{array}$ \\
\hline $\begin{array}{l}\text { Water-Soluble } \\
\text { Vitamins }\end{array}$ & $\begin{array}{l}\text { Vitamin } \mathrm{B}_{12} \\
\text { Multivitamin, Infant Formula }\end{array}$ & $\begin{array}{c}\text { Vitamin } B_{12}, \text { Homocysteine** } \\
\text { Methylmalonic Acid, } \\
\text { Human Serum }\end{array}$ \\
\hline $\begin{array}{l}\text { Fat-Soluble } \\
\text { Vitamins }\end{array}$ & $\begin{array}{l}\text { Vitamin } \mathrm{K}_{1} \text {, Vitamin } \mathrm{K}_{2} \\
\text { Multivitamin, Sauerkraut }\end{array}$ & $\begin{array}{c}\text { Vitamin } \mathrm{K}_{4}, \text { Vitamin } \mathrm{K}_{z} * * \\
\text { Human Serum }\end{array}$ \\
\hline Fatty Acids & $\begin{array}{l}\text { Omega-3, Omega-6 } \\
\text { Fish Oil }\end{array}$ & $\begin{array}{l}\text { Omega-3, Omega-6 } \\
\text { Human Serum }\end{array}$ \\
\hline Botanicals & $\begin{array}{l}\text { Phenolics } \\
\text { St. John's Wort }\end{array}$ & Not Offered \\
\hline Contaminants & $\begin{array}{c}\text { Nitrates, Nitrites } \\
\text { Slurried Spinach, Meat Homogenate* }\end{array}$ & $\begin{array}{l}\text { Nitrates, Nitrites** } \\
\text { Human Urine }\end{array}$ \\
\hline
\end{tabular}

* Study not sponsored by the NIH ODS.

** Cancelled due to low enrollment (less than 10 laboratories registered).

Each study group is summarized in a series of tables, figures, and text, and reported by section. Within the section, each study is summarized individually, and then conclusions are drawn for the entire study group when possible. 


\section{OVERVIEW OF DATA TREATMENT AND REPRESENTATION}

Individualized data tables and certificates are provided to the participants that have submitted data in each study, in addition to this report. Examples of the data tables using NIST data are also included in each section of this report. Community tables and figures are provided using randomized laboratory codes, with identities known only to NIST and individual laboratories. The statistical approaches are outlined below for each type of data representation.

\section{$\underline{\text { Statistics }}$}

Data tables and figures throughout this report contain information about the performance of each laboratory relative to that of the other participants in this study and relative to a target around the expected result, if available. All calculations are performed in PROLab Plus (QuoData GmbH, Dresden, Germany). ${ }^{2}$ The consensus means and standard deviations are calculated according to the robust Q/Hampel method outlined in ISO 13528:2015, Annex C. ${ }^{3}$

\section{Individualized Data Table}

The data in this table is individualized to each participating laboratory and is provided to allow participants to directly compare their data to the summary statistics (consensus or community data as well as NIST certified, reference, or estimated values, when available). The upper left of the data table includes the randomized laboratory code. Example individualized data tables are included in this report using sample NIST data; participating laboratories received uniquely coded individualized data tables in a separate distribution.

Section 1 of the data table (Your Results) contains the laboratory results as reported, including the mean and standard deviation when multiple values were reported. A blank indicates that NIST does not have data on file for that laboratory for the corresponding analyte or matrix. An empty box for standard deviation indicates that the participant reported a single value or a value below the LOQ and therefore that value was not included in the calculation of the consensus data. ${ }^{3}$ Example individualized data tables are included in this report using NIST data in Section 1 to protect the identity and performance of participants.

Also included in Section 1 are two Z-scores. The first Z-score, $Z^{\prime}$ comm, is calculated with respect to the community consensus value, taking into consideration bias that may result from the uncertainty in the assigned consensus value, using the consensus mean $\left(\mathrm{x}^{*}\right)$, consensus standard deviation $\left(\mathrm{s}^{*}\right)$, and standard deviation for proficiency assessment (SDPA, $\sigma_{P T}^{2}$ ) determined from the Q/Hampel estimator:

$$
Z^{\prime}{ }_{\text {comm }}=\frac{x_{i}-x *}{\sqrt{\sigma_{P T}^{2}+s^{* 2}}}
$$

\footnotetext{
${ }^{2}$ Certain commercial equipment, instruments, or materials are identified in this certificate to adequately specify the experimental procedure. Such identification does not imply recommendation or endorsement by the National Institute of Standards and Technology, nor does it imply that the materials or equipment identified are necessarily the best available for the purpose.

${ }^{3}$ ISO 13528:2015, Statistical methods for use in proficiency testing by interlaboratory comparisons, pp. 53-54.
} 
The second Z-score, $Z_{\mathrm{NIST}}$, is calculated with respect to the target value (NIST certified, reference, or estimated value, when available), using $x_{\text {NIST }}$ and $2 * U_{95}$ (the expanded uncertainty on the certified or reference value, $U_{95}$, or twice the standard deviation of NIST or other measurements):

$$
Z_{\mathrm{NIST}}=\frac{x_{i}-x_{\mathrm{NIST}}}{2 * U_{95}}
$$

or

$$
Z_{\mathrm{NIST}}=\frac{x_{i}-x_{\mathrm{NIST}}}{2 * U_{\mathrm{NIST}}} .
$$

The significance of the $Z$-score and $Z^{\prime}$-score is as follows:

- $|Z|<2$ indicates that the laboratory result is considered to be within the community consensus range (for $Z_{\text {comm }}^{\prime}$ ) or NIST target range (for $Z_{\mathrm{NIST}}$ ).

- $2<|Z|<3$ indicates that the laboratory result is considered to be marginally different from the community consensus value (for $Z^{\prime}$ comm) or NIST target value (for $Z_{\mathrm{NIST}}$ ).

- $|Z|>3$ indicates that the laboratory result is considered to be significantly different from the community consensus value (for $Z_{\text {comm }}^{\prime}$ ) or NIST target value (for $Z_{\text {NIST). }}$.

Section 2 of the data table (Community Results) contains the consensus results, including the number of laboratories reporting more than a single quantitative value for each analyte, the mean value determined for each analyte, and a robust estimate of the standard deviation of the reported values. ${ }^{3}$ Consensus means and standard deviations are calculated using the laboratory means; if a laboratory reported a single value, the reported value is not included in determination of the consensus values. ${ }^{3}$ Additional information on calculation of the consensus mean and standard deviation can be found in the previous section.

Section 3 of the data table (Target) contains the target values for each analyte, when available. When possible, the target value is a certified value, a reference value, or a value determined at NIST. Certified values and the associated expanded uncertainty $\left(U_{95}\right)$ have been determined with two independent analytical methods at NIST, one JCTLM-recognized RMP at NIST, or by combination of a single method at NIST and results from collaborating laboratories. Reference values are assigned using NIST values obtained from the average and standard deviation of measurements made using a single analytical method at NIST, by measurements obtained from collaborating laboratories, or a combination of NIST and collaborator data. For both certified and reference values, at least six samples have been tested and duplicate preparations from the sample package have been included, allowing the uncertainty to encompass variability due to inhomogeneity within and between packaged units. For samples in which a NIST certified or reference value is not available, a NIST-assessed value may be determined at NIST using a validated method or data from a collaborating laboratory. The NIST-assessed value represents the mean of at least three replicates. For materials acquired from another interlaboratory study or proficiency testing program, the consensus value and uncertainty from the completed round is used as the target range. Within each section of this report, the exact methods for determination of the study target values are outlined in detail. 


\section{Summary Data Table}

This data table includes a summary of all reported data for a particular analyte in a particular study. Participants can compare the raw data for their laboratory to data reported by the other participating laboratories and to the consensus data. A blank indicates that the laboratory signed up and received samples for that analyte and matrix, but NIST does not have data on file for that laboratory. Data points highlighted in red have been flagged as potential outliers (e.g., difference from reference value, Grubb and/or Cochran) by the NIST software package. The SD for the target value in this table is the uncertainty ( $\left.U_{\text {NIST }}\right)$ around the target value.

\section{Figures}

Data Summary View (Method Comparison Data Summary View)

In this view, individual laboratory data (diamonds) are plotted with the individual laboratory standard deviation (rectangle). Laboratories reporting values below the LOQ are shown in this view as downward triangles beginning at the LOQ, reported as QL on the figures. Laboratories reporting values as "below LOQ" can still be successful in the study if the target value is also below the laboratory LOQ. The blue solid line represents the consensus mean, and the green shaded area represents the $95 \%$ confidence interval for the consensus mean, based on the standard error of the consensus mean. The uncertainty in the consensus mean is calculated using the equation below, based on the repeatability standard deviation $\left(s_{\mathrm{r}}\right)$, the reproducibility standard deviation $\left(s_{\mathrm{R}}\right)$, the number of participants reporting data, and the average number of replicates reported by each participant. The uncertainty about the consensus mean is independent of the range of tolerance. Where appropriate, two consensus means may be calculated for the same sample if bimodality is identified in the data. In this case, two consensus means and ranges will be displayed in the data summary view.

$$
u_{\text {mean }}=\sqrt{\frac{s_{R}^{2}-s_{r}^{2}}{n_{\text {particpants }}}+\frac{s_{R}^{2}}{n_{\text {participants }} \times n_{\text {Average Number of Replicates per Participant }}}}
$$

The red shaded region represents the target zone for "acceptable" performance, which encompasses the NIST target value bounded by twice its uncertainty ( $U_{95}$ or $U_{\text {NIST }}$ ). The solid red lines represent the range of tolerance (values that result in an acceptable $Z^{\prime}$ score, $\left|Z^{\prime}\right| \leq 2$ ). If the lower limit is below zero, the lower limit has been set to zero. In this view, the relative locations of individual laboratory data and consensus zones with respect to the target zone can be compared easily. In most cases, the target zone and the consensus zone overlap, which is the expected result. Major program goals include both reducing the size of the consensus zone and centering the consensus zone about the target value. Analysis of an appropriate reference material as part of a quality control scheme can help to identify sources of bias for laboratories reporting results that are significantly different from the target zone. In the case in which a method comparison is relevant, different colored data points may be used to identify laboratories that used a specific approach to sample preparation, analysis, or quantitation. 


\section{Sample/Sample Comparison View}

In this view, the individual laboratory results for one sample (e.g., NIST SRM with a certified, reference, or NIST-determined value; a less challenging matrix) are compared to the results for another sample (e.g., NIST SRM with a more challenging matrix; a commercial sample). The solid red box represents the target zone for the first sample (x-axis) and the second sample (y-axis), if available. The dotted blue box represents the consensus zone for the first sample (x-axis) and the second sample (y-axis). The axes of this graph are centered about the consensus mean values for each sample or control, to a limit of twice the range of tolerance (values that result in an acceptable $Z^{\prime}$ score, $\left|Z^{\prime}\right| \leq 2$ ). Depending on the variability in the data, the axes may be scaled proportionally to better display the individual data points for each laboratory. In some cases, when the consensus and target ranges have limited overlap, the solid red box may only appear partially on the graph. If the variability in the data is high (greater than $100 \% \mathrm{RSD}$ ), the dotted blue box may also only appear partially on the graph. These views emphasize trends in the data that may indicate potential calibration issues or method biases. One program goal is to identify such calibration or method biases and assist participants in improving analytical measurement capabilities. In some cases, when two equally challenging materials are provided, the same view (sample/sample comparison) can be helpful in identifying commonalities or differences in the analysis of the two materials. 


\title{
SECTION 1: NUTRITIONAL ELEMENTS (Calcium, Potassium, and Sodium)
}

\begin{abstract}
Study Overview
In this study, participants were provided with two materials for dietary intake, multivitamin tablets and sauerkraut. Participants were asked to use in-house analytical methods to determine the mass fractions $(\mathrm{mg} / \mathrm{g})$ of calcium $(\mathrm{Ca})$, potassium $(\mathrm{K})$, and sodium $(\mathrm{Na})$ in the multivitamin tablets and sauerkraut. Consumers worldwide are being urged to limit $\mathrm{Na}$ intake and increase dietary intake for minerals such as $\mathrm{Ca}$ and $\mathrm{K}$ as part of strategies to reduce chronic disease through improved nutrition. ${ }^{4,5,6}$ Accurate measurement of $\mathrm{Ca}, \mathrm{K}$, and $\mathrm{Na}$ in foods is necessary for understanding daily intake of these elements and related health outcomes. The study samples are representative of foods and supplements that contain both low and high Na concentrations, as assessment of these elements in foods is challenged throughout sample preparation and instrumental measurement.
\end{abstract}

Dietary Intake Sample Information

Multivitamin. Participants were provided with three bottles, each containing 30 multivitamin tablets. Participants were asked to store the material at controlled room temperature, between $20{ }^{\circ} \mathrm{C}$ to $25^{\circ} \mathrm{C}$, in the original unopened bottles and to prepare one sample and report one value from each bottle provided. Before use, participants were instructed to grind all 30 tablets and mix the resulting powder thoroughly prior to removal of a test portion for analysis, and to use a sample size of at least $0.4 \mathrm{~g}$. Approximate analyte levels were not reported to participants prior to the study. Target values were assigned for $\mathrm{Ca}$ and $\mathrm{K}$ using results from the manufacturer of the material. The NIST-determined values and uncertainties are provided in the table below on an as-received basis.

NIST-Determined Mass Fraction in Multivitamin (mg/g)

$\underline{\text { Analyte }}$
Calcium $(\mathrm{Ca})$
Potassium $(\mathrm{K})$

(as-received basis)

$117.0 \pm 6.0$

$48.0 \pm 4.0$

Sauerkraut. Participants were provided with one can from a single lot of commercial sauerkraut, containing $14 \mathrm{oz}(396 \mathrm{~g})$ of material. Participants were asked to store the material at controlled room temperature, between $20^{\circ} \mathrm{C}$ to $25^{\circ} \mathrm{C}$, and to prepare three samples and report three values from the can provided. Before use, participants were instructed to homogenize the contents of the can, thoroughly mix to ensure homogeneity, and to use a sample size of at least $0.5 \mathrm{~g}$. Approximate analyte levels were not reported to participants prior to the study, and target levels for $\mathrm{Ca}$, $\mathrm{Na}$, and $\mathrm{K}$ in the sauerkraut have not been determined.

\footnotetext{
${ }^{4}$ FDA Nutrition Innovation Strategy. US Food and Drug Administration. https://www.fda.gov/food/food-labeling-nutrition/fdanutrition-innovation-strategy (accessed March 2020).

${ }^{5}$ EU Salt Reduction Framework. European Commission.

https:/ec.europa.eu/health/sites/health/files/nutrition_physical_activity/docs/salt_reportl_en.pdf

(accessed March 2020)

${ }^{6}$ Sodium intake for adults and children: Guideline. World Health Organization.

https://www.who.int/nutrition/publications/guidelines/sodium_intake/en/ (accessed March 2020).
} 
Dietary Intake Study Results

- Thirty-six laboratories enrolled in this exercise and received samples to measure each of the elements. The table below lists the participation statistics for each analyte. Some of the reported values were non-quantitative (zero or below LOQ) but are included in the participation statistics.

\begin{tabular}{|c|c|c|c|}
\hline \multirow[b]{2}{*}{ Analyte } & \multirow{2}{*}{$\begin{array}{c}\frac{\text { Number of }}{\text { Laboratories }} \\
\underline{\text { Requesting Samples }}\end{array}$} & \multicolumn{2}{|c|}{$\frac{\text { Number of Laboratories Reporting Result }}{\text { (Percent Participation) }}$} \\
\hline & & Multivitamin & Sauerkraut \\
\hline Calcium $(\mathrm{Ca})$ & 36 & $27(75 \%)$ & $20(56 \%)$ \\
\hline Sodium $(\mathrm{Na})$ & 36 & $27(75 \%)$ & $21(58 \%)$ \\
\hline Potassium (K) & 36 & $28(78 \%)$ & $21(58 \%)$ \\
\hline
\end{tabular}

- The target range overlaps the consensus range for both calcium and potassium in the multivitamin (Figures 1-1, 1-3, 1-11, and 1-13).

- Some laboratories had larger than expected within-laboratory variability which may be due to sample preparation, although the between-laboratory variabilities were very good (see table below).

Between-Laboratory Variability (\% RSD)

$\begin{array}{ccc}\text { Analyte } & \text { Multivitamin } & \text { Sauerkraut } \\ \text { Calcium }(\mathrm{Ca}) & 1 \% & 3 \% \\ \text { Sodium }(\mathrm{Na}) & 3 \% & 2 \% \\ \text { Potassium }(\mathrm{K}) & 2 \% & 2 \%\end{array}$

- Most laboratories reported using either microwave digestion or hot block digestion for determination of all three analytes (see table below). The sample preparation methods reported by participating laboratories have been highlighted in Figures 1-1 and 1-2, 1-7 and 1-8, and 1-11 and 1-12 for $\mathrm{Ca}, \mathrm{Na}$, and $\mathrm{K}$, respectively.

$\underline{\text { Reported Sample }}$

Preparation Method

Microwave Digestion

Hot Block Digestion

Solvent Extraction

\section{Percent Reporting}

$\begin{array}{ccc}\frac{\mathrm{Ca}}{71 \%} & \underline{\mathrm{Na}} & \underline{\mathrm{K}} \\ 25 \% & 70 \% & 72 \% \\ 4 \% & 26 \% & 24 \% \\ & 4 \% & 4 \%\end{array}$


- Most laboratories reported using either ICP-MS or ICP-OES for determination of all three analytes (see table below). The analytical methods reported by participating laboratories have been highlighted in Figures 1-3 and 1-4, 1-8 and 1-9, and 1-13 and 1-14 for $\mathrm{Ca}, \mathrm{Na}$, and $\mathrm{K}$, respectively.

$\begin{array}{cccc}\underline{\text { Reported Analytical Method }} & \underline{\mathrm{Ca}} & \underline{\mathrm{Na}} & \underline{\mathrm{K}} \\ \text { ICP-MS } & 56 \% & 54 \% & 54 \% \\ \text { ICP-OES } & 38 \% & 36 \% & 36 \% \\ \text { AAS } & -- & 4 \% & 4 \% \\ \text { IC-CD } & 4 \% & 4 \% & 4 \% \\ \text { ID ICP-MS } & 2 \% & 2 \% & 2 \%\end{array}$

Dietary Intake Technical Recommendations

The following recommendations are based on results obtained from the participants in this study.

- No trends were observed based on the sample preparation or analytical method used.

- The digestion procedure is critical for these materials, especially the multivitamin.

- Digestion using nitric acid and a small amount of HF should be sufficient for these analytes and samples when combined with the high temperature of a microwave system.

- The majority of laboratories reported results within the target range for calcium and potassium in the multivitamin (Figures 1-1, 1-3, 1-11, and 1-13), indicating that many laboratories are using appropriate sample preparation techniques.

- Larger than normal uncertainties or within-laboratory variability may be an indication of sample processing errors. For example, analysis of aliquots from samples that were improperly ground and homogenized will yield results that are not representative of the whole material.

- When using ICP-MS, be sure to make proper use of the instrumental features.

- Many ICP-MS instruments run in pulse mode, which is more sensitive than analog mode. Instruments typically switch automatically between pulse and analog modes depending on the dynamic range in use, and therefore the instrument must be calibrated for both modes. To ensure that the calibration curve is linear in the pulse mode, consider using a narrower range of calibration points and ensure all solutions are diluted to fall within this range.

- Collision cell or reaction cell mode can be used to reduce or eliminate the interferences for $\mathrm{Ca}\left({ }^{40} \mathrm{Ar}^{+},{ }^{12} \mathrm{C}^{16} \mathrm{O}_{2},{ }^{14} \mathrm{~N}_{2}{ }^{16} \mathrm{O}^{+},{ }^{28} \mathrm{Si}^{16} \mathrm{O}^{+}\right)$and $\mathrm{K}\left({ }^{38} \mathrm{Ar}_{1} \mathrm{H}^{+},{ }^{40} \mathrm{Ar}_{1} \mathrm{H}^{+}\right)$caused by molecular ions that have the same mass-to-charge ratio.

- When using ICP-OES, monitoring more than one wavelength for each analyte helps identify interferences or background shifts due to matrix effects at a given wavelength and helps prevent bias.

- More accurate measurements can be achieved by making sure the sample concentrations fall within the middle of the calibration curve. The calibration curve must be checked for linearity.

- Contamination from the environment does not normally impact the analytical testing for these elements when good laboratory practices are followed, however analysis of low $\mathrm{Na}$ foods may 
be problematic. CRMs are available and may be used for assay validation to ensure no contamination.

- The use of appropriate calibration materials and quality assurance samples to establish that a method is in control and being performed correctly may reduce the likelihood of outlying data. Quality assurance samples can be commercially available reference materials (CRMs, SRMs, or RMs) or materials prepared in-house. 
Table 1-1. Individualized data summary table (NIST) for nutritional elements in sauerkraut and multivitamin.

\section{National Institute of Standards \& Technology}

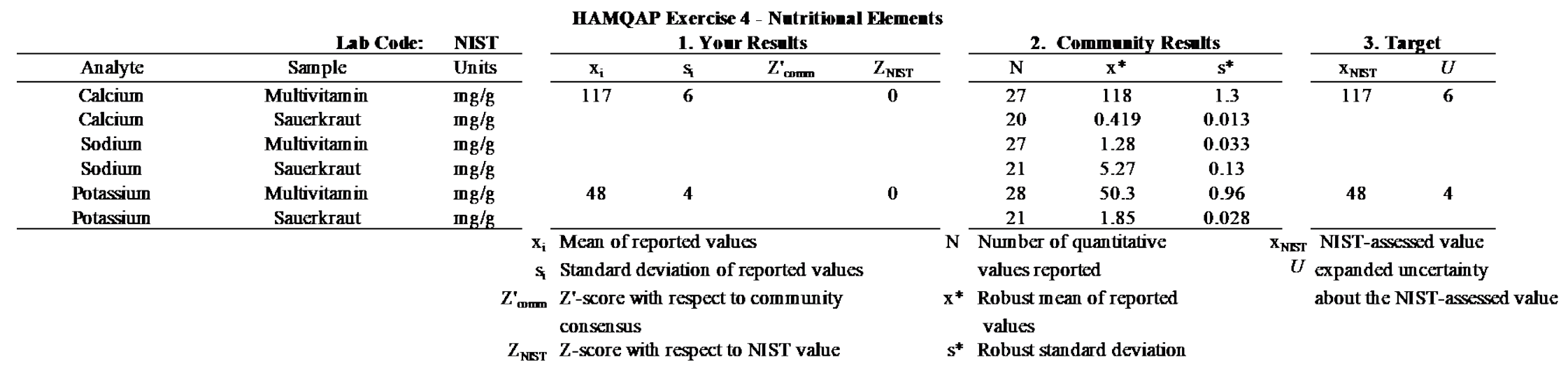


Table 1-2. Data summary table for calcium in multivitamin and sauerkraut. Data points highlighted in red have been flagged as potential outliers (e.g., Grubb and/or Cochran) by the NIST software package.

\begin{tabular}{|c|c|c|c|c|c|c|c|c|c|c|c|}
\hline & \multirow[b]{3}{*}{ Lab } & \multicolumn{10}{|c|}{ Cakium } \\
\hline & & \multicolumn{5}{|c|}{ Multivitamin (mg/g) } & \multicolumn{5}{|c|}{ Sauerkraut (mg/g) } \\
\hline & & $\mathbf{A}$ & B & $\mathbf{C}$ & Avg & SD & $\mathbf{A}$ & B & $\mathbf{C}$ & Avg & SD \\
\hline \multirow{37}{*}{ 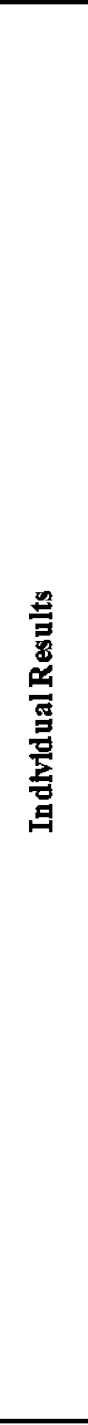 } & Target & & & & 117.0 & 6.0 & & & & & \\
\hline & D001 & 124.1 & 124.3 & 126.1 & 124.8 & 1.1 & 0.411 & 0.43 & 0.417 & 0.419 & 0.010 \\
\hline & $\mathrm{D} 002$ & 107.4 & 110.8 & 105.9 & 108.0 & 2.5 & 0.4512 & 0.4473 & 0.4165 & 0.438 & 0.019 \\
\hline & D003 & & & & & & & & & & \\
\hline & D004 & 116.25 & 114.6 & 116.52 & 115.8 & 1.0 & 0.42 & 0.43 & 0.32 & 0.390 & 0.061 \\
\hline & D005 & 140.605 & 166.78 & 171.249 & 159.5 & 16.6 & & & & & \\
\hline & D006 & 117 & 118.1 & 118 & 117.7 & 0.6 & & & & & \\
\hline & D007 & & & & & & & & & & \\
\hline & D009 & 122.3 & 131.2 & 121 & 124.8 & 5.6 & 0.397 & 0.418 & 0.424 & 0.413 & 0.014 \\
\hline & D010 & 116 & & 118 & 117.0 & 1.4 & & & & & \\
\hline & D012 & 116 & 117 & 119 & 117.3 & 1.5 & 0.452 & 0.445 & 0.43 & 0.442 & 0.011 \\
\hline & D013 & 109 & 109 & 109 & 109.0 & 0.0 & 0.471 & 0.454 & 0.5 & 0.475 & 0.023 \\
\hline & D015 & 119 & 121 & 121 & 120.3 & 1.2 & 0.464 & 0.458 & 0.458 & 0.460 & 0.003 \\
\hline & D016 & 116.32 & 116.71 & 116.39 & 116.5 & 0.2 & 0.4 & 0.39 & 0.4 & 0.397 & 0.006 \\
\hline & D017 & 119 & 125 & 110 & 118.0 & 7.5 & 0.6 & 0.6 & 0.5 & 0.567 & 0.058 \\
\hline & D018 & & & & & & & & & & \\
\hline & D019 & 113.85 & 120.12 & 114.95 & 116.3 & 3.3 & 0.08 & 0.07 & & 0.075 & 0.007 \\
\hline & D020 & 109.8 & 115.3 & 118.9 & 114.7 & 4.6 & 0.3912 & 0.4086 & 0.4027 & 0.401 & 0.009 \\
\hline & D021 & 122 & 124 & 123 & 123.0 & 1.0 & & & & & \\
\hline & D022 & 108 & 109.4 & 108.9 & 108.8 & 0.7 & 0.405 & 0.419 & 0.426 & 0.417 & 0.011 \\
\hline & D023 & 124.27 & 118.554 & 121.135 & 121.3 & 2.9 & 0.3368 & 0.3392 & 0.4632 & 0.380 & 0.072 \\
\hline & D024 & 114.61 & 111.53 & 115.46 & 113.9 & 2.1 & 0.24 & 0.23 & & 0.235 & 0.007 \\
\hline & D026 & 126.56 & 126.118 & 129.544 & 127.4 & 1.9 & 0.454 & 0.45 & 0.447 & 0.450 & 0.004 \\
\hline & D027 & & & & & & & & & & \\
\hline & D028 & 125 & 117 & 122 & 121.3 & 4.0 & 0.381 & 0.387 & 0.407 & 0.392 & 0.014 \\
\hline & D031 & 126 & 123.5 & 124.9 & 124.8 & 1.3 & & & & & \\
\hline & D032 & & & & & & & & & & \\
\hline & D033 & 114.3 & 114 & 114.2 & 114.2 & 0.2 & 0.37 & & & 0.370 & \\
\hline & D034 & & & & & & & & & & \\
\hline & D035 & 121.62 & 123.46 & 123.13 & 122.7 & 1.0 & & & & & \\
\hline & D036 & 123.06 & 124.82 & 126.4 & 124.8 & 1.7 & 0.39 & 0.41 & 0.39 & 0.397 & 0.012 \\
\hline & D038 & & & & & & & & & & \\
\hline & D045 & & & & & & & & & & \\
\hline & D046 & & & & & & & & & & \\
\hline & D047 & 112 & 113 & 112 & 112.3 & 0.6 & 0.428 & 0.412 & 0.407 & 0.416 & 0.011 \\
\hline & D049 & 623 & 119 & 119 & 287.0 & 291.0 & 0.461 & 0.462 & 0.457 & 0.460 & 0.003 \\
\hline & D050 & 118.48 & 119.398 & 120.929 & 119.6 & 1.2 & & & & & \\
\hline 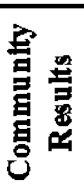 & & $\begin{array}{l}\text { Consensu } \\
\text { Consensu } \\
\text { Maximun } \\
\text { Minimum } \\
\text { N }\end{array}$ & $\begin{array}{l}\text { Mean } \\
\text { Standard }\end{array}$ & Peviation & $\begin{array}{c}118.2 \\
1.3 \\
287.0 \\
108.0 \\
27\end{array}$ & & $\begin{array}{l}\text { Consens } \\
\text { Consens } \\
\text { Maximu } \\
\text { Minimu } \\
\text { N }\end{array}$ & $\begin{array}{l}\text { Mean } \\
\text { Standard }\end{array}$ & eviation & $\begin{array}{c}0.419 \\
0.013 \\
0.567 \\
0.075 \\
19\end{array}$ & \\
\hline
\end{tabular}




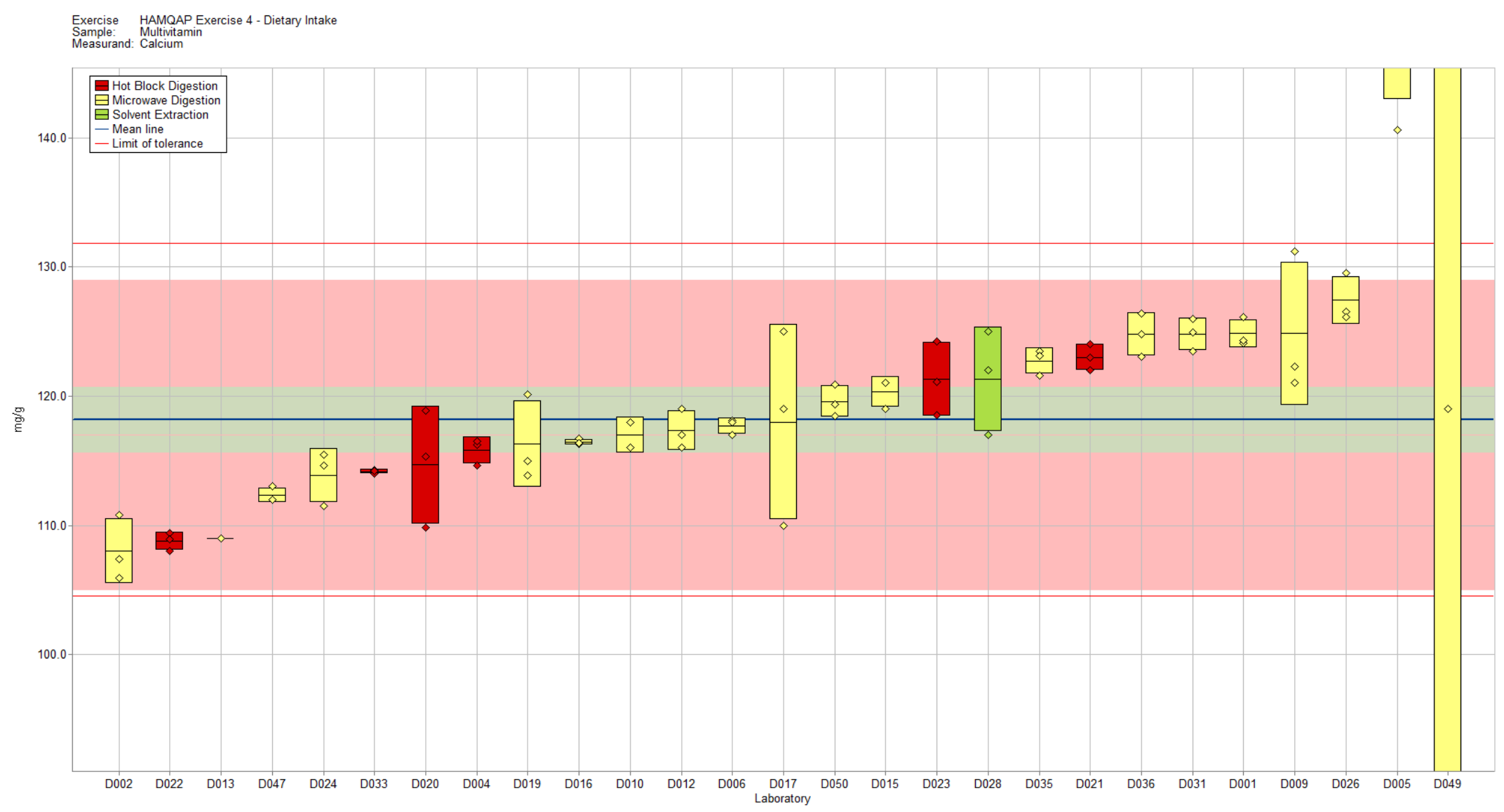

Figure 1-1. Calcium in Multivitamin (data summary view - sample preparation method). In this view, individual laboratory data are plotted (diamonds) with the individual laboratory standard deviation (rectangle). The color of the data point represents the sample preparation method employed. The solid blue line represents the consensus mean, and the green shaded region represents the $95 \%$ confidence interval for the consensus mean. The red solid lines represent the consensus range of tolerance, calculated as the values above and below the consensus mean that result in an acceptable $Z_{\text {comm }}^{\prime}$ score, $\left|Z_{\text {comm }}^{\prime}\right| \leq 2$. The red shaded region represents the NIST range of tolerance, which encompasses the target value bounded by twice its uncertainty $\left(U_{\text {NIST }}\right)$ and represents the range that results in an acceptable $Z_{\text {NIST }}$ score, $\left|Z_{\text {NIST }}\right| \leq 2$. 


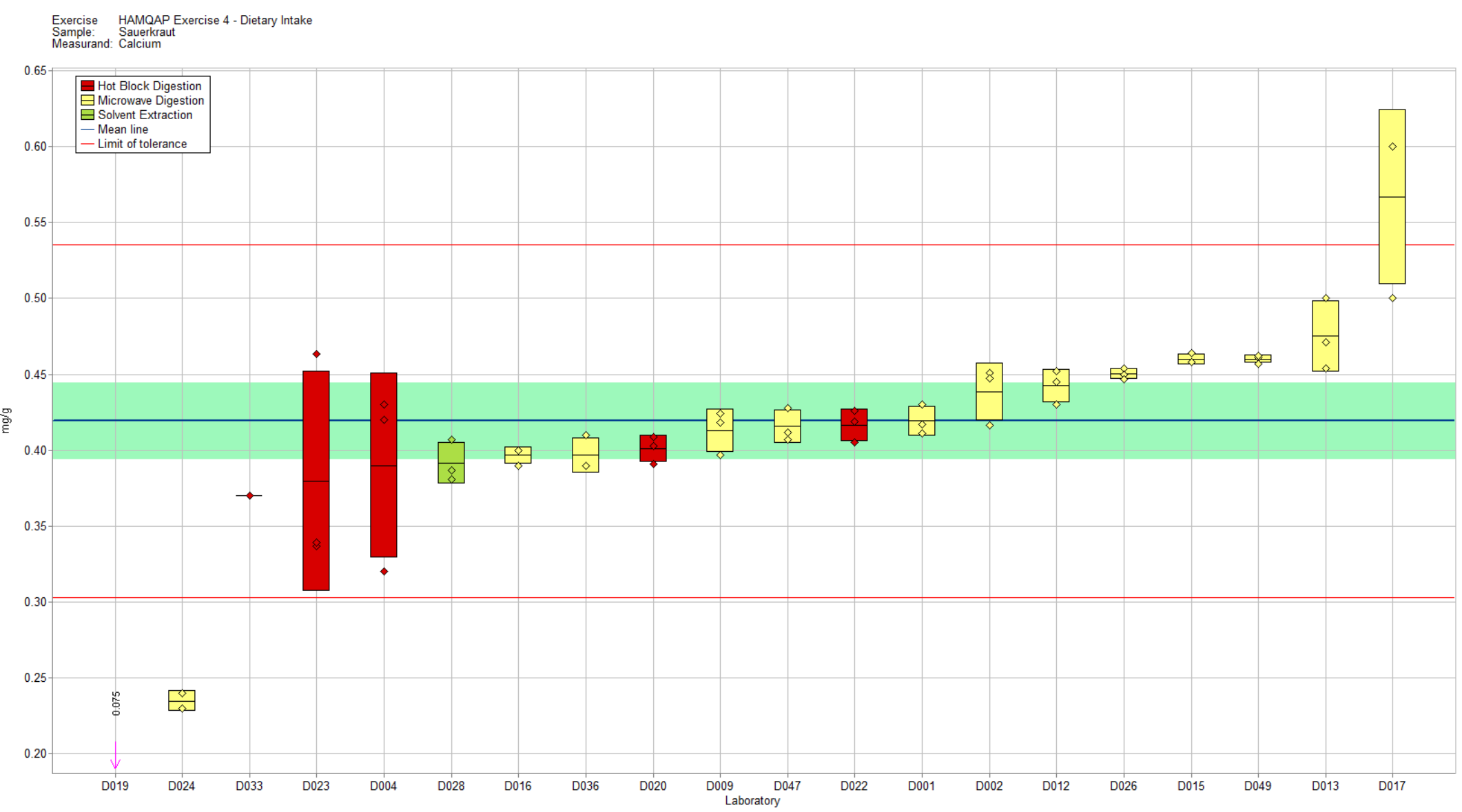

Figure 1-2. Calcium in Sauerkraut (data summary view - sample preparation method). In this view, individual laboratory data are plotted (diamonds) with the individual laboratory standard deviation (rectangle). The color of the data point represents the sample preparation method employed. The solid blue line represents the consensus mean, and the green shaded region represents the $95 \%$ confidence interval for the consensus mean. The red solid lines represent the consensus range of tolerance, calculated as the values above and below the consensus mean that result in an acceptable $Z_{\text {comm }}^{\prime}$ score, $\left|Z_{\text {comm }}^{\prime}\right| \leq 2$. A NIST value has not been determined in this material. 


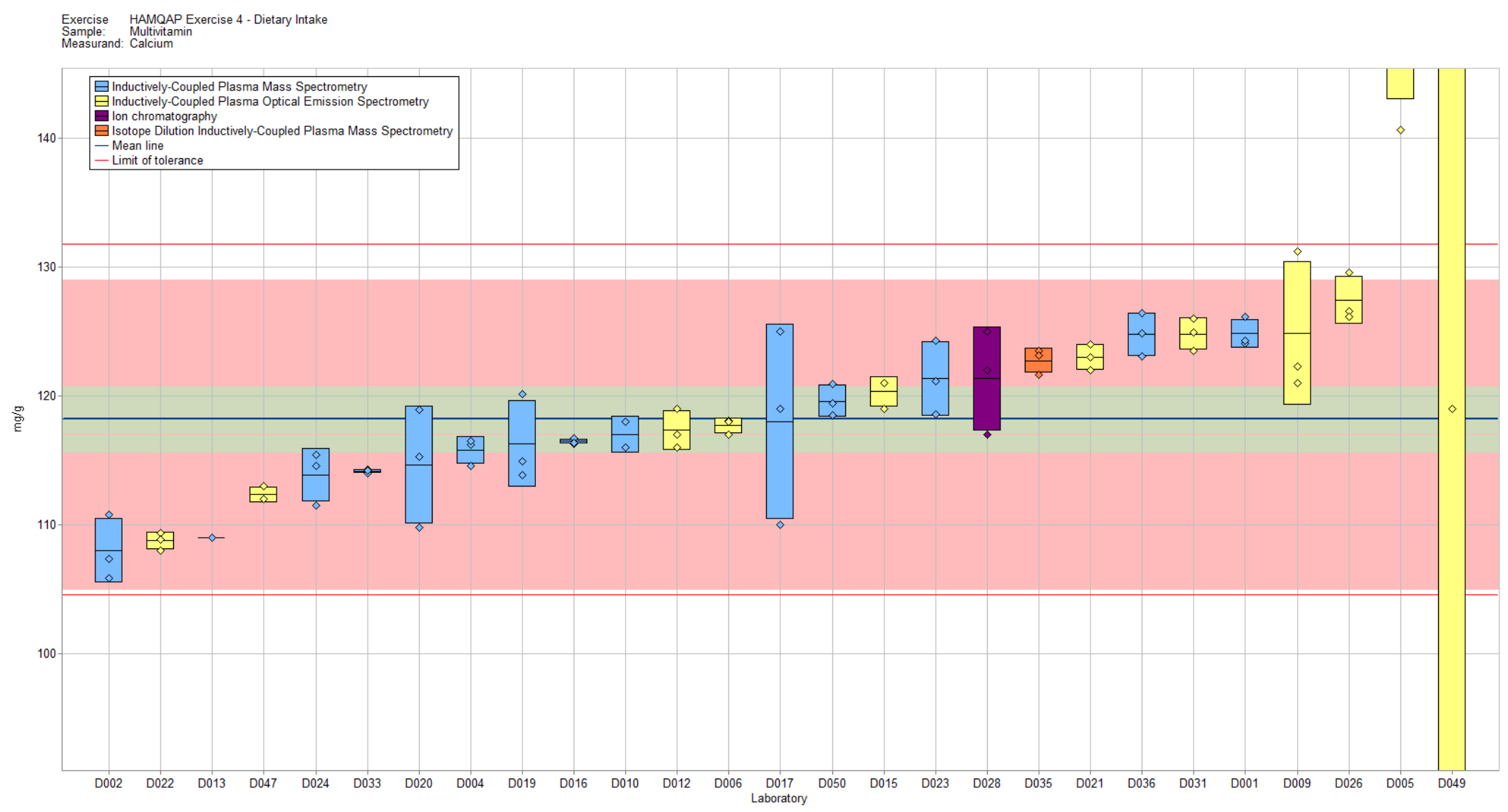

Figure 1-3. Calcium in Multivitamin (data summary view - analytical method). In this view, individual laboratory data are plotted (diamonds) with the individual laboratory standard deviation (rectangle). The color of the data point represents the analytical method employed. The solid blue line represents the consensus mean, and the green shaded region represents the $95 \%$ confidence interval for the consensus mean. The red solid lines represent the consensus range of tolerance, calculated as the values above and below the consensus mean that result in an acceptable $Z_{\text {comm }}^{\prime}$ score, $\left|Z_{\text {comm }}^{\prime}\right| \leq 2$. The red shaded region represents the NIST range of tolerance, which encompasses the target value bounded by twice its uncertainty ( $\left.U_{\mathrm{NIST}}\right)$ and represents the range that results in an acceptable $Z_{\mathrm{NIST}}$ score, $\left|Z_{\text {NIST }}\right| \leq 2$. 


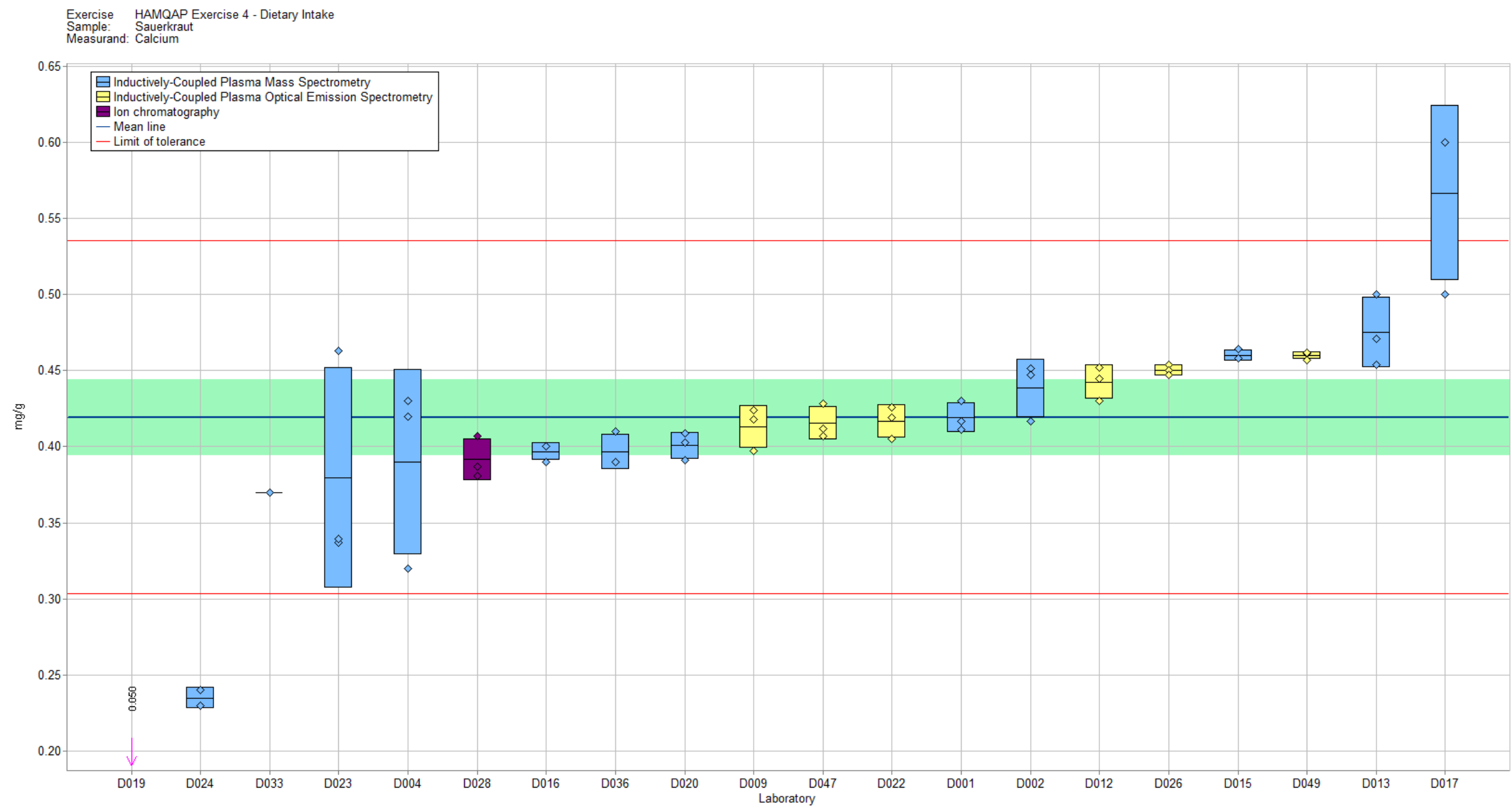

Figure 1-4. Calcium in Sauerkraut (data summary view - analytical method). In this view, individual laboratory data are plotted (diamonds) with the individual laboratory standard deviation (rectangle). The color of the data point represents the analytical method employed. The solid blue line represents the consensus mean, and the green shaded region represents the $95 \%$ confidence interval for the consensus mean. The red solid lines represent the consensus range of tolerance, calculated as the values above and below the consensus mean that result in an acceptable $Z_{\text {comm }}^{\prime}$ score, $\left|Z_{\text {comm }}^{\prime}\right| \leq 2$. A NIST value has not been determined in this material. 


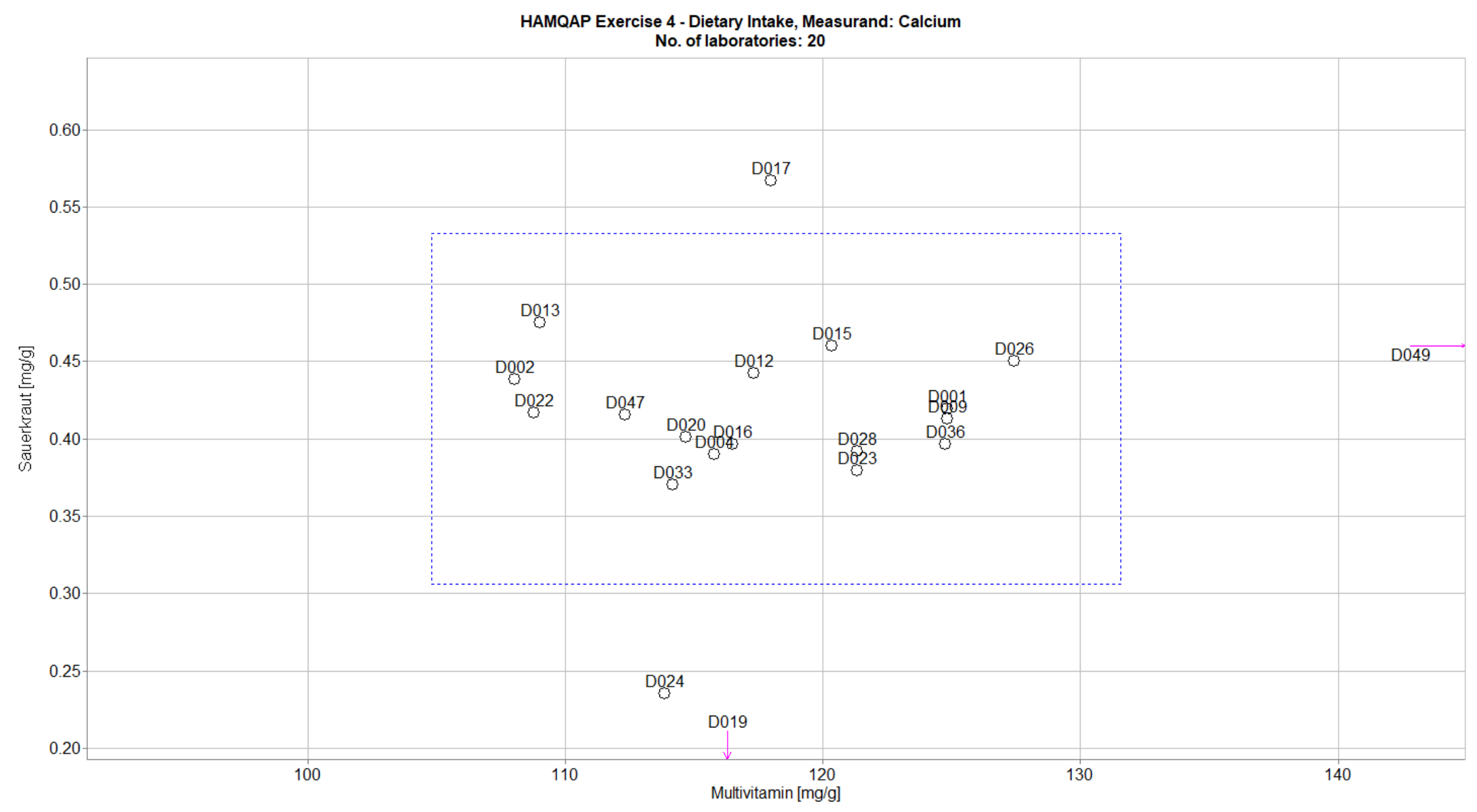

Figure 1-5. Laboratory means for calcium in Multivitamin and Sauerkraut (sample/sample comparison view). In this view, the individual laboratory mean for one sample (multivitamin) is compared to the individual laboratory mean for a second sample (sauerkraut). The dotted blue box represents the consensus range of tolerance for multivitamin (x-axis) and sauerkraut (y-axis), calculated as the values above and below the consensus means that result in an acceptable $Z_{\text {comm }}^{\prime}$ score, $\left|Z_{\text {comm }}^{\prime}\right| \leq 2$. 
Table 1-2. Data summary table for sodium in multivitamin and sauerkraut. Data points highlighted in red have been flagged as potential outliers (e.g., Grubb and/or Cochran) by the NIST software package.

\begin{tabular}{|c|c|c|c|c|c|c|c|c|c|c|c|}
\hline & \multirow[b]{3}{*}{ Lab } & \multicolumn{10}{|c|}{ Sodium } \\
\hline & & \multicolumn{5}{|c|}{ Multivitamin (mg/g) } & \multicolumn{5}{|c|}{ Sauerkraut (mg/g) } \\
\hline & & $\mathbf{A}$ & B & $\mathbf{C}$ & Avg & SD & $\mathbf{A}$ & B & $\mathbf{C}$ & Avg & SD \\
\hline \multirow{37}{*}{ 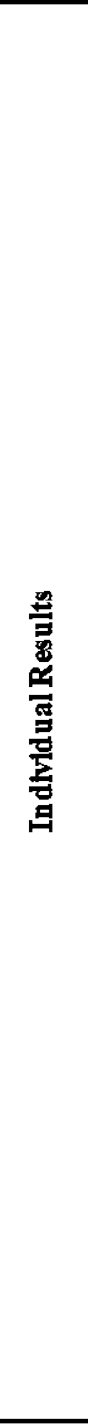 } & Target & & & & & & & & & & \\
\hline & D001 & 1.27 & 1.26 & 1.31 & 1.280 & 0.026 & 5.29 & 5.16 & 5.22 & 5.22 & 0.07 \\
\hline & $\mathrm{D} 002$ & 1.4047 & 1.4353 & 1.4609 & 1.434 & 0.028 & 4.5962 & 4.3729 & 4.7032 & 4.56 & 0.17 \\
\hline & D003 & & & & & & & & & & \\
\hline & D004 & 1.33 & 1.29 & 1.29 & 1.303 & 0.023 & 5.78 & 5.59 & 5.8 & 5.72 & 0.12 \\
\hline & D005 & 1.815 & 2.001 & 2.041 & 1.952 & 0.121 & & & & & \\
\hline & D006 & 1.39 & 1.39 & 1.42 & 1.400 & 0.017 & & & & & \\
\hline & D007 & & & & & & & & & & \\
\hline & D009 & 1.83 & 1.81 & 1.8 & 1.813 & 0.015 & 4.92 & 4.95 & 4.72 & 4.86 & 0.13 \\
\hline & D010 & 1.21 & & 1.22 & 1.215 & 0.007 & & & & & \\
\hline & D012 & 1.22 & 1.2 & 1.21 & 1.210 & 0.010 & 5.62 & 5.54 & 5.49 & 5.55 & 0.07 \\
\hline & D013 & 1.24 & 1.27 & 1.2 & 1.237 & 0.035 & 4.58 & 4.85 & 5.07 & 4.83 & 0.25 \\
\hline & D015 & 1.19 & 1.19 & 1.21 & 1.197 & 0.012 & 5.19 & 5.25 & 5.35 & 5.26 & 0.08 \\
\hline & D016 & 1.17 & 1.17 & 1.19 & 1.177 & 0.012 & 6.41 & 6.65 & 6.53 & 6.53 & 0.12 \\
\hline & D017 & 1.7 & 1.9 & 1.1 & 1.567 & 0.416 & 4.9 & 5 & 4.7 & 4.87 & 0.15 \\
\hline & D018 & & & & & & & & & & \\
\hline & D019 & 1.04 & 1.09 & 1.02 & 1.050 & 0.036 & 4.5 & 4.16 & 4.01 & 4.22 & 0.25 \\
\hline & D020 & 1.242 & 1.219 & 1.219 & 1.227 & 0.013 & 4.916 & 4.61 & 4.717 & 4.75 & 0.16 \\
\hline & D021 & 1.34 & 1.37 & 1.35 & 1.353 & 0.015 & & & & & \\
\hline & D022 & 1.4 & 1.5 & 1.48 & 1.460 & 0.053 & 5.25 & 5.34 & 5.31 & 5.30 & 0.05 \\
\hline & D023 & 1.497 & 1.532 & 1.493 & 1.507 & 0.021 & 5.2272 & 5.6384 & 5.3144 & 5.39 & 0.22 \\
\hline & D024 & 0.925 & 0.89 & 1.01 & 0.942 & 0.062 & 4.56 & 4.64 & & 4.60 & 0.06 \\
\hline & D026 & 1.455 & 1.459 & 1.464 & 1.459 & 0.005 & 5.543 & 5.709 & 5.6 & 5.62 & 0.08 \\
\hline & D027 & & & & & & & & & & \\
\hline & D028 & 1.17 & 1.18 & 1.2 & 1.183 & 0.015 & 5.25 & 5.31 & 5.32 & 5.29 & 0.04 \\
\hline & D031 & 1.288 & 1.235 & 1.276 & 1.266 & 0.028 & & & & & \\
\hline & D032 & & & & & & & & & & \\
\hline & D033 & 1.08 & 1.18 & 1.13 & 1.130 & 0.050 & 7.02 & & & 7.02 & \\
\hline & D034 & & & & & & & & & & \\
\hline & D035 & 1.2 & 1.17 & 1.18 & 1.183 & 0.015 & & & & & \\
\hline & D036 & & & & & & 5.27 & 5.36 & 5.25 & 5.29 & 0.06 \\
\hline & D038 & 1.04 & 0.87 & 1.06 & 0.990 & 0.104 & 5.15 & 5.22 & 5.17 & 5.18 & 0.04 \\
\hline & D045 & & & & & & & & & & \\
\hline & D046 & & & & & & & & & & \\
\hline & D047 & 1.24 & 1.34 & 1.22 & 1.267 & 0.064 & 6.768 & 6.616 & 6.845 & 6.74 & 0.12 \\
\hline & D049 & 5.61 & 1.21 & 1.27 & 2.70 & 2.52 & 5.45 & 5.52 & 5.5 & 5.49 & 0.04 \\
\hline & D050 & 1.199 & 1.21 & 1.227 & 1.212 & 0.014 & & & & & \\
\hline & & Consensu & Mean & & 1.277 & & Consens & Mean & & 5.27 & \\
\hline 寻 。 & & Consensu & Standard & eviation & 0.033 & & Consens & Standard & reviation & 0.13 & \\
\hline 当 焉 & & Maximun & & & 2.697 & & Maximu & & & 7.02 & \\
\hline 是 & & Minimum & & & 0.942 & & Minimu & & & 4.22 & \\
\hline & & $\mathrm{N}$ & & & 27 & & $\mathrm{~N}$ & & & 20 & \\
\hline
\end{tabular}




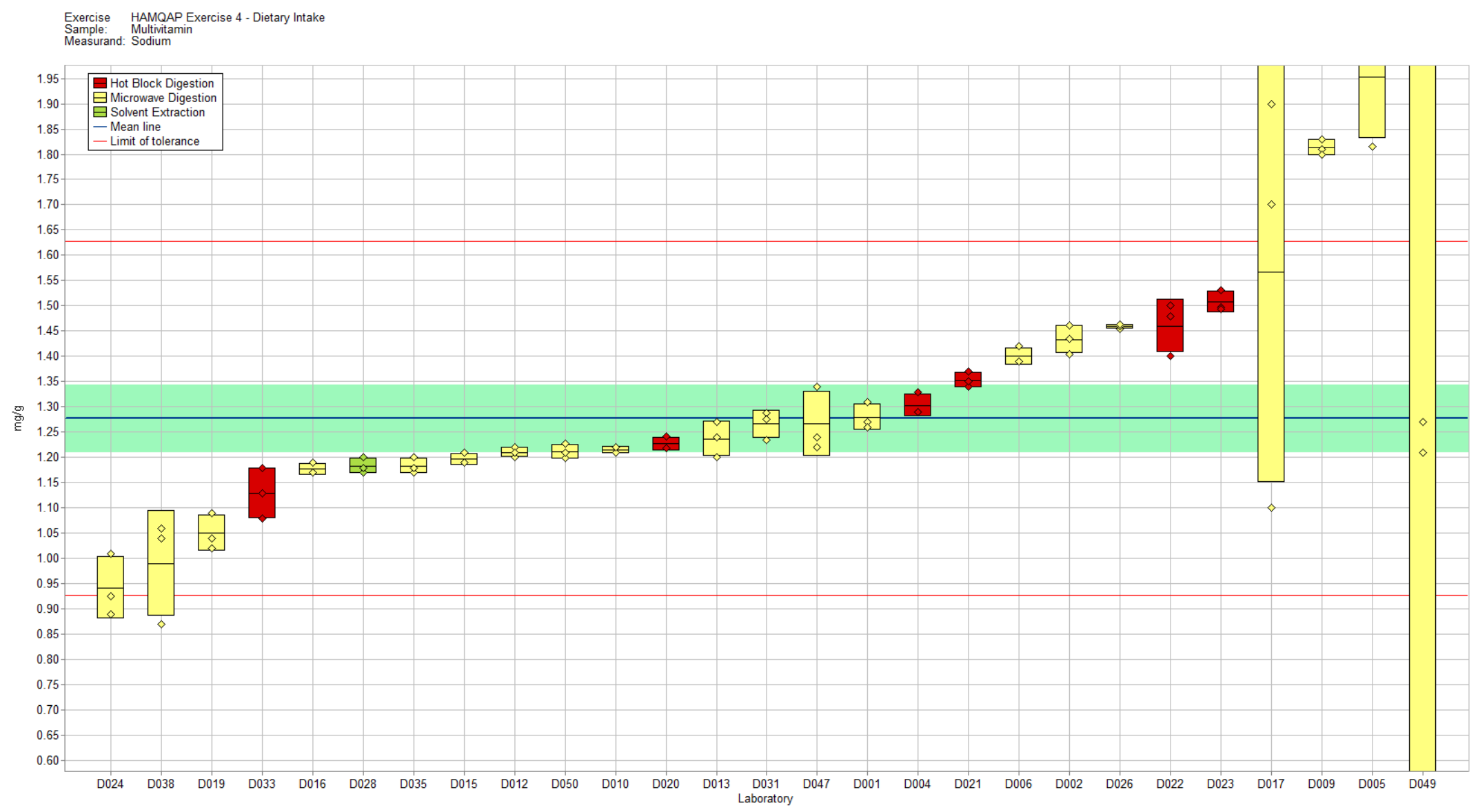

Figure 1-6. Sodium in Multivitamin (data summary view - sample preparation method). In this view, individual laboratory data are plotted (diamonds) with the individual laboratory standard deviation (rectangle). The color of the data point represents the sample preparation method employed. The solid blue line represents the consensus mean, and the green shaded region represents the $95 \%$ confidence interval for the consensus mean. The red solid lines represent the consensus range of tolerance, calculated as the values above and below the consensus mean that result in an acceptable $Z_{\text {comm }}^{\prime}$ score, $\left|Z_{\text {comm }}^{\prime}\right| \leq 2$. A NIST value has not been determined in this material. 


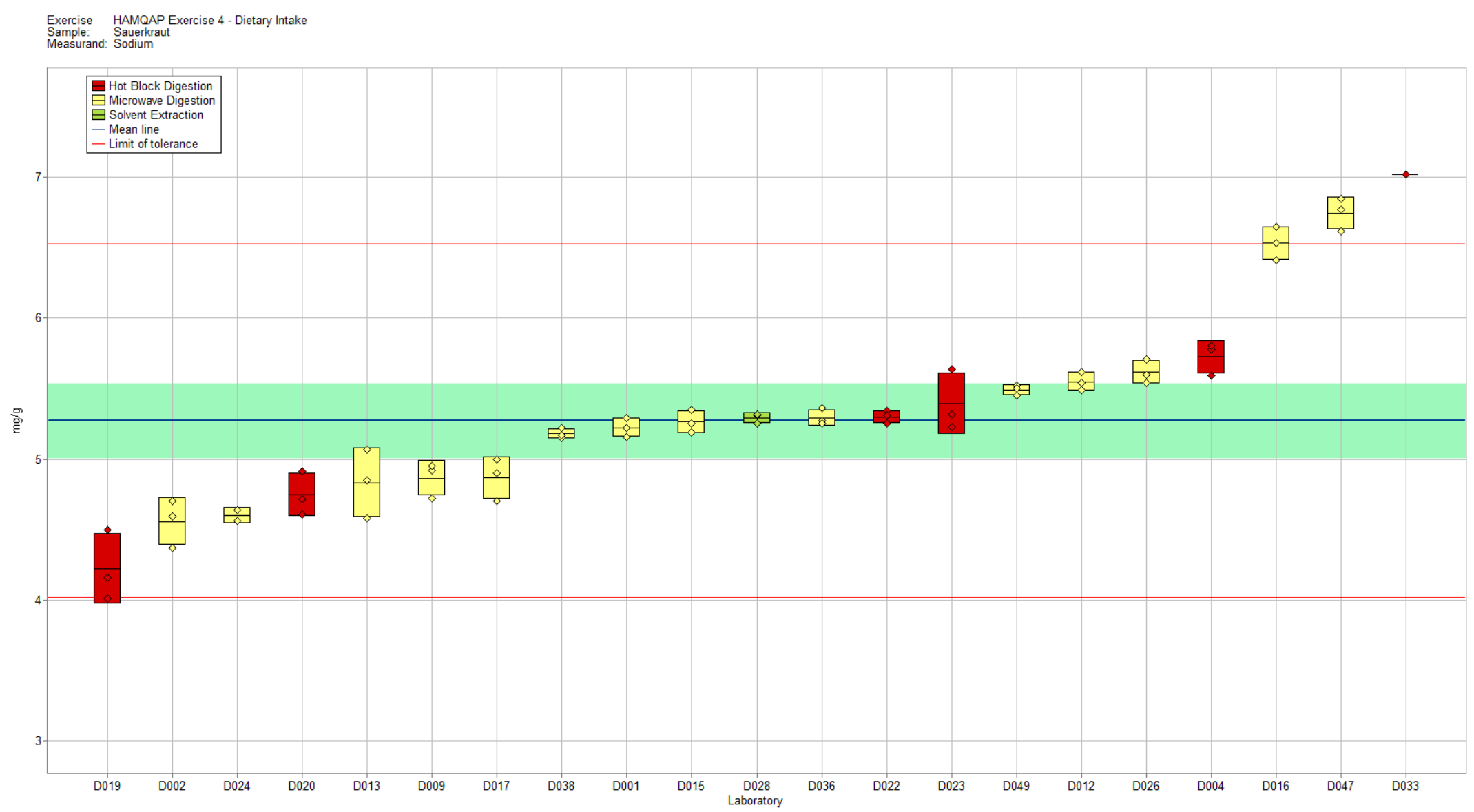

Figure 1-7. Sodium in Sauerkraut (data summary view - sample preparation method). In this view, individual laboratory data are plotted (diamonds) with the individual laboratory standard deviation (rectangle). The color of the data point represents the sample preparation method employed. The solid blue line represents the consensus mean, and the green shaded region represents the $95 \%$ confidence interval for the consensus mean. The red solid lines represent the consensus range of tolerance, calculated as the values above and below the consensus mean that result in an acceptable $Z_{\text {comm }}^{\prime}$ score, $\left|Z_{\text {comm }}^{\prime}\right| \leq 2$. A NIST value has not been determined in this material. 


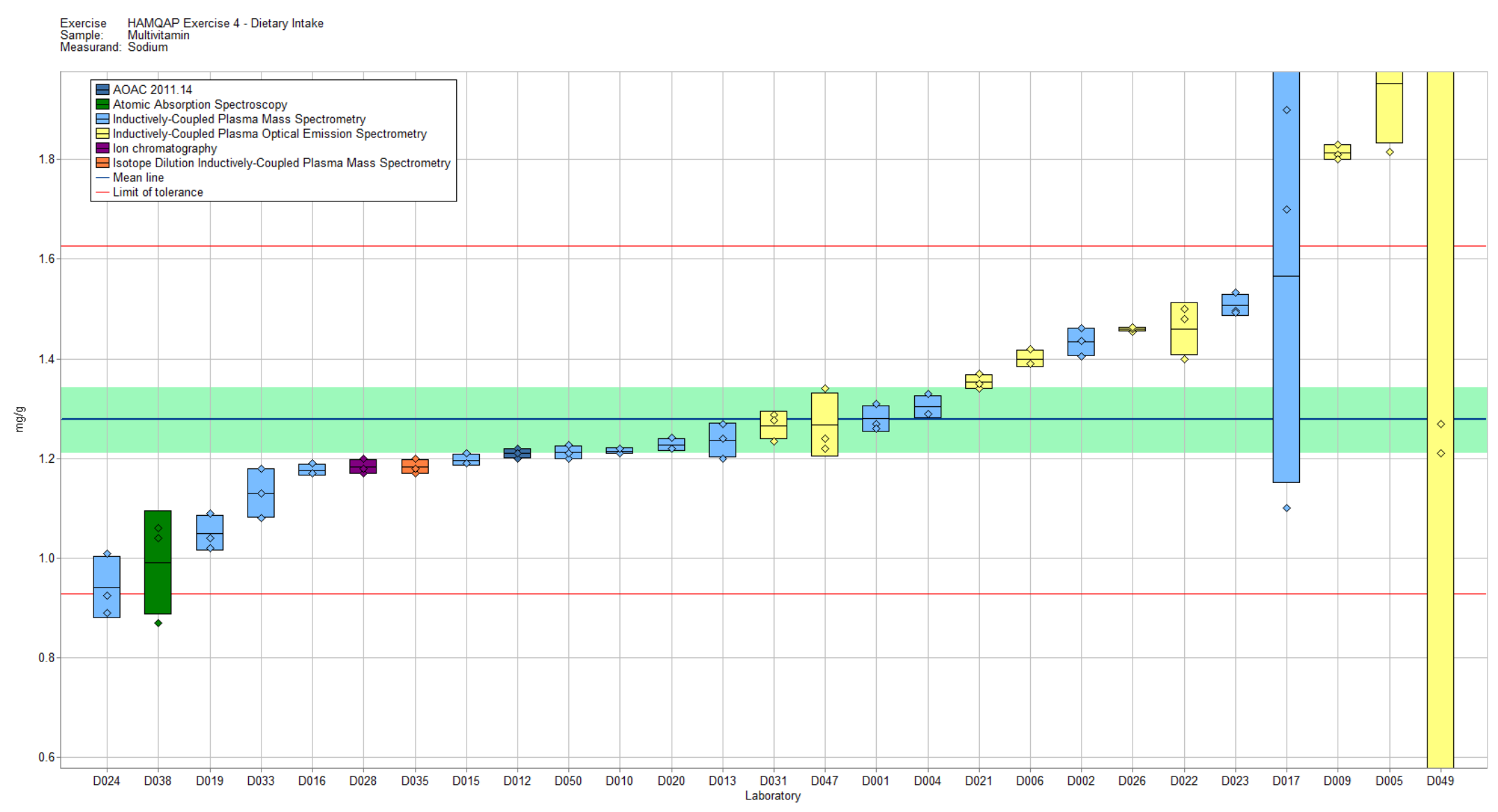

Figure 1-8. Sodium in Multivitamin (data summary view - analytical method). In this view, individual laboratory data are plotted (diamonds) with the individual laboratory standard deviation (rectangle). The color of the data point represents the analytical method employed. The solid blue line represents the consensus mean, and the green shaded region represents the $95 \%$ confidence interval for the consensus mean. The red solid lines represent the consensus range of tolerance, calculated as the values above and below the consensus mean that result in an acceptable $Z_{\text {comm }}^{\prime}$ score, $\left|Z_{\text {comm }}^{\prime}\right| \leq 2$. A NIST value has not been determined in this material. 


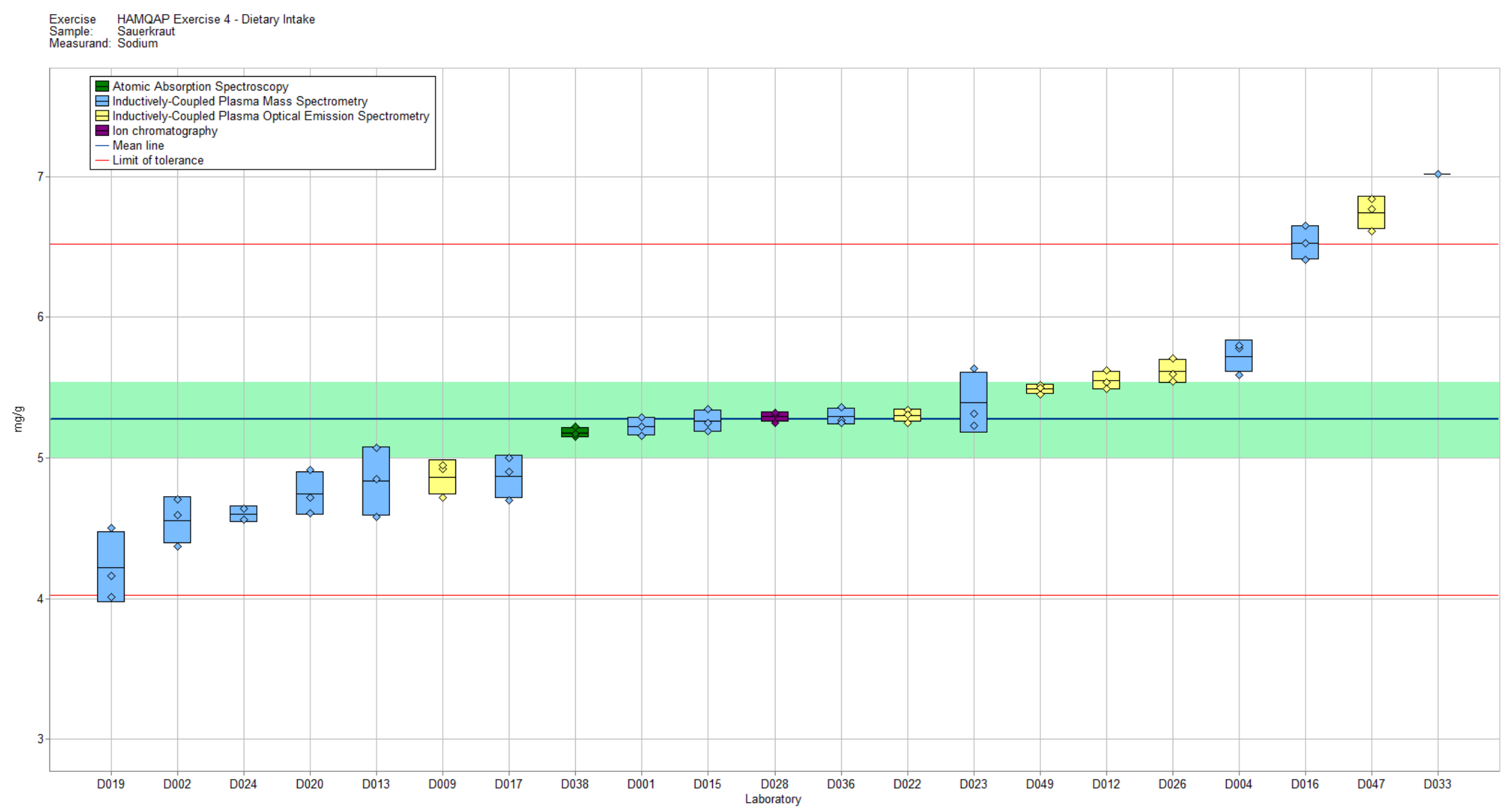

Figure 1-9. Sodium in Sauerkraut (data summary view - analytical method). In this view, individual laboratory data are plotted (diamonds) with the individual laboratory standard deviation (rectangle). The color of the data point represents the analytical method employed. The solid blue line represents the consensus mean, and the green shaded region represents the $95 \%$ confidence interval for the consensus mean. The red solid lines represent the consensus range of tolerance, calculated as the values above and below the consensus mean that result in an acceptable $Z_{\text {comm }}^{\prime}$ score, $\left|Z_{\text {comm }}^{\prime}\right| \leq 2$. A NIST value has not been determined in this material. 


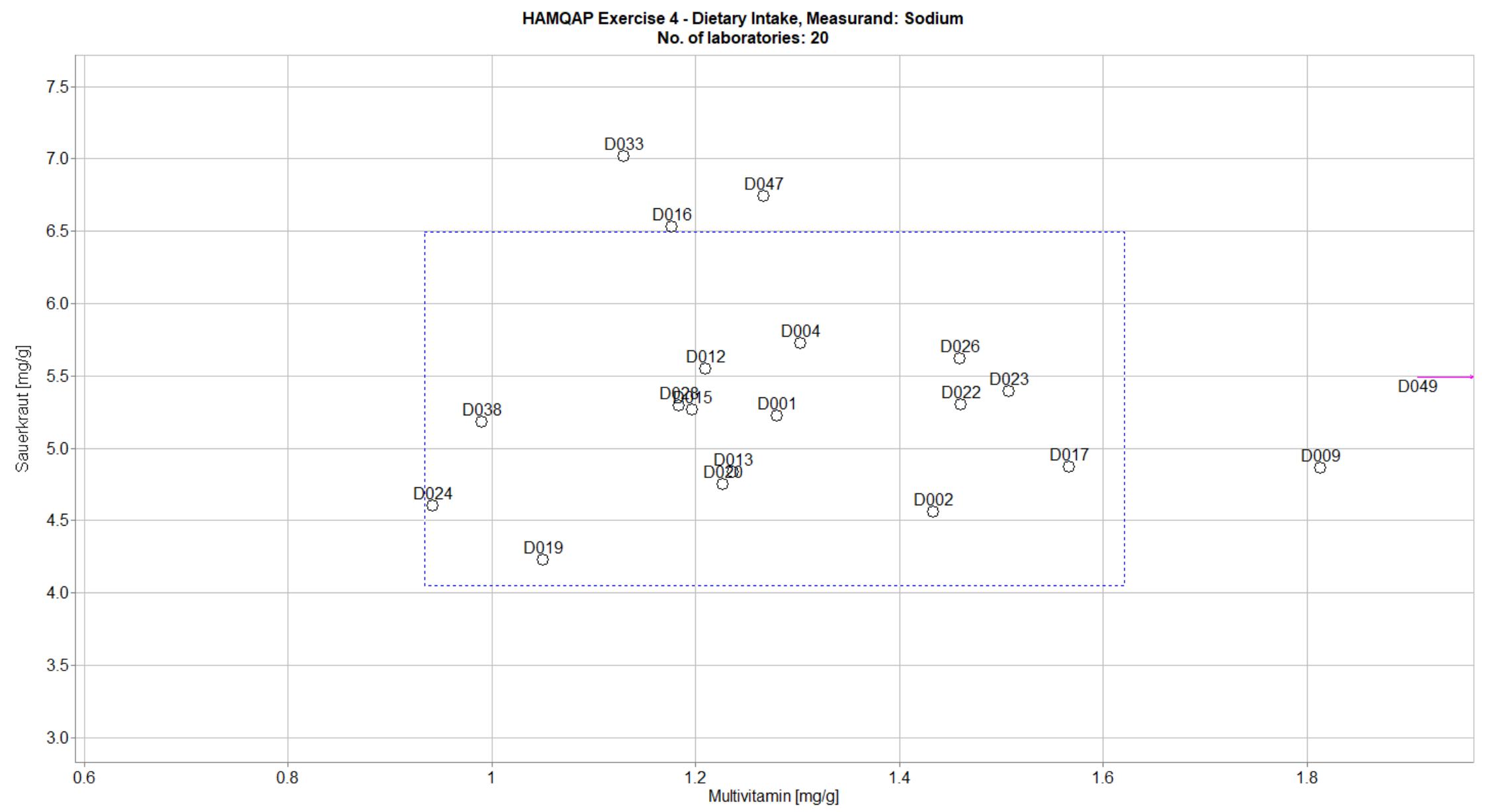

Figure 1-10. Laboratory means for sodium in Multivitamin and Sauerkraut (sample/sample comparison view). In this view, the individual laboratory mean for one sample (multivitamin) is compared to the individual laboratory mean for a second sample (sauerkraut). The dotted blue box represents the consensus range of tolerance for multivitamin (x-axis) and sauerkraut (y-axis), calculated as the values above and below the consensus means that result in an acceptable $Z_{\text {comm }}^{\prime}$ score, $\left|Z_{\text {comm }}^{\prime}\right| \leq 2$. 
Table 1-4. Data summary table for potassium in multivitamin and sauerkraut. Data points highlighted in red have been flagged as potential outliers (e.g., Grubb and/or Cochran) by the NIST software package.

\begin{tabular}{|c|c|c|c|c|c|c|c|c|c|c|c|}
\hline & \multirow[b]{3}{*}{ Lab } & \multicolumn{10}{|c|}{ Potassium } \\
\hline & & \multicolumn{5}{|c|}{ Multivitamin (mg/g) } & \multicolumn{5}{|c|}{ Sauerkraut (mg/g) } \\
\hline & & $\mathbf{A}$ & B & $\mathbf{C}$ & Avg & SD & $\mathbf{A}$ & B & $\mathbf{C}$ & Avg & SD \\
\hline \multirow{37}{*}{ 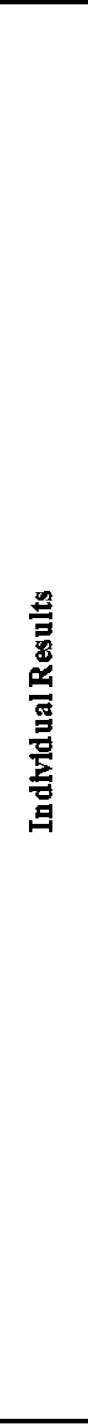 } & Target & & & & 48.0 & 4.0 & & & & & \\
\hline & D001 & 52 & 55.7 & 55.3 & 54.3 & 2.0 & 1.95 & 1.94 & 1.91 & 1.933 & 0.021 \\
\hline & $\mathrm{D} 002$ & 50.248 & 48.122 & 47.051 & 48.5 & 1.6 & 1.8581 & 1.823 & 1.7779 & 1.820 & 0.040 \\
\hline & D003 & & & & & & & & & & \\
\hline & D004 & 48.67 & 52.61 & 51.65 & 51.0 & 2.1 & 2.24 & 1.96 & 2.3 & 2.167 & 0.181 \\
\hline & D005 & 54.46 & 61.95 & 58.82 & 58.4 & 3.8 & & & & & \\
\hline & D006 & 52.13 & 53.24 & 53.26 & 52.9 & 0.6 & & & & & \\
\hline & D007 & & & & & & & & & & \\
\hline & D009 & 107.2 & 109 & 114.7 & 110.3 & 3.9 & 1.82 & 1.93 & 1.81 & 1.853 & 0.067 \\
\hline & D010 & 44.5 & & 43.8 & 44.2 & 0.5 & & & & & \\
\hline & D012 & 53.4 & 58.3 & 54.4 & 55.4 & 2.6 & 1.89 & 1.87 & 1.87 & 1.877 & 0.012 \\
\hline & D013 & 50.8 & 44.8 & 47.2 & 47.6 & 3.0 & 1.79 & 1.89 & 1.96 & 1.880 & 0.085 \\
\hline & D015 & 50.9 & 51.9 & 45.2 & 49.3 & 3.6 & 2 & 2 & 2 & 2.000 & 0.000 \\
\hline & D016 & 50.42 & 48.2 & 45.96 & 48.2 & 2.2 & 1.81 & 1.9 & 1.86 & 1.857 & 0.045 \\
\hline & D017 & 47 & 48 & 42 & 45.7 & 3.2 & 1.7 & 1.7 & 1.6 & 1.667 & 0.058 \\
\hline & D018 & & & & & & & & & & \\
\hline & D019 & 46.95 & 44.09 & 45.59 & 45.5 & 1.4 & 1.84 & 1.72 & 1.63 & 1.730 & 0.105 \\
\hline & D020 & 49.37 & 54.33 & 52.87 & 52.2 & 2.5 & 1.743 & 1.671 & 1.687 & 1.700 & 0.038 \\
\hline & D021 & 52.9 & 52.1 & 52.6 & 52.5 & 0.4 & & & & & \\
\hline & D022 & 57.5 & 58.3 & 55.2 & 57.0 & 1.6 & 2.19 & 2.19 & 2.2 & 2.193 & 0.006 \\
\hline & D023 & 53.295 & 54.214 & 53.805 & 53.8 & 0.5 & 1.8312 & 2.0184 & 1.8568 & 1.902 & 0.102 \\
\hline & D024 & 47.34 & 49.91 & 45.02 & 47.4 & 2.4 & 1.67 & 1.69 & & 1.680 & 0.014 \\
\hline & D026 & 48.285 & 48.25 & 48.863 & 48.5 & 0.3 & 1.826 & 1.804 & 1.793 & 1.808 & 0.017 \\
\hline & D027 & & & & & & & & & & \\
\hline & D028 & 50.3 & 48.8 & 48.3 & 49.1 & 1.0 & 1.96 & 1.94 & 1.99 & 1.963 & 0.025 \\
\hline & D031 & 53.96 & 51.01 & 50.66 & 51.9 & 1.8 & & & & & \\
\hline & D032 & & & & & & & & & & \\
\hline & D033 & 49.55 & 50.43 & 55.97 & 52.0 & 3.5 & 1.7 & & & 1.700 & \\
\hline & D034 & & & & & & & & & & \\
\hline & D035 & 45.92 & 47.79 & 47.79 & 47.2 & 1.1 & & & & & \\
\hline & D036 & 57.62 & 58.59 & 60.01 & 58.7 & 1.2 & 1.78 & 1.83 & 1.78 & 1.797 & 0.029 \\
\hline & D038 & 38.42 & 41.46 & 41.1 & 40.3 & 1.7 & 1.85 & 1.85 & 1.83 & 1.843 & 0.012 \\
\hline & D045 & & & & & & & & & & \\
\hline & D046 & & & & & & & & & & \\
\hline & D047 & 46.6 & 42.4 & 50.4 & 46.5 & 4.0 & 1.941 & 1.824 & 1.824 & 1.863 & 0.068 \\
\hline & D049 & 258 & 49.7 & 51.6 & 119.8 & 119.7 & 1.89 & 1.87 & 1.9 & 1.887 & 0.015 \\
\hline & D050 & 49.624 & 46.396 & 49.285 & 48.4 & 1.8 & & & & & \\
\hline 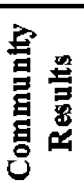 & & $\begin{array}{l}\text { Consensu } \\
\text { Consensu } \\
\text { Maximun } \\
\text { Minimum } \\
\text { N }\end{array}$ & $\begin{array}{l}\text { Mean } \\
\text { Standard }\end{array}$ & Peviation & $\begin{array}{c}50.3 \\
1.0 \\
119.8 \\
40.3 \\
28\end{array}$ & & $\begin{array}{l}\text { Consens } \\
\text { Consens } \\
\text { Maximu } \\
\text { Minimur } \\
\text { N }\end{array}$ & $\begin{array}{l}\text { Mean } \\
\text { Standard }\end{array}$ & eviation & $\begin{array}{c}1.850 \\
0.028 \\
2.193 \\
1.667 \\
20\end{array}$ & \\
\hline
\end{tabular}




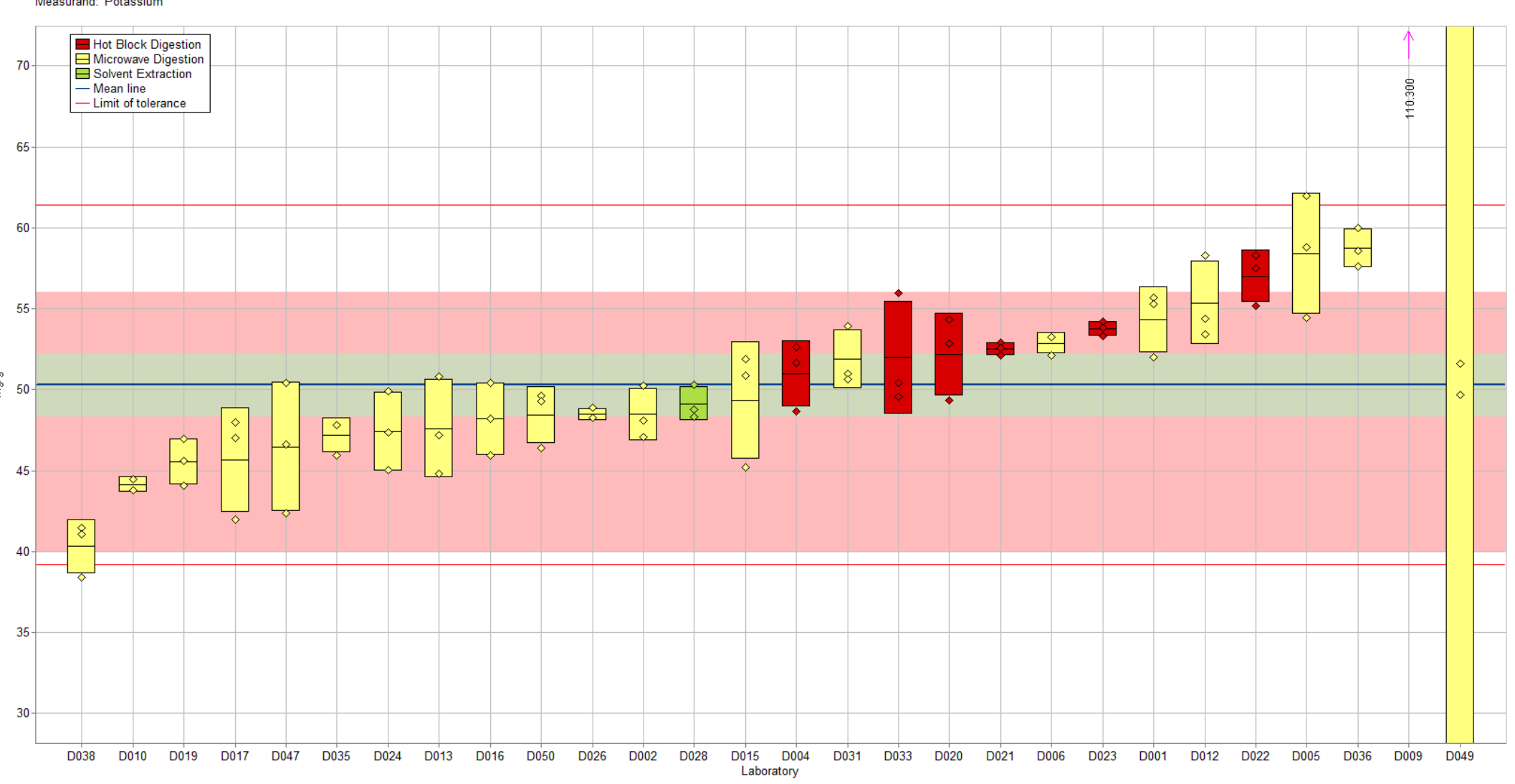

Figure 1-11. Potassium in Multivitamin (data summary view - sample preparation method). In this view, individual laboratory data are plotted (diamonds) with the individual laboratory standard deviation (rectangle). The color of the data point represents the sample preparation method employed. The solid blue line represents the consensus mean, and the green shaded region represents the $95 \%$ confidence interval for the consensus mean. The red solid lines represent the consensus range of tolerance, calculated as the values above and below the consensus mean that result in an acceptable $Z_{\text {comm }}^{\prime}$ score, $\left|Z_{\text {comm }}^{\prime}\right| \leq 2$. The red shaded region represents the NIST range of tolerance, which encompasses the target value bounded by twice its uncertainty $\left(U_{\mathrm{NIST}}\right)$ and represents the range that results in an acceptable $Z_{\text {NIST }}$ score, $\left|Z_{\text {NIST }}\right| \leq 2$. 


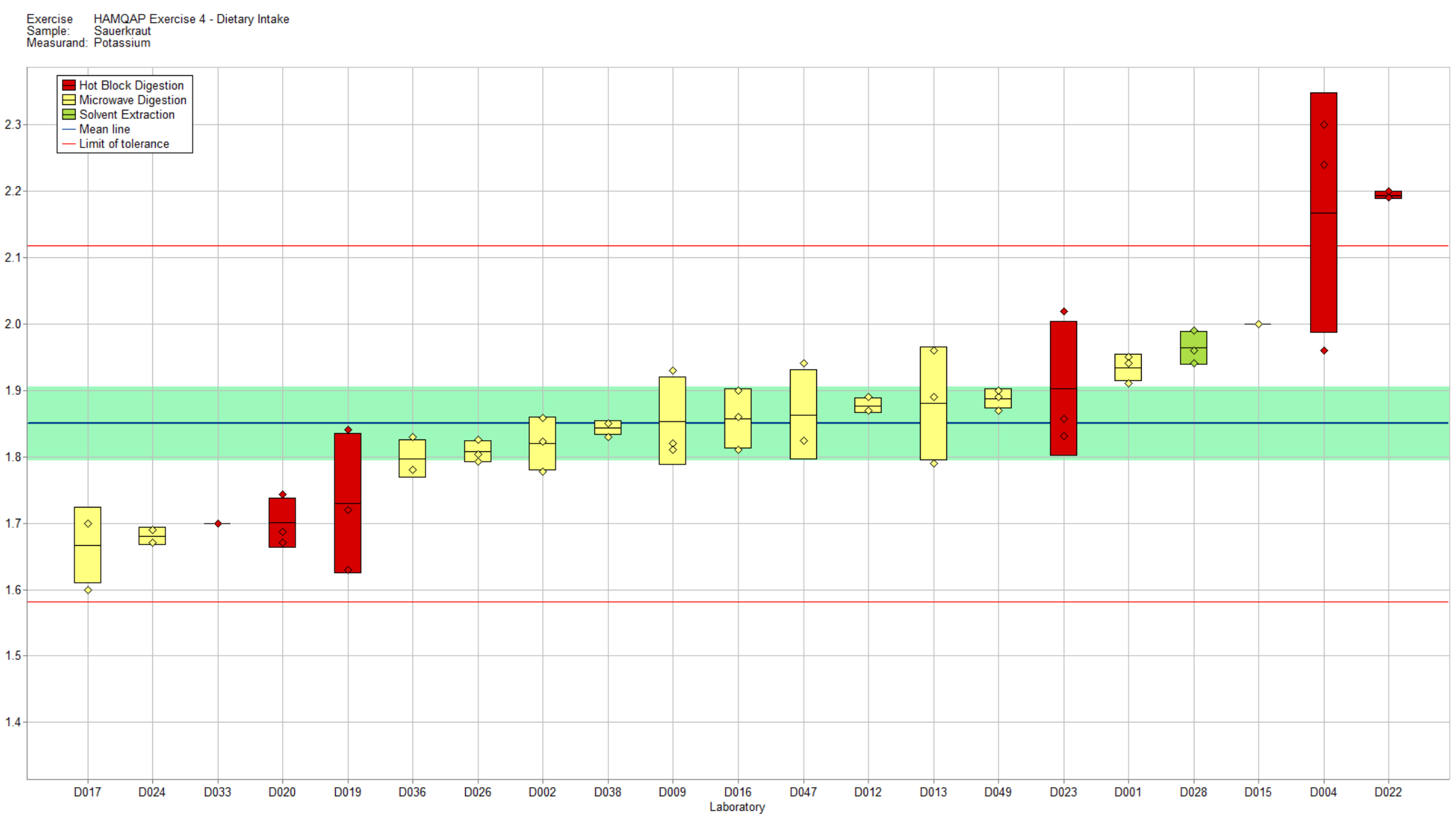

Figure 1-12. Potassium in Sauerkraut (data summary view - sample preparation method). In this view, individual laboratory data are plotted (diamonds) with the individual laboratory standard deviation (rectangle). The color of the data point represents the sample preparation method employed. The solid blue line represents the consensus mean, and the green shaded region represents the $95 \%$ confidence interval for the consensus mean. The red solid lines represent the consensus range of tolerance, calculated as the values above and below the consensus mean that result in an acceptable $Z_{\text {comm }}^{\prime}$ score, $\left|Z_{\text {comm }}^{\prime}\right| \leq 2$. A NIST value has not been determined in this material. 


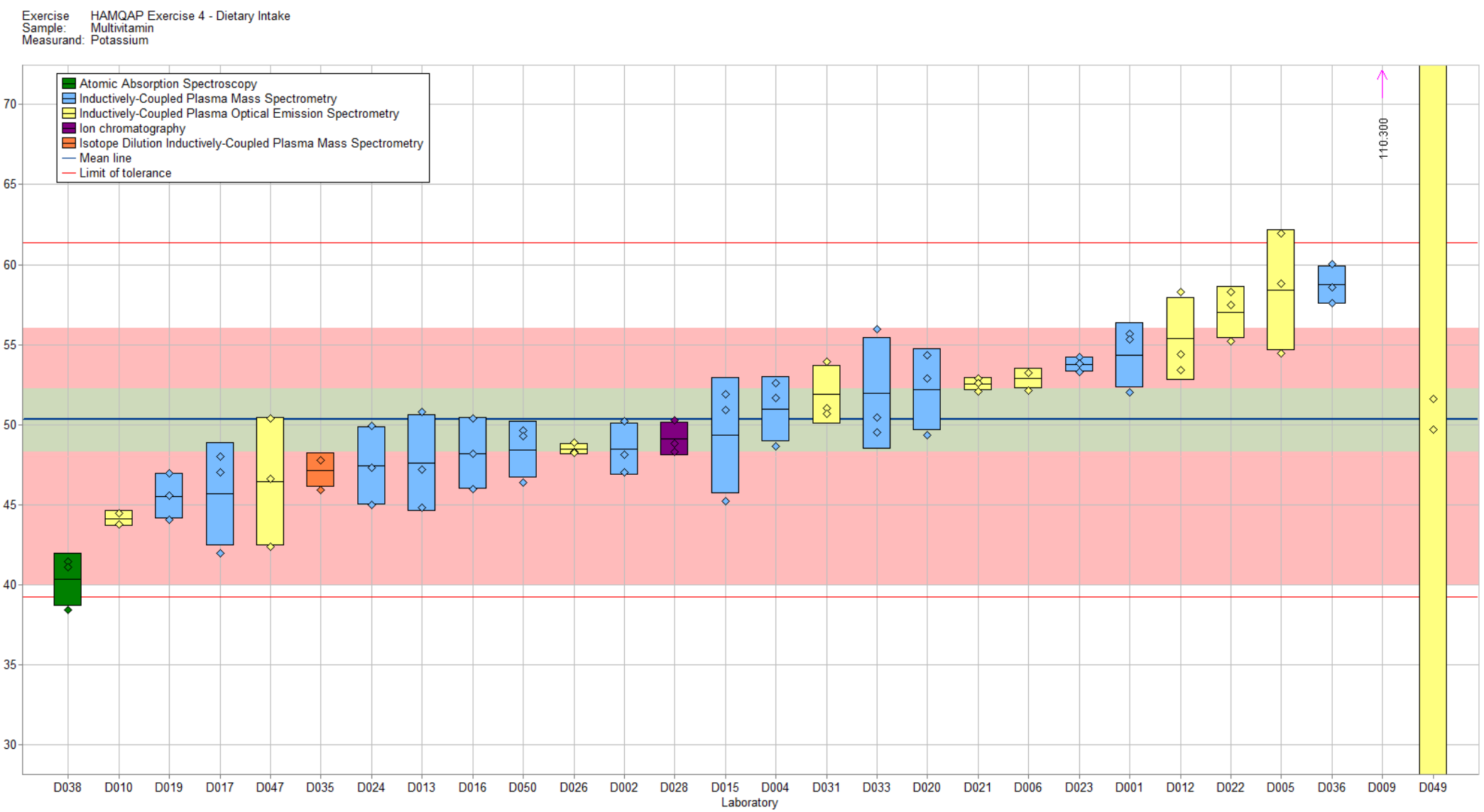

Figure 1-13. Potassium in Multivitamin (data summary view - analytical method). In this view, individual laboratory data are plotted (diamonds) with the individual laboratory standard deviation (rectangle). The color of the data point represents the analytical method employed. The solid blue line represents the consensus mean, and the green shaded region represents the $95 \%$ confidence interval for the consensus mean. The red solid lines represent the consensus range of tolerance, calculated as the values above and below the consensus mean that result in an acceptable $Z_{\text {comm }}^{\prime}$ score, $\left|Z_{\text {comm }}^{\prime}\right| \leq 2$. The red shaded region represents the NIST range of tolerance, which encompasses the target value bounded by twice its uncertainty ( $\left.U_{\text {NIST }}\right)$ and represents the range that results in an acceptable $Z_{\text {NIST }}$ score, $\left|Z_{\mathrm{NIST}}\right| \leq 2$. 
Exercise HAMQAP Exercise 4 - Dietary Intake
Sample:

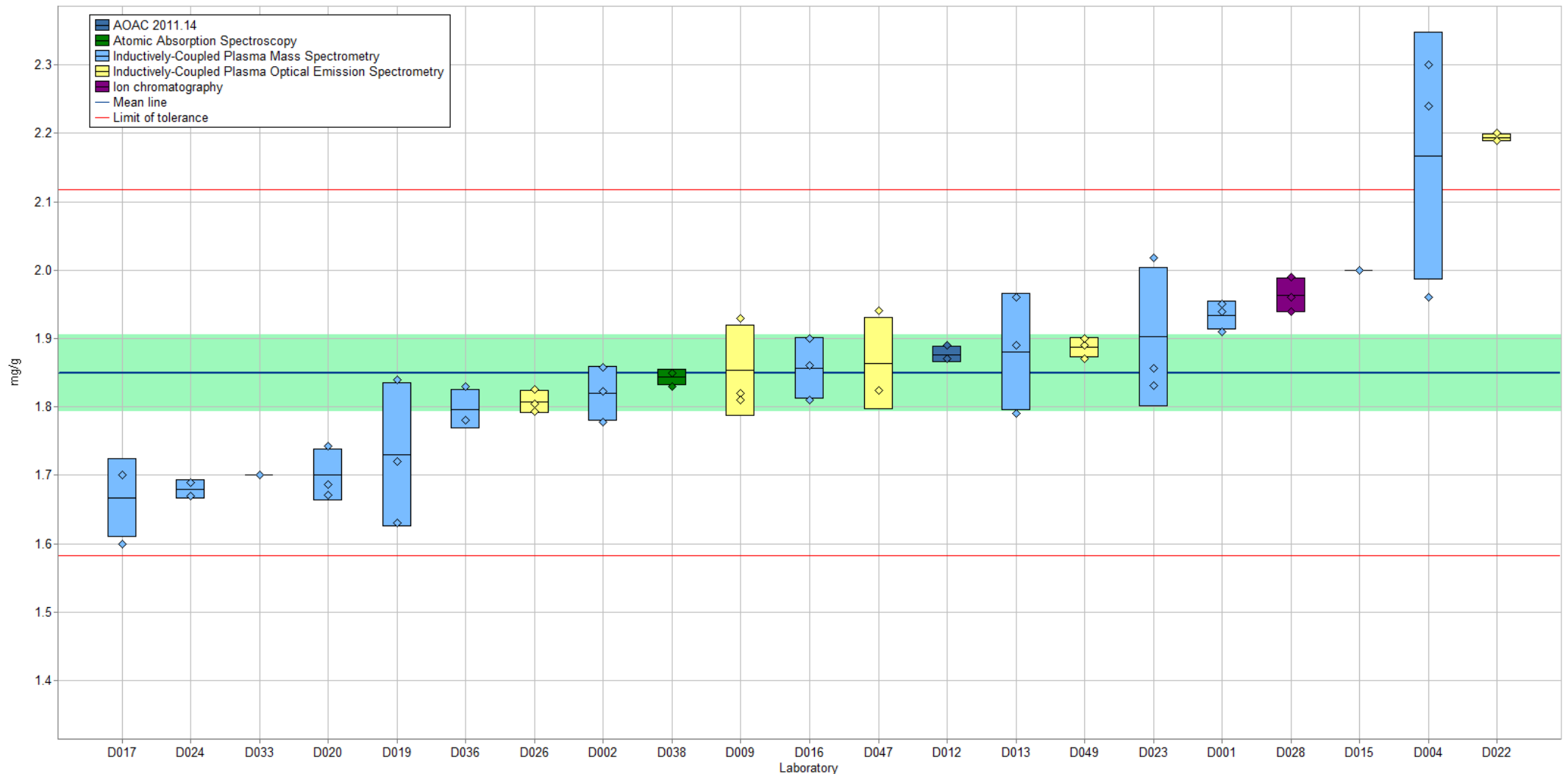

Figure 1-14. Potassium in Sauerkraut (data summary view - analytical method). In this view, individual laboratory data are plotted (diamonds) with the individual laboratory standard deviation (rectangle). The color of the data point represents the analytical method employed. The solid blue line represents the consensus mean, and the green shaded region represents the $95 \%$ confidence interval for the consensus mean. The red solid lines represent the consensus range of tolerance, calculated as the values above and below the consensus mean that result in an acceptable $Z_{\text {comm }}^{\prime}$ score, $\left|Z_{\text {comm }}^{\prime}\right| \leq 2$. A NIST value has not been determined in this material. 


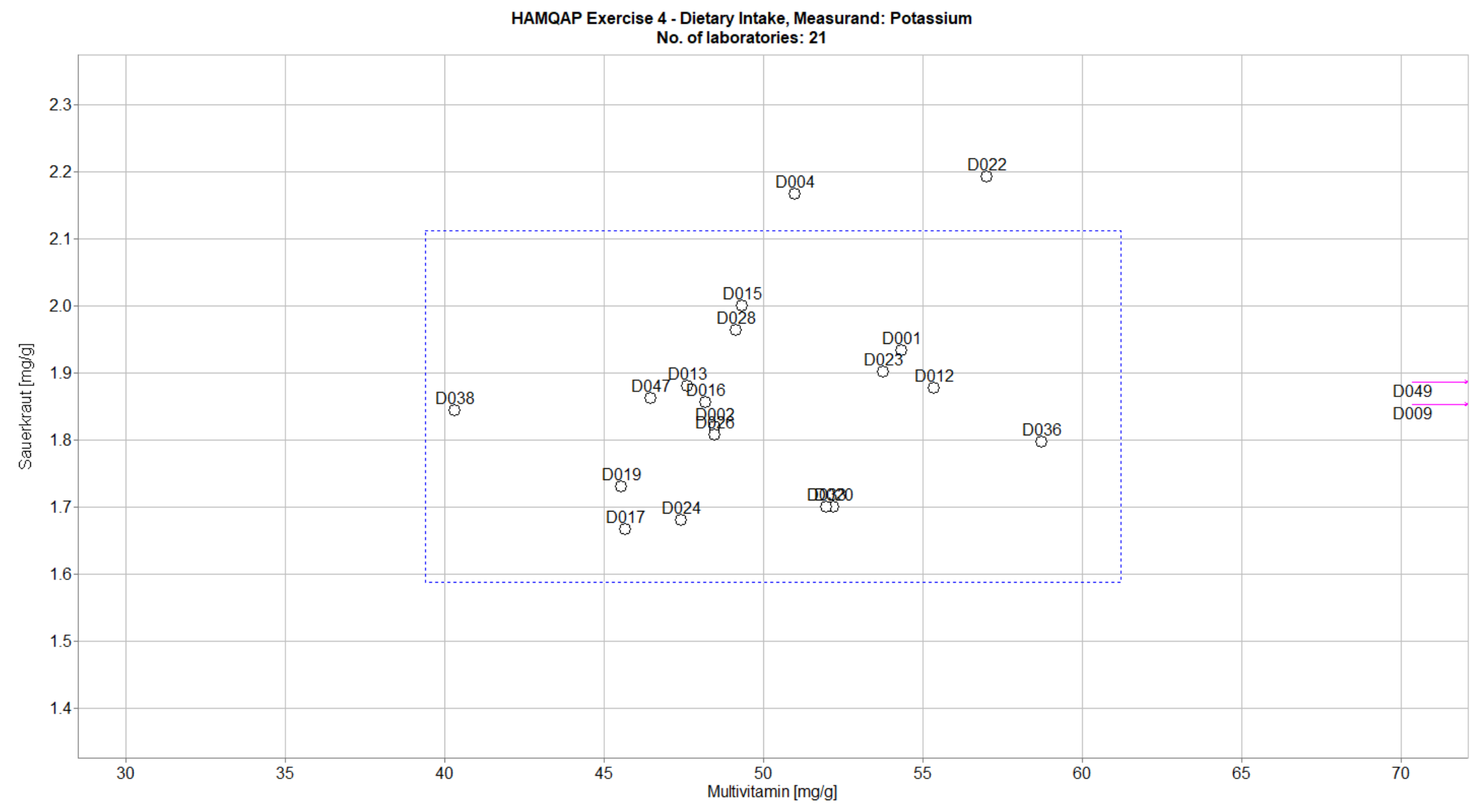

Figure 1-15. Laboratory means for potassium in Multivitamin and Sauerkraut (sample/sample comparison view). In this view, the individual laboratory mean for one sample (multivitamin) is compared to the individual laboratory mean for a second sample (sauerkraut). The dotted blue box represents the consensus range of tolerance for multivitamin (x-axis) and sauerkraut (y-axis), calculated as the values above and below the consensus means that result in an acceptable $Z_{\text {comm }}^{\prime}$ score, $\left|Z_{\text {comm }}^{\prime}\right| \leq 2$. 


\section{SECTION 2: TOXIC ELEMENTS (Cadmium, Lead)}

\section{Study Overview}

In this study, participants were provided with samples of SRM 2384 Baking Chocolate and SRM 2387 Peanut Butter for dietary intake. Participants were asked to use in-house analytical methods to determine the mass fractions $(\mathrm{mg} / \mathrm{kg})$ of cadmium $(\mathrm{Cd})$ and lead $(\mathrm{Pb})$ in each food matrix. Lead and cadmium are toxic elements that may be released into the environment through anthropogenic activities including mining, incineration of municipal waste, manufacturing and smelting, disposal of sewage, lead paint deposits, and application of fertilizers or pesticides. Potential uptake of toxic elements from the soil may lead to contamination of plant-based foods and dietary supplements and thus lead to negative health outcomes for consumers. ${ }^{7,8}$ In the United States, cGMPs require food manufacturers to establish limits on contaminants, therefore laboratories must establish scientifically valid methods for the determination of toxic elements to demonstrate the products meet the specifications in the U.S. FDA Code of Federal Regulations (21 CFR 111.70(b)(3)).

Dietary Intake Sample Information

Baking Chocolate. Participants were provided with one piece of chocolate weighing approximately $20 \mathrm{~g}$. Participants were asked to store the material under refrigeration between $2{ }^{\circ} \mathrm{C}$ to $8{ }^{\circ} \mathrm{C}$ until use, and to prepare three samples and to report three values from the single piece of chocolate provided. Before use, participants were instructed to melt or grate the bar and to use a sample size of at least $0.5 \mathrm{~g}$. Approximate analyte levels were not reported to participants prior to the study. Certified values were assigned for $\mathrm{Cd}$ and $\mathrm{Pb}$ using results from NIST by ID ICP-MS. The NIST-determined values and uncertainties are provided in the table below on an as-received basis.

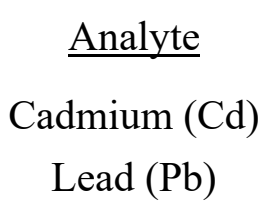

Peanut Butter. Participants were provided with one jar containing approximately $170 \mathrm{~g}$ of peanut butter. Participants were asked to store the material at $-20{ }^{\circ} \mathrm{C}$ in the original unopened jar, and to prepare three samples and report three values from the jar provided. Before use, participants were instructed to thoroughly mix the contents of the jar and to use a sample size of at least $0.5 \mathrm{~g}$. Approximate analyte levels were not reported to participants prior to the study. Target values for $\mathrm{Cd}$ and $\mathrm{Pb}$ in the peanut butter were assigned using results from NIST by ICP-MS. The NISTdetermined values and uncertainties are provided in the table below on an as-received basis.

\footnotetext{
${ }^{7}$ Cadmium Factsheet. National Biomonitoring Program, Centers for Disease Control and Prevention. https://www.cdc.gov/biomonitoring/Cadmium_FactSheet.html (accessed February 2020).

${ }^{8}$ Lead Factsheet. National Biomonitoring Program, Centers for Disease Control and Prevention. https://www.cdc.gov/biomonitoring/Lead_factsheet.html (accessed February 2020).
} 


\section{Analyte \\ Cadmium $(\mathrm{Cd})$ \\ Lead $(\mathrm{Pb})$}

NIST-Determined Mass Fractions in

\begin{tabular}{lll}
\multicolumn{2}{c}{ Peanut Butter $(\mathrm{mg} / \mathrm{kg})$} \\
\hline $0.05589 \pm 0.00086$ \\
$0.0023 \pm 0.0013$
\end{tabular}

Dietary Intake Study Results

- Thirty-one laboratories enrolled in this exercise and received samples to measure $\mathrm{Cd}$ and/or $\mathrm{Pb}$. The table below lists the participation statistics for each analyte. Some of the reported values were non-quantitative (zero or below LOQ) but are included in the participation and reporting statistics.

\begin{tabular}{|c|c|c|c|}
\hline \multirow[b]{2}{*}{ Analyte } & $\underline{\text { Number of }}$ & \multicolumn{2}{|c|}{$\frac{\text { Number of Laboratories Reporting Results }}{\text { (Percent Participation) }}$} \\
\hline & $\underline{\text { Requesting Samples }}$ & Peanut Butter & Baking Chocolate \\
\hline $\mathrm{Cd}$ & 31 & $22(71 \%)$ & $21(68 \%)$ \\
\hline $\mathrm{Pb}$ & 30 & $19(63 \%)$ & $21(70 \%)$ \\
\hline
\end{tabular}

- The consensus range was within the target range or overlapped the target range for both analytes in both materials.

- The between-laboratory variabilities for $\mathrm{Cd}$ each sample and for $\mathrm{Pb}$ in the baking chocolate were excellent ( $2 \%$ to $4 \%$ ). The between-laboratory variability for $\mathrm{Pb}$ in the peanut butter was greater at $19 \%$ (see table below).

\begin{tabular}{ccc} 
& \multicolumn{2}{c}{ Between-Laboratory Variability (\% RSD) } \\
Analyte & Peanut Butter & Baking Chocolate \\
\cline { 2 - 2 } & $2 \%$ & $2 \%$ \\
$\mathrm{~Pb}$ & $19 \%$ & $4 \%$
\end{tabular}

- Most laboratories reported using microwave digestion for determination of toxic elements (see table below).

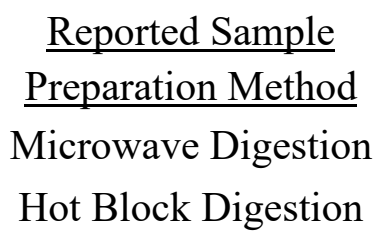

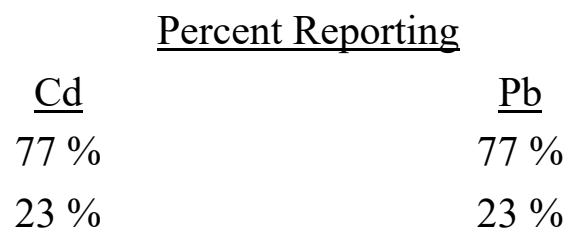

- Most laboratories reported using ICP-MS for determination of toxic elements (see table below). One laboratory reported using AAS to determine $\mathrm{Cd}$ in both chocolate and peanut butter, and one laboratory reported using ID ICP-MS to determine $\mathrm{Pb}$ in both chocolate and peanut butter. Two additional laboratories also reported using ICP-OES for the determination of Cd in peanut butter. 


\section{Dietary Intake Technical Recommendations}

The following observations and recommendations are based on results obtained from the participants in this study.

- No significant bias was observed between the results obtained by different instrumental techniques in either sample or for either analyte.

- The mean concentrations reported by laboratories using microwave digestion was higher than those using hot block digestion, but too few laboratories reported using hot block digestion to determine conclusively identify the source of bias.

- Between-laboratory variability was very low for $\mathrm{Cd}$ in both samples and for $\mathrm{Pb}$ in chocolate. The between-laboratory variability was much higher for $\mathrm{Pb}$ in peanut butter; laboratories may have had difficulty determining the very low levels of $\mathrm{Pb}$, which was ten times lower in the peanut butter than in the chocolate.

- Both chocolate and peanut butter are high in fat, increasing the difficulty of sample preparation compared to lower fat materials. Established quality control materials (SRMs, CRMs, RMs, and in-house materials) and accepted methods of analysis can verify that sample preparation methods are properly implemented before analyzing unknowns.

- The low levels of $\mathrm{Cd}$ and $\mathrm{Pb}$ in these samples may have been challenging for participants.

- Limiting the number of sample dilutions may improve the ability to detect $\mathrm{Cd}$ and $\mathrm{Pb}$ at low levels in these materials, although matrix effects may become more significant. A matrix-matched calibration curve may reduce some of the matrix interferences.

- Determination of LOQ and MDL is important when analyte concentrations are low. Analysis of an appropriate number of procedural blanks can be critical in the determination of LOQ and MDL or when trying to reduce sample-to-sample variability. Analysis of many blanks can provide information about whether the variability is arising from the sample preparation procedure. The suggested minimum number of blanks to prepare is equal to the number of samples being prepared.

- For cadmium, approximately half of the laboratories reported data that were within the $95 \%$ confidence interval for the consensus mean for both materials (Figures 2-1 through 2-4).

- Figure 2-5 shows few laboratories were able to measure both samples accurately and only a few reported results were within the NIST target range for both samples. Those laboratories that reported low results may have had problems with sample preparation.

- Hot block may not be the best sample preparation choice for measuring $\mathrm{Cd}$ in these sample matrices (Figure 2-2 and Figure 2-4). Microwave approaches will reach higher temperatures and provide a more complete digestion.

- Spectral interferences, occurring in the form of isobaric interferences where the interference has the same nominal mass as the isotope of interest, or non-spectral interferences, signal suppression or enhancement stemming from the major matrix elements in the matrix, can make $\mathrm{Cd}$ difficult to measure accurately by ICP-MS.

- High concentrations of elements such as Mo, $\mathrm{Sn}$, and $\mathrm{Zr}$ are known to cause isobaric interferences in the analysis of Cd by ICP-MS.

- Performing screens or semi-quantitative scans of the sample before quantitative analysis will indicate any potential interferences in the sample. Collision cell technology can be used to minimize such molecular interferences.

- Many laboratories reported results within the NIST target range for $\mathrm{Pb}$ in both samples (Figure 2-10). 
- For $\mathrm{Pb}$ in baking chocolate, Figure 2-6 and 2-7 show that just over a third of the laboratories reported data within the $95 \%$ confidence interval of the consensus mean.

- The $\mathrm{Pb}$ in the peanut butter was very low, and for approximately one third of the laboratories the concentration was below their LOQ (Figures 2-8 and 2-9).

- Lead is easily digested and volatile loss of $\mathrm{Pb}$ is not a concern; however, use of $\mathrm{HCl}$ in the digestion may result in insoluble $\mathrm{PbCl}_{2}$ precipitate, so digestion with $\mathrm{HNO}_{3}$ is recommended.

- Although time consuming, preconcentration and separation techniques may increase the concentration of lead in solutions prior to analysis and allow better precision and accuracy to be achieved for samples with lower concentrations.

- Calibration curves must be linear and include the lowest and highest values expected to be measured in the sample solutions for best results.

- The use of appropriate calibration materials and quality assurance samples to establish that a method is in control and being performed correctly may reduce the likelihood of outlying data. Quality assurance samples can be commercially available reference materials (CRMs, SRMs, or RMs) or materials prepared in-house. 
Table 2-1. Individualized data summary table (NIST) for toxic elements in baking chocolate and peanut butter.

\section{National Institute of Standards \& Technology}

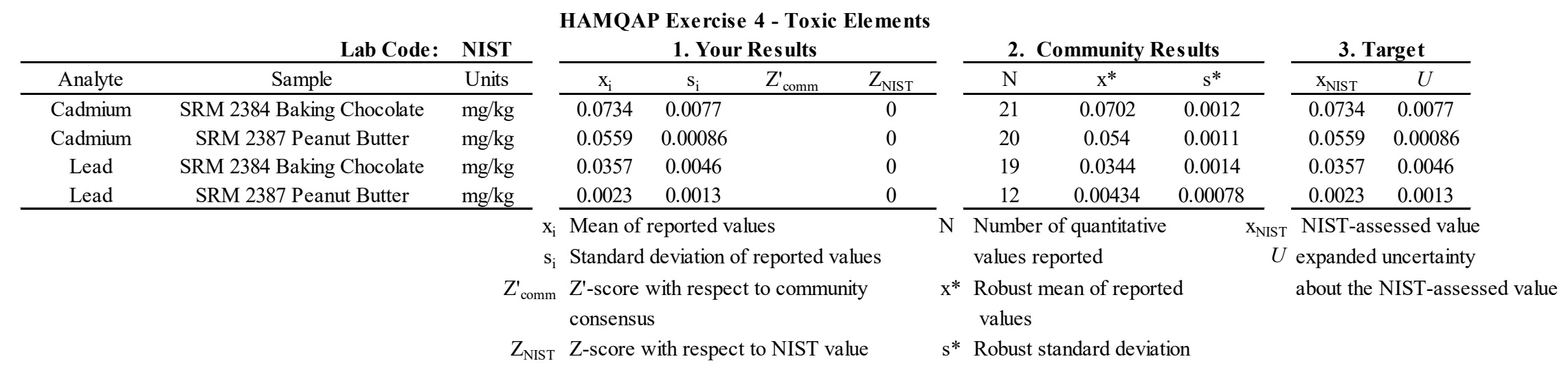


Table 2-2. Data summary table for cadmium in baking chocolate and peanut butter. Data points highlighted in red have been flagged as potential outliers (e.g., Grubb and/or Cochran) by the NIST software package.

\begin{tabular}{|c|c|c|c|c|c|c|c|c|c|c|c|}
\hline & \multirow[b]{3}{*}{ Lab } & \multicolumn{10}{|c|}{ Cadmium } \\
\hline & & \multicolumn{5}{|c|}{ SRM 2384 Baking Chocolate (mg/kg) } & \multicolumn{5}{|c|}{ SRM 2387 Peanut Butter (mg/kg) } \\
\hline & & $\mathbf{A}$ & $\mathbf{B}$ & $\mathbf{C}$ & Avg & SD & $\bar{A}$ & $\mathbf{B}$ & $\mathbf{C}$ & Avg & SD \\
\hline \multirow{32}{*}{ 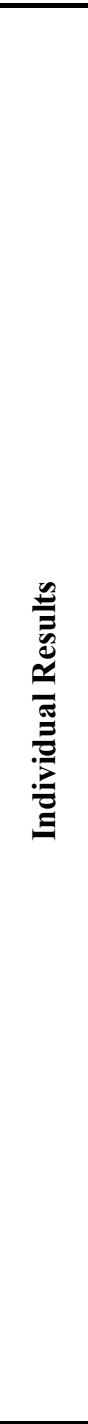 } & Target & & & & 0.0734 & 0.0077 & & & & 0.0559 & 0.00086 \\
\hline & D001 & & & & & & & & & & \\
\hline & D002 & 0.069 & 0.0678 & 0.0599 & 0.0656 & 0.0049 & $<0.010$ & $<0.010$ & $<0.010$ & & \\
\hline & D004 & 0.08 & 0.07 & 0.08 & 0.0767 & 0.0058 & 0.06 & 0.06 & 0.06 & 0.0600 & 0.0000 \\
\hline & D005 & & & & & & & & & & \\
\hline & D006 & & & & & & 0.055 & 0.058 & 0.055 & 0.0560 & 0.0017 \\
\hline & D007 & & & & & & & & & & \\
\hline & D009 & 0.066 & 0.067 & 0.066 & 0.0663 & 0.0006 & 0.052 & 0.054 & 0.054 & 0.0533 & 0.0012 \\
\hline & D010 & & & & & & & & & & \\
\hline & D012 & 0.0749 & 0.075 & 0.0771 & 0.0757 & 0.0012 & 0.057 & 0.0584 & 0.0608 & 0.0587 & 0.0019 \\
\hline & D013 & 0.0723 & 0.0721 & 0.0659 & 0.0701 & 0.0036 & 0.0498 & 0.0474 & 0.0513 & 0.0495 & 0.0020 \\
\hline & D015 & 0.068 & 0.067 & 0.065 & 0.0667 & 0.0015 & 0.056 & 0.058 & 0.059 & 0.0577 & 0.0015 \\
\hline & D016 & & & & & & & & & & \\
\hline & D017 & 0.07 & 0.07 & 0.07 & 0.0700 & 0.0000 & 0.04 & 0.05 & 0.06 & 0.0500 & 0.0100 \\
\hline & D019 & 0.00 & 0.06 & 0.06 & 0.0403 & 0.0341 & 0.05 & 0.0003 & 0.001 & 0.0171 & 0.0285 \\
\hline & D020 & 0.0695 & 0.0578 & 0.0619 & 0.0631 & 0.0059 & 0.0542 & 0.0393 & 0.0478 & 0.0471 & 0.0075 \\
\hline & D021 & 0.0788 & 0.0726 & 0.0698 & 0.0737 & 0.0046 & 0.0579 & 0.0553 & 0.0552 & 0.0561 & 0.0015 \\
\hline & D022 & 0.072 & 0.071 & 0.071 & 0.0713 & 0.0006 & 0.042 & 0.044 & 0.044 & 0.0433 & 0.0012 \\
\hline & D023 & 0.0614 & 0.0615 & 0.064 & 0.0623 & 0.0015 & 0.052 & 0.052 & 0.05 & 0.0513 & 0.0012 \\
\hline & D024 & 0.075 & 0.073 & & 0.0740 & 0.0014 & 0.05 & 0.051 & & 0.0505 & 0.0007 \\
\hline & D027 & 0.0723 & 0.0676 & 0.0738 & 0.0712 & 0.0032 & 0.0561 & 0.0525 & 0.0611 & 0.0566 & 0.0043 \\
\hline & D030 & 0.0703 & 0.0718 & 0.0752 & 0.0724 & 0.0025 & 0.0607 & 0.0541 & 0.0595 & 0.0581 & 0.0035 \\
\hline & D033 & 0.076 & 0.071 & 0.074 & 0.0737 & 0.0025 & 0.058 & 0.057 & 0.054 & 0.0563 & 0.0021 \\
\hline & D034 & & & & & & & & & & \\
\hline & D036 & 0.09 & 0.08 & 0.08 & 0.0833 & 0.0058 & $<0.000$ & $<0.000$ & $<0.000$ & & \\
\hline & D038 & 0.067 & 0.068 & 0.068 & 0.0677 & 0.0006 & 0.057 & 0.057 & 0.052 & 0.0553 & 0.0029 \\
\hline & D041 & & & & & & & & & & \\
\hline & D045 & & & & & & & & & & \\
\hline & D046 & & & & & & & & & & \\
\hline & D047 & 0.0719 & 0.0695 & 0.0701 & 0.0705 & 0.0013 & 0.0561 & 0.0546 & 0.0582 & 0.0563 & 0.0018 \\
\hline & D049 & 0.0666 & 0.0649 & 0.0656 & 0.0657 & 0.0009 & 0.0533 & 0.0523 & 0.052 & 0.0525 & 0.0007 \\
\hline & D050 & 0.07 & 0.068 & 0.069 & 0.0690 & 0.0010 & 0.054 & 0.054 & 0.054 & 0.0540 & 0.0000 \\
\hline 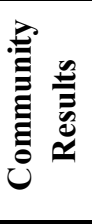 & & $\begin{array}{l}\text { Consens } \\
\text { Consens } \\
\text { Maximun } \\
\text { Minimum } \\
\mathrm{N} \\
\end{array}$ & $\begin{array}{l}\text { Mean } \\
\text { Standard }\end{array}$ & Deviation & $\begin{array}{c}0.0702 \\
0.0013 \\
0.0833 \\
0.0403 \\
21 \\
\end{array}$ & & $\begin{array}{l}\text { Consens } \\
\text { Consens } \\
\text { Maximur } \\
\text { Minimun } \\
\mathrm{N} \\
\end{array}$ & $\begin{array}{l}\text { s Mean } \\
\text { s Standarc }\end{array}$ & Deviation & $\begin{array}{c}0.0540 \\
0.0011 \\
0.0600 \\
0.0171 \\
20 \\
\end{array}$ & \\
\hline
\end{tabular}


Exercise HAMQAP Exercise 4- Dietary Intake
Sample:
Meas

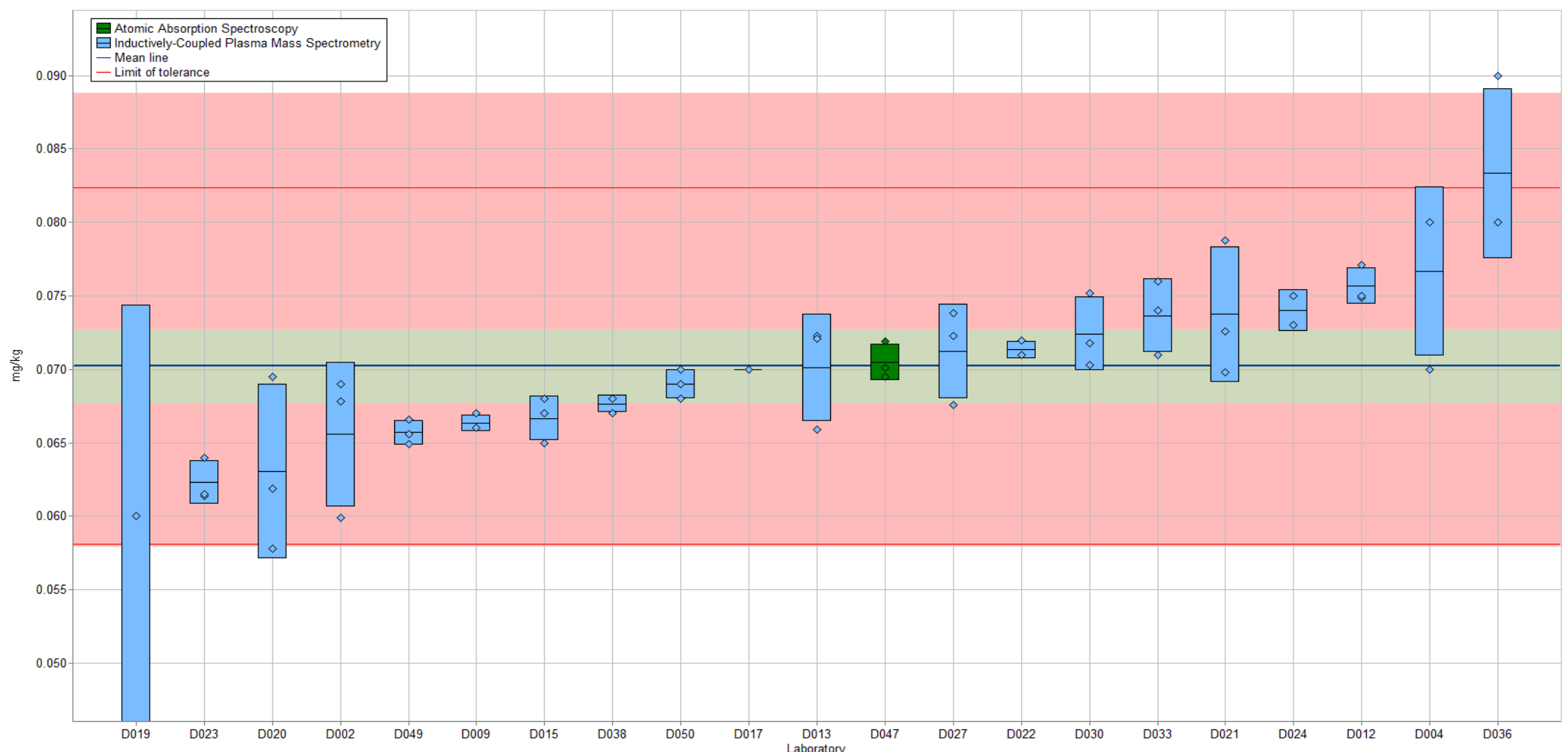

Figure 2-1. Cadmium in SRM 2384 Baking Chocolate (data summary view - analytical method). In this view, individual laboratory data are plotted (diamonds) with the individual laboratory standard deviation (rectangle). The color of the data point represents the analytical method employed. The solid blue line represents the consensus mean, and the green shaded region represents the $95 \%$ confidence interval for the consensus mean. The solid red lines represent the consensus range of tolerance, calculated as the values above and below the consensus mean that result in an acceptable $Z_{\text {comm }}^{\prime}$ score, $\left|Z_{\text {comm }}^{\prime}\right| \leq 2$. The red shaded region represents the NIST range of tolerance, which encompasses the target value bounded by twice its uncertainty $\left(U_{\text {NIST }}\right)$ and represents the range that results in an acceptable $Z_{\text {NIST }}$ score, $\left|Z_{\text {NIST }}\right| \leq 2$. 
Exercise
HAMAAPE Exercise 4 - Dietary Intake
Sample:
Measurand: Cadmium Bakking Chocolate

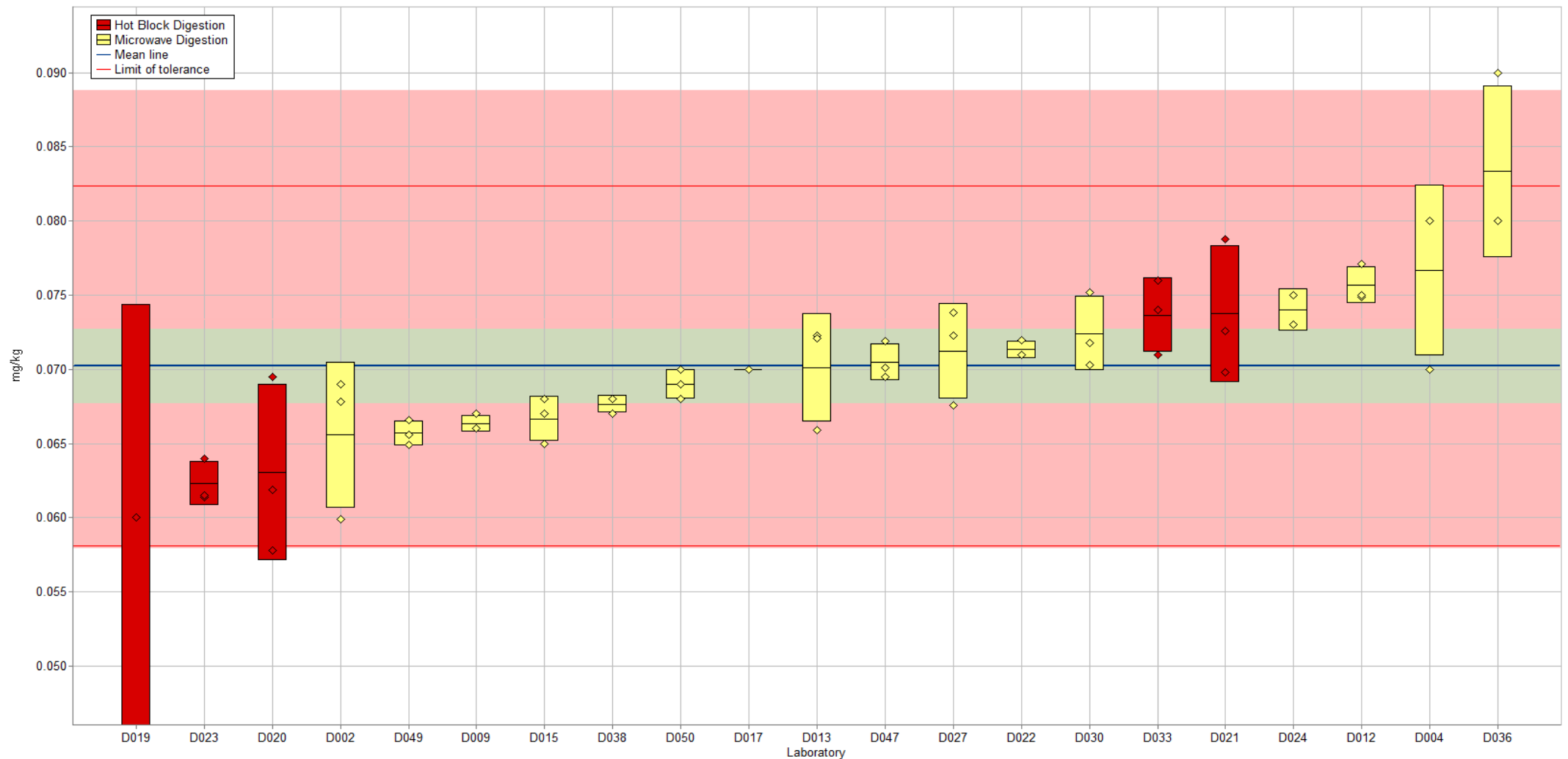

Figure 2-2. Cadmium in SRM 2384 Baking Chocolate (data summary view -sample preparation method). In this view, individual laboratory data are plotted (diamonds) with the individual laboratory standard deviation (rectangle). The color of the data point represents the sample preparation method employed. The solid blue line represents the consensus mean, and the green shaded region represents the $95 \%$ confidence interval for the consensus mean. The solid red lines represent the consensus range of tolerance, calculated as the values above and below the consensus mean that result in an acceptable $Z_{\text {comm }}^{\prime}$ score, $\left|Z_{\text {comm }}^{\prime}\right| \leq 2$, with the lower range set at zero. The red shaded region represents the NIST range of tolerance, which encompasses the target value bounded by twice its uncertainty ( $U_{\text {NIST }}$ ) and represents the range that results in an acceptable $Z_{\text {NIST }}$ score, $\left|Z_{\text {NIST }}\right| \leq 2$. 


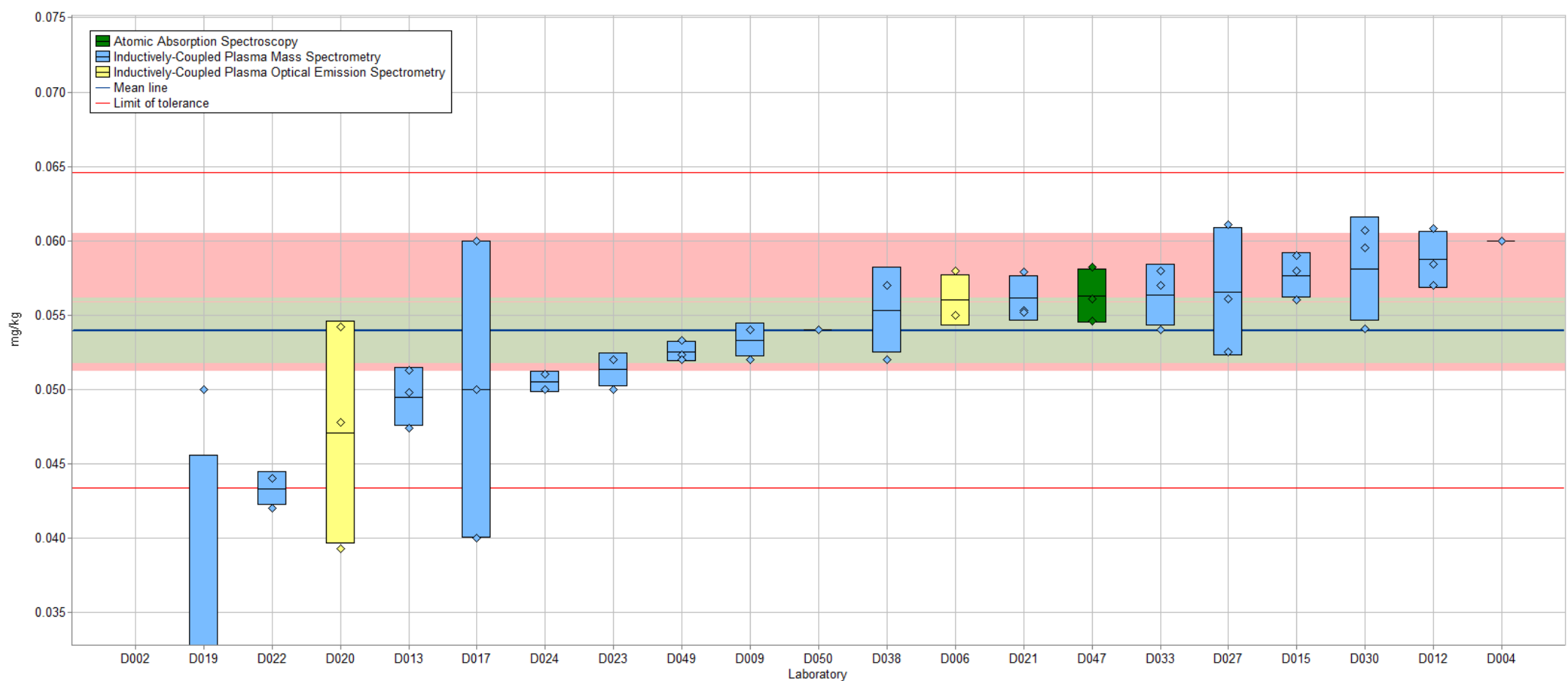

Figure 2-3. Cadmium in SRM 2387 Peanut Butter (data summary view - analytical method). In this view, individual laboratory data are plotted (diamonds) with the individual laboratory standard deviation (rectangle). The color of the data point represents the analytical method employed. The solid blue line represents the consensus mean, and the green shaded region represents the $95 \%$ confidence interval for the consensus mean. The solid red lines represent the consensus range of tolerance, calculated as the values above and below

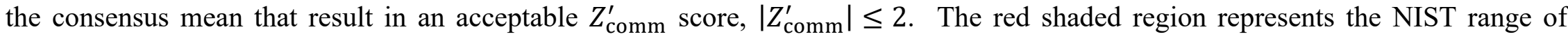
tolerance, which encompasses the target value bounded by twice its uncertainty ( $\left.U_{\mathrm{NIST}}\right)$ and represents the range that results in an acceptable $Z_{\text {NIST }}$ score, $\left|Z_{\text {NIST }}\right| \leq 2$. 


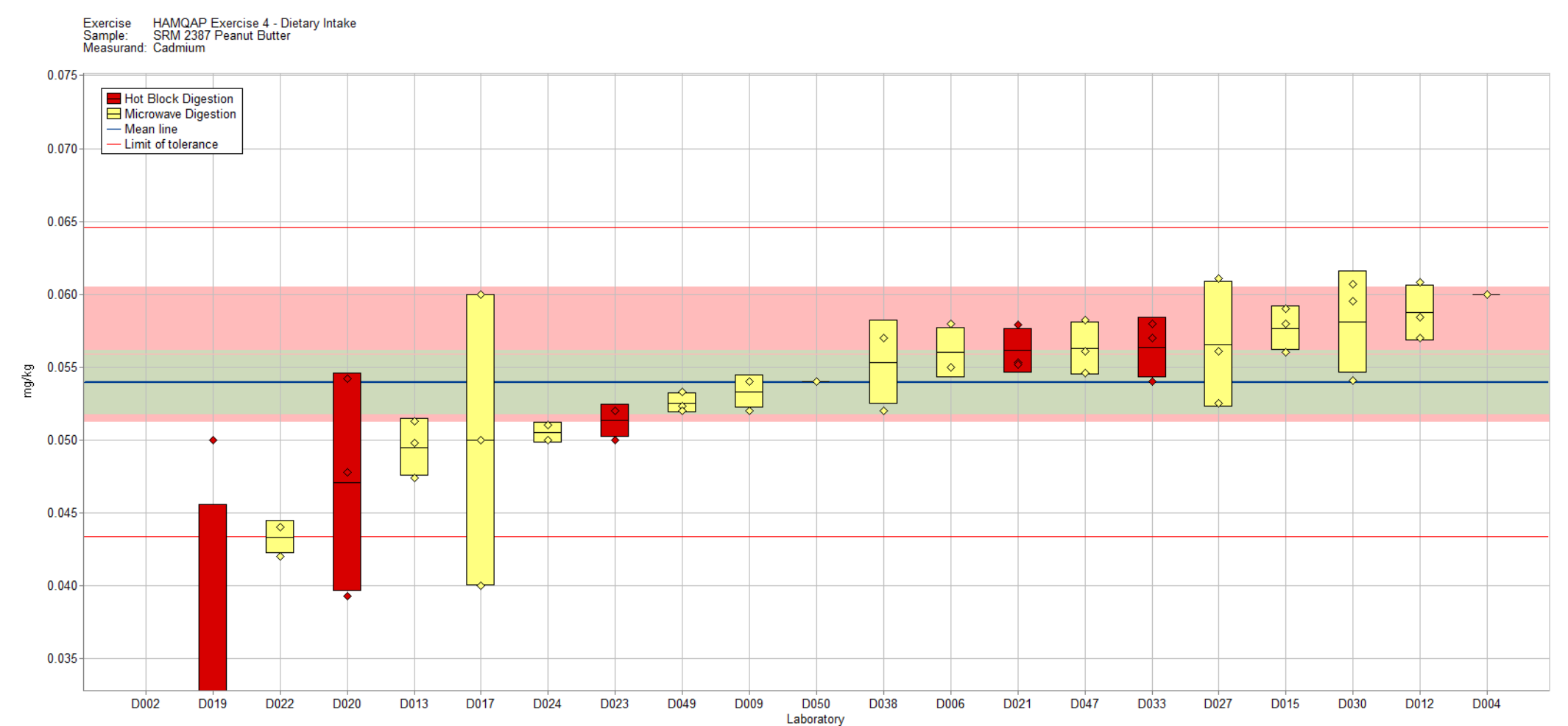

Figure 2-4. Cadmium in SRM 2387 Peanut Butter (data summary view -sample preparation method). In this view, individual laboratory data are plotted (diamonds) with the individual laboratory standard deviation (rectangle). The color of the data point represents the sample preparation method employed. The solid blue line represents the consensus mean, and the green shaded region represents the $95 \%$ confidence interval for the consensus mean. The solid red lines represent the consensus range of tolerance, calculated as the values above and below the consensus mean that result in an acceptable $Z_{\text {comm }}^{\prime}$ score, $\left|Z_{\text {comm }}^{\prime}\right| \leq 2$, with the lower range set at zero. The red shaded region represents the NIST range of tolerance, which encompasses the target value bounded by twice its uncertainty $\left(U_{\text {NIST }}\right)$ and represents the range that results in an acceptable $Z_{\mathrm{NIST}}$ score, $\left|Z_{\mathrm{NIST}}\right| \leq 2$. 


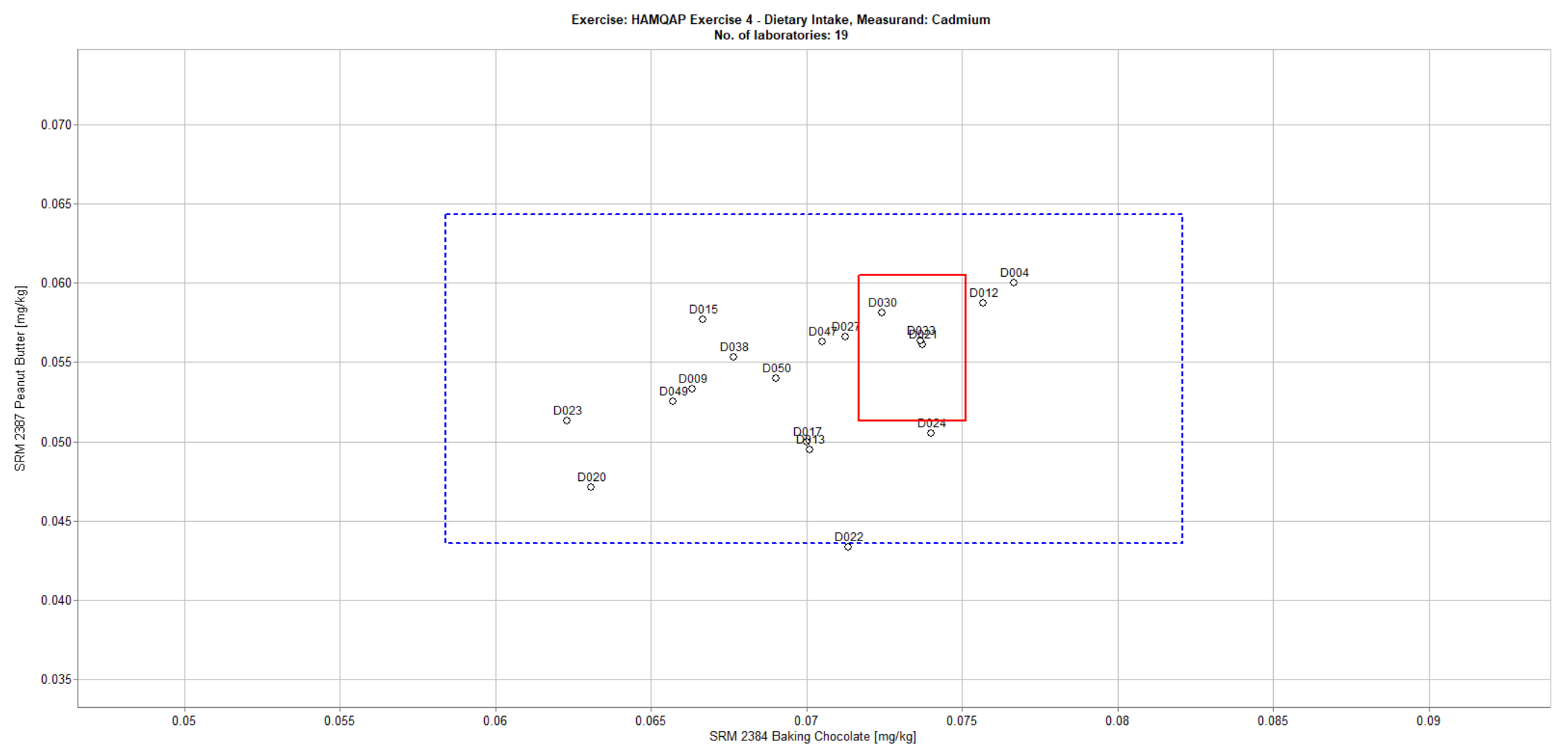

Figure 2-5. Laboratory means for cadmium in SRM 2384 Baking Chocolate and SRM 2387 Peanut Butter (sample/sample comparison view). In this view, the individual laboratory mean for one sample (SRM 2384) is compared to the mean for a second sample (SRM 2387). The solid red box represents the NIST range of tolerance for the two samples, SRM 2384 (x-axis) and SRM 2387 (y-axis), which encompasses the target values bounded by their uncertainties ( $\left.U_{\mathrm{NIST}}\right)$ and represents the range that results in an acceptable $Z_{\mathrm{NIST}}$ score, $\left|Z_{\mathrm{NIST}}\right| \leq 2$. The dotted blue box represents the consensus range of tolerance for SRM 2384 (x-axis) and SRM 2387 (y-axis), calculated as the values above and below the consensus means that result in an acceptable $Z_{\text {comm }}^{\prime}$ score, $\left|Z_{\text {comm }}^{\prime}\right| \leq 2$. 
Table 2-3. Data summary table for lead in baking chocolate and peanut butter. Data points highlighted in red have been flagged as potential outliers (e.g., Grubb and/or Cochran) by the NIST software package.

\begin{tabular}{|c|c|c|c|c|c|c|c|c|c|c|c|}
\hline & & \multicolumn{10}{|c|}{ Lead } \\
\hline & & \multicolumn{5}{|c|}{ SRM 2384 Baking Chocolate (mg/kg) } & \multicolumn{5}{|c|}{ SRM 2387 Peanut Butter (mg/kg) } \\
\hline & Lab & $\mathbf{A}$ & $\mathbf{B}$ & $\mathbf{C}$ & Avg & SD & $\mathbf{A}$ & $\mathbf{B}$ & $\mathbf{C}$ & Avg & SD \\
\hline \multirow{31}{*}{ 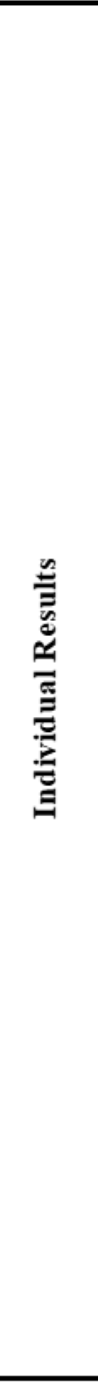 } & Target & & & & 0.0357 & 0.0046 & & & & 0.0023 & 0.0013 \\
\hline & D001 & & & & & & & & & & \\
\hline & D002 & $<0.010$ & $<0.010$ & $<0.010$ & & & $<0.010$ & $<0.010$ & $<0.010$ & & \\
\hline & D004 & 0.05 & 0.04 & 0.04 & 0.0433 & 0.0058 & 0.01 & 0.01 & 0.01 & 0.0100 & 0.0000 \\
\hline & D005 & & & & & & & & & & \\
\hline & D006 & & & & & & & & & & \\
\hline & D007 & & & & & & & & & & \\
\hline & D009 & 0.032 & 0.037 & 0.035 & 0.0347 & 0.0025 & 0.003 & 0.004 & 0.003 & 0.0033 & 0.0006 \\
\hline & D010 & & & & & & & & & & \\
\hline & D012 & 0.0337 & 0.0327 & 0.0343 & 0.0336 & 0.0008 & 0.0048 & 0.0012 & 0.0007 & 0.0022 & 0.0022 \\
\hline & D013 & 0.0351 & 0.0361 & 0.0387 & 0.0366 & 0.0019 & $<0.003$ & $<0.003$ & $<0.003$ & & \\
\hline & D015 & 0.038 & 0.038 & 0.036 & 0.0373 & 0.0012 & 0.0083 & 0.0086 & 0.0053 & 0.0074 & 0.0018 \\
\hline & D016 & 0.02772 & 0.02664 & 0.02718 & 0.0272 & 0.0005 & & & & & \\
\hline & D017 & 0.04 & 0.04 & 0.04 & 0.0400 & 0.0000 & $<0.040$ & $<0.040$ & $<0.040$ & & \\
\hline & D019 & & 0.02 & 0.03 & 0.0250 & 0.0071 & 0.0005 & & & 0.0005 & \\
\hline & D020 & 0.0378 & 0.0294 & 0.0404 & 0.0359 & 0.0058 & 0.004 & 0.0028 & 0.0042 & 0.0037 & 0.0008 \\
\hline & D021 & 0.0534 & 0.0391 & 0.0331 & 0.0419 & 0.0104 & $<0.023$ & $<0.023$ & $<0.023$ & & \\
\hline & D022 & 0.03 & 0.029 & 0.029 & 0.0293 & 0.0006 & 0.003 & 0.004 & 0.003 & 0.0033 & 0.0006 \\
\hline & D023 & 0.0267 & 0.0269 & 0.0297 & 0.0278 & 0.0017 & 0.006 & 0.01 & 0.004 & 0.0067 & 0.0031 \\
\hline & D024 & 0.12 & 0.121 & & 0.1205 & 0.0007 & 0.005 & 0.005 & & 0.0050 & 0.0000 \\
\hline & D027 & 0.0332 & 0.033 & 0.0313 & 0.0325 & 0.0010 & 0.0021 & 0.0028 & 0.018 & 0.0076 & 0.0090 \\
\hline & D030 & 0.0331 & 0.0359 & 0.0397 & 0.0362 & 0.0033 & 0.0021 & 0.0017 & 0.0015 & 0.0018 & 0.0003 \\
\hline & D033 & 0.03 & $<0.030$ & $<0.030$ & 0.0300 & & $<0.030$ & $<0.030$ & $<0.030$ & & \\
\hline & D034 & & & & & & & & & & \\
\hline & D036 & $<0.050$ & $<0.050$ & $<0.050$ & & & $<0.050$ & $<0.050$ & $<0.050$ & & \\
\hline & D041 & & & & & & & & & & \\
\hline & D045 & & & & & & & & & & \\
\hline & D046 & & & & & & & & & & \\
\hline & D047 & 0.0409 & 0.0454 & 0.0371 & 0.0411 & 0.0042 & & & & & \\
\hline & D049 & 0.0316 & 0.0302 & 0.0278 & 0.0299 & 0.0019 & $<0.005$ & $<0.005$ & $<0.005$ & & \\
\hline & D050 & 0.035 & 0.033 & 0.04 & 0.0360 & 0.0036 & 0.002 & 0.002 & 0.002 & 0.0020 & 0.0000 \\
\hline 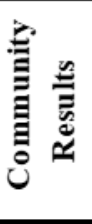 & & $\begin{array}{l}\text { Consensu } \\
\text { Consensu } \\
\text { Maximum } \\
\text { Minimum } \\
\mathrm{N} \\
\end{array}$ & $\begin{array}{l}\text { is Mean } \\
\text { ss Standarc }\end{array}$ & Deviation & $\begin{array}{c}0.0344 \\
0.0014 \\
0.1205 \\
0.0250 \\
18 \\
\end{array}$ & & $\begin{array}{l}\text { Consensu } \\
\text { Consensu } \\
\text { Maximum } \\
\text { Minimum } \\
\mathrm{N} \\
\end{array}$ & $\begin{array}{l}\text { s Mean } \\
\text { s Standard }\end{array}$ & Deviation & $\begin{array}{c}0.0043 \\
0.0008 \\
0.0100 \\
0.0005 \\
11 \\
\end{array}$ & \\
\hline
\end{tabular}




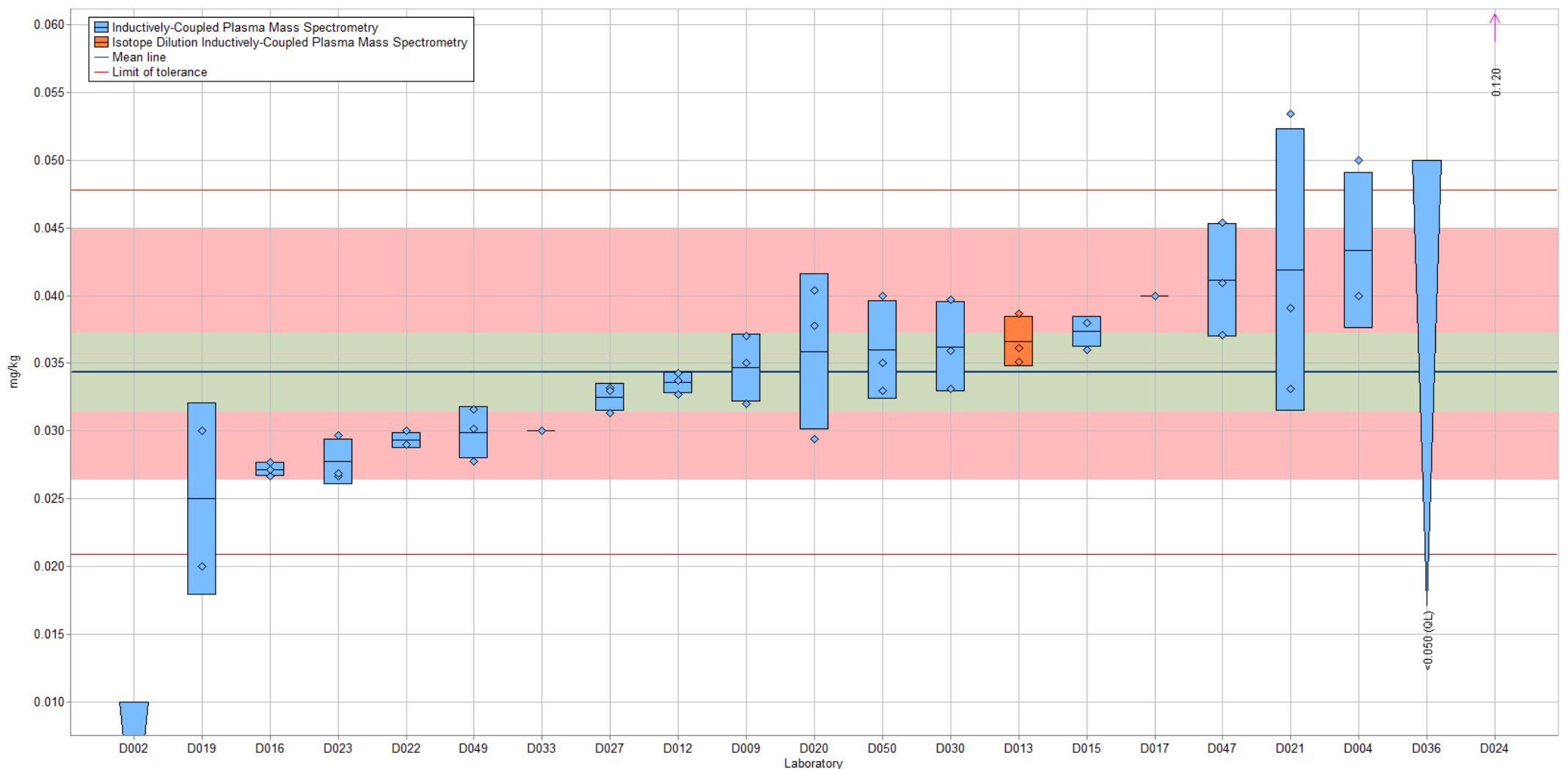

Figure 2-6. Lead in SRM 2384 Baking Chocolate (data summary view - analytical method). In this view, individual laboratory data are plotted (diamonds) with the individual laboratory standard deviation (rectangle). The color of the data point represents the analytical method employed. The solid blue line represents the consensus mean, and the green shaded region represents the $95 \%$ confidence interval for the consensus mean. The solid red lines represent the consensus range of tolerance, calculated as the values above and below the consensus mean that result in an acceptable $Z_{\text {comm }}^{\prime}$ score, $\left|Z_{\text {comm }}^{\prime}\right| \leq 2$. The red shaded region represents the NIST range of tolerance, which encompasses the target value bounded by twice its uncertainty ( $\left.U_{\text {NIST }}\right)$ and represents the range that results in an acceptable $Z_{\text {NIST }}$ score, $\left|Z_{\text {NIST }}\right| \leq 2$. 


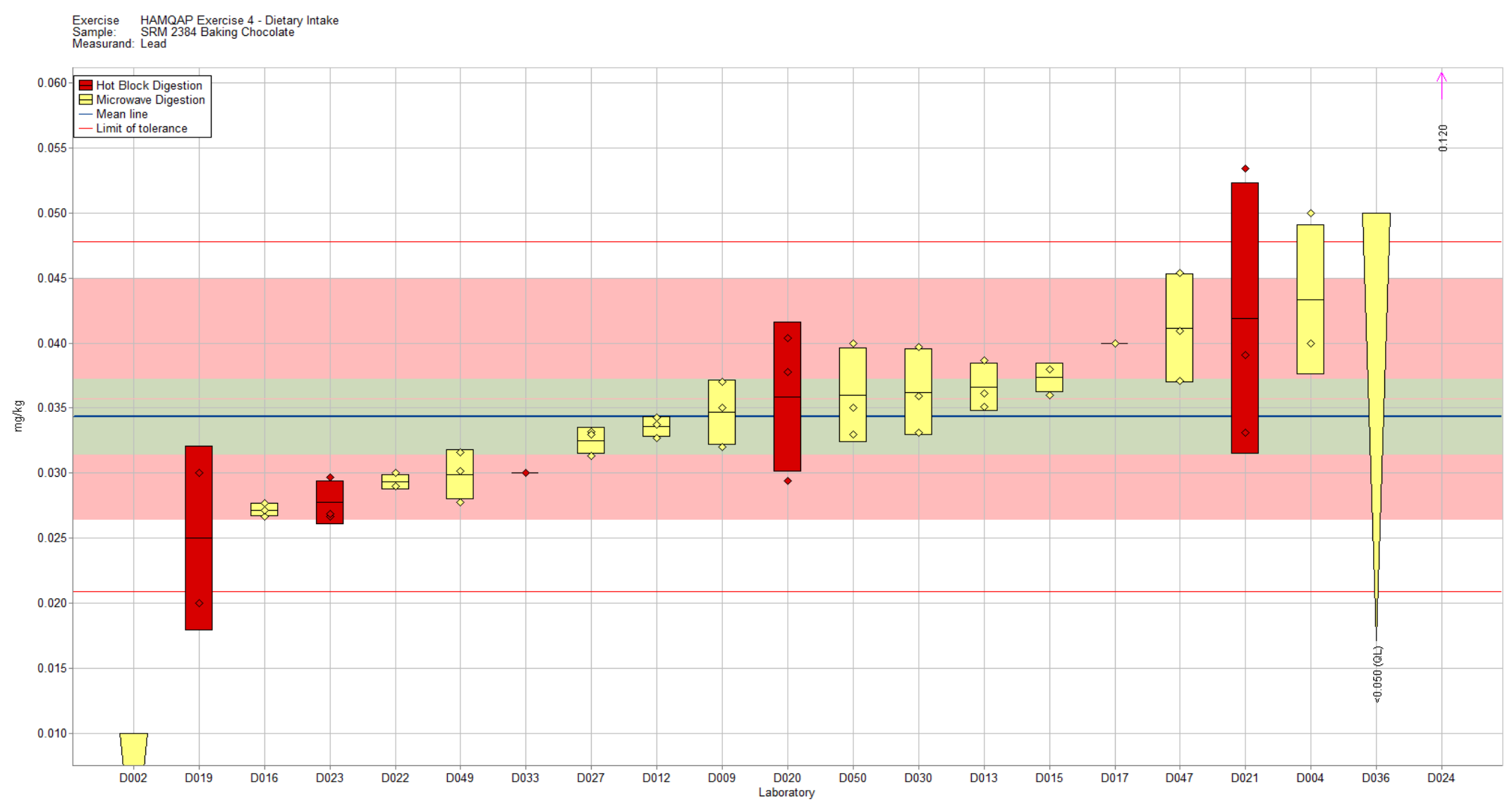

Figure 2-7. Lead in SRM 2384 Baking Chocolate (data summary view - sample preparation method). In this view, individual laboratory data are plotted (diamonds) with the individual laboratory standard deviation (rectangle). The color of the data point represents the sample preparation method employed. The solid blue line represents the consensus mean, and the green shaded region represents the $95 \%$ confidence interval for the consensus mean. The solid red lines represent the consensus range of tolerance, calculated as the values above and below the consensus mean that result in an acceptable $Z_{\text {comm }}^{\prime}$ score, $\left|Z_{\text {comm }}^{\prime}\right| \leq 2$. The red shaded region represents the NIST range of tolerance, which encompasses the target value bounded by twice its uncertainty $\left(U_{\text {NIST }}\right)$ and represents the range that results in an acceptable $Z_{\text {NIST }}$ score, $\left|Z_{\text {NIST }}\right| \leq 2$. 


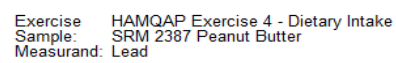

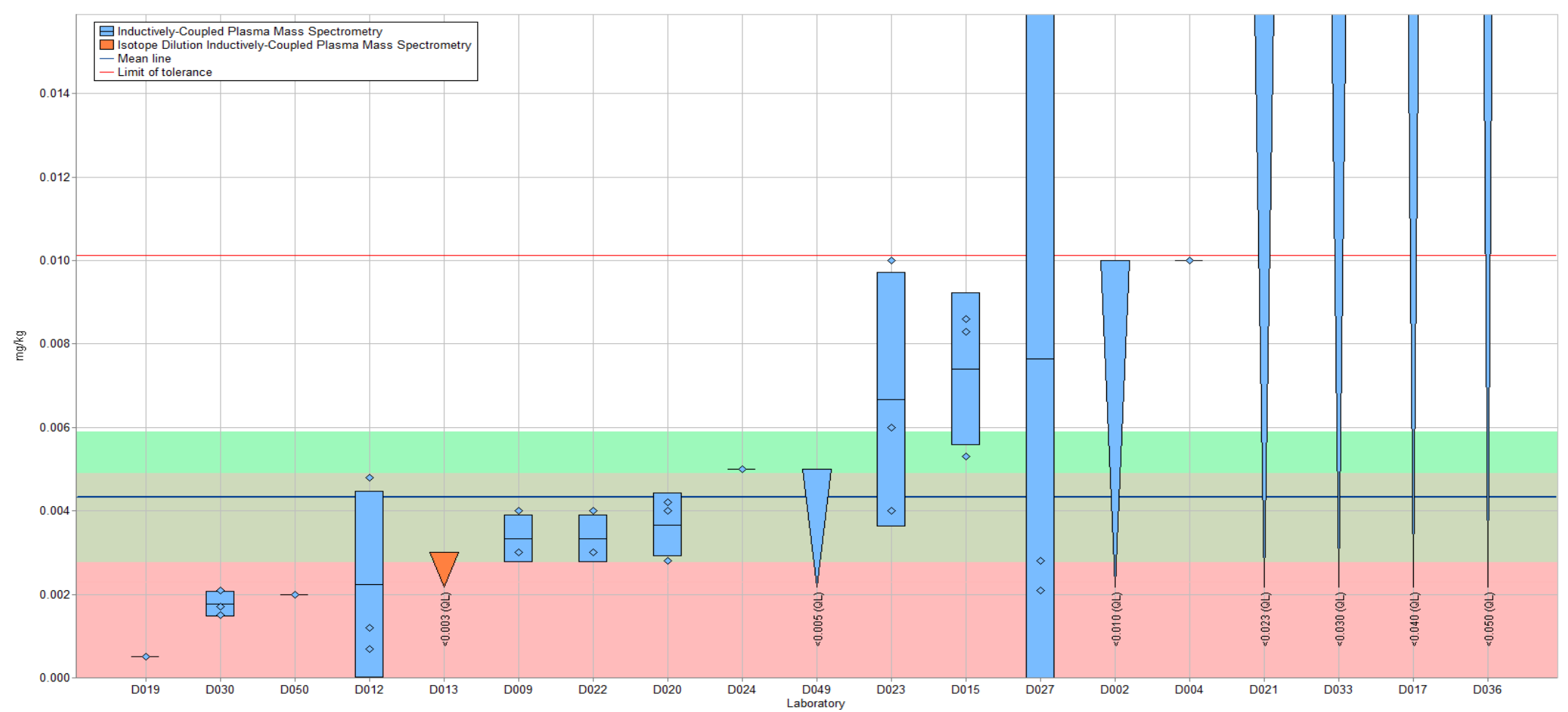

Figure 2-8. Lead in SRM 2387 Peanut Butter (data summary view - analytical method). In this view, individual laboratory data are plotted (diamonds) with the individual laboratory standard deviation (rectangle). The color of the data point represents the analytical method employed. The solid blue line represents the consensus mean, and the green shaded region represents the $95 \%$ confidence interval for the consensus mean. The solid red line represents the upper consensus range of tolerance, calculated as the values above the consensus mean that result in an acceptable $Z_{\text {comm }}^{\prime}$ score, $\left|Z_{\text {comm }}^{\prime}\right| \leq 2$, with the lower limit set at zero. The red shaded region represents the NIST range of tolerance, which encompasses the target value bounded by twice its uncertainty ( $\left.U_{\text {NIST }}\right)$ and represents the range that results in an acceptable $Z_{\mathrm{NIST}}$ score, $\left|Z_{\mathrm{NIST}}\right| \leq 2$. 
Exercise
SAMMAPQPe: Exercise 4 - Dietary Intake
Measurand: Lead 2387 Peanut Butter

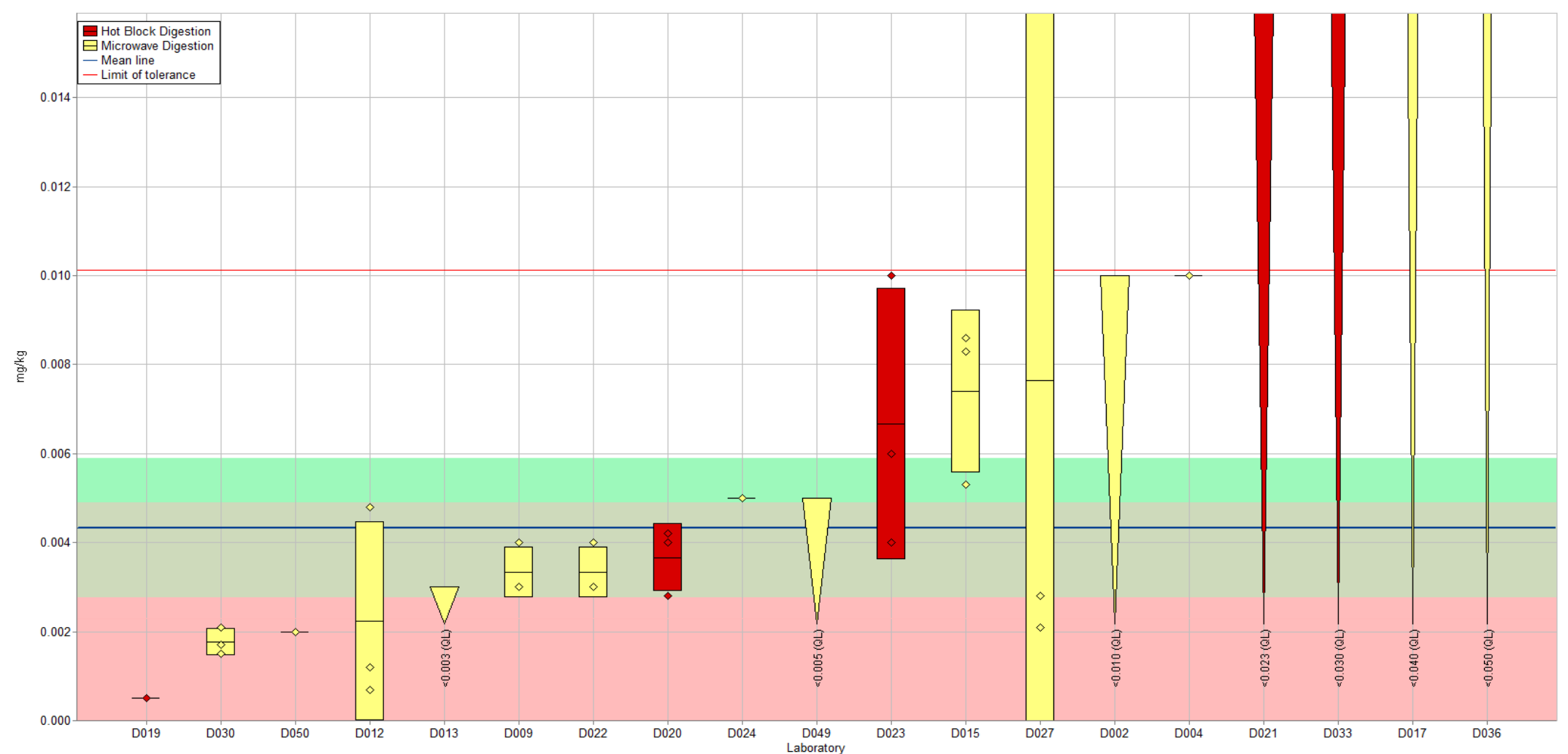

Figure 2-9. Lead in SRM 2387 Peanut Butter (data summary view - sample preparation method). In this view, individual laboratory data are plotted (diamonds) with the individual laboratory standard deviation (rectangle). The color of the data point represents the sample preparation method employed. The solid blue line represents the consensus mean, and the green shaded region represents the $95 \%$ confidence interval for the consensus mean. The solid red line represents the upper consensus range of tolerance, calculated as the values above the consensus mean that result in an acceptable $Z_{\text {comm }}^{\prime}$ score, $\left|Z_{\text {comm }}^{\prime}\right| \leq 2$, with the lower limit set at zero. The red shaded region represents the NIST range of tolerance, which encompasses the target value bounded by twice its uncertainty ( $\left.U_{\mathrm{NIST}}\right)$ and represents the range that results in an acceptable $Z_{\text {NIST }}$ score, $\left|Z_{\text {NIST }}\right| \leq 2$. 


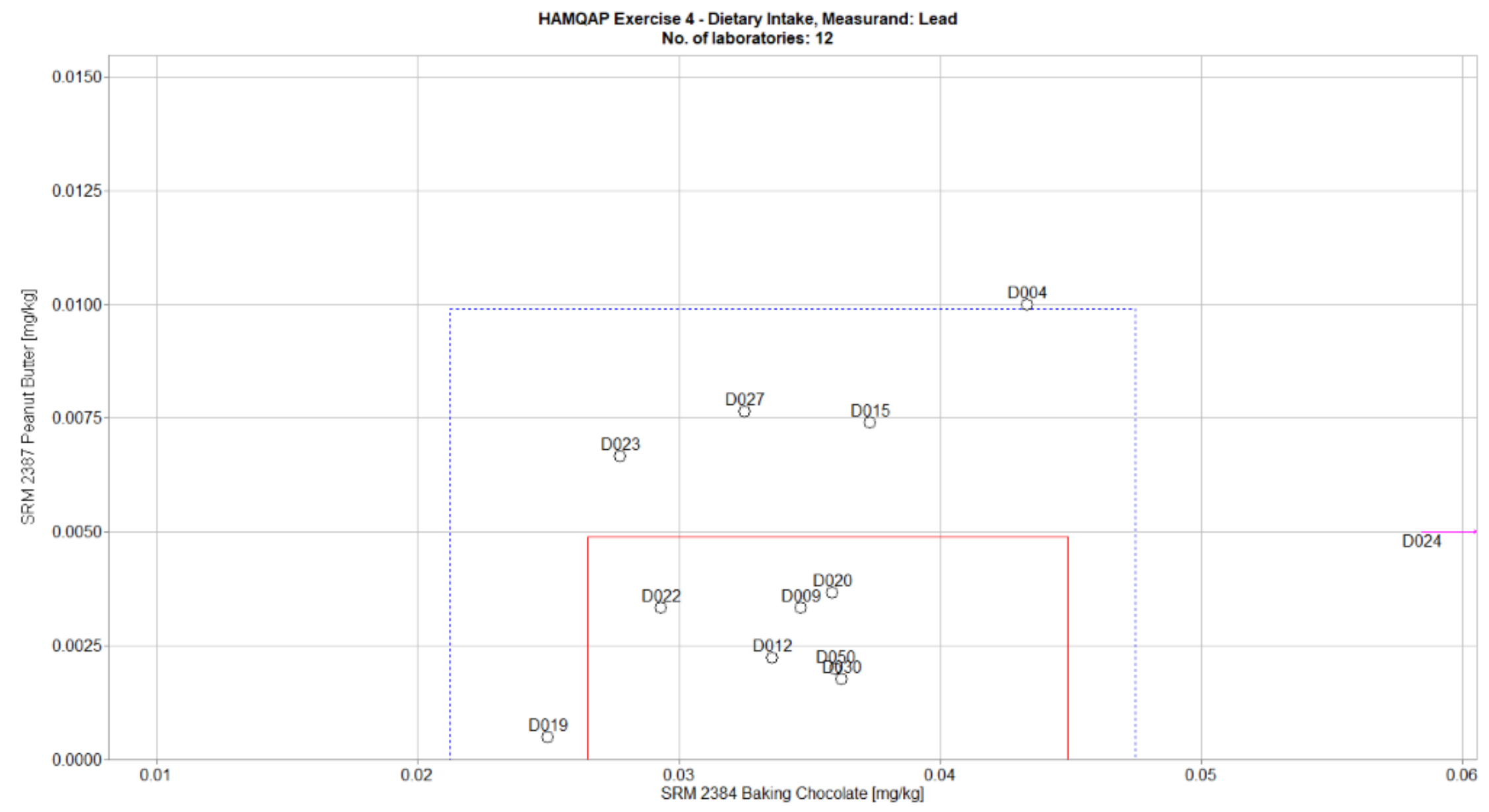

Figure 2-10. Laboratory means for lead in SRM 2384 Baking Chocolate and SRM 2387 Peanut Butter (sample/sample comparison view). In this view, the individual laboratory mean for one sample (SRM 2384) is compared to the mean for a second sample (SRM 2387). The solid red box represents the NIST range of tolerance for the two samples, SRM 2384 (x-axis) and SRM 2387 (y-axis), which encompasses the target values bounded by their uncertainties ( $\left.U_{\mathrm{NIST}}\right)$ and represents the range that results in an acceptable $Z_{\mathrm{NIST}}$ score, $\left|Z_{\mathrm{NIST}}\right| \leq 2$. The dotted blue box represents the consensus range of tolerance for SRM 2384 (x-axis) and SRM 2387 (y-axis), calculated as the values above and below the consensus means that result in an acceptable $Z_{\text {comm }}^{\prime}$ score, $\left|Z_{\text {comm }}^{\prime}\right| \leq 2$. 


\section{SECTION 3: WATER-SOLUBLE VITAMINS (Vitamin $B_{12}$ )}

\section{$\underline{\text { Study Overview }}$}

In this study, participants were provided with samples of SRM 1869 Infant/Adult Nutritional Formula II and multivitamin tablets for dietary intake. Participants were asked to use in-house analytical methods to determine the mass fraction $(\mathrm{mg} / \mathrm{kg})$ of vitamin $\mathrm{B}_{12}$ in each matrix. Vitamin $\mathrm{B}_{12}$ is necessary for the health of human nerve and blood cells, is involved in DNA synthesis, and prevents megaloblastic anemia. ${ }^{9}$ Human intake of vitamin $\mathrm{B}_{12}$ is primarily from consumption of animal-sourced foods (fish, meat, poultry, eggs, milk), fortified foods (breakfast cereals, nutritional yeasts), or from supplementation (most multivitamins contain vitamin $\mathrm{B}_{12}$ ). Accurate understanding vitamin $\mathrm{B}_{12}$ intake through measurement in supplements and fortified foods, as well as the comparability of various approaches to estimating vitamin $\mathrm{B}_{12}$ health status, can inform future decisions about recommended dietary intakes.

\section{Dietary Intake Sample Information}

Infant Formula. Participants were provided with three packets, each containing approximately $10 \mathrm{~g}$ of powdered material. Participants were asked to store the material at $-20{ }^{\circ} \mathrm{C}$ in the original unopened packet and to prepare one sample and report one value from each packet provided. Before use, participants were instructed to thoroughly mix the contents of the packet prior to removal of a test portion for analysis, and to use a sample size of at least $1 \mathrm{~g}$. The approximate analyte levels were not reported to participants prior to the study. A reference value for vitamin $\mathrm{B}_{12}$ in SRM 1869 was assigned using results from collaborating laboratories and the manufacturer of the material. The reference value and uncertainty for vitamin B12 in SRM 1869 are provided in the table below on an as-received basis.

\section{Analyte $\quad$ Reference Mass Fraction in SRM $1869(\mathrm{mg} / \mathrm{kg})$ \\ Vitamin $\mathrm{B}_{12} \quad 0.0435 \pm 0.0065$}

Multivitamin. Participants were provided with three bottles, each containing 30 multivitamin tablets. Participants were asked to store the material at controlled room temperature, $20{ }^{\circ} \mathrm{C}$ to $25^{\circ} \mathrm{C}$, in the original unopened bottles and to prepare one sample and report one value from each bottle provided. Before use, participants were instructed to grind all 30 tablets and mix the resulting powder thoroughly prior to removal of a test portion for analysis, and to use a sample size of at least $0.3 \mathrm{~g}$. Approximate analyte levels were not reported to participants prior to the study. The NIST-determined value for vitamin $\mathrm{B}_{12}$ in the multivitamin sample was assigned using results from the manufacturer of the material. The NIST-determined value and uncertainty for vitamin $\mathrm{B}_{12}$ are provided in the table below on an as-received basis.

$\frac{\text { Analyte }}{\text { Vitamin } \mathrm{B}_{12}} \quad \frac{\text { NIST-Determined Mass Fraction in Multivitamin }(\mathrm{mg} / \mathrm{kg})}{5.78 \pm 0.22}$

\footnotetext{
${ }^{9}$ Vitamin $B_{12}$ Fact Sheet for Health Professionals. National Institutes of Health Office of Dietary Supplements. https://ods.od.nih.gov/factsheets/VitaminB12-healthprofessional/ (accessed February 2020).
} 
Dietary Intake Study Results

- Twenty-five laboratories enrolled in this exercise and received samples to measure vitamin $\mathrm{B}_{12}$.

- Eight laboratories reported results for vitamin $\mathrm{B}_{12}$ in the infant formula (32\% participation), and 15 laboratories reported results for vitamin $\mathrm{B}_{12}$ in the multivitamin (60\% participation).

- For both infant formula and the multivitamin, the consensus mean for vitamin $\mathrm{B}_{12}$ was within the target range. The between-laboratory variability was good for both materials, with $15 \%$ RSD for the infant formula and $8 \%$ RSD for the multivitamin (Table 3-2, Figure 3-1 and Figure 3-2, respectively).

- Most laboratories reported using LC-absorbance or LC-MS methods for determination of vitamin $\mathrm{B}_{12}$ (see table below).

\begin{tabular}{|c|c|c|}
\hline \multirow{3}{*}{$\frac{\text { Analytical Method }}{\underline{\text { Reported }}}$} & \multicolumn{2}{|c|}{ Number of Laboratories Reporting Use of Metho } \\
\hline & & \\
\hline & $\underline{\text { SRM } 1869}$ & $\underline{\text { Multivitamin }}$ \\
\hline LC-Absorbance & $4(50 \%)$ & $11(73 \%)$ \\
\hline LC-MS & $3(38 \%)$ & $3(20 \%)$ \\
\hline Microbiological Assay & $1(12 \%)$ & $1(7 \%)$ \\
\hline
\end{tabular}

- Most laboratories reported using solvent extraction or dilution in the preparation of samples for determination of vitamin $\mathrm{B}_{12}$ (see table below).

\author{
Sample Preparation \\ Method Reported \\ Solvent Extraction
}

Dilution

Solid Phase Extraction

None/Other
Number of Laboratories Reporting Use of Method (Percent of Results Reported Using Method)

\begin{tabular}{cc} 
SRM 1869 & Multivitamin \\
\hline $3(38 \%)$ & $8(53 \%)$ \\
$2(25 \%)$ & $3(20 \%)$ \\
$1(12 \%)$ & $2(13 \%)$ \\
$2(25 \%)$ & $2(13 \%)$
\end{tabular}

Dietary Intake Technical Recommendations

The following recommendations are based on results obtained from the participants in this study.

- As shown in Figure 3-1, half of the laboratories reported values for vitamin $\mathrm{B}_{12}$ that were within the NIST range of tolerance for SRM 1869 Infant/Adult Nutritional Formula II. Figure 3-2 shows fewer laboratories (20\%) overlap the NIST range of tolerance for the multivitamin; however, 7 of the 15 reported values for the multivitamin were within the $95 \%$ confidence interval for the consensus mean.

- Figure 3-3 shows that for the four laboratories that returned results for both samples, all values for the multivitamin samples were below the NIST target value. All laboratories used different sample preparation approaches and used the three different analytical methods, indicating a challenge with the material itself.

- Prior to extraction, laboratories may not have properly ground and homogenized the tablets, resulting in lower than expected results for vitamin $\mathrm{B}_{12}$. 
- The multivitamin material may be challenging for laboratories to prepare and accurately analyze. Methods should be evaluated using control materials (CRMs, RMs, etc.) before analyzing unknown materials to ensure acceptable performance.

- Three laboratories reported values in the infant formula that were significantly outside the acceptable range of twice the upper limit of tolerance. Two of these laboratories used different detection techniques (LC-absorbance and LC-MS) which suggests the discrepancy in the reported values is more likely a result of the extraction procedure and not the detection technique. Additional information is needed to make specific recommendations, including an understanding of the extraction procedure and calibration approach, but these laboratories should review their methods carefully for potential biases.

- Vitamin B12 may decompose in light, and therefore samples and standards should be prepared in a room with amber or attenuated lighting.

- The calculations and reporting units must be verified prior to submission. For example, three laboratories reported results that are multiple orders of magnitude higher than the target value, which indicates results reported in the wrong units or a dilution factor may have been forgotten during the calculation of the final results.

- The use of appropriate calibration materials and quality assurance samples to establish that a method is in control and being performed correctly may reduce the likelihood of outlying data. Quality assurance samples can be commercially available reference materials (CRMs, SRMs, or RMs) or materials prepared in-house. 
Table 3-1. Individualized data summary table (NIST) for vitamin $\mathrm{B}_{12}$ in infant formula and multivitamin.

\section{National Institute of Standards \& Technology}

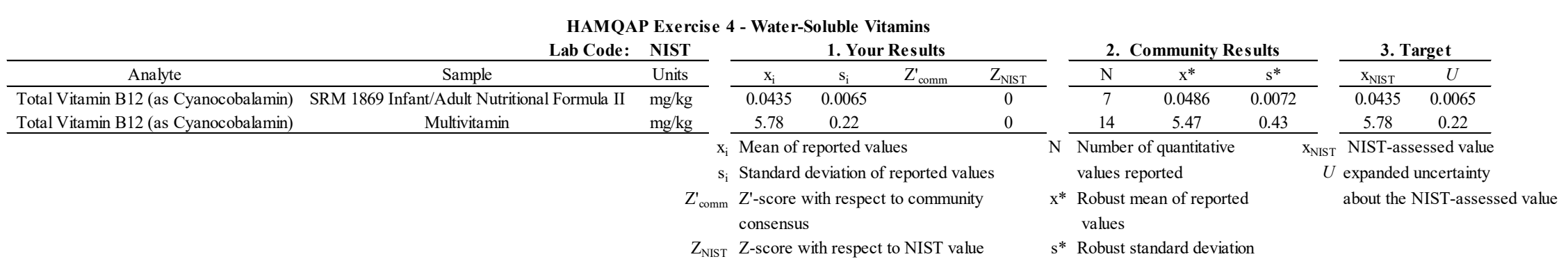


Table 3-2. Data summary table for vitamin $B_{12}$ in infant formula and multivitamin. Data points highlighted in red have been flagged as potential outliers (e.g., Grubb and/or Cochran) by the NIST software package.

\begin{tabular}{|c|c|c|c|c|c|c|c|c|c|c|c|}
\hline & & \multicolumn{10}{|c|}{ Total Vitamin $\mathbf{B}_{12}$ (as Cyanocobalamin) } \\
\hline & & \multicolumn{5}{|c|}{$\begin{array}{l}\text { SRM } 1869 \text { Infant/Adult Nutritional Formula II } \\
(\text { milk/whey/soy-based })(\mathrm{mg} / \mathrm{kg})\end{array}$} & \multicolumn{5}{|c|}{ Multivitamin (mg/kg) } \\
\hline & Lab & $\mathbf{A}$ & $\mathbf{B}$ & $\mathbf{C}$ & Avg & SD & $\mathbf{A}$ & $\mathbf{B}$ & $\mathbf{C}$ & Avg & SD \\
\hline \multirow{26}{*}{ 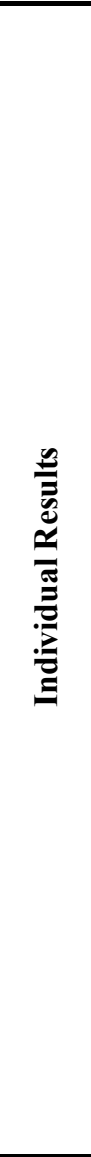 } & Target & & & & 0.0435 & 0.0065 & & & & 5.78 & 0.22 \\
\hline & D001 & & & & & & 5.51 & 5.22 & 6.5 & 5.74 & 0.67 \\
\hline & D004 & 23.43 & 21.32 & 23.49 & 22.7467 & 1.2359 & 2318 & 2348 & 2332 & 2333 & 15 \\
\hline & D005 & & & & & & & & & & \\
\hline & D006 & & & & & & & & & & \\
\hline & D007 & & & & & & & & & & \\
\hline & D009 & & & & & & 6.75 & 6.17 & 5.99 & 6.30 & 0.40 \\
\hline & D010 & & & & & & 7.16 & 9.4 & 8.45 & 8.34 & 1.12 \\
\hline & D011 & & & & & & & & & & \\
\hline & D013 & & & & & & & & & & \\
\hline & D014 & 7.4 & 11 & 10.7 & 9.7000 & 1.9975 & 8.8 & 8.02 & 7.48 & 8.10 & 0.66 \\
\hline & D017 & & & & & & & & & & \\
\hline & D018 & & & & & & & & & & \\
\hline & D019 & & & & & & 4.6332 & 5.197 & 4.952 & 4.93 & 0.28 \\
\hline & D021 & 0.0515 & 0.0531 & 0.0573 & 0.0540 & 0.0030 & 4.22 & 4.5 & 4.82 & 4.51 & 0.30 \\
\hline & D023 & $<0.500$ & $<0.500$ & $<0.500$ & & & $<0.500$ & $<0.500$ & $<0.500$ & & \\
\hline & D024 & 0.948 & 0.996 & 1.05 & 0.9980 & 0.0510 & 7.09 & 7.2 & 6.64 & 6.98 & 0.30 \\
\hline & D026 & 0.0491 & 0.0492 & 0.0485 & 0.0489 & 0.0004 & 3.75 & 4.64 & 4.81 & 4.40 & 0.57 \\
\hline & D031 & & & & & & 4.37 & 4.17 & 4.57 & 4.37 & 0.20 \\
\hline & D034 & & & & & & & & & & \\
\hline & D035 & & & & & & & & & & \\
\hline & D036 & 0.05 & 0.04 & 0.04 & 0.0433 & 0.0058 & 4.36 & 4.44 & 4.39 & 4.40 & 0.04 \\
\hline & D046 & & & & & & & & & & \\
\hline & D048 & & & & & & 4.645 & 4.441 & 4.421 & 4.50 & 0.12 \\
\hline & D049 & 0.0481 & 0.0479 & 0.049 & 0.0483 & 0.0006 & 3.88 & 3.67 & 3.6 & 3.72 & 0.15 \\
\hline & D050 & & & & & & 5.34 & 5.34 & 5.53 & 5.40 & 0.11 \\
\hline \multirow{5}{*}{ 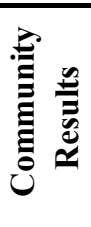 } & & \multicolumn{3}{|c|}{ Consensus Mean } & \multicolumn{2}{|l|}{0.0486} & \multicolumn{3}{|c|}{ Consensus Mean } & 5.47 & \\
\hline & & \multicolumn{3}{|c|}{ Consensus Standard Deviation } & \multicolumn{2}{|l|}{0.0072} & \multicolumn{4}{|c|}{ Consensus Standard Deviation $\quad 0.43$} & \\
\hline & & & & \multicolumn{2}{|l|}{22.7467} & \multicolumn{3}{|c|}{ Maximum } & 2333 & \\
\hline & & \multicolumn{2}{|l|}{ Minimum } & & \multicolumn{2}{|l|}{0.0433} & \multicolumn{2}{|c|}{ Minimum } & & \multirow{2}{*}{$\begin{array}{c}3.72 \\
14\end{array}$} & \\
\hline & & \multicolumn{2}{|l|}{$\mathrm{N}$} & & \multicolumn{2}{|l|}{7} & \multicolumn{2}{|l|}{$\mathrm{N}$} & & & \\
\hline
\end{tabular}


Exercise HAMOAP Exercise 4- Dietary Intake

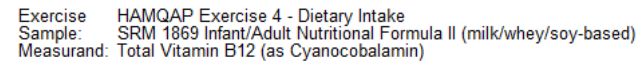

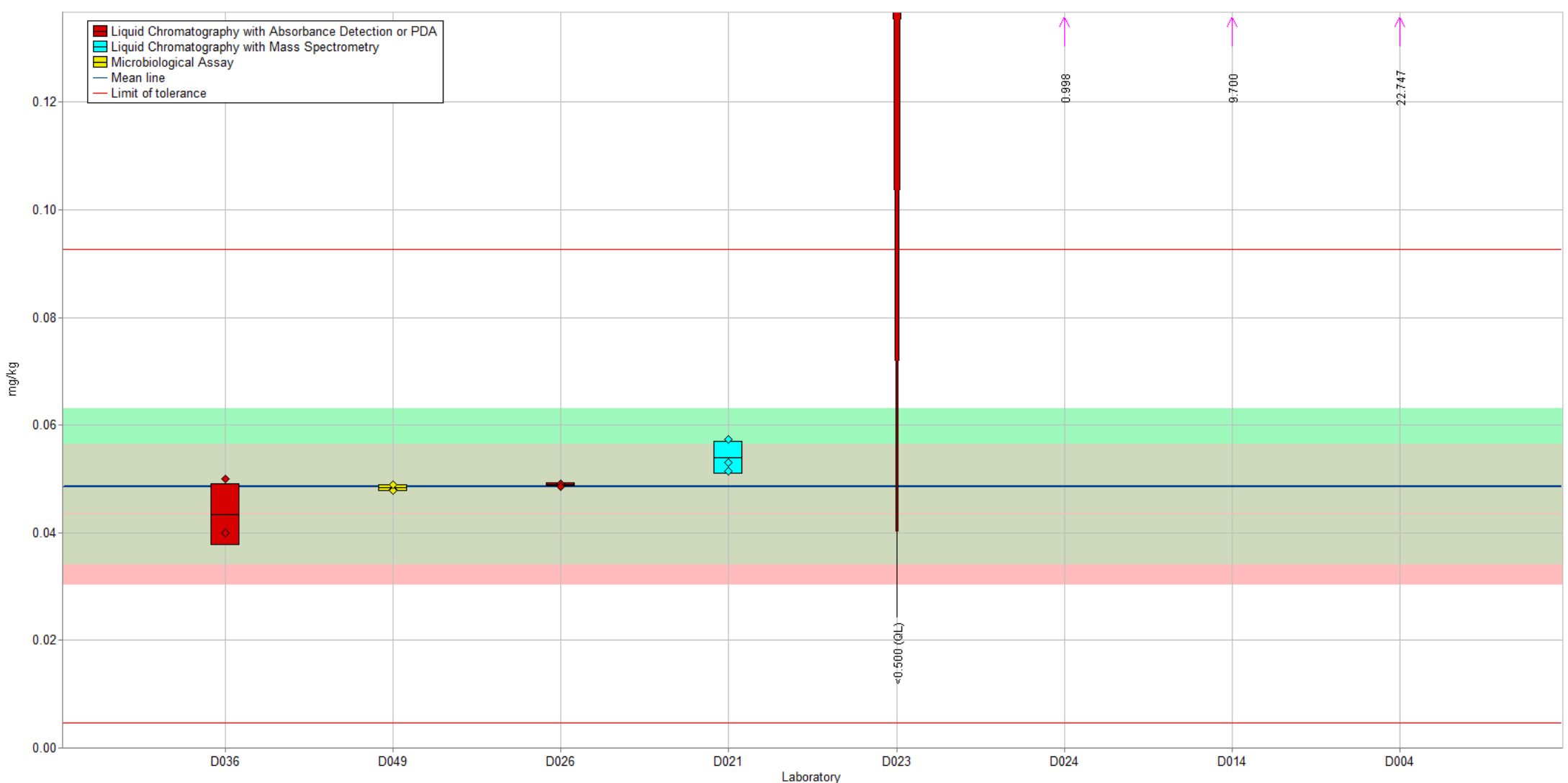

Figure 3-1. Vitamin $B_{12}$ in SRM 1869 Infant/Adult Nutritional Formula II (data summary view - analytical method). In this view, individual laboratory data are plotted (diamonds) with the individual laboratory standard deviation (rectangle). The color of the data point represents the analytical method employed. The solid blue line represents the consensus mean, and the green shaded region represents the $95 \%$ confidence interval for the consensus mean. The solid red lines represent the consensus range of tolerance, calculated as the values above and below the consensus mean that result in an acceptable $Z_{\text {comm }}^{\prime}$ score, $\left|Z_{\text {comm }}^{\prime}\right| \leq 2$. The red shaded region represents the NIST range of tolerance, which encompasses the target value bounded by twice its uncertainty $\left(U_{\text {NIST }}\right)$ and represents the range that results in an acceptable $Z_{\text {NIST }}$ score, $\left|Z_{\text {NIST }}\right| \leq 2$. 
Exercise HAMQAP Exercise 4 - Dietary Intake

Sample: Multiviamin

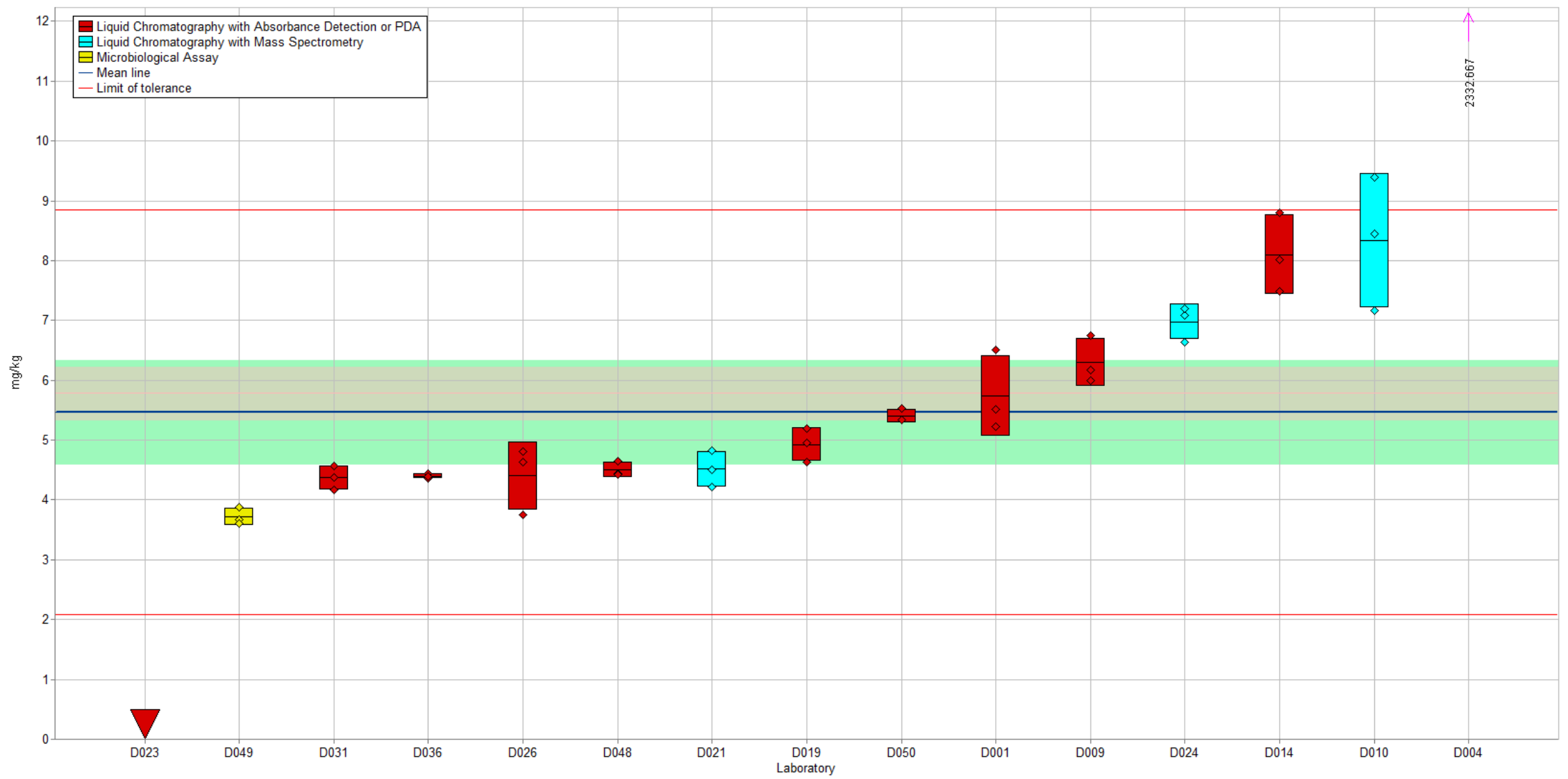

Figure 3-2. Vitamin $B_{12}$ in Multivitamin (data summary view - analytical method). In this view, individual laboratory data are plotted (diamonds) with the individual laboratory standard deviation (rectangle). The color of the data point represents the analytical method employed. The solid blue line represents the consensus mean, and the green shaded region represents the $95 \%$ confidence interval for the consensus mean. The solid red lines represent the consensus range of tolerance, calculated as the values above and below the consensus mean that result in an acceptable $Z_{\text {comm }}^{\prime}$ score, $\left|Z_{\text {comm }}^{\prime}\right| \leq 2$. The red shaded region represents the NIST range of tolerance, which encompasses the target value bounded by twice its uncertainty ( $\left.U_{\text {NIST }}\right)$ and represents the range that results in an acceptable $Z_{\text {NIST }}$ score, $\left|Z_{\text {NIST }}\right| \leq 2$. 


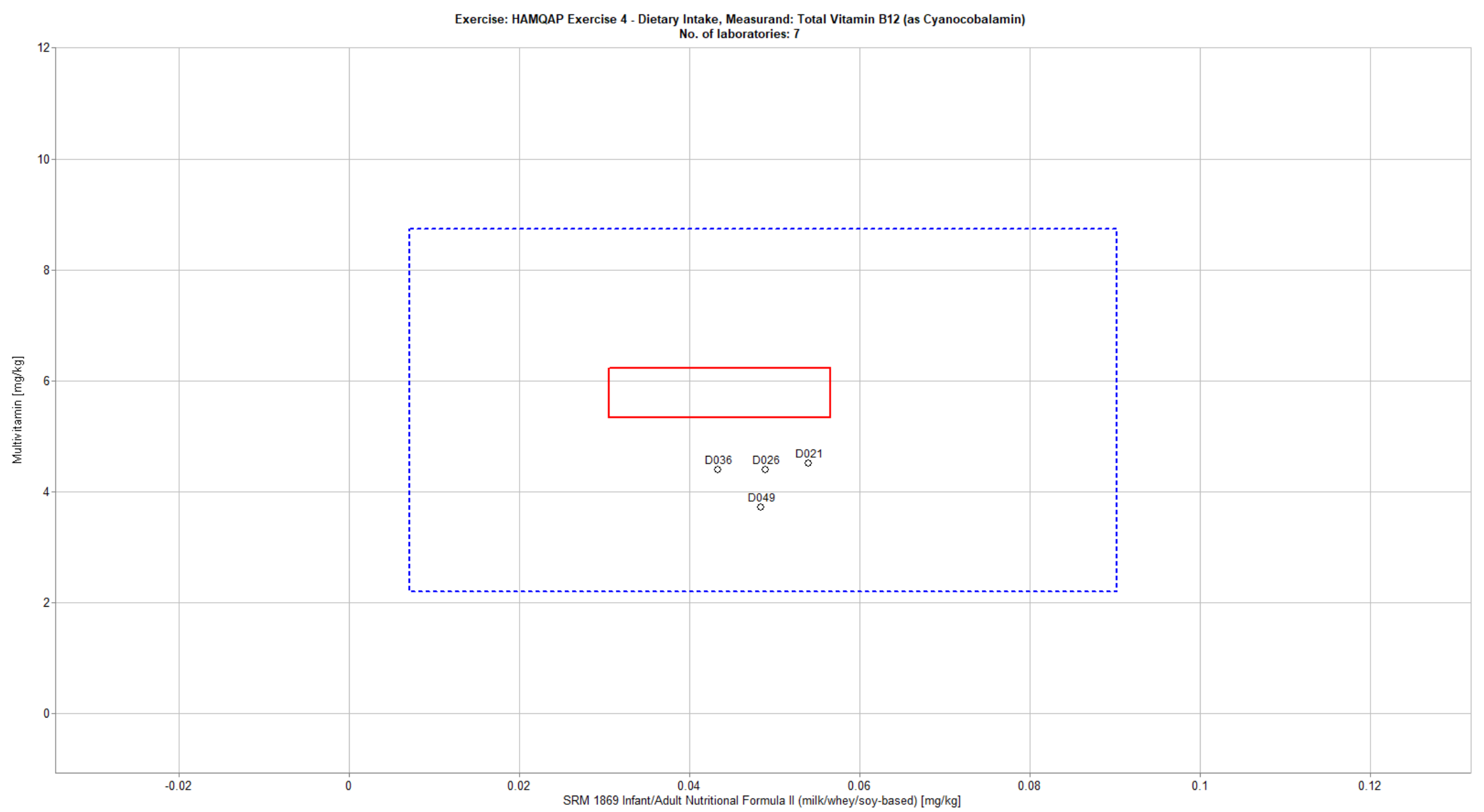

Figure 3-3. Laboratory means for Vitamin $B_{12}$ in SRM 1869 Infant/Adult Nutritional Formula II and Multivitamin (sample/sample comparison view). In this view, the individual laboratory mean for one sample (SRM 1869) is compared to the individual laboratory mean for a second sample (multivitamin). The solid red box represents the NIST range of tolerance for the two samples, SRM 1869 (x-axis) and multivitamin (y-axis), which encompasses the target values bounded by their uncertainties ( $\left.U_{\text {NIST }}\right)$ and represents the range that results in an acceptable $Z_{\mathrm{NIST}}$ score, $\left|Z_{\mathrm{NIST}}\right| \leq 2$. The dotted blue box represents the consensus range of tolerance for SRM 1869 (x-axis) and multivitamin (y-axis), calculated as the values above and below the consensus means that result in an acceptable $Z_{\text {comm }}^{\prime}$ score, $\left|Z_{\text {comm }}^{\prime}\right| \leq 2$. 


\section{SECTION 4: FAT-SOLUBLE VITAMINS (Vitamin $K_{1}$, Vitamin $K_{2}$ )}

\section{Study Overview}

In this study, participants were provided with samples of commercial sauerkraut and multivitamin tablets for dietary intake. Participants were asked to use in-house analytical methods to determine and report the mass fraction $\left(\mathrm{mg} / \mathrm{kg}\right.$ ) of vitamin $\mathrm{K}_{1}$ and vitamin $\mathrm{K}_{2}$ in the two materials. Vitamin $\mathrm{K}$ is a family of fat-soluble vitamins found in some foods and available as a dietary supplement. ${ }^{10}$ The naturally occurring compounds include phylloquinone (vitamin $\mathrm{K}_{1}$ ) and menaquinones (vitamin $\mathrm{K}_{2}$ ). Vitamin $\mathrm{K}_{2}$ compounds are designated as MK-4 through MK-13, based on the length of their side chain, with MK-4, MK-7, and MK-9 being the most well-studied. Most U.S. diets contain an adequate amount of vitamin $\mathrm{K}$, though some analyses of NHANES datasets have raised concerns about average vitamin $\mathrm{K}$ intakes because only about one-third of the U.S. population consumed vitamin $\mathrm{K}$ above the adequate intake (AI) The significance of these findings is unclear because the AI is only an estimate of need, and reports of vitamin $\mathrm{K}$ deficiency in adults are very rare. Vitamin K deficiency has been linked to osteoporosis and coronary heart disease. The population groups most likely to have inadequate vitamin $\mathrm{K}$ are newborns not treated with vitamin $\mathrm{K}$ at birth and people with malabsorption disorders. No adverse effects of excessive vitamin $\mathrm{K}$ intake have been reported, although certain medications can antagonize vitamin $\mathrm{K}$ (notably Warfarin (Coumadin ${ }^{\circledR}$ ) and similar anticoagulants) and or may cause adverse effects on vitamin $\mathrm{K}$ levels (e.g., antibiotics, bile acid sequestrants) have been found.

\section{Dietary Intake Sample Information}

Sauerkraut. Participants were provided with one can containing $14 \mathrm{oz}$ of commercial sauerkraut. Participants were asked to store the unopened can of material at controlled room temperature, $20^{\circ} \mathrm{C}$ to $25^{\circ} \mathrm{C}$, and to prepare three samples and report three values from the single can provided. Before use, participants were instructed to homogenize the contents of the can then mix to ensure homogeneity and to use a sample size of at least $5 \mathrm{~g}$. The approximate analyte levels were not reported to participants prior to the study. Target values for vitamin $\mathrm{K}$ in the sauerkraut have not been determined by NIST.

Multivitamin. Participants were provided with three bottles, each containing 30 multivitamin tablets. Participants were asked to store the material at controlled room temperature, $20{ }^{\circ} \mathrm{C}$ to $25^{\circ} \mathrm{C}$, in the original unopened bottles. Before use, participants were instructed to grind all 30 tablets and mix the resulting powder thoroughly prior to removal of a test portion for analysis, and to use a sample size of at least $1 \mathrm{~g}$ to $1.5 \mathrm{~g}$. After grinding, participants were asked to store the material at $-20{ }^{\circ} \mathrm{C}$. Participants were instructed to prepare one sample and report one value from each bottle provided. Approximate analyte levels were not reported to participants prior to the study. The NIST-determined values for vitamin $\mathrm{K}_{1}$ were assigned using results from the manufacturer of the material. The NIST-determined value and uncertainty for vitamin $\mathrm{K}_{1}$ are provided in the table below on an as-received basis. A target value for vitamin $\mathrm{K}_{2}$ in the multivitamin has not been determined by NIST.

Analyte

Total Vitamin $\mathrm{K}_{1}$
NIST-Determined Value in Multivitamin ( $\mathrm{mg} / \mathrm{kg})$

$$
16.3 \pm 0.4
$$

\footnotetext{
${ }^{10}$ Vitamin K Fact Sheet for Health Professionals. National Institutes of Health Office of Dietary Supplements. https://ods.od.nih.gov/factsheets/vitaminK-HealthProfessional/ (accessed March 2020).
} 


\section{Dietary Intake Study Results}

- Twenty laboratories enrolled in this exercise and received samples to measure vitamin $\mathrm{K}$. The table below summarizes the participation statistics. Some of the reported values were nonquantitative (zero or below LOQ) but are included here in the participation and reporting statistics.

\begin{tabular}{|c|c|c|c|}
\hline \multirow[b]{2}{*}{ Analyte } & \multirow{2}{*}{$\begin{array}{c}\frac{\text { Number of }}{\text { Laboratories }} \\
\text { Requesting Samples }\end{array}$} & \multicolumn{2}{|c|}{$\frac{\text { Number of Laboratories Reporting Result }}{\underline{\text { (Percent Participation) }}}$} \\
\hline & & $\underline{\text { Sauerkraut }}$ & Multivitamin \\
\hline$c i s$-Vitamin $\mathrm{K}_{1}$ & 9 & $0(0 \%)$ & $1(11 \%)$ \\
\hline trans-Vitamin $\mathrm{K}_{1}$ & 9 & $0(0 \%)$ & $2(22 \%)$ \\
\hline Total Vitamin $\mathrm{K}_{1}$ & 20 & $4(20 \%)$ & $11(55 \%)$ \\
\hline Vitamin $\mathrm{K}_{2} \mathrm{MK}-4$ & 16 & $0(0 \%)$ & $1(6 \%)$ \\
\hline Vitamin $\mathrm{K}_{2} \mathrm{MK}-7$ & 16 & $0(0 \%)$ & $3(19 \%)$ \\
\hline Vitamin $\mathrm{K}_{2} \mathrm{MK}-9$ & 8 & $0(0 \%)$ & $0(0 \%)$ \\
\hline
\end{tabular}

- The between-laboratory variabilities for trans-vitamin $\mathrm{K}_{1}$ and total vitamin $\mathrm{K}_{1}$ were acceptable in the multivitamin and high for the sauerkraut (see table below). Between-laboratory variability was not calculated for other vitamin $\mathrm{K}$ forms for which too few quantitative results were reported.

\section{Between-Laboratory Variability (\% RSD)}

$\begin{array}{ccc}\frac{\text { Analyte }}{\text { trans-Vitamin } \mathrm{K}_{1}} & \text { Sauerkraut } & \frac{\text { Multivitamin }}{32 \%} \\ \text { Total Vitamin } \mathrm{K}_{1} & -- & 20 \%\end{array}$

- For the determination of vitamin $\mathrm{K}$ in sauerkraut, two laboratories reported using solvent extraction followed by LC-absorbance, with one laboratory reporting use of LC-fluorescence. One laboratory did not specify any analytical method.

- For the determination of vitamin $\mathrm{K}$ in the multivitamin, most laboratories reported using solvent extraction followed by LC-absorbance, with one laboratory each reporting use of LCfluorescence and LC-MS. One laboratory did not specify any analytical method.

\section{Dietary Intake Technical Recommendations}

The following recommendations are based on results obtained from the participants in this study. In most cases, too few data were reported to allow for meaningful conclusions to be drawn. Figures were chosen to show results according to analytical method.

- Many of the results reported for total vitamin $\mathrm{K}_{1}$ in the multivitamin were within the $95 \%$ consensus range of tolerance and several of these were near the target value.

- Several laboratories reported results significantly higher than the target range, which may be due to improper reporting units or miscalculation of dilution factors. Calculations and reporting units must be verified prior to data submission. 
- When using absorbance as a detection method, compounds that absorb at the same wavelength used for detection of vitamin $\mathrm{K}$ (e.g., other vitamin $\mathrm{K}$ species, matrix components) may cause chromatographic interference and overestimation of the mass fraction of vitamin $\mathrm{K}$ in an unknown sample. All LC separations should be thoroughly evaluated for proper resolution of known or suspected potential interferences.

- For vitamin K compounds, calibrant purity and concentration assignment is best established using spectrophotometric approaches. Improper calibration characterization may lead to biased results.

- Four laboratories reported values for total vitamin $K_{1}$ in the sauerkraut within the $95 \%$ consensus range of tolerance, however this range spanned almost $50 \%$ of the consensus mean. With such low participation and the lack of a reference value, meaningful conclusions cannot be drawn from these results.

- Only a few laboratories reported results for the different forms of vitamin $\mathrm{K}_{1}$ and vitamin $\mathrm{K}_{2}$ in the multivitamin.

- The reported values for cis- and trans- vitamin $\mathrm{K}_{1}$ appear to approximately equal those of the values for total vitamin $\mathrm{K}_{1}$, indicating a possible misidentification of the isomers.

- The type of column and mobile phase play key roles in the separation of vitamin $\mathrm{K}_{1}$ isomers, and the use of a reference material can help establish a method is working properly.

- Some of the values reported for vitamin $\mathrm{K}_{2}$ in the multivitamin were below the LOQ.

- The low participation in this study may indicate a disinterest in or a lack of ability to quantify the individual forms of vitamin $\mathrm{K}$ in multivitamin samples.

- Very few laboratories participated in the sauerkraut portion of the study, which may indicate a disinterest in or a lack of ability to quantify the individual forms of vitamin $\mathrm{K}$ in endogenous food samples.

- The use of appropriate calibration materials and quality assurance samples to establish that a method is in control and being performed correctly may reduce the likelihood of outlying data. Quality assurance samples can be commercially available reference materials (CRMs, SRMs, or RMs) or materials prepared in-house. 
Table 4-1. Individualized data summary table (NIST) for vitamin K in sauerkraut and multivitamin.

\section{National Institute of Standards \& Technology}

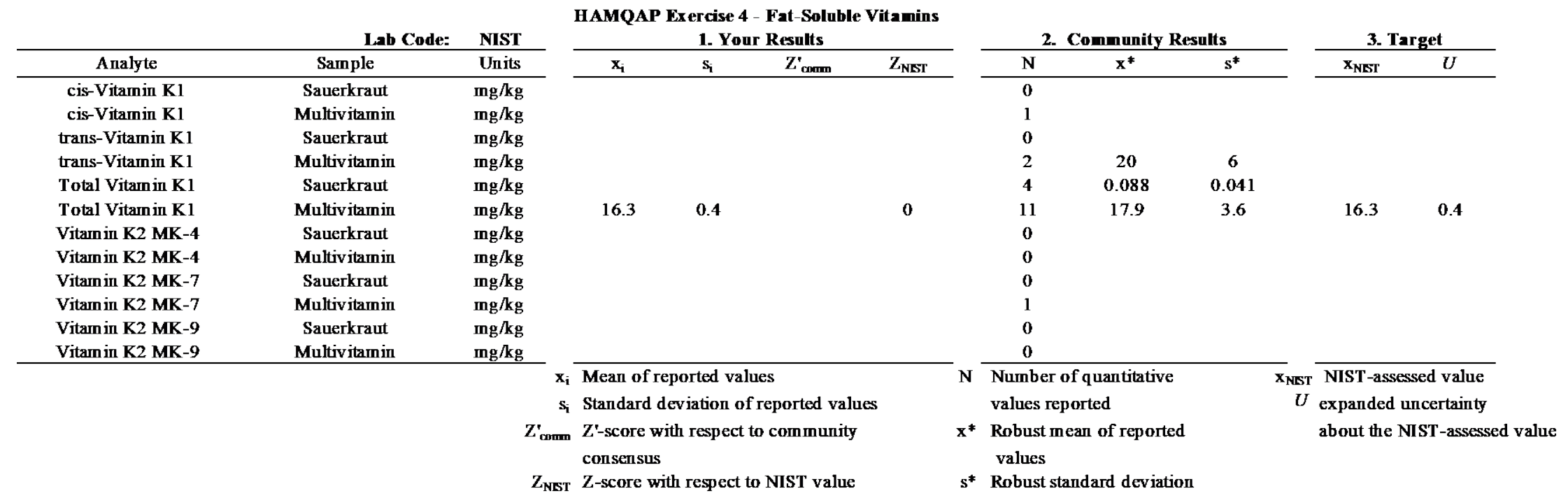


Table 4-2. Data summary table for $c i s$-vitamin $\mathrm{K}_{1}$ in sauerkraut and multivitamin.

\begin{tabular}{|c|c|c|c|c|c|c|c|c|c|c|c|}
\hline & \multirow[b]{3}{*}{ Lab } & \multicolumn{10}{|c|}{ cis-Vitamin $\mathrm{K}_{1}$} \\
\hline & & & & aut & kg) & & & Multi & itamin $(n$ & z/kg) & \\
\hline & & $\mathbf{A}$ & B & $\mathrm{C}$ & Avg & SD & $\mathbf{A}$ & B & C & Avg & SD \\
\hline \multirow{10}{*}{ 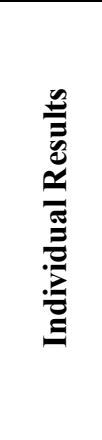 } & Target & & & & & & & & & & \\
\hline & D005 & & & & & & & & & & \\
\hline & D007 & & & & & & & & & & \\
\hline & D009 & & & & & & & & & & \\
\hline & D010 & & & & & & & & & & \\
\hline & D023 & & & & & & & & & & \\
\hline & D034 & & & & & & & & & & \\
\hline & D049 & & & & & & & & & & \\
\hline & D050 & & & & & & & & & & \\
\hline & D055 & & & & & & 15.9649 & 16.6343 & 16.4991 & 16.37 & 0.35 \\
\hline 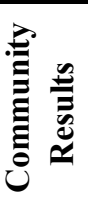 & & \multicolumn{5}{|c|}{$\begin{array}{l}\text { Consensus Mean } \\
\text { Consensus Standard Deviation } \\
\text { Maximum } \\
\text { Minimum } \\
\mathrm{N}\end{array}$} & \multicolumn{5}{|c|}{$\begin{array}{l}\text { Consensus Mean } \\
\text { Consensus Standard Deviation } \\
\text { Maximum } \\
\text { Minimum } \\
\text { N }\end{array}$} \\
\hline
\end{tabular}

Table 4-3. Data summary table for trans-vitamin $\mathrm{K}_{1}$ in sauerkraut and multivitamin.

\begin{tabular}{|c|c|c|c|c|c|c|c|c|c|c|c|}
\hline & \multirow[b]{3}{*}{ Lab } & \multicolumn{10}{|c|}{ trans-Vitamin $\mathrm{K}_{1}$} \\
\hline & & & & aut ( & & & & Mult & itamin (n & (kg) & \\
\hline & & $\mathbf{A}$ & B & $\mathrm{C}$ & Avg & SD & $\mathbf{A}$ & B & $\mathbf{C}$ & Avg & SD \\
\hline \multirow{10}{*}{ 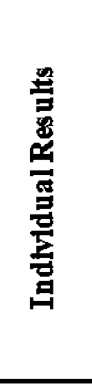 } & Target & & & & & & & & & & \\
\hline & D005 & & & & & & & & & & \\
\hline & D007 & & & & & & & & & & \\
\hline & D009 & & & & & & & & & & \\
\hline & D010 & & & & & & & & & & \\
\hline & D023 & & & & & & 20.3 & 21.14 & 20.86 & 20.77 & 0.43 \\
\hline & D034 & & & & & & & & & & \\
\hline & D049 & & & & & & & & & & \\
\hline & D050 & & & & & & & & & & \\
\hline & $\mathrm{D} 055$ & & & & & & 16.4449 & 16.6096 & 16.5905 & 16.55 & 0.09 \\
\hline \multirow{5}{*}{ 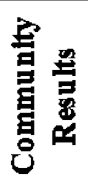 } & & \multirow{5}{*}{\multicolumn{3}{|c|}{$\begin{array}{l}\text { Consensus Mean } \\
\text { Consensus Standard Deviation } \\
\text { Maximum } \\
\text { Minimum } \\
\text { N }\end{array}$}} & & & \multirow{5}{*}{\multicolumn{3}{|c|}{$\begin{array}{l}\text { Consensus Mean } \\
\text { Consensus Standard Deviation } \\
\text { Maximum } \\
\text { Minimum } \\
\text { N }\end{array}$}} & 18.66 & \\
\hline & & & & & & & & & & 5.99 & \\
\hline & & & & & & & & & & 20.77 & \\
\hline & & & & & & & & & & 16.55 & \\
\hline & & & & & 0 & & & & & & \\
\hline
\end{tabular}


Table 4-4. Data summary table for total vitamin $K_{1}$ in sauerkraut and multivitamin. Data points highlighted in red have been flagged as potential outliers (e.g., Grubb and/or Cochran) by the NIST software package.

\begin{tabular}{|c|c|c|c|c|c|c|c|c|c|c|c|}
\hline & \multirow[b]{3}{*}{ Lab } & \multicolumn{10}{|c|}{ Total Vitamin $K_{1}$} \\
\hline & & \multicolumn{5}{|c|}{ Sau erkraut (mg/kg) } & \multicolumn{5}{|c|}{ Multivitamin (mg/kg) } \\
\hline & & $\mathbf{A}$ & $\mathbf{B}$ & $\mathrm{C}$ & Avg & SD & $\mathbf{A}$ & $\bar{B}$ & $\mathrm{C}$ & Avg & SD \\
\hline \multirow{21}{*}{ 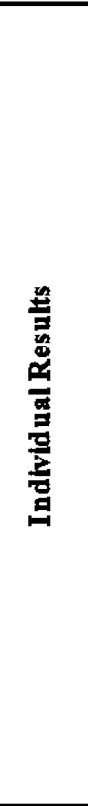 } & Target & & & & & & & & & 16.30 & 0.40 \\
\hline & D001 & & & & & & 52.8 & 55.2 & 54.7 & 54.2 & 1.3 \\
\hline & $\mathrm{D} 004$ & & & & & & 1238.14 & 1294.03 & 1252.43 & 1261.5 & 29.0 \\
\hline & D005 & & & & & & 10.91 & 11.13 & 11.49 & 11.2 & 0.3 \\
\hline & D007 & & & & & & & & & & \\
\hline & D009 & & & & & & 10.68 & 10.33 & 10.52 & 10.5 & 0.2 \\
\hline & D010 & & & & & & 11.1 & 11.9 & 11.3 & 11.4 & 0.4 \\
\hline & D011 & & & & & & & & & & \\
\hline & $\mathrm{D} 017$ & & & & & & & & & & \\
\hline & D019 & & & & & & 20.9671 & 22.2324 & 22.3102 & 21.8 & 0.8 \\
\hline & D021 & & & & & & 28 & 27.3 & 27.5 & 27.6 & 0.4 \\
\hline & D023 & 0.04 & 0.04 & 0.04 & 0.040 & 0.000 & & & & & \\
\hline & $\mathrm{D} 026$ & & & & & & & & & & \\
\hline & D034 & & & & & & & & & & \\
\hline & D036 & 0.08 & 0.09 & 0.09 & 0.087 & 0.006 & & & & & \\
\hline & D042 & & & & & & & & & & \\
\hline & D045 & & & & & & & & & & \\
\hline & D048 & & & & & & 131 & 147 & 138 & 138.7 & 8.0 \\
\hline & D049 & 0.165 & 0.135 & 0.141 & 0.147 & 0.016 & 15.7 & 15.9 & 15.7 & 15.8 & 0.1 \\
\hline & D050 & & & & & & 11.21 & 11.14 & 11.17 & 11.2 & 0.0 \\
\hline & D055 & 0.08 & 0.079 & 0.07 & 0.076 & 0.006 & 16.2049 & 16.622 & 16.5448 & 16.5 & 0.2 \\
\hline 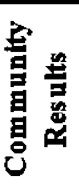 & & $\begin{array}{l}\text { Consens } \\
\text { Consens } \\
\text { Maximu } \\
\text { Minimu } \\
\text { N }\end{array}$ & $\begin{array}{l}\text { Mean } \\
\text { Standarc }\end{array}$ & eviation & $\begin{array}{c}0.088 \\
0.041 \\
0.147 \\
0.040 \\
4\end{array}$ & & $\begin{array}{l}\text { Consens } \\
\text { Consens } \\
\text { Maximur } \\
\text { Minimun } \\
\text { N }\end{array}$ & $\begin{array}{l}\text { Mean } \\
\text { Standard }\end{array}$ & Deviation & $\begin{array}{c}17.9 \\
3.6 \\
1261.5 \\
10.5 \\
11\end{array}$ & \\
\hline
\end{tabular}




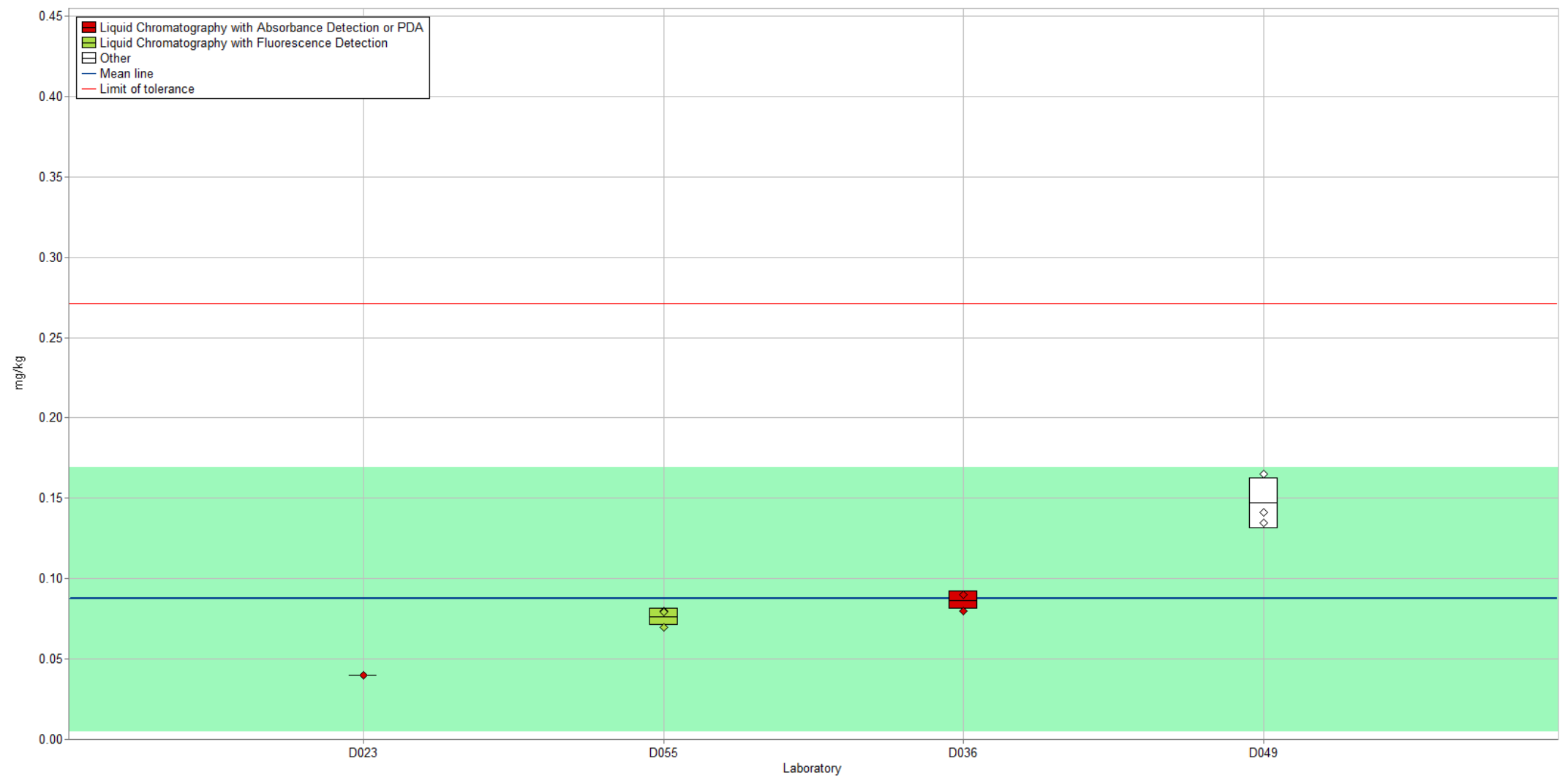

Figure 4-1. Total Vitamin $K_{1}$ in Sauerkraut (data summary view - analytical method). In this view, individual laboratory data are plotted (diamonds) with the individual laboratory standard deviation (rectangle). The color of the data point represents the analytical detection method employed. The solid blue line represents the consensus mean, and the green shaded region represents the $95 \%$ confidence interval for the consensus mean. The solid red line represents the upper consensus range of tolerance, calculated as the values above the consensus mean that result in an acceptable $Z_{\text {comm }}^{\prime}$ score, $\left|Z_{\text {comm }}^{\prime}\right| \leq 2$, with the lower limit set at zero. A NIST value has not been determined in this material. 
Exercise HAMQAP Exercise 4 - Dietary Intake ample: : Multivitamin $\mathrm{k} 1$

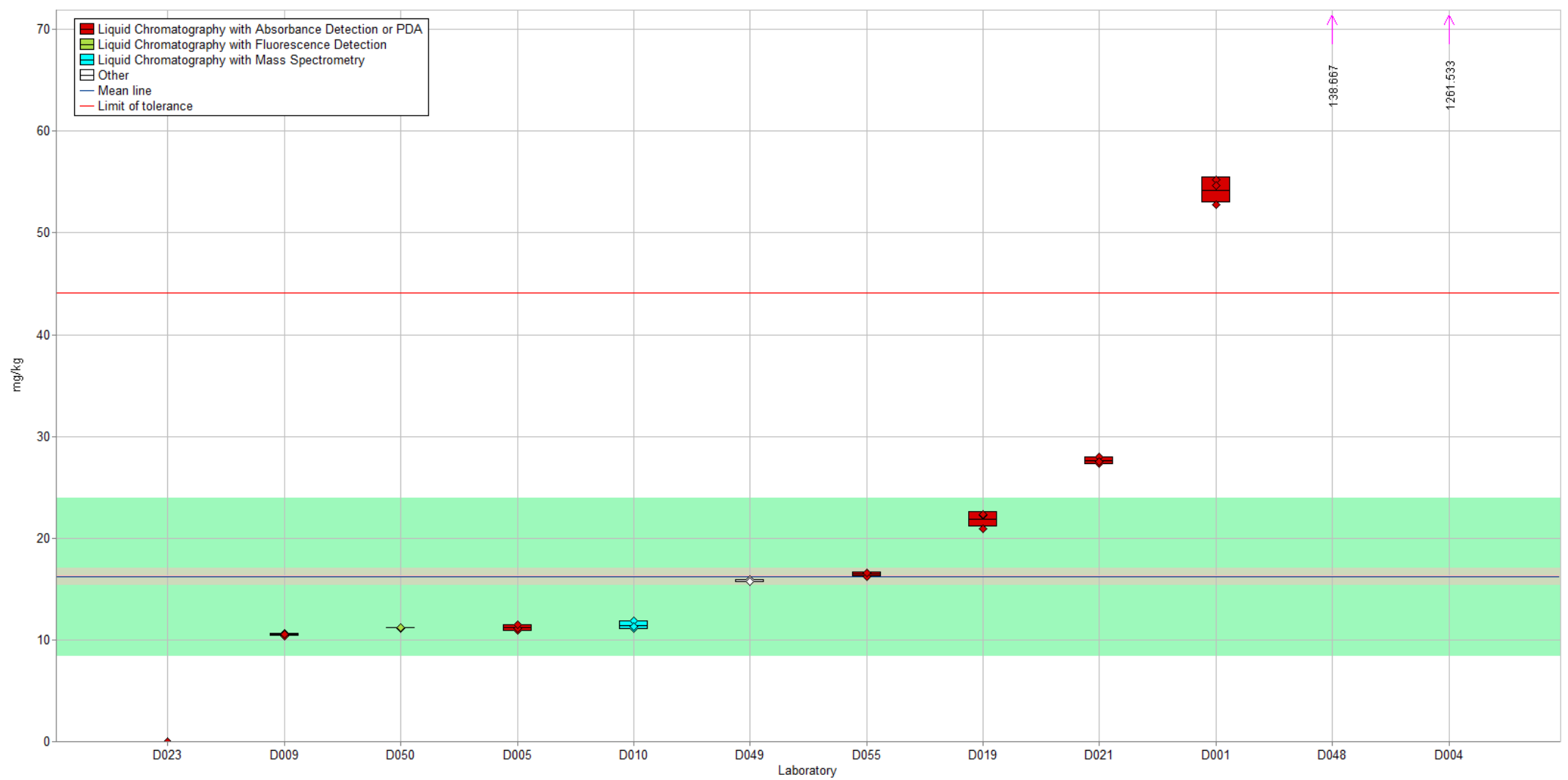

Figure 4-2. Total Vitamin $K_{1}$ in Multivitamin (data summary view - analytical method). In this view, individual laboratory data are plotted (diamonds) with the individual laboratory standard deviation (rectangle). The color of the data point represents the sample preparation method employed. The solid blue line represents the consensus mean, and the green shaded region represents the $95 \%$ confidence interval for the consensus mean. The solid red line represents the upper consensus range of tolerance, calculated as the values above the consensus mean that result in an acceptable $Z_{\text {comm }}^{\prime}$ score, $\left|Z_{\text {comm }}^{\prime}\right| \leq 2$, with the lower limit set at zero. The red shaded region represents the NIST range of tolerance, which encompasses the target value bounded by its uncertainty ( $\left.U_{\mathrm{NIST}}\right)$ and represents the range that results in an acceptable $Z_{\mathrm{NIST}}$ score, $\left|Z_{\mathrm{NIST}}\right| \leq 2$. 


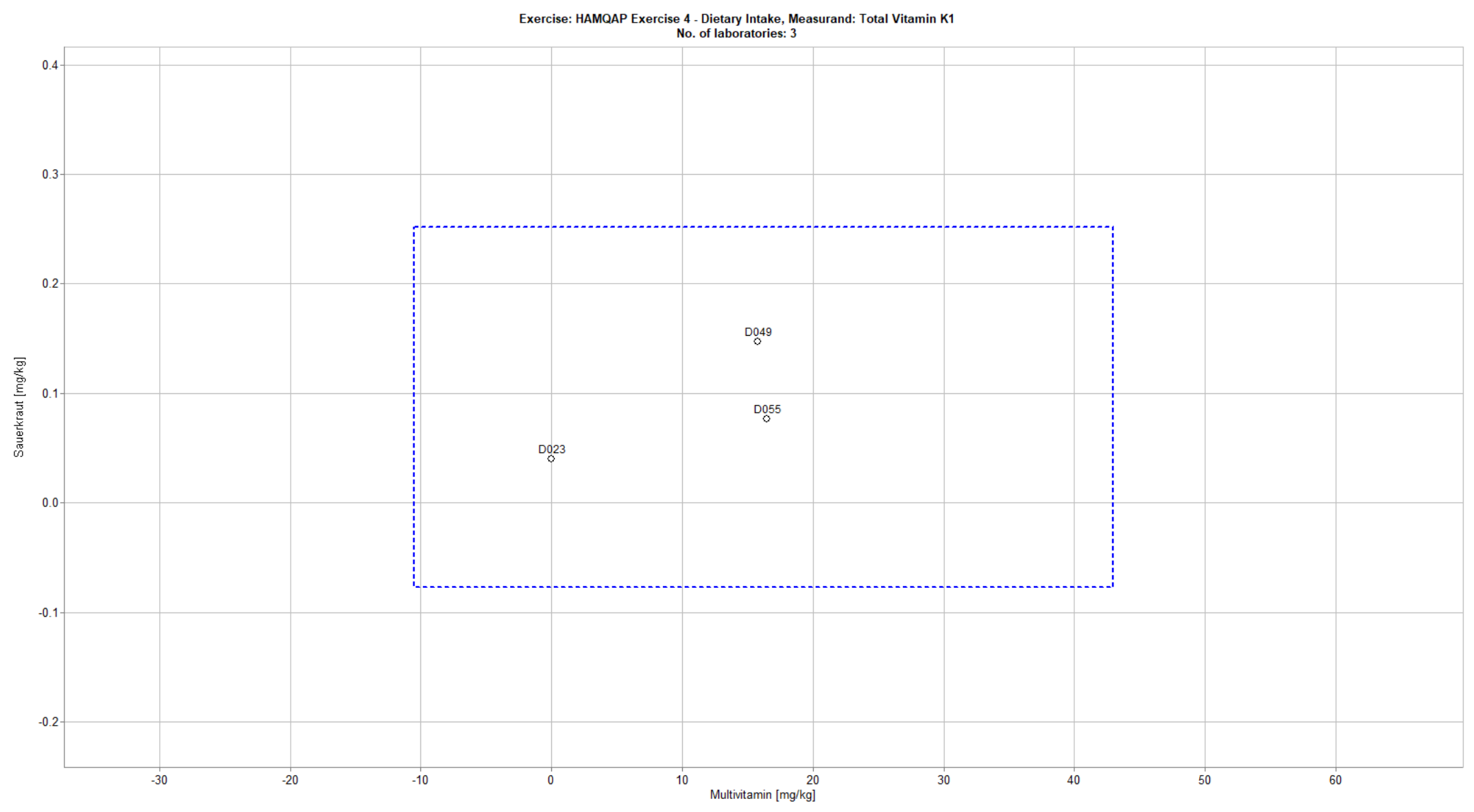

Figure 4-3. Laboratory means for total vitamin $\mathrm{K}_{1}$ in Sauerkraut and Multivitamin (sample/sample comparison view). In this view, the individual laboratory mean for one sample (sauerkraut) is compared to the mean for a second sample (multivitamin). The dotted blue box represents the consensus range of tolerance for sauerkraut (x-axis) and multivitamin (y-axis), calculated as the values above and below the consensus means that result in an acceptable $Z_{\text {comm }}^{\prime}$ score, $\left|Z_{\text {comm }}^{\prime}\right| \leq 2$. 
Table 4-5. Data summary table for vitamin $\mathrm{K}_{2}$ MK-4 in sauerkraut and multivitamin.

\begin{tabular}{|c|c|c|c|c|c|c|c|c|c|c|c|}
\hline & \multirow[b]{3}{*}{ Lab } & \multicolumn{10}{|c|}{ Vitamin $K_{2}$ MK-4 } \\
\hline & & \multicolumn{5}{|c|}{ Sauerkraut (mg/kg) } & \multicolumn{5}{|c|}{ Multivitamin (mg/kg) } \\
\hline & & $\mathbf{A}$ & B & $\mathrm{C}$ & Avg & SD & $\mathbf{A}$ & $\mathbf{B}$ & $\mathrm{C}$ & Avg & SD \\
\hline \multirow{17}{*}{ 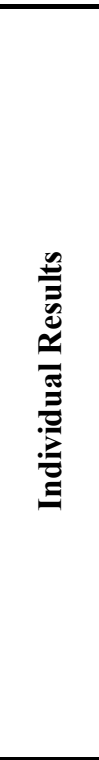 } & Target & & & & & & & & & & \\
\hline & D001 & & & & & & & & & & \\
\hline & D005 & & & & & & & & & & \\
\hline & D007 & & & & & & & & & & \\
\hline & D009 & & & & & & & & & & \\
\hline & D010 & & & & & & $<0.18$ & $<0.188$ & $<0.188$ & & \\
\hline & D011 & & & & & & & & & & \\
\hline & D019 & & & & & & & & & & \\
\hline & D021 & & & & & & & & & & \\
\hline & D023 & & & & & & & & & & \\
\hline & D026 & & & & & & & & & & \\
\hline & D034 & & & & & & & & & & \\
\hline & D042 & & & & & & & & & & \\
\hline & D045 & & & & & & & & & & \\
\hline & D049 & & & & & & & & & & \\
\hline & D050 & & & & & & & & & & \\
\hline & D055 & & & & & & & & & & \\
\hline 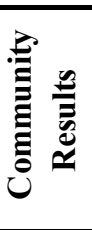 & & $\begin{array}{l}\text { Conse } \\
\text { Conse } \\
\text { Maxin } \\
\text { Minim } \\
\mathrm{N}\end{array}$ & $\begin{array}{l}\text { lea } \\
\tan \end{array}$ & evia & 0 & & $\begin{array}{l}\text { Conse } \\
\text { Conse } \\
\text { Maxin } \\
\text { Minim } \\
\mathrm{N}\end{array}$ & $\begin{array}{l}\text { s Mean } \\
\text { s Standar }\end{array}$ & Deviation & 0 & \\
\hline
\end{tabular}


Table 4-6. Data summary table for vitamin $\mathrm{K}_{2} \mathrm{MK}-7$ in sauerkraut and multivitamin.

\begin{tabular}{|c|c|c|c|c|c|c|c|c|c|c|c|}
\hline & \multirow[b]{3}{*}{ Lab } & \multicolumn{10}{|c|}{ Vitamin $K_{2}$ MK-7 } \\
\hline & & \multicolumn{5}{|c|}{ Sauerkraut (mg/kg) } & \multicolumn{5}{|c|}{ Multivitamin (mg/kg) } \\
\hline & & $\mathbf{A}$ & B & C & Avg & SD & $\mathbf{A}$ & B & $\mathrm{C}$ & Avg & SD \\
\hline \multirow{17}{*}{ 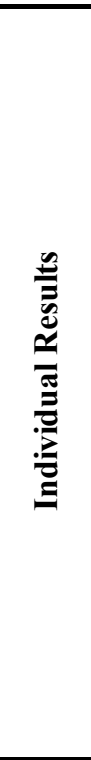 } & Target & & & & & & & & & & \\
\hline & D004 & & & & & & 1187.29 & 1167.47 & 1139.16 & 1165 & 24 \\
\hline & D005 & & & & & & & & & & \\
\hline & D007 & & & & & & & & & & \\
\hline & D009 & & & & & & & & & & \\
\hline & D010 & & & & & & $<0.192$ & $<0.192$ & $<0.192$ & & \\
\hline & D011 & & & & & & & & & & \\
\hline & D019 & & & & & & & & & & \\
\hline & D021 & & & & & & $<3.89$ & $<3.89$ & $<3.89$ & & \\
\hline & D023 & & & & & & & & & & \\
\hline & D034 & & & & & & & & & & \\
\hline & D042 & & & & & & & & & & \\
\hline & D045 & & & & & & & & & & \\
\hline & D048 & & & & & & & & & & \\
\hline & D049 & & & & & & & & & & \\
\hline & D050 & & & & & & & & & & \\
\hline & D055 & & & & & & & & & & \\
\hline 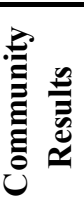 & & $\begin{array}{l}\text { Conse } \\
\text { Conse } \\
\text { Maxin } \\
\text { Minim }\end{array}$ & $\begin{array}{l}\text { lea } \\
\tan \end{array}$ & evia & & & $\begin{array}{l}\text { Consensus } \\
\text { Consensus } \\
\text { Maximum } \\
\text { Minimum }\end{array}$ & $\begin{array}{l}\text { Mean } \\
\text { Standard }\end{array}$ & Deviation & $\begin{array}{l}1165 \\
1165\end{array}$ & \\
\hline & & $\mathrm{N}$ & & & 0 & & $\mathrm{~N}$ & & & 1 & \\
\hline
\end{tabular}




\section{SECTION 5: FATTY ACIDS (Omega-3 and Omega-6 Fatty Acids)}

\section{Study Overview}

In this study, participants were provided with samples of SRM 3275 Omega-3 and Omega-6 Fatty Acids in Fish Oil Level 1 and Level 3 for dietary intake, and with samples of SRM 2378 Fatty Acids in Frozen Human Serum Level 1 and Level 2 for human metabolism. Participants were asked to use in-house analytical methods to determine the mass fraction $(\mathrm{mg} / \mathrm{g})$ of omega-3 and omega-6 in each matrix. Omega-3 fatty acids are important components of the phospholipids that form the structures of cell membranes. ${ }^{11}$ In addition, omega- 3 and omega- 6 fatty acids provide energy for the body and are used to form eicosanoids, which are mediators of inflammation, vasoconstriction, and platelet aggregation. Some researchers propose that the relative intakes of omega-3s and omega-6s may have important implications for the pathogenesis of chronic diseases such as cardiovascular disease and cancer, but an optimal ratio has not yet been defined. Scientific research has mostly focused on three omega-3 fatty acids, $\alpha$-linolenic acid (ALA), eicosapentaenoic acid (EPA), and docosahexaenoic acid (DHA), and two omega-6 fatty acids, linoleic acid and arachidonic acid (ARA). Dietary sources of EPA and DHA include fish and fish oils, as fatty acids originally synthesized by microalgae further down the food chain accumulate in fish tissues. ALA and other omega- 6 fatty acids can be found in plant sources such as plant oils, chia seeds, and walnuts. Omega-3 and omega- 6 fatty acid health status can be evaluated by measuring individual components in plasma or serum phospholipids, but values can vary substantially based on an individual's most recent intake and as such do not reflect long-term dietary consumption. Understanding intake of omega-3 and omega-6 fatty acids and their impact on inflammation and disease can advance clinical research that investigates how manipulating the omega- 6 to omega-3 ratio may yield positive health outcomes.

\section{Dietary Intake Sample Information}

Fish Oil $A$ and B. Participants were provided with three ampoules of SRM 3275 Omega-3 and Omega-6 Fatty Acids in Fish Oil Level 1 and three vials of SRM 3275 Omega-3 and Omega-6 Fatty Acids in Fish Oil Level 3, each containing $1.2 \mathrm{~mL}$ of fish oil. Level 1 is a concentrate high in DHA, and Level 3 is a concentrate containing $60 \%$ long-chain omega-3 fatty acids. Participants were asked to store the materials under refrigeration, $2{ }^{\circ} \mathrm{C}$ to $8{ }^{\circ} \mathrm{C}$, in the original unopened ampoules and to prepare one sample and report one value from each ampoule provided. Before use, participants were instructed to thoroughly mix the contents of the ampoule prior to removal of a test portion for analysis, and to use a sample size of at least $0.5 \mathrm{~g}$. The approximate analyte levels were not reported to participants prior to the study. Certified values for linoleic acid and

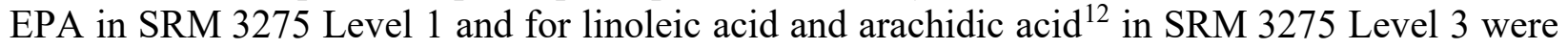
assigned using results from NIST by GC-FID and GC-MS. Reference values for $\alpha$-linolenic acid and DHA in SRM 3275 Level 1 and for $\alpha$-linolenic acid, EPA, and DHA in SRM 3275 Level 3 were assigned using results from NIST by GC-FID. A reference value for arachidic acid ${ }^{12}$ in SRM 3275 Level 1 was assigned using results from NIST by GC-MS. The NIST-determined values and uncertainties for omega-3 and omega-6 fatty acids in SRM 3275 are provided in the

\footnotetext{
${ }^{11}$ Omega-3 Fatty Acids Fact Sheet for Health Professionals. National Institutes of Health Office of Dietary Supplements. https:/ods.od.nih.gov/factsheets/Omega3FattyAcids-HealthProfessional/ (accessed March 2020).

12 Due to an error in the NIST data collection system, arachidic acid data was requested instead of arachidonic acid.
} 
table below, reported both as the fatty acid methyl esters (FAMEs) as listed on the Certificate of Analysis and as the free fatty acids (FFAs), using standard molecular weight conversion factors. ${ }^{13}$

NIST-Determined Mass Fractions (mg/g)

$\underline{\text { SRM 3275-1 }}$

\begin{tabular}{|c|c|c|c|c|}
\hline Analyte & (FAMEs) & (FFAs) & (FAMEs) & (FFAs) \\
\hline$\alpha$-Linolenic Acid & $1.21 \pm 0.05$ & $1.15 \pm 0.05$ & $6.61 \pm 0.31$ & $6.29 \pm 0.30$ \\
\hline Linoleic Acid & $2.31 \pm 0.19$ & $2.20 \pm 0.18$ & $13.49 \pm 0.45$ & $12.85 \pm 0.43$ \\
\hline Arachidic Acid ${ }^{12}$ & $1.910 \pm 0.071$ & $1.828 \pm 0.068$ & $1.14 \pm 0.26$ & $1.09 \pm 0.2$ \\
\hline EPA & \pm 12 & \pm 11 & \pm 9 & 153 \\
\hline DHA & \pm 15 & \pm 14 & \pm 5 & 100 \\
\hline
\end{tabular}

Dietary Intake Study Results

- Twenty-two laboratories enrolled in this exercise and received samples to measure some or all of the fatty acids in fish oils. Nine to 10 laboratories reported results for each analyte, resulting in $41 \%$ to $45 \%$ participation. Participation statistics for each analyte are described in more detail below.

\begin{tabular}{|c|c|c|c|}
\hline & $\frac{\text { Number of }}{\text { Laboratories }}$ & \multicolumn{2}{|c|}{$\frac{\text { Number of Laboratories Reporting Results }}{\text { (Percent Participation) }}$} \\
\hline Analyte & $\frac{\text { Requesting }}{\underline{\text { Samples }}}$ & SRM 3275 Level 1 & SRM 3275 Level 3 \\
\hline$\alpha$-Linolenic Acid & 22 & $9(41 \%)$ & $9(41 \%)$ \\
\hline Linoleic Acid & 22 & $10(45 \%)$ & $10(45 \%)$ \\
\hline Arachidic Acid & 21 & $9(43 \%)$ & $9(43 \%)$ \\
\hline EPA & 22 & $10(45 \%)$ & $10(45 \%)$ \\
\hline DHA & 22 & $10(45 \%)$ & $10(45 \%)$ \\
\hline
\end{tabular}

- The consensus ranges for all fatty acids overlapped the target ranges, except for linoleic acid in SRM 3275 Level 3, where the consensus range was almost completely below the target range (Figure 5-5), and for arachidic acid in both samples, where the consensus ranges were completely above the target ranges (Figures 5-7 and 5-8).

- The between-laboratory variabilities were excellent for all analytes in both matrices, at $10 \%$ or lower relative standard deviation. Variabilities for each analyte/sample pair are reported in the table below.

\footnotetext{
${ }^{13}$ DeVries, J.W., Kjos, L., Groff, L., Martin, B., Cernohous, K., Patel, H., Payne, H., Leichtweis, H., Shay, M., and Newcomer, L. (1999) Studies in Improvement of Official Method 996.06, J. AOAC Int. 82, 1146-1155.
} 


\begin{tabular}{ccc} 
& \multicolumn{2}{c}{ Between-Laboratory Variability (RSD) } \\
Analyte & SRM 3275 Level 1 & SRM 3275 Level 3 \\
\cline { 2 - 3 }$\alpha$-Linolenic Acid & $6 \%$ & $4 \%$ \\
Linoleic Acid & $5 \%$ & $4 \%$ \\
Arachidic Acid & $6 \%$ & $10 \%$ \\
EPA & $2 \%$ & $2 \%$ \\
DHA & $2 \%$ & $2 \%$
\end{tabular}

- Laboratories reported using derivatization to fatty acid methyl esters or acid hydrolysis as the sample preparation method. Some laboratories did not report a sample preparation method.

- Laboratories reported using GC-FID or GC (no detection method specified) as their analytical method for determination of fatty acids in these samples.

Dietary Intake Technical Recommendations

The following recommendations are based on results obtained from the participants in this study.

- The determination of fatty acids in fish oils does not appear to be a challenge for most laboratories. However, laboratories should be aware of the level of sample preparation required and beware of sample over-processing (e.g., unneeded extraction steps) that may introduce atypical errors such as losses or interferences.

- Arachidic acid may have been problematic for some laboratories as an atypical analyte. The upward trend seen among data points in Figure 5-9 may indicate a calibration error.

- No laboratories consistently reported high or low results with respect to the consensus or target ranges, indicating analyte-specific challenges such as calibration errors or interferences.

- A linear calibration curve which surrounds the expected sample concentration values should be used for calculations. This curve should include both the lowest and highest expected concentration values of the sample solutions. Extrapolation of results beyond calibration curves may result in incorrect values.

- Laboratories reporting results flagged as outliers should check for calculation errors. One example is to confirm that factors for all dilutions have been properly tabulated.

- The use of appropriate calibration materials and quality assurance samples to establish that a method is in control and performing correctly may reduce the likelihood of outlying data. Quality assurance samples can be commercially available reference materials (CRMs, SRMs, or RMs) or materials prepared in-house. 
Table 5-1. Individualized data summary table (NIST) for fatty acids in fish oils.

\section{National Institute of Standards \& Technology}

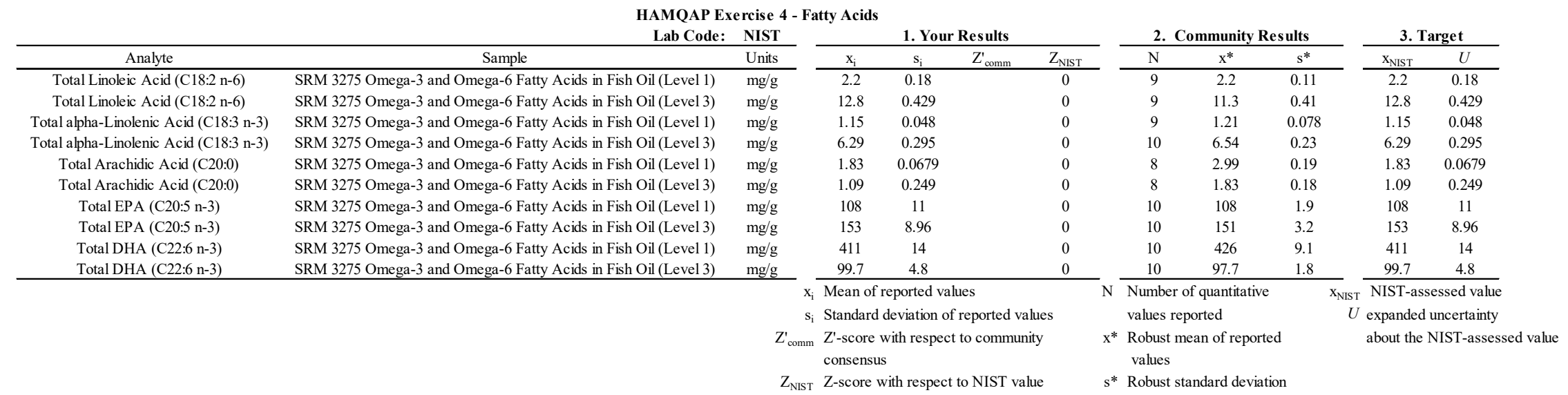


Table 5-2. Data summary table for total $\alpha$-linolenic acid in fish oil. Data points highlighted in red have been flagged as potential outliers (e.g., Grubb and/or Cochran) by the NIST software package.

\begin{tabular}{|c|c|c|c|c|c|c|c|c|c|c|c|}
\hline & & \multicolumn{10}{|c|}{ Total alpha-Linolenic Acid (C18:3 n-3) } \\
\hline & & \multicolumn{5}{|c|}{$\begin{array}{c}\text { SRM } 3275 \text { Omega-3 and Omega-6 Fatty Acids in } \\
\text { Fish Oil (Level 1) (mg/g) }\end{array}$} & \multicolumn{5}{|c|}{$\begin{array}{c}\text { SRM } 3275 \text { Omega-3 and Omega-6 Fatty Acids in } \\
\text { Fish Oil (Level 3) (mg/g) }\end{array}$} \\
\hline & Lab & $\mathbf{A}$ & $\mathbf{B}$ & $\mathbf{C}$ & Avg & SD & $\mathbf{A}$ & $\mathbf{B}$ & $\mathbf{C}$ & Avg & SD \\
\hline \multirow{23}{*}{ 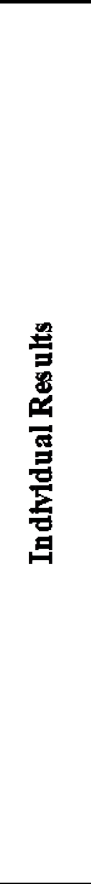 } & Target & & & & 1.152 & 0.048 & & & & 6.29 & 0.30 \\
\hline & D001 & & & & & & & & & & \\
\hline & D003 & & & & & & & & & & \\
\hline & D004 & 1.25 & 1.25 & 1.25 & 1.250 & 0.000 & 6.65 & 6.58 & 6.55 & 6.59 & 0.05 \\
\hline & D005 & 1.11 & 1.09 & 1.1 & 1.100 & 0.010 & 6.54 & 6.49 & 6.42 & 6.48 & 0.06 \\
\hline & D006 & & & & & & & & & & \\
\hline & D007 & & & & & & & & & & \\
\hline & D008 & $<2.00$ & $<2.00$ & $<2.00$ & & & 9.21 & 9.13 & 8.83 & 9.06 & 0.20 \\
\hline & D010 & & & & & & & & & & \\
\hline & D016 & 1.07576 & 1.08528 & 1.10432 & 1.088 & 0.015 & 6.35936 & 6.3308 & 6.43552 & 6.38 & 0.05 \\
\hline & D018 & & & & & & & & & & \\
\hline & D023 & 1 & 1 & 1 & 1.000 & 0.000 & 6.25 & 6.75 & 6.75 & 6.58 & 0.29 \\
\hline & D029 & & & & & & & & & & \\
\hline & D034 & & & & & & & & & & \\
\hline & D036 & 1.37 & 1.35 & 1.37 & 1.363 & 0.012 & 8.39 & 8.56 & 8.56 & 8.50 & 0.10 \\
\hline & D037 & & & & & & & & & & \\
\hline & D039 & & & & & & & & & & \\
\hline & D040 & & & & & & & & & & \\
\hline & D042 & 1.34 & 1.77 & 1.26 & 1.457 & 0.274 & 6 & 5.88 & 5.14 & 5.67 & 0.47 \\
\hline & D044 & 0.945 & 0.893 & 0.935 & 0.924 & 0.028 & 3.993 & 4.245 & 3.946 & 4.06 & 0.16 \\
\hline & D049 & 1.15 & 1.22 & 1.24 & 1.203 & 0.047 & 6.74 & 6.76 & 6.74 & 6.75 & 0.01 \\
\hline & D050 & 1.77 & 1.3 & 1.35 & 1.473 & 0.258 & 6.61 & 6.14 & 6.02 & 6.26 & 0.31 \\
\hline & D055 & & & & & & & & & & \\
\hline \multirow{5}{*}{ 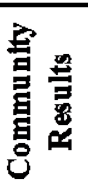 } & & \multirow{5}{*}{\multicolumn{3}{|c|}{$\begin{array}{l}\text { Consensus Mean } \\
\text { Consensus Standard Deviation } \\
\text { Maximum } \\
\text { Minimum } \\
\text { N }\end{array}$}} & 1.207 & & \multirow{5}{*}{\multicolumn{3}{|c|}{$\begin{array}{l}\text { Consensus Mean } \\
\text { Consensus Stand } \\
\text { Maximum } \\
\text { Minimum } \\
\text { N }\end{array}$}} & 6.54 & \\
\hline & & & & & 0.078 & & & & & 0.23 & \\
\hline & & & & & 1.473 & & & & & 9.06 & \\
\hline & & & & & 0.924 & & & & & 4.06 & \\
\hline & & & & & 9 & & & & & 10 & \\
\hline
\end{tabular}


Exercise HAMQAP Exercise 4 - Dietary Intake
Sample: SRM 3275 Omega-3 and Omega-6 Fatty Acids in Fish Oil (Level 1)

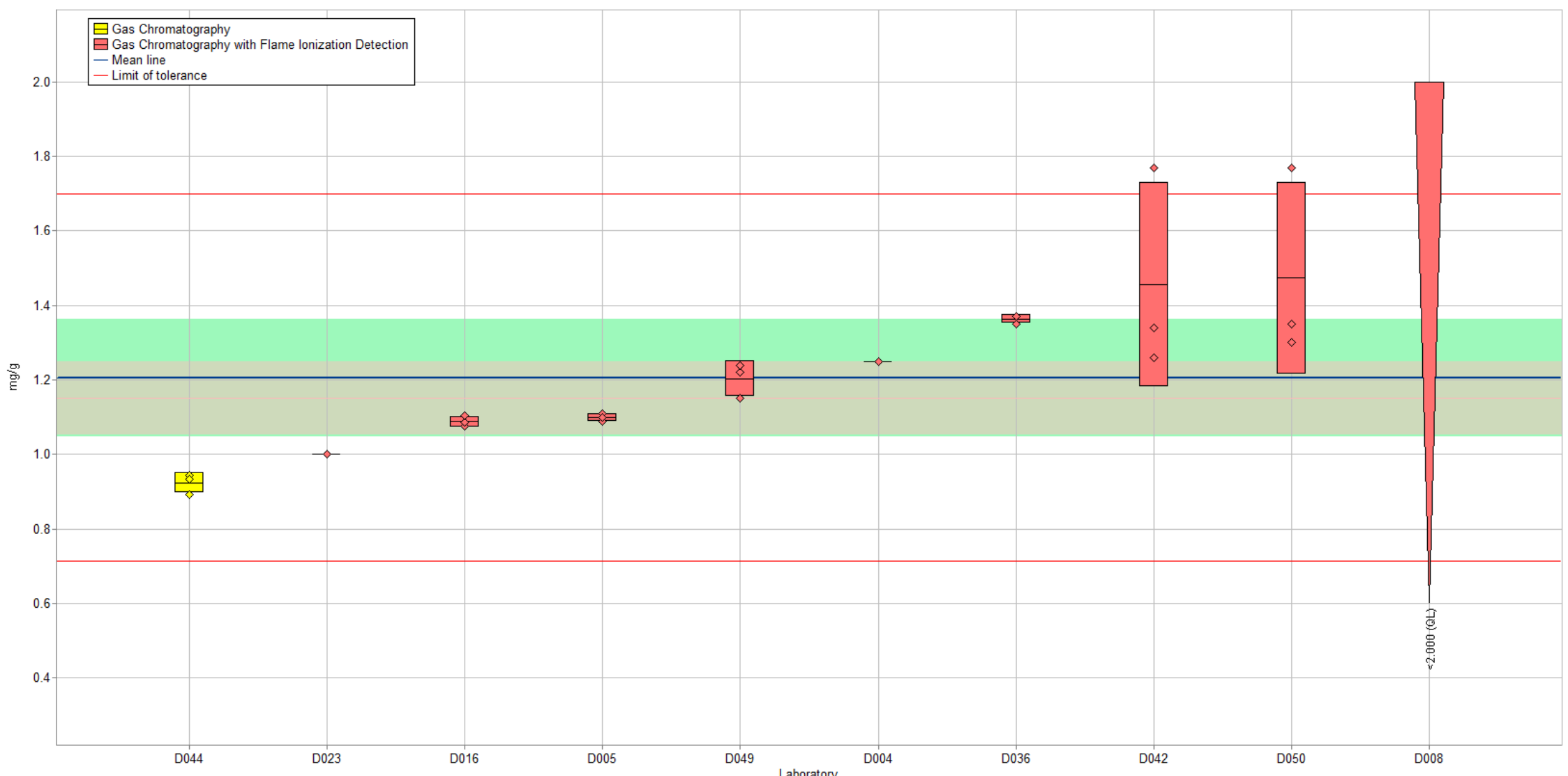

Figure 5-1. Total $\alpha$-linolenic acid in SRM 3275 Omega-3 and Omega-6 Fatty Acids in Fish Oil (Level 1) (data summary view analytical method). In this view, individual laboratory data are plotted (diamonds) with the individual laboratory standard deviation (rectangle). The color of the data point represents the analytical method employed. The solid blue line represents the consensus mean, and the green shaded region represents the $95 \%$ confidence interval for the consensus mean. The red solid lines represent the consensus range of tolerance, calculated as the values above and below the consensus mean that result in an acceptable $Z_{\text {comm }}^{\prime}$ score, $\left|Z_{\text {comm }}^{\prime}\right| \leq 2$. The red shaded region represents the NIST range of tolerance, which encompasses the target value bounded by its uncertainty ( $\left.U_{\text {NIST }}\right)$ and represents the range that results in an acceptable $Z_{\text {NIST }}$ score, $\left|Z_{\text {NIST }}\right| \leq 2$. 


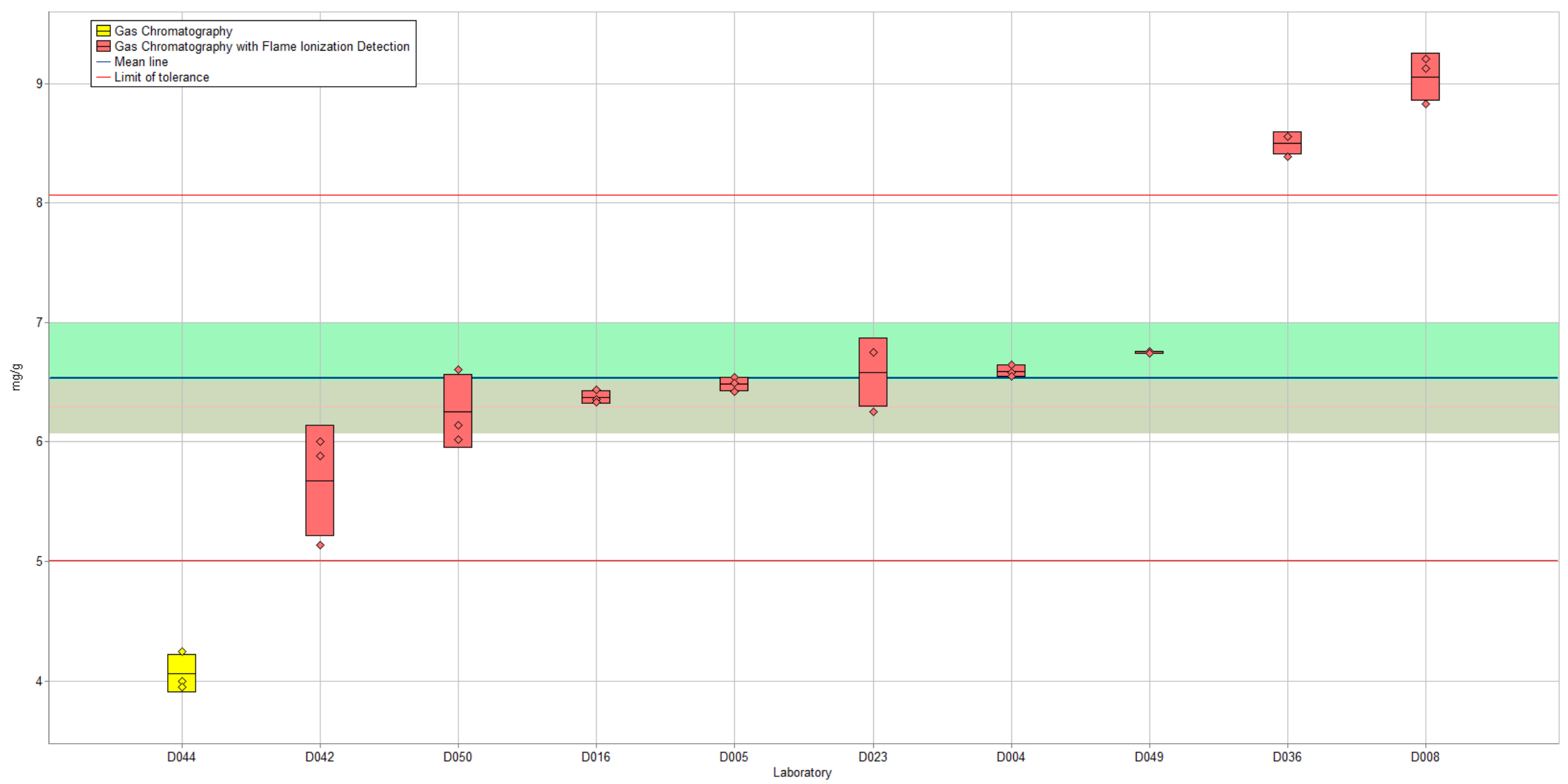

Figure 5-2. Total $\alpha$-linolenic acid in SRM 3275 Omega-3 and Omega-6 Fatty Acids in Fish Oil (Level 3) (data summary view analytical method). In this view, individual laboratory data are plotted (diamonds) with the individual laboratory standard deviation (rectangle). The color of the data point represents the analytical method employed. The solid blue line represents the consensus mean, and the green shaded region represents the $95 \%$ confidence interval for the consensus mean. The red solid lines represent the consensus range of tolerance, calculated as the values above and below the consensus mean that result in an acceptable $Z_{\text {comm }}^{\prime}$ score, $\left|Z_{\text {comm }}^{\prime}\right| \leq 2$. The red shaded region represents the NIST range of tolerance, which encompasses the target value bounded by its uncertainty ( $U_{\mathrm{NIST}}$ ) and represents the range that results in an acceptable $Z_{\text {NIST }}$ score, $\left|Z_{\text {NIST }}\right| \leq 2$. 
Exercise: HAMQAP Exercise 4 - Dietary Intake, Measurand: Total alpha-Linolenic Acid (C18:3 n-3)

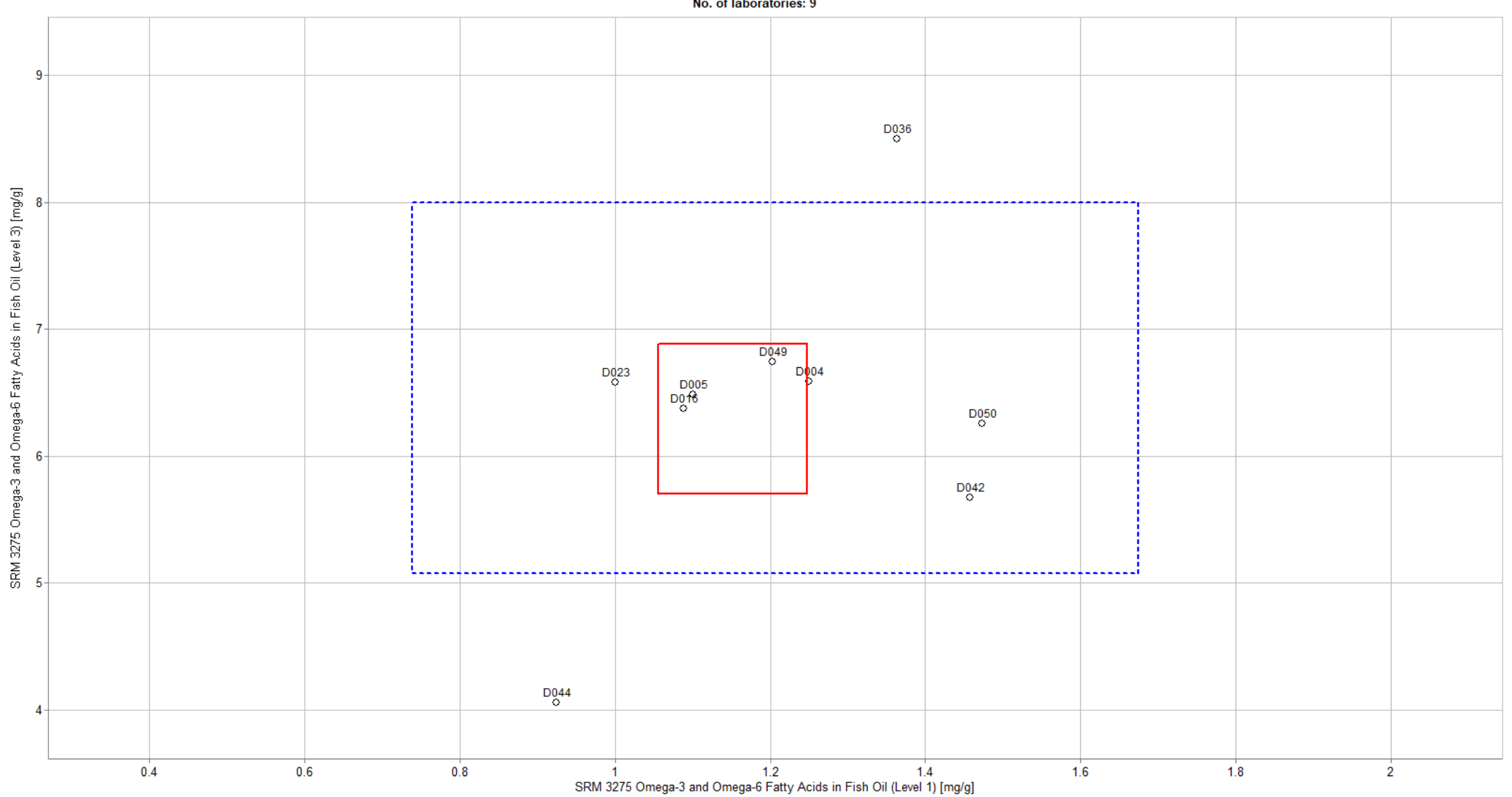

Figure 5-3. Laboratory means for total $\alpha$-linolenic acid in SRM 3275 Omega-3 and Omega-6 Fatty Acids in Fish Oil Level 1 and Level 3 (sample/sample comparison view). In this view, the individual laboratory mean for one sample (SRM 3275 Level 1) is compared to the individual laboratory mean for a second sample (SRM 3275 Level 3). The solid red box represents the NIST range of tolerance for the two samples, SRM 3275 Level 1 (x-axis) and SRM 3275 Level 3 (y-axis), which encompasses the target values bounded by their uncertainties ( $\left.U_{\mathrm{NIST}}\right)$ and represents the range that results in an acceptable $Z_{\mathrm{NIST}}$ score, $\left|Z_{\mathrm{NIST}}\right| \leq 2$. The dotted blue box represents the consensus range of tolerance for SRM 3275 Level 1 (x-axis) and SRM 3275 Level 3 (y-axis), calculated as the values above and below the consensus means that result in an acceptable $Z_{\text {comm }}^{\prime}$ score, $\left|Z_{\text {comm }}^{\prime}\right| \leq 2$. 
Table 5-3. Data summary table for total linoleic acid in fish oil. Data points highlighted in red have been flagged as potential outliers (e.g., Grubb and/or Cochran) by the NIST software package.

\begin{tabular}{|c|c|c|c|c|c|c|c|c|c|c|c|}
\hline & & \multicolumn{10}{|c|}{ Total Linoleic Acid (C18:2 n-6) } \\
\hline & & \multicolumn{5}{|c|}{$\begin{array}{l}\text { SRM } 3275 \text { Omega-3 and Omega-6 Fatty Acids in } \\
\text { Fish Oil (Level 1) (mg/g) }\end{array}$} & \multicolumn{5}{|c|}{$\begin{array}{l}\text { SRM } 3275 \text { Omega-3 and Omega-6 Fatty Acids in } \\
\text { Fish Oil (Level 3) (mg/g) }\end{array}$} \\
\hline & Lab & $\mathbf{A}$ & B & $\mathbf{C}$ & Avg & SD & $\mathbf{A}$ & $\mathbf{B}$ & $\mathbf{C}$ & Avg & SD \\
\hline \multirow{23}{*}{ 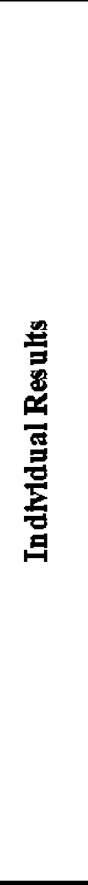 } & Target & & & & 2.20 & 0.18 & & & & 12.85 & 0.43 \\
\hline & D001 & & & & & & & & & & \\
\hline & D003 & & & & & & & & & & \\
\hline & D004 & 2.08 & 2.09 & 1.96 & 2.04 & 0.07 & 11.18 & 11.13 & 11.12 & 11.14 & 0.03 \\
\hline & D005 & 2.02 & 2.12 & 2.05 & 2.06 & 0.05 & 11.66 & 11.56 & 11.46 & 11.56 & 0.10 \\
\hline & D006 & & & & & & & & & & \\
\hline & D007 & & & & & & & & & & \\
\hline & D008 & 2.11 & 2.2 & 1.97 & 2.09 & 0.12 & 11.5 & 11.8 & 12.1 & 11.80 & 0.30 \\
\hline & D010 & & & & & & & & & & \\
\hline & D016 & 3.2953 & 3.19054 & 2.95244 & 3.15 & 0.18 & 18.17179 & 17.79083 & 17.68607 & 17.88 & 0.26 \\
\hline & D018 & & & & & & & & & & \\
\hline & D023 & 2 & 2 & 2 & 2.00 & 0.00 & 12 & 12.25 & 11.75 & 12.00 & 0.25 \\
\hline & D029 & & & & & & & & & & \\
\hline & D034 & & & & & & & & & & \\
\hline & D036 & & & & & & & & & & \\
\hline & D037 & & & & & & & & & & \\
\hline & D039 & & & & & & & & & & \\
\hline & D040 & & & & & & & & & & \\
\hline & D042 & 1.07 & 1.36 & 0.99 & 1.14 & 0.19 & 8.55 & 8.46 & 7.18 & 8.06 & 0.77 \\
\hline & D044 & 2.67 & 2.428 & 2.478 & 2.53 & 0.13 & 3.395 & 3.801 & 3.385 & 3.53 & 0.24 \\
\hline & D049 & 2.45 & 2.44 & 2.4 & 2.43 & 0.03 & 12 & 12.1 & 11.9 & 12.00 & 0.10 \\
\hline & D050 & 2.51 & 2.13 & 2.09 & 2.24 & 0.23 & 11.61 & 10.82 & 11 & 11.14 & 0.41 \\
\hline & D055 & & & & & & & & & & \\
\hline \multirow{5}{*}{ 害 } & & \multirow{5}{*}{\multicolumn{3}{|c|}{$\begin{array}{l}\text { Consensus Mean } \\
\text { Consensus Standard Deviation } \\
\text { Maximum } \\
\text { Minimum } \\
\text { N }\end{array}$}} & 2.20 & & \multirow{5}{*}{\multicolumn{3}{|c|}{$\begin{array}{l}\text { Consensus } \\
\text { Consensus } \\
\text { Maximum } \\
\text { Minimum } \\
\text { N }\end{array}$}} & 11.30 & \\
\hline & & & & & 0.11 & & & & & 0.41 & \\
\hline & & & & & 3.15 & & & & & 17.88 & \\
\hline & & & & & 1.14 & & & & & 3.53 & \\
\hline & & & & & 9 & & & & & 9 & \\
\hline
\end{tabular}


Exercise HAMQAP Exercise 4 - Dietary Intake
Sample:
SRM 3275 Omega-3 3 and Omega-6 Fatty Acids in Fish Oil (Level 1 )

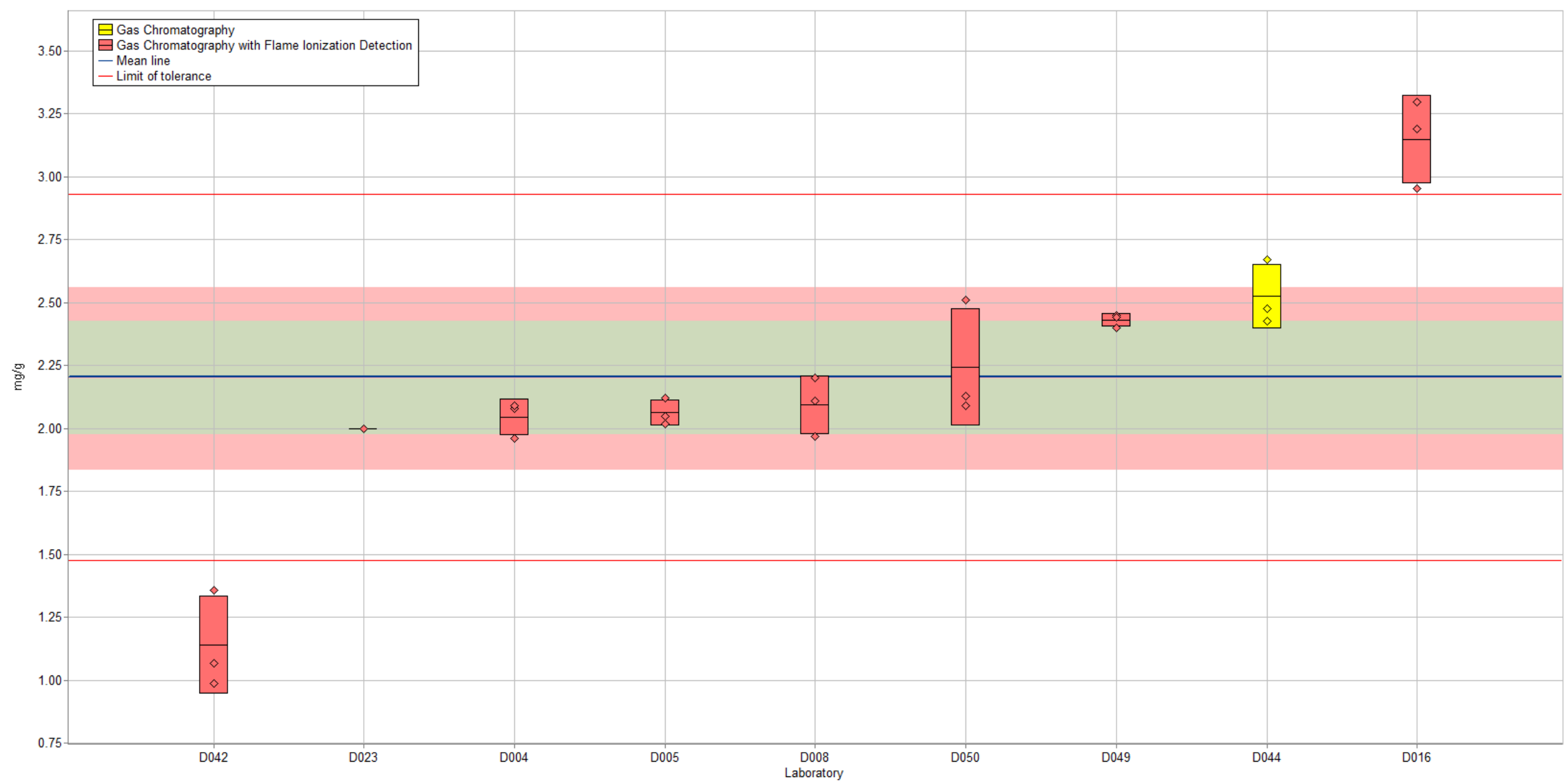

Figure 5-4. Total linoleic acid in SRM 3275 Omega-3 and Omega-6 Fatty Acids in Fish Oil (Level 1) (data summary view - analytical method). In this view, individual laboratory data are plotted (diamonds) with the individual laboratory standard deviation (rectangle). The color of the data point represents the analytical method employed. The solid blue line represents the consensus mean, and the green shaded region represents the $95 \%$ confidence interval for the consensus mean. The red solid lines represent the consensus range of tolerance, calculated as the values above and below the consensus mean that result in an acceptable $Z_{\text {comm }}^{\prime}$ score, $\left|Z_{\text {comm }}^{\prime}\right| \leq 2$. The red shaded region represents the NIST range of tolerance, which encompasses the target value bounded by its uncertainty ( $\left.U_{\text {NIST }}\right)$ and represents the range that results in an acceptable $Z_{\text {NIST }}$ score, $\left|Z_{\text {NIST }}\right| \leq 2$. 

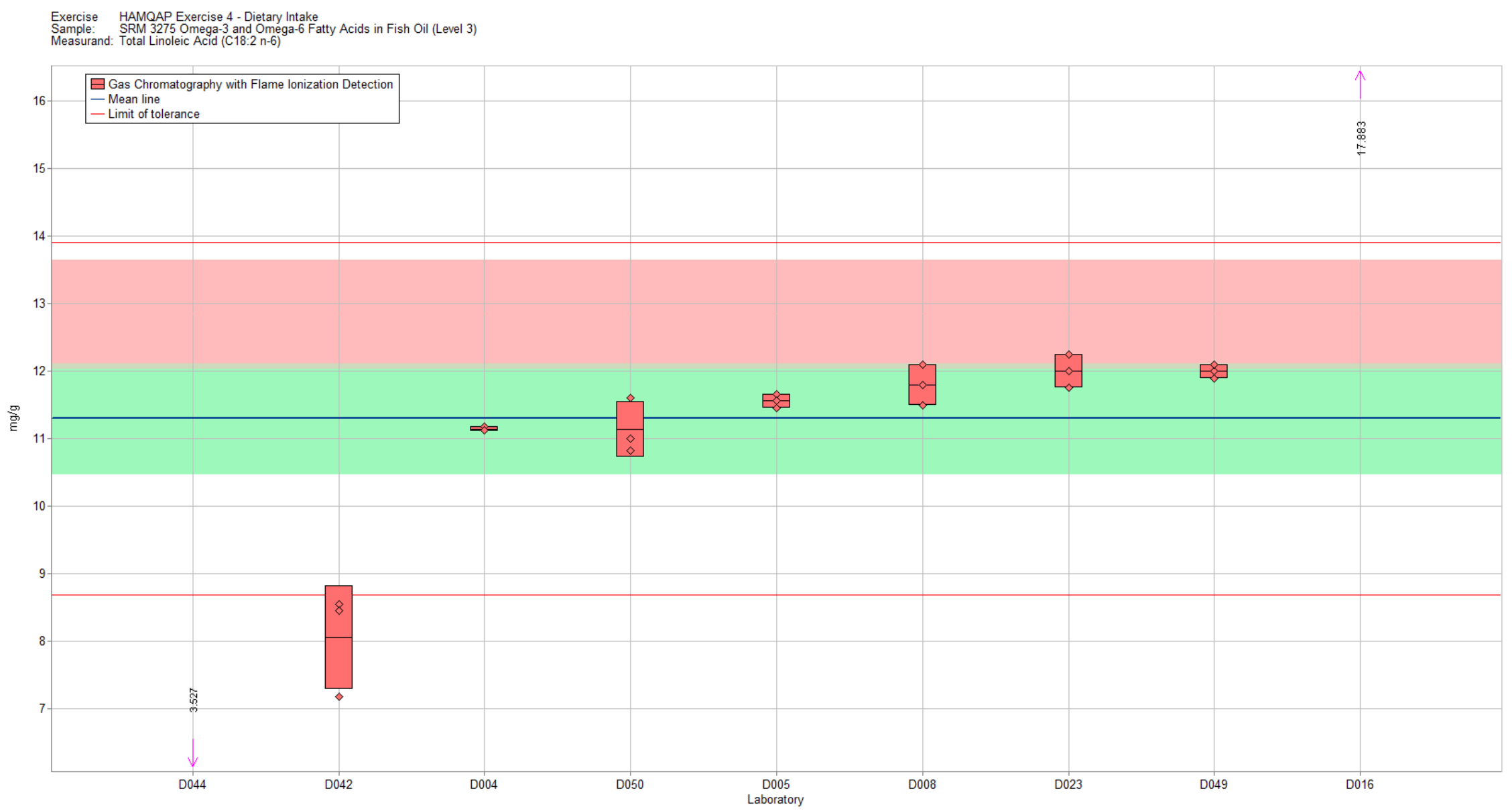

Figure 5-5. Total linoleic acid in SRM 3275 Omega-3 and Omega-6 Fatty Acids in Fish Oil (Level 3) (data summary view - analytical method). In this view, individual laboratory data are plotted (diamonds) with the individual laboratory standard deviation (rectangle). The color of the data point represents the analytical method employed. The solid blue line represents the consensus mean, and the green shaded region represents the $95 \%$ confidence interval for the consensus mean. The red solid lines represent the consensus range of tolerance, calculated as the values above and below the consensus mean that result in an acceptable $Z_{\text {comm }}^{\prime}$ score, $\left|Z_{\text {comm }}^{\prime}\right| \leq 2$. The red shaded region represents the NIST range of tolerance, which encompasses the target value bounded by its uncertainty ( $\left.U_{\text {NIST }}\right)$ and represents the range that results in an acceptable $Z_{\mathrm{NIST}}$ score, $\left|Z_{\mathrm{NIST}}\right| \leq 2$. 


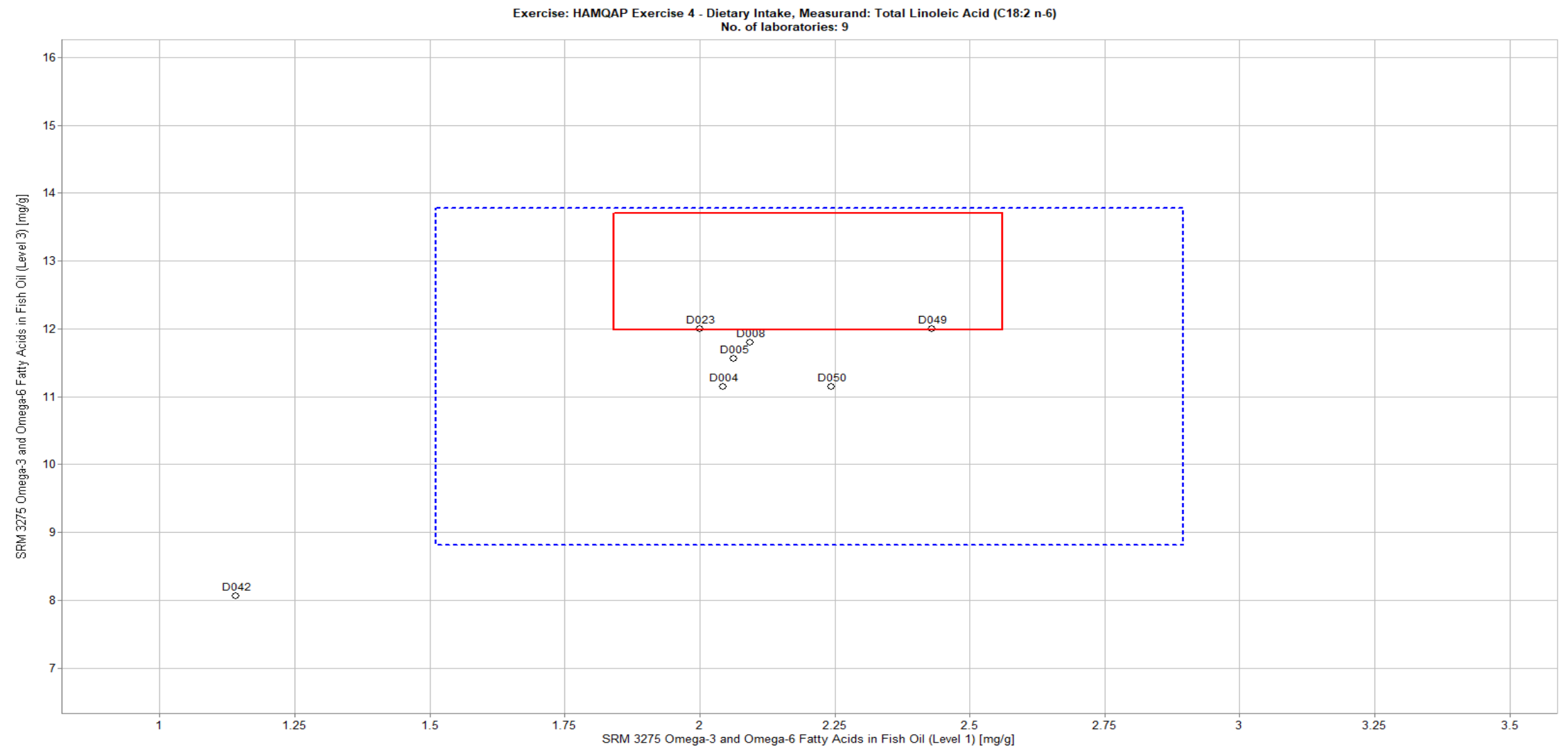

Figure 5-6. Laboratory means for total linoleic acid in SRM 3275 Omega-3 and Omega-6 Fatty Acids in Fish Oil Level 1 and Level 3 (sample/sample comparison view). In this view, the individual laboratory mean for one sample (SRM 3275 Level 1) is compared to the individual laboratory mean for a second sample (SRM 3275 Level 3). The solid red box represents the NIST range of tolerance for the two samples, SRM 3275 Level 1 (x-axis) and SRM 3275 Level 3 (y-axis), which encompasses the target values bounded by their uncertainties ( $U_{\mathrm{NIST}}$ ) and represents the range that results in an acceptable $Z_{\text {NIST }}$ score, $\left|Z_{\text {NIST }}\right| \leq 2$. The dotted blue box represents the consensus range of tolerance for SRM 3275 Level 1 (x-axis) and SRM 3275 Level 3 (y-axis), calculated as the values above and below the consensus means that result in an acceptable $Z_{\text {comm }}^{\prime}$ score, $\left|Z_{\text {comm }}^{\prime}\right| \leq 2$. 
Table 5-4. Data summary table for total arachidic acid in fish oil.

\begin{tabular}{|c|c|c|c|c|c|c|c|c|c|c|c|}
\hline & \multirow[b]{3}{*}{ Lab } & \multicolumn{10}{|c|}{ Total Arachidic Acid (C20:0) } \\
\hline & & \multicolumn{5}{|c|}{$\begin{array}{c}\text { SRM } 3275 \text { Omega-3 and Omega-6 Fatty Acids } \\
\text { in Fish Oil (Level 1) (mg/g) }\end{array}$} & \multicolumn{5}{|c|}{$\begin{array}{c}\text { SRM } 3275 \text { Omega-3 and Omega-6 Fatty Acids } \\
\text { in Fish Oil (Level 3) }(\mathrm{mg} / \mathrm{g})\end{array}$} \\
\hline & & $\mathbf{A}$ & $\bar{B}$ & $\mathbf{C}$ & $\overline{\text { Avg }}$ & SD & $\mathbf{A}$ & $\bar{B}$ & $\mathbf{C}$ & Avg & SD \\
\hline \multirow{22}{*}{ 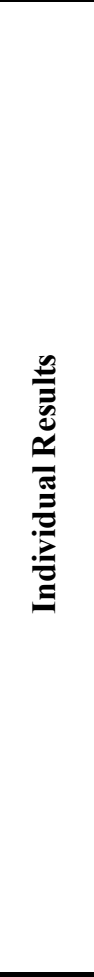 } & Target & & & & 1.828 & 0.068 & & & & 1.09 & 0.25 \\
\hline & D001 & & & & & & & & & & \\
\hline & D003 & & & & & & & & & & \\
\hline & D004 & 2.74 & 2.75 & 2.73 & 2.740 & 0.010 & 1.6 & 1.6 & 1.58 & 1.59 & 0.01 \\
\hline & D005 & 2.86 & 2.77 & 2.79 & 2.807 & 0.047 & 1.68 & 1.76 & 1.7 & 1.71 & 0.04 \\
\hline & D006 & & & & & & & & & & \\
\hline & D007 & & & & & & & & & & \\
\hline & D008 & $<4.00$ & $<4.00$ & $<4.00$ & & & $<4.00$ & $<4.00$ & $<4.00$ & & \\
\hline & D010 & & & & & & & & & & \\
\hline & D016 & 3.30165 & 3.26337 & 3.27294 & 3.279 & 0.020 & 1.99056 & 1.98099 & 2.02884 & 2.00 & 0.03 \\
\hline & D018 & & & & & & & & & & \\
\hline & D023 & 3 & 3 & 3 & 3.000 & 0.000 & 1.75 & 1.75 & 1.75 & 1.75 & 0.00 \\
\hline & D029 & & & & & & & & & & \\
\hline & D034 & & & & & & & & & & \\
\hline & D036 & 4.27 & 4.29 & 4.29 & 4.283 & 0.012 & 2.39 & 2.42 & 2.43 & 2.41 & 0.02 \\
\hline & D037 & & & & & & & & & & \\
\hline & D039 & & & & & & & & & & \\
\hline & D040 & & & & & & & & & & \\
\hline & D042 & 1.35 & 1.69 & 1.23 & 1.423 & 0.239 & 1.08 & 1.09 & 0.92 & 1.03 & 0.10 \\
\hline & D049 & 2.78 & 2.83 & 2.81 & 2.807 & 0.025 & 1.72 & 1.72 & 1.7 & 1.71 & 0.01 \\
\hline & D050 & 3.94 & 2.98 & 3.05 & 3.323 & 0.535 & 2.68 & 2.24 & 2.14 & 2.35 & 0.29 \\
\hline & D055 & & & & & & & & & & \\
\hline \multirow{5}{*}{ 氡 } & & \multirow{5}{*}{\multicolumn{3}{|c|}{$\begin{array}{l}\text { Consensus Mean } \\
\text { Consensus Standard Deviation } \\
\text { Maximum } \\
\text { Minimum } \\
\mathrm{N}\end{array}$}} & 2.993 & & \multirow{5}{*}{\multicolumn{3}{|c|}{$\begin{array}{l}\text { Consensus Mean } \\
\text { Consensus Standard Deviation } \\
\text { Maximum } \\
\text { Minimum } \\
\mathrm{N}\end{array}$}} & 1.83 & \\
\hline & & & & & 0.188 & & & & & 0.18 & \\
\hline & & & & & 4.283 & & & & & 2.41 & \\
\hline & & & & & 1.423 & & & & & 1.03 & \\
\hline & & & & & 8 & & & & & 8 & \\
\hline
\end{tabular}




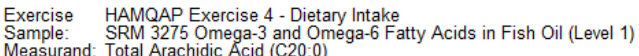

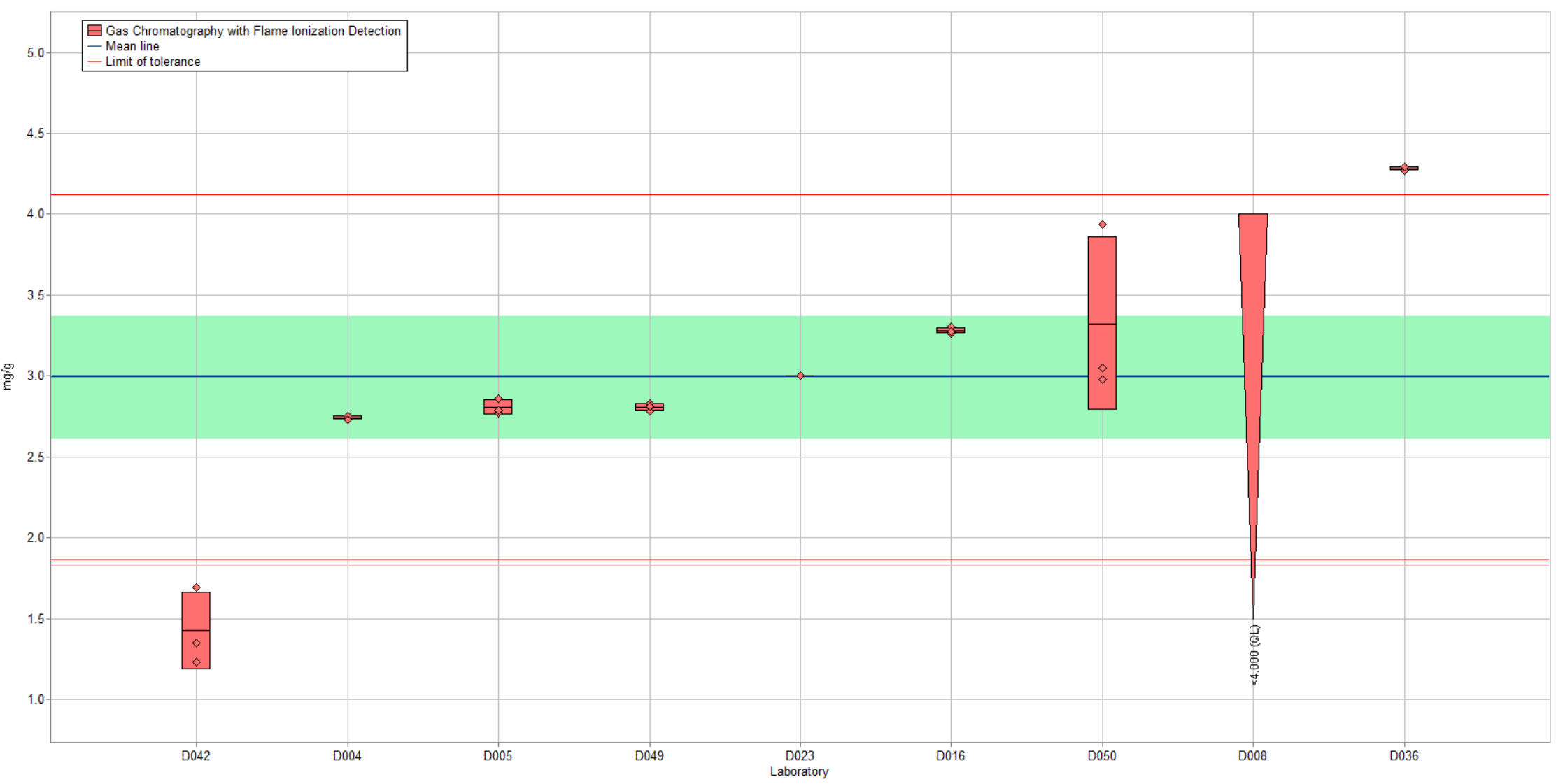

Figure 5-7. Total arachidic acid in SRM 3275 Omega-3 and Omega-6 Fatty Acids in Fish Oil (Level 1) (data summary view - analytical method). In this view, individual laboratory data are plotted (diamonds) with the individual laboratory standard deviation (rectangle). The color of the data point represents the analytical method employed. The solid blue line represents the consensus mean, and the green shaded region represents the $95 \%$ confidence interval for the consensus mean. The red solid lines represent the consensus range of tolerance, calculated as the values above and below the consensus mean that result in an acceptable $Z_{\text {comm }}^{\prime}$ score, $\left|Z_{\text {comm }}^{\prime}\right| \leq 2$. The red shaded region (thin red line below the lower limit of tolerance) represents the NIST range of tolerance, which encompasses the target value bounded by its uncertainty $\left(U_{\mathrm{NIST}}\right)$ and represents the range that results in an acceptable $Z_{\mathrm{NIST}}$ score, $\left|Z_{\mathrm{NIST}}\right| \leq 2$. 
Exercise HAMQAP Exercise 4 - Dietary Intake
Sample:
SRM 3275 Omega-3 and Omega-6 Fatty Acids in Fish Oil (Level 3)

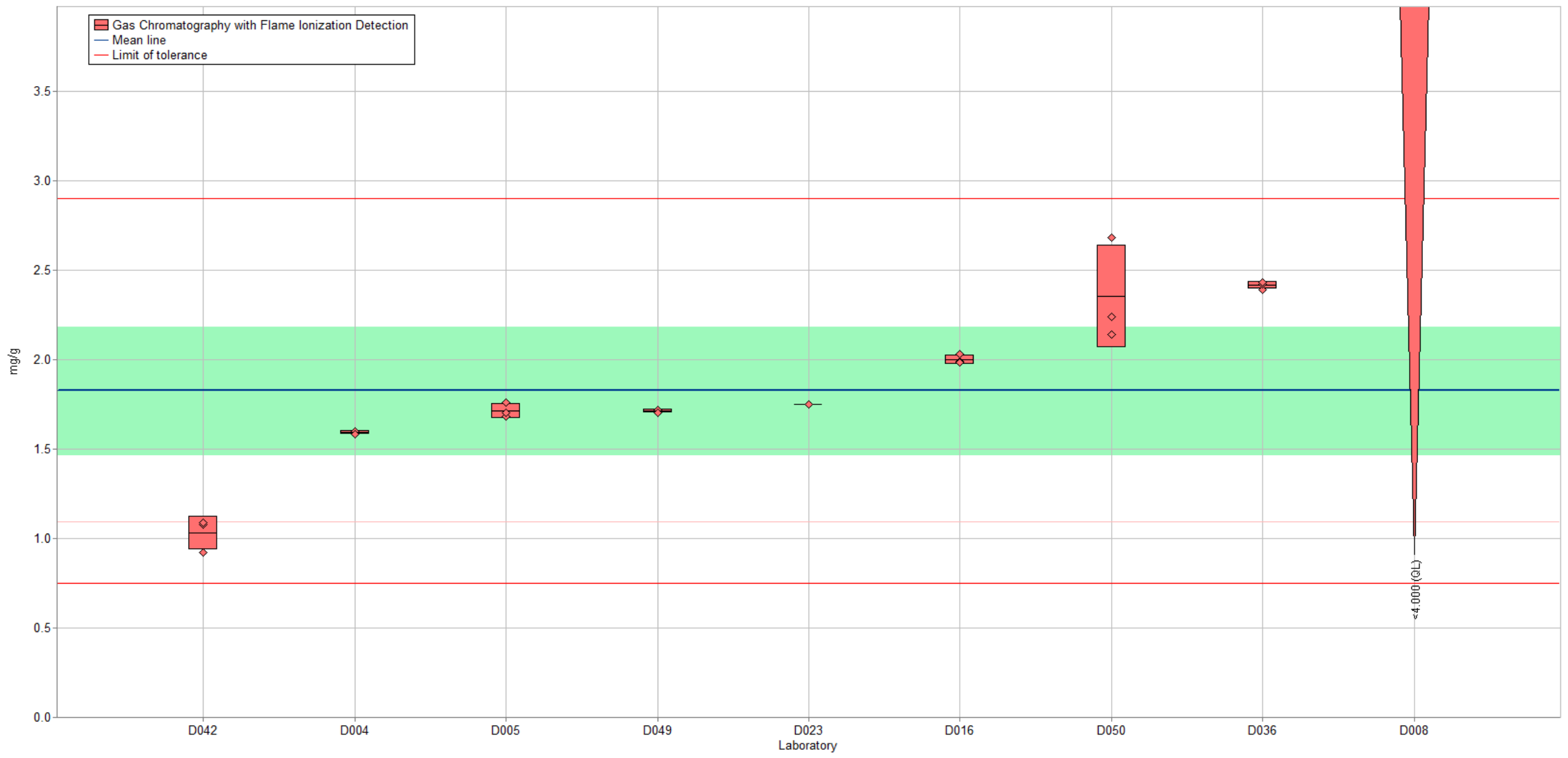

Figure 5-8. Total arachidic acid in SRM 3275 Omega-3 and Omega-6 Fatty Acids in Fish Oil (Level 3) (data summary view - analytical method). In this view, individual laboratory data are plotted (diamonds) with the individual laboratory standard deviation (rectangle). The color of the data point represents the analytical method employed. The solid blue line represents the consensus mean, and the green shaded region represents the $95 \%$ confidence interval for the consensus mean. The red solid lines represent the consensus range of tolerance, calculated as the values above and below the consensus mean that result in an acceptable $Z_{\text {comm }}^{\prime}$ score, $\left|Z_{\text {comm }}^{\prime}\right| \leq 2$. The red shaded region (thin red line above the lower limit of tolerance) represents the NIST range of tolerance, which encompasses the target value bounded by its uncertainty $\left(U_{\mathrm{NIST}}\right)$ and represents the range that results in an acceptable $Z_{\mathrm{NIST}}$ score, $\left|Z_{\mathrm{NIST}}\right| \leq 2$. 


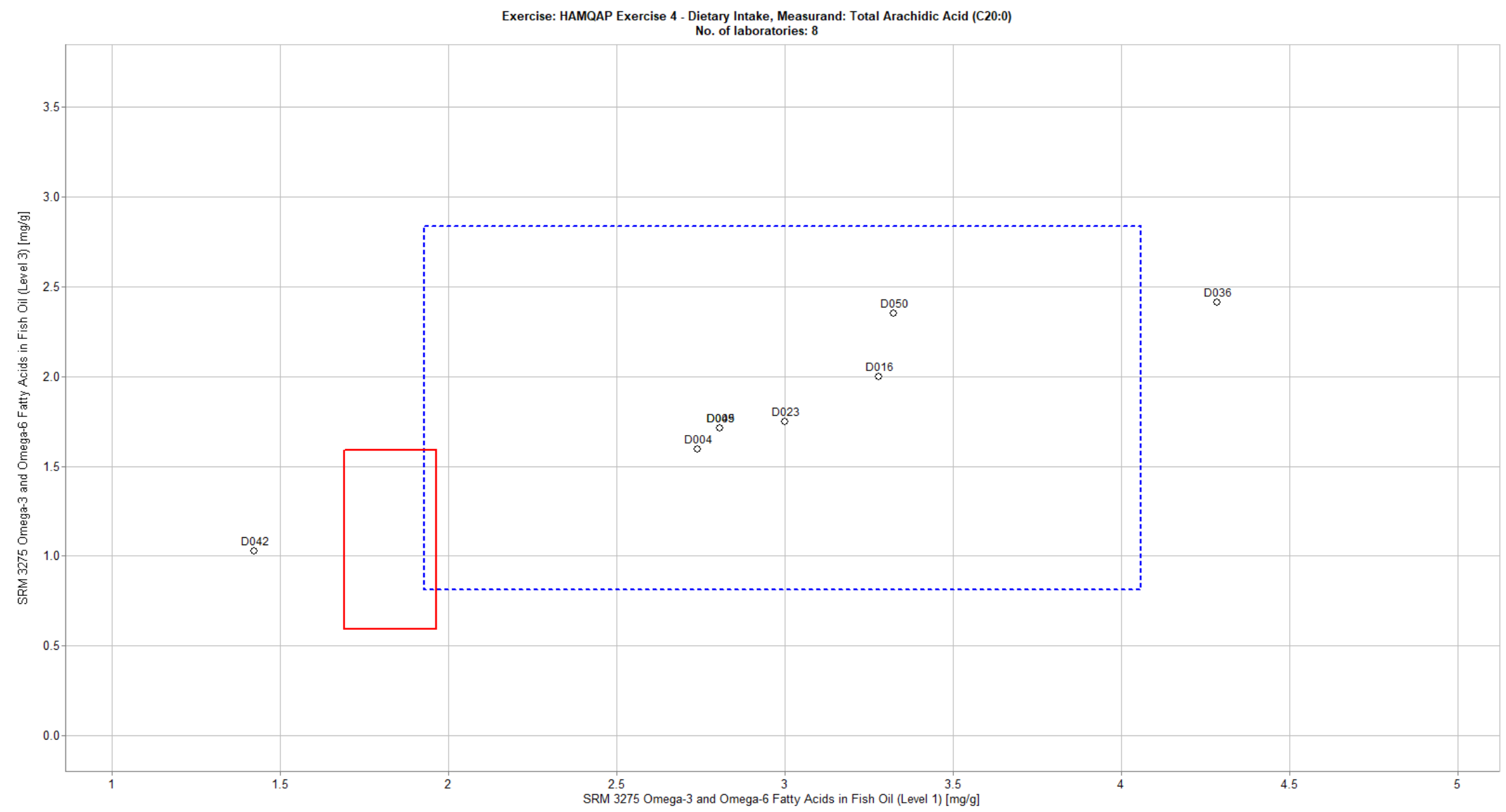

Figure 5-9. Laboratory means for total arachidic acid in SRM 3275 Omega-3 and Omega-6 Fatty Acids in Fish Oil Level 1 and Level 3 (sample/sample comparison view). In this view, the individual laboratory mean for one sample (SRM 3275 Level 1) is compared to the individual laboratory mean for a second sample (SRM 3275 Level 3). The solid red box represents the NIST range of tolerance for the two samples, SRM 3275 Level 1 (x-axis) and SRM 3275 Level 3 (y-axis), which encompasses the target values bounded by their uncertainties ( $U_{\text {NIST }}$ ) and represents the range that results in an acceptable $Z_{\text {NIST }}$ score, $\left|Z_{\text {NIST }}\right| \leq 2$. The dotted blue box represents the consensus range of tolerance for SRM 3275 Level 1 (x-axis) and SRM 3275 Level 3 (y-axis), calculated as the values above and below the consensus means that result in an acceptable $Z_{\text {comm }}^{\prime}$ score, $\left|Z_{\text {comm }}^{\prime}\right| \leq 2$. 
Table 5-5. Data summary table for total EPA in fish oil. Data points highlighted in red have been flagged as potential outliers (e.g., Grubb and/or Cochran) by the NIST software package.

\begin{tabular}{|c|c|c|c|c|c|c|c|c|c|c|c|}
\hline & & \multicolumn{10}{|c|}{ Total EPA (C20:5 n-3) } \\
\hline & & \multicolumn{5}{|c|}{$\begin{array}{l}\text { SRM } 3275 \text { Omega-3 and Omega-6 Fatty Acids in } \\
\text { Fish Oil (Level 1) }(\mathrm{mg} / \mathrm{g})\end{array}$} & \multicolumn{5}{|c|}{$\begin{array}{l}\text { SRM } 3275 \text { Omega-3 and Omega-6 Fatty Acids in } \\
\text { Fish Oil (Level 3) (mg/g) }\end{array}$} \\
\hline & Lab & $\mathbf{A}$ & B & $\mathbf{C}$ & Avg & SD & $\mathbf{A}$ & B & $\mathbf{C}$ & Avg & SD \\
\hline \multirow{23}{*}{ 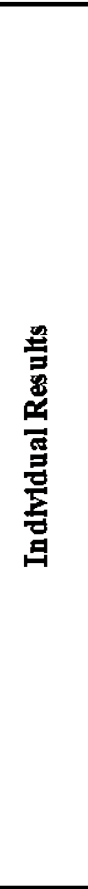 } & Target & & & & 108 & 11 & & & & 153.3 & 9.0 \\
\hline & D001 & & & & & & & & & & \\
\hline & D003 & & & & & & & & & & \\
\hline & D004 & 104.97 & 104.87 & 104.47 & 105 & 0 & 143.26 & 143.02 & 142.89 & 143.1 & 0.2 \\
\hline & D005 & 104 & 109 & 103 & 105 & 3 & 158 & 152 & 151 & 153.7 & 3.8 \\
\hline & D006 & 102 & 102 & 103 & 102 & 1 & 153 & 154 & 154 & 153.7 & 0.6 \\
\hline & D007 & & & & & & & & & & \\
\hline & D008 & 104 & 105 & 104 & 104 & 1 & 149 & 146 & 150 & 148.3 & 2.1 \\
\hline & D010 & & & & & & & & & & \\
\hline & D016 & 107.7248 & 107.3365 & 107.4559 & 108 & 0 & 155.9665 & 155.5283 & 155.9665 & 155.8 & 0.3 \\
\hline & D018 & & & & & & & & & & \\
\hline & D023 & 115.5 & 116.75 & 117.25 & 117 & 1 & 158.75 & 164.5 & 158 & 160.4 & 3.6 \\
\hline & D029 & & & & & & & & & & \\
\hline & D034 & & & & & & & & & & \\
\hline & D036 & & & & & & & & & & \\
\hline & D037 & & & & & & & & & & \\
\hline & D039 & & & & & & & & & & \\
\hline & D040 & & & & & & & & & & \\
\hline & D042 & 59.1 & 73.6 & 54.1 & 62 & 10 & 113 & 115 & 98.4 & 108.8 & 9.1 \\
\hline & D044 & 118.275 & 110.424 & 114.255 & 114 & 4 & 106.134 & 112.455 & 104.176 & 107.6 & 4.3 \\
\hline & D049 & 107 & 107 & 108 & 107 & 1 & 154 & 153 & 157 & 154.7 & 2.1 \\
\hline & D050 & 106.73 & 108.92 & 108.59 & 108 & 1 & 151.18 & 146.18 & 146.47 & 147.9 & 2.8 \\
\hline & D055 & & & & & & & & & & \\
\hline \multirow{5}{*}{ 害 } & & \multirow{5}{*}{\multicolumn{3}{|c|}{$\begin{array}{l}\text { Consensus Mean } \\
\text { Consensus Standard Deviation } \\
\text { Maximum } \\
\text { Minimum } \\
\text { N }\end{array}$}} & 108 & & \multirow{5}{*}{\multicolumn{3}{|c|}{$\begin{array}{l}\text { Consensus } \\
\text { Consensus } \\
\text { Maximum } \\
\text { Minimum } \\
\text { N }\end{array}$}} & 151.4 & \\
\hline & & & & & 2 & & & & & 3.2 & \\
\hline & & & & & 117 & & & & & 160.4 & \\
\hline & & & & & 62 & & & & & 107.6 & \\
\hline & & & & & 10 & & & & & 10 & \\
\hline
\end{tabular}


Exercise HAMQAP Exercise 4 - Dietary Intake
Sample:
MRM 3275 Omegag-3 and Omega-6 Fatty Acids in Fish Oil (Level 1)
Measurand: Total EPA (C20.5 $\mathrm{n}-3$ )

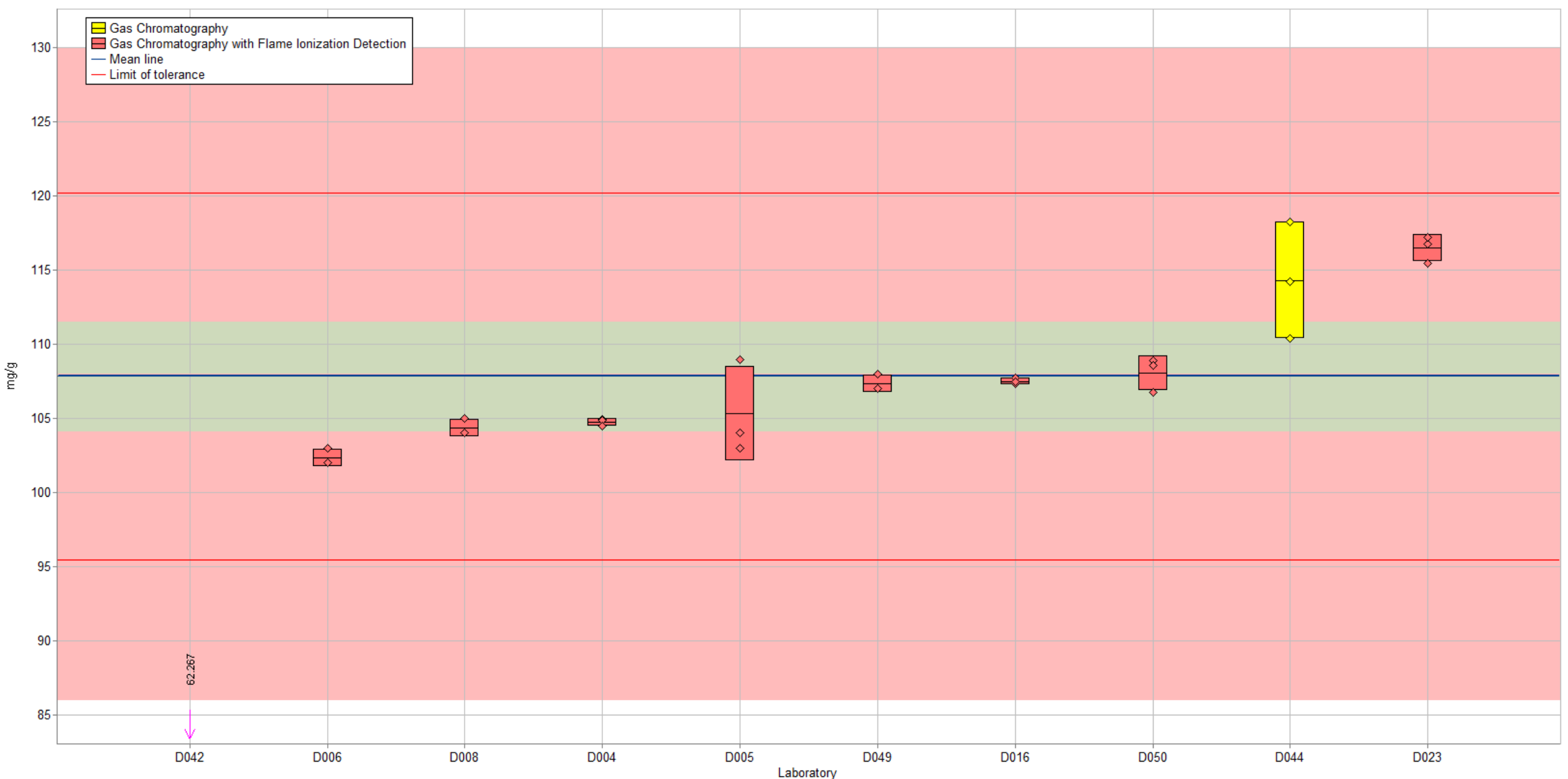

Figure 5-10. Total EPA in SRM 3275 Omega-3 and Omega-6 Fatty Acids in Fish Oil (Level 1) (data summary view - analytical method). In this view, individual laboratory data are plotted (diamonds) with the individual laboratory standard deviation (rectangle). The color of the data point represents the analytical method employed. The solid blue line represents the consensus mean, and the green shaded region represents the $95 \%$ confidence interval for the consensus mean. The red solid lines represent the consensus range of tolerance, calculated as the values above and below the consensus mean that result in an acceptable $Z_{\text {comm }}^{\prime}$ score, $\left|Z_{\text {comm }}^{\prime}\right| \leq 2$. The red shaded region represents the NIST range of tolerance, which encompasses the target value bounded by its uncertainty ( $\left.U_{\text {NIST }}\right)$ and represents the range that results in an acceptable $Z_{\mathrm{NIST}}$ score, $\left|Z_{\mathrm{NIST}}\right| \leq 2$. 
Exercise HAMQAP Exercise 4 - Dietary Intake
Sample:
MRM 3275 OMmega-3 and Omega-6 Fatty Acids in Fish Oil (Level 3$)$
Measurand: Total EPA (C20:5 $\mathrm{n}-3$ )

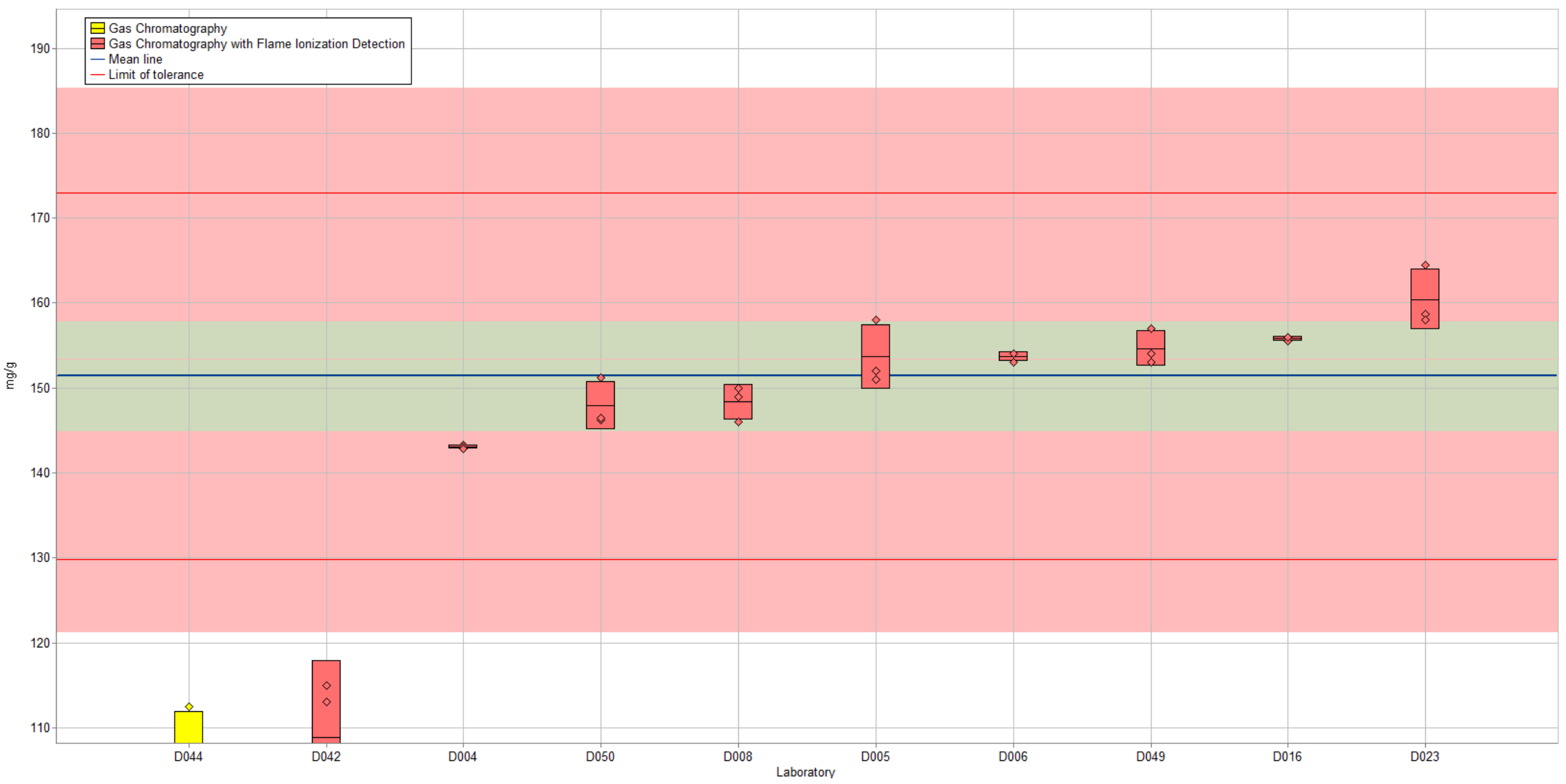

Figure 5-11. Total EPA in SRM 3275 Omega-3 and Omega-6 Fatty Acids in Fish Oil (Level 3) (data summary view - analytical method). In this view, individual laboratory data are plotted (diamonds) with the individual laboratory standard deviation (rectangle). The color of the data point represents the analytical method employed. The solid blue line represents the consensus mean, and the green shaded region represents the $95 \%$ confidence interval for the consensus mean. The red solid lines represent the consensus range of tolerance, calculated as the values above and below the consensus mean that result in an acceptable $Z_{\text {comm }}^{\prime}$ score, $\left|Z_{\text {comm }}^{\prime}\right| \leq 2$. The red shaded region represents the NIST range of tolerance, which encompasses the target value bounded by its uncertainty ( $\left.U_{\text {NIST }}\right)$ and represents the range that results in an acceptable $Z_{\text {NIST }}$ score, $\left|Z_{\text {NIST }}\right| \leq 2$. 


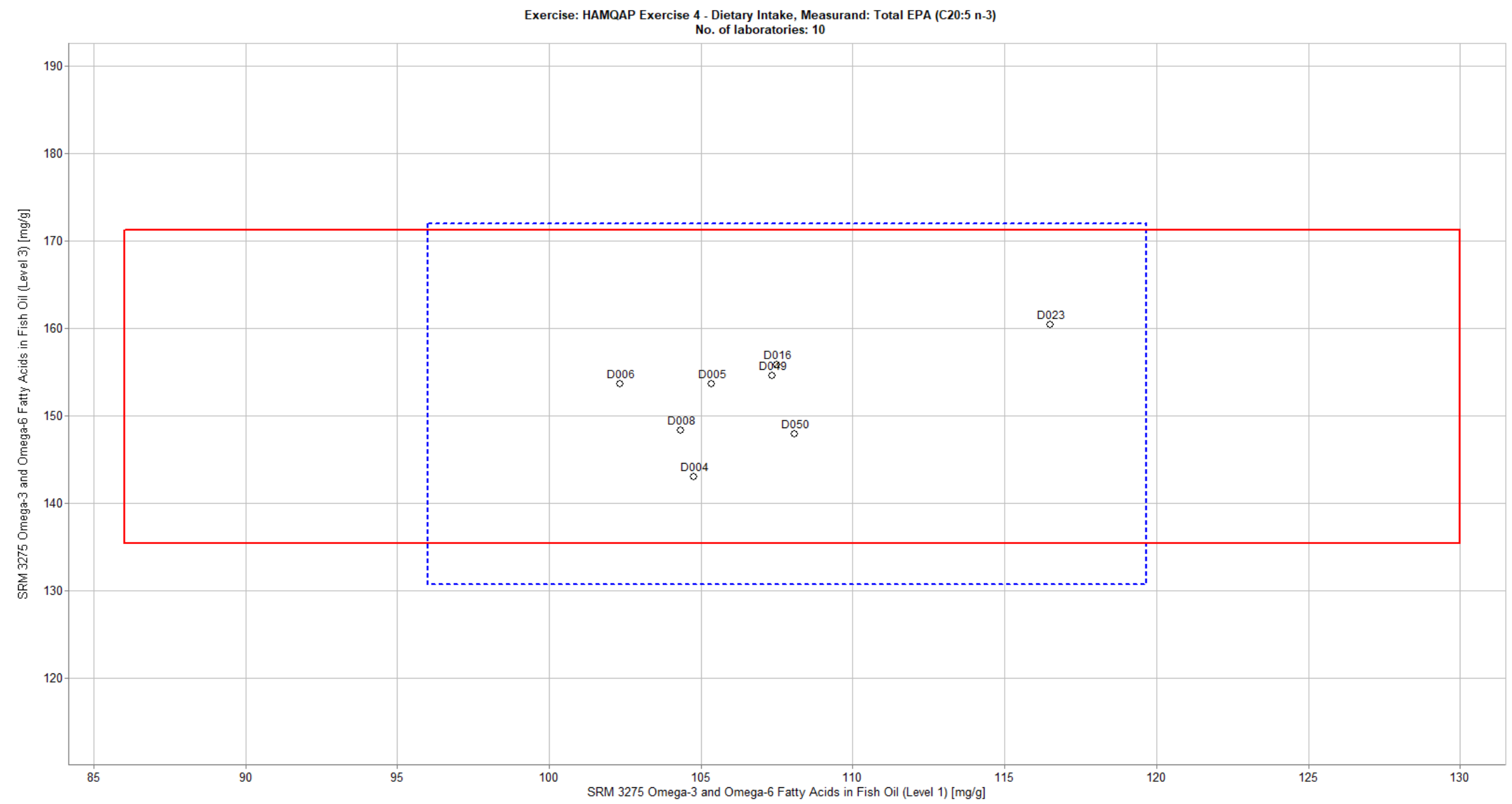

Figure 5-12. Laboratory means for total EPA in SRM 3275 Omega-3 and Omega-6 Fatty Acids in Fish Oil Level 1 and Level 3 (sample/sample comparison view). In this view, the individual laboratory mean for one sample (SRM 3275 Level 1) is compared to the individual laboratory mean for a second sample (SRM 3275 Level 3). The solid red box represents the NIST range of tolerance for the two samples, SRM 3275 Level 1 (x-axis) and SRM 3275 Level 3 (y-axis), which encompasses the target values bounded by their uncertainties ( $U_{\text {NIST }}$ ) and represents the range that results in an acceptable $Z_{\text {NIST }}$ score, $\left|Z_{\text {NIST }}\right| \leq 2$. The dotted blue box represents the consensus range of tolerance for SRM 3275 Level 1 (x-axis) and SRM 3275 Level 3 (y-axis), calculated as the values above and below the consensus means that result in an acceptable $Z_{\text {comm }}^{\prime}$ score, $\left|Z_{\text {comm }}^{\prime}\right| \leq 2$. 
Table 5-6. Data summary table for total DHA in fish oil. Data points highlighted in red have been flagged as potential outliers (e.g., Grubb and/or Cochran) by the NIST software package.

\begin{tabular}{|c|c|c|c|c|c|c|c|c|c|c|c|}
\hline & \multirow[b]{3}{*}{ Lab } & \multicolumn{10}{|c|}{ Total DHA (C22:6 n-3) } \\
\hline & & \multicolumn{5}{|c|}{$\begin{array}{l}\text { SRM } 3275 \text { Omega-3 and Omega-6 Fatty Acids in } \\
\text { Fish Oil (Level 1) (mg/g) }\end{array}$} & \multicolumn{5}{|c|}{$\begin{array}{c}\text { SRM } 3275 \text { Omega-3 and Omega-6 Fatty Acids in } \\
\text { Fish Oil (Level 3) (mg/g) }\end{array}$} \\
\hline & & $\overline{\mathbf{A}}$ & $\mathbf{B}$ & $\mathbf{C}$ & $\mathbf{A v g}$ & SD & $\overline{\mathbf{A}}$ & $\mathbf{B}$ & $\mathbf{C}$ & Avg & SD \\
\hline \multirow{23}{*}{ 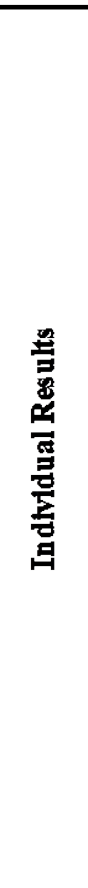 } & Target & & & & 411 & 14 & & & & 99.7 & 4.8 \\
\hline & D001 & & & & & & & & & & \\
\hline & D003 & & & & & & & & & & \\
\hline & D004 & 436.38 & 434.31 & 432.35 & 434 & 2 & 94.58 & 94.39 & 94.37 & 94.4 & 0.1 \\
\hline & D005 & 419 & 419 & 419 & 419 & 0 & 101 & 101 & 99.9 & 100.6 & 0.6 \\
\hline & D006 & 396 & 395 & 399 & 397 & 2 & 96 & 97 & 97 & 96.7 & 0.6 \\
\hline & D007 & & & & & & & & & & \\
\hline & D008 & 412 & 411 & 414 & 412 & 2 & 96.4 & 94 & 98.5 & 96.3 & 2.3 \\
\hline & D010 & & & & & & & & & & \\
\hline & D016 & 418.124 & 417.7308 & 418.8433 & 418 & 1 & 98.23037 & 98.04816 & 98.12488 & 98.1 & 0.1 \\
\hline & D018 & & & & & & & & & & \\
\hline & D023 & 443.5 & 456 & 447 & 449 & 6 & 98.5 & 105 & 101.25 & 101.6 & 3.3 \\
\hline & D029 & & & & & & & & & & \\
\hline & D034 & & & & & & & & & & \\
\hline & D036 & & & & & & & & & & \\
\hline & D037 & & & & & & & & & & \\
\hline & D039 & & & & & & & & & & \\
\hline & D040 & & & & & & & & & & \\
\hline & $\mathrm{D} 042$ & 200 & 244 & 184 & 209 & 31 & 61.5 & 64.2 & 54.4 & 60.0 & 5.1 \\
\hline & D044 & 500.745 & 470.49 & 486.465 & 486 & 15 & 55.479 & 59.91 & 54.465 & 56.6 & 2.9 \\
\hline & D049 & 420 & 416 & 418 & 418 & 2 & 99.2 & 101 & 101 & 100.4 & 1.0 \\
\hline & D050 & 411.28 & 421.52 & 419.54 & 417 & 5 & 95.28 & 91.88 & 92.4 & 93.2 & 1.8 \\
\hline & D055 & & & & & & & & & & \\
\hline \multirow{5}{*}{ 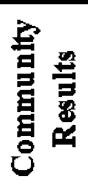 } & & \multirow{5}{*}{\multicolumn{3}{|c|}{$\begin{array}{l}\text { Consensus Mean } \\
\text { Consensus Standard Deviation } \\
\text { Maximum } \\
\text { Minimum } \\
\text { N }\end{array}$}} & 426 & & \multirow{5}{*}{\multicolumn{3}{|c|}{$\begin{array}{l}\text { Consensus } \\
\text { Consensus } \\
\text { Maximum } \\
\text { Minimum } \\
\text { N }\end{array}$}} & 97.7 & \\
\hline & & & & & 9 & & & & & 1.8 & \\
\hline & & & & & 486 & & & & & 101.6 & \\
\hline & & & & & 209 & & & & & 56.6 & \\
\hline & & & & & 10 & & & & & 10 & \\
\hline
\end{tabular}




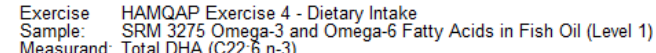

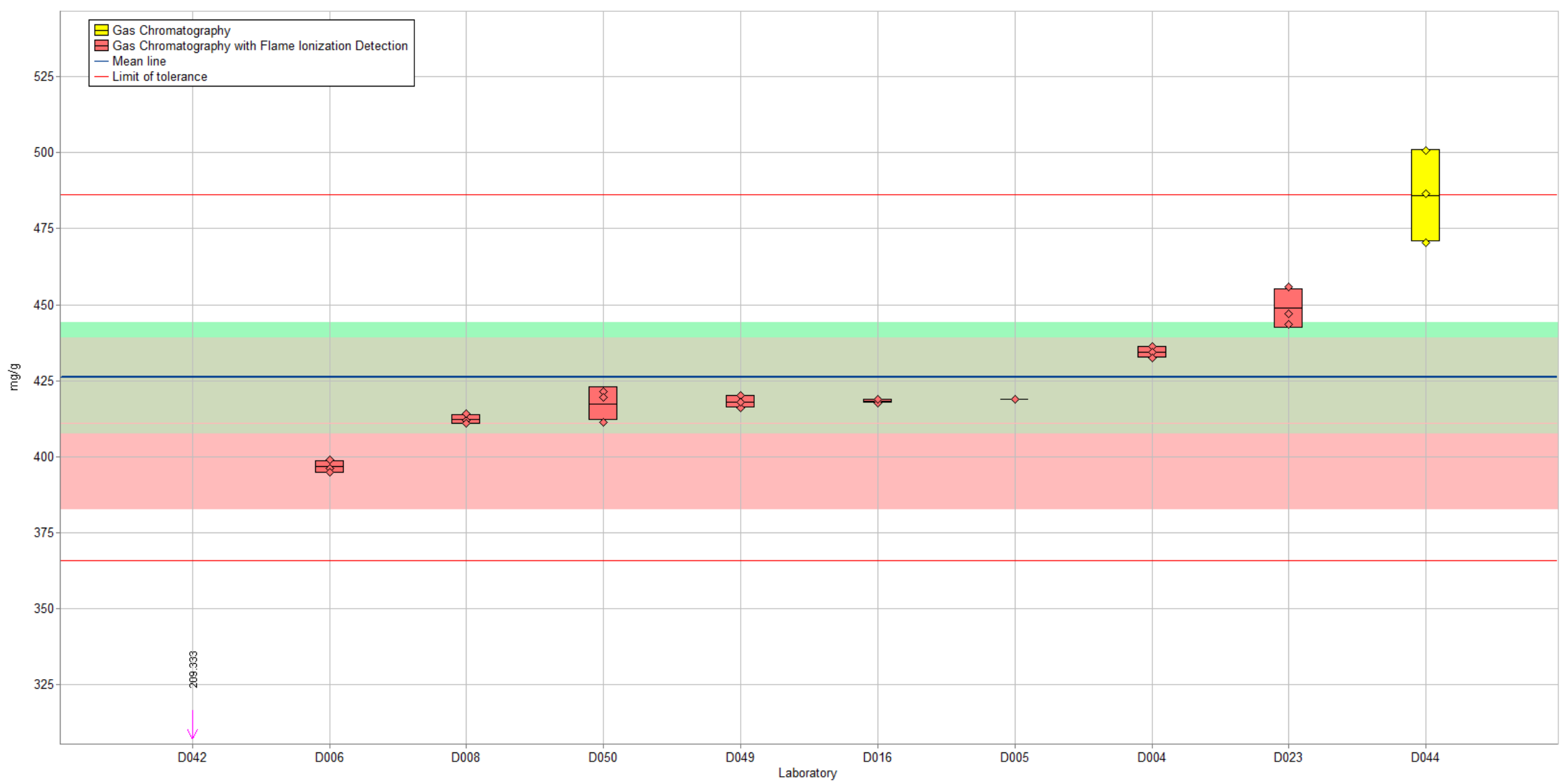

Figure 5-13. Total DHA in SRM 3275 Omega-3 and Omega-6 Fatty Acids in Fish Oil (Level 1) (data summary view - analytical method). In this view, individual laboratory data are plotted (diamonds) with the individual laboratory standard deviation (rectangle). The color of the data point represents the analytical method employed. The solid blue line represents the consensus mean, and the green shaded region represents the $95 \%$ confidence interval for the consensus mean. The red solid lines represent the consensus range of tolerance, calculated as the values above and below the consensus mean that result in an acceptable $Z_{\text {comm }}^{\prime}$ score, $\left|Z_{\text {comm }}^{\prime}\right| \leq 2$. The red shaded region represents the NIST range of tolerance, which encompasses the target value bounded by its uncertainty ( $\left.U_{\mathrm{NIST}}\right)$ and represents the range that results in an acceptable $Z_{\mathrm{NIST}}$ score, $\left|Z_{\mathrm{NIST}}\right| \leq 2$. 


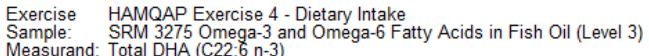

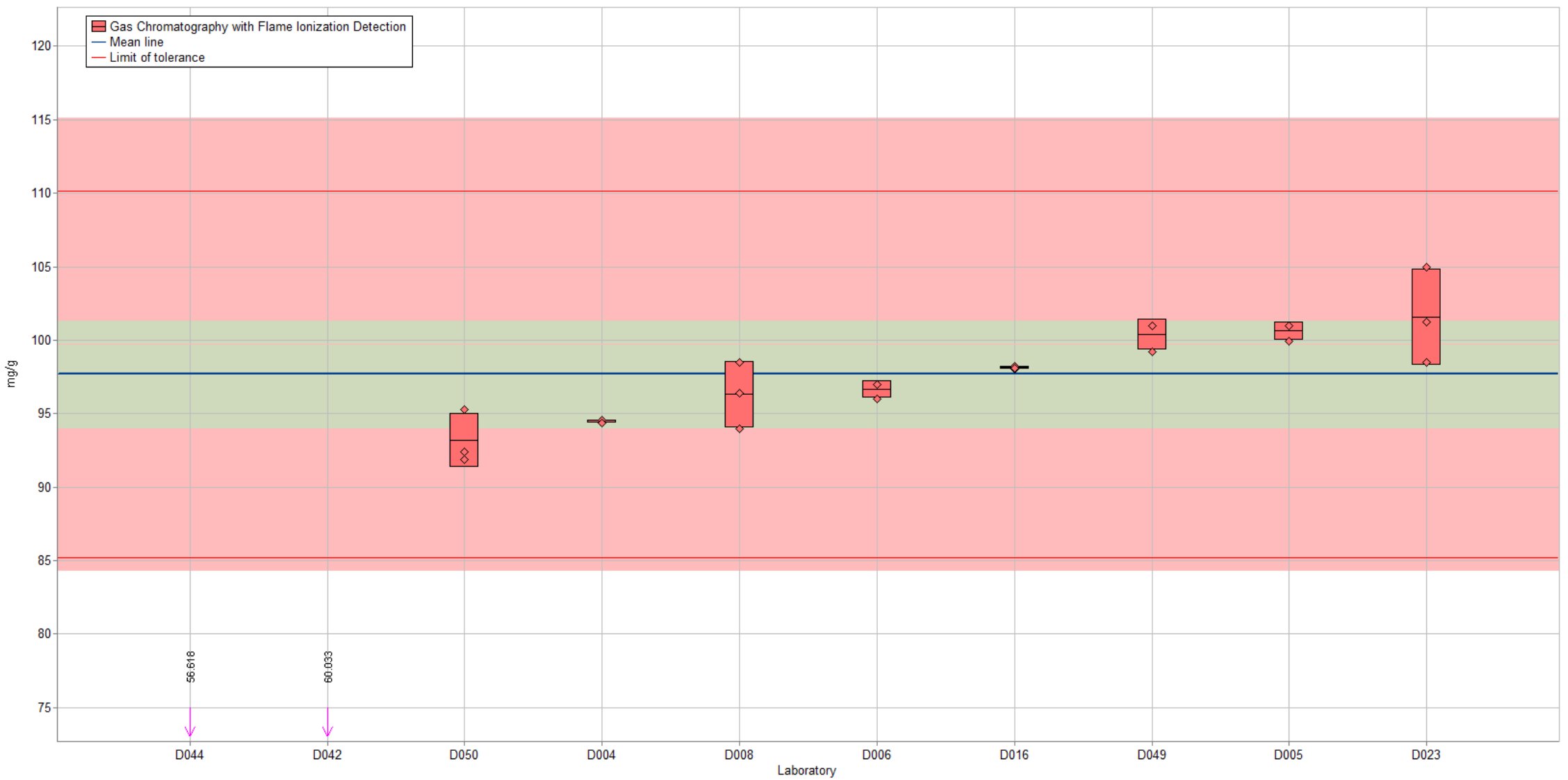

Figure 5-14. Total DHA in SRM 3275 Omega-3 and Omega-6 Fatty Acids in Fish Oil (Level 3) (data summary view - analytical method). In this view, individual laboratory data are plotted (diamonds) with the individual laboratory standard deviation (rectangle). The color of the data point represents the analytical method employed. The solid blue line represents the consensus mean, and the green shaded region represents the $95 \%$ confidence interval for the consensus mean. The red solid lines represent the consensus range of tolerance, calculated as the values above and below the consensus mean that result in an acceptable $Z_{\text {comm }}^{\prime}$ score, $\left|Z_{\text {comm }}^{\prime}\right| \leq 2$. The red shaded region represents the NIST range of tolerance, which encompasses the target value bounded by its uncertainty $\left(U_{\text {NIST }}\right)$ and represents the range that results in an acceptable $Z_{\text {NIST }}$ score, $\left|Z_{\text {NIST }}\right| \leq 2$. 
Exercise: HAMQAP Exercise 4 - Dietary Intake, Measurand: Total DHA (C22:6 n-3)

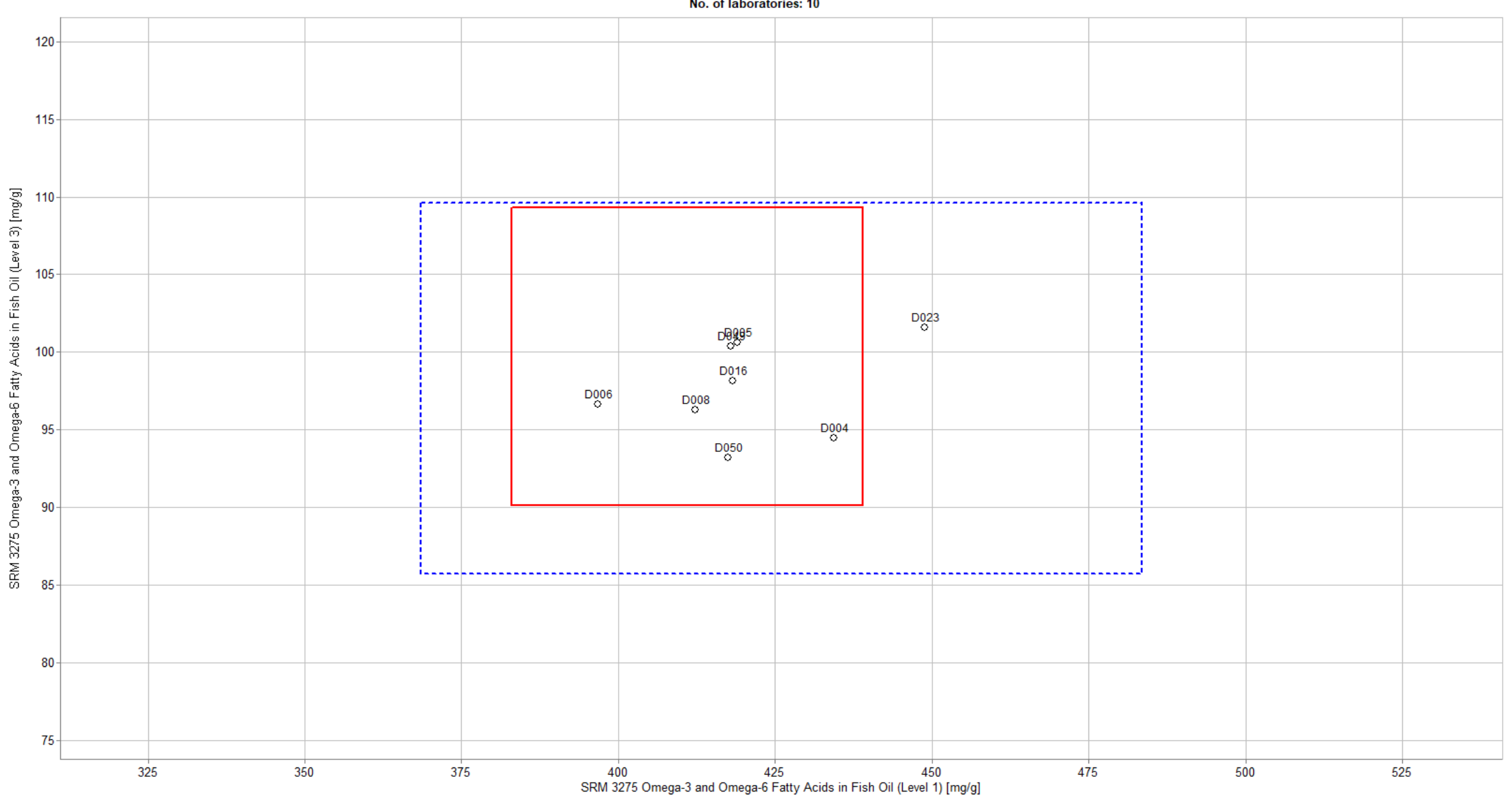

Figure 5-15. Laboratory means for total DHA in SRM 3275 Omega-3 and Omega-6 Fatty Acids in Fish Oil Level 1 and Level 3 (sample/sample comparison view). In this view, the individual laboratory mean for one sample (SRM 3275 Level 1) is compared to the individual laboratory mean for a second sample (SRM 3275 Level 3). The solid red box represents the NIST range of tolerance for the two samples, SRM 3275 Level 1 (x-axis) and SRM 3275 Level 3 (y-axis), which encompasses the target values bounded by their uncertainties ( $\left.U_{\mathrm{NIST}}\right)$ and represents the range that results in an acceptable $Z_{\mathrm{NIST}}$ score, $\left|Z_{\mathrm{NIST}}\right| \leq 2$. The dotted blue box represents the consensus range of tolerance for SRM 3275 Level 1 (x-axis) and SRM 3275 Level 3 (y-axis), calculated as the values above and below the consensus means that result in an acceptable $Z_{\text {comm }}^{\prime}$ score, $\left|Z_{\text {comm }}^{\prime}\right| \leq 2$. 
Human Metabolites Sample Information

Human Serum A and B. Participants were provided with three vials of SRM 2378 Fatty Acids in Frozen Human Serum Level 1 and three vials of SRM 2378 Fatty Acids in Frozen Human Serum Level 2, each containing $1 \mathrm{~mL}$ of frozen human serum. Level 1 was collected from three healthy donors who took $1000 \mathrm{mg}$ /day of fish oil supplements for a minimum of one month prior to collection, and Level 2 was collected from three healthy donors who took $1000 \mathrm{mg} /$ day of flaxseed oil supplements for a minimum of one month prior to collection. Participants were asked to avoid exposing the material to direct sun or UV light, to store the material at or below $-70{ }^{\circ} \mathrm{C}$, and to prepare one sample and report one value from each vial provided. Before use, participants were instructed to allow the material to thaw at room temperature for at least 30 min prior to sampling, use the material immediately after thawing, gently mix the contents prior to removal of a test portion for analysis, and use a sample size of at least $0.1 \mathrm{~g}$ to $0.5 \mathrm{~g}$. The approximate analyte levels were not reported to participants prior to the study. Certified values for EPA and DHA in SRM 2378 were assigned using results from NIST by GC-FID and GC-MS and from CDC by ID-GC-MS. Certified values for $\alpha$-linolenic acid and linoleic acid in SRM 2378 were assigned using results from NIST by GC-FID and from CDC by ID-GC-MS. Reference values for arachidic acid in SRM 2378 were assigned using results from NIST by GC-FID and GC-MS and from CDC by ID-GC-MS. The NIST-determined values and uncertainties for omega- 3 and omega- 6 fatty acids in SRM 2378 are provided in the table below. (Note: values below are listed in $\mathrm{mg} / \mathrm{g}$, while values on the Certificate of Analysis are in units of $\mu \mathrm{g} / \mathrm{g}$.)

NIST-Determined Mass Fractions in SRM $2378(\mathrm{mg} / \mathrm{g})$

Analyte
$\alpha$-Linolenic Acid
Linoleic Acid
Arachidic Acid
EPA
DHA

$\begin{aligned} & \text { Level } 1 \\ & 0.0325 \pm 0.0041 \\ & 1.03 \pm 0.18 \\ & 0.0076 \pm 0.0011 \\ & 0.084 \pm 0.011 \\ & 0.104 \pm 0.005\end{aligned}$

$\underline{\text { Level } 2}$$$
0.0315 \pm 0.0013
$$$$
1.22 \pm 0.01
$$$$
0.0087 \pm 0.0015
$$$$
0.0207 \pm 0.008
$$$$
0.554 \pm 0.0023
$$

Human Metabolites Study Results

- Nine laboratories enrolled in this exercise and received samples to measure each of the fatty acids in human serum.

- Three laboratories reported results for $\alpha$-linolenic acid, linoleic acid, and EPA (33\% participation).

- Two laboratories reported results for DHA (22\% participation).

- One laboratory reported results for arachidic acid (11\% participation).

- The consensus ranges for all fatty acids overlapped the target ranges.

- The consensus mean for $\alpha$-linolenic acid in SRM 2378 Level 2 was below the target range (Figure 5-17).

- The consensus range for linolenic acid in SRM 2378 Level 2 was significantly larger than the target range (Figure 5-19), and the consensus mean was below the target range.

- The between-laboratory variabilities were excellent for all analytes in both matrices, at $10 \%$ or lower relative standard deviation except for linoleic acid in SRM 2378 Level 2 (17 \% RSD). Variabilities for each analyte/sample pair are reported in the table below. 


\begin{tabular}{|c|c|c|}
\hline & \multicolumn{2}{|c|}{ Between-Laboratory Variability (\% RSD) } \\
\hline Analyte & $\underline{\text { SRM } 2378 \text { Level } 1}$ & $\underline{\text { SRM } 2378 \text { Level } 2}$ \\
\hline$\alpha$-Linolenic Acid & $5 \%$ & $9 \%$ \\
\hline Linoleic Acid & $6 \%$ & $17 \%$ \\
\hline Arachidic Acid & -- & -- \\
\hline EPA & $9 \%$ & $8 \%$ \\
\hline DHA & $8 \%$ & $9 \%$ \\
\hline
\end{tabular}

- Two laboratories reported using derivatization to fatty acid methyl esters as the sample preparation method. One laboratory did not report a sample preparation method.

- Laboratories did not report the analytical method for determination of fatty acids in these samples.

Human Metabolites Technical Recommendations

The following recommendations are based on results obtained from the participants in this study. For both serum samples, too few data were reported to allow for meaningful conclusions to be drawn.

- The use of appropriate calibration materials and quality assurance samples to establish that a method is in control and performing correctly may reduce the likelihood of outlying data. Quality assurance samples can be commercially available reference materials (CRMs, SRMs, or RMs) or prepared in-house.

- A linear calibration curve which surrounds the expected sample concentration values should be used for calculations. This curve should include both the lowest and highest expected concentration values of the sample solutions. Extrapolation of results beyond calibration curves may result in incorrect values.

- In general, all results should be checked closely to avoid calculation errors and to be sure that results are reported in the requested units and in the requested form. 
Table 5-7. Individualized data summary table (NIST) for fatty acids in human serum.

\section{National Institute of Standards \& Technology}

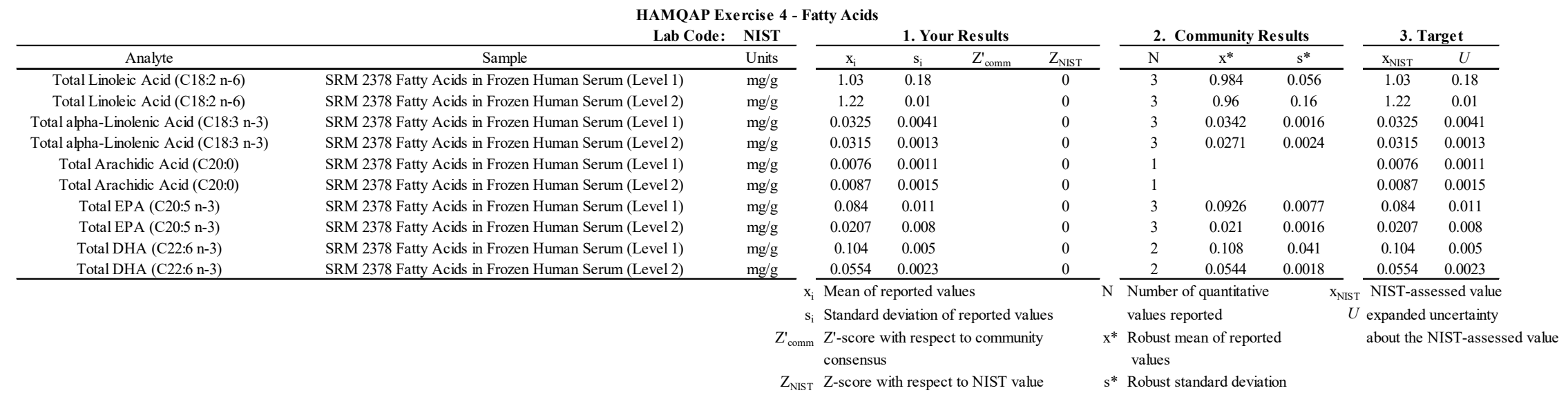


Table 5-8. Data summary table for total $\alpha$-linolenic acid in human serum.

\begin{tabular}{|c|c|c|c|c|c|c|c|c|c|c|c|}
\hline & \multirow[b]{3}{*}{ Lab } & \multicolumn{10}{|c|}{ Total alpha-Linolenic Acid (C18:3 n-3) } \\
\hline & & SRM & $\begin{array}{r}2378 \text { Fat } \\
\text { Serum }\end{array}$ & $\begin{array}{l}\text { Acids in } \\
\text { Level 1) }\end{array}$ & $\begin{array}{l}\text { Frozen } \\
(\mathrm{mg} / \mathrm{g})\end{array}$ & uman & SRM & $\begin{array}{r}2378 \text { Fat } \\
\text { Serum }\end{array}$ & $\begin{array}{l}\text { Acids in } \\
\text { Level 2) }\end{array}$ & $\begin{array}{l}\text { Frozen } 1 \\
\mathrm{mg} / \mathrm{g} \text { ) }\end{array}$ & uman \\
\hline & & $\mathbf{A}$ & B & $\mathrm{C}$ & Avg & SD & $\mathbf{A}$ & B & $\mathrm{C}$ & Avg & SD \\
\hline \multirow{10}{*}{ 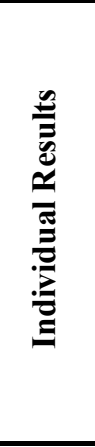 } & Target & & & & 0.0325 & 0.0041 & & & & 0.0315 & 0.0013 \\
\hline & D023 & & & & & & & & & & \\
\hline & D029 & & & & & & & & & & \\
\hline & D037 & & & & & & & & & & \\
\hline & D039 & & & & & & & & & & \\
\hline & D040 & & & & & & & & & & \\
\hline & D042 & 0.036 & 0.036 & 0.036 & 0.0360 & 0.0000 & 0.031 & 0.03 & 0.031 & 0.0307 & 0.0006 \\
\hline & D044 & 0.0316 & 0.0314 & 0.0329 & 0.0320 & 0.0008 & 0.0215 & 0.0213 & 0.022 & 0.0216 & 0.0004 \\
\hline & D052 & & & & & & & & & & \\
\hline & D054 & 0.0349 & 0.0341 & 0.0349 & 0.0346 & 0.0005 & 0.0293 & 0.0292 & 0.0289 & 0.0291 & 0.0002 \\
\hline \multirow{5}{*}{ 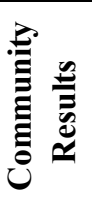 } & & \multicolumn{3}{|c|}{ Consensus Mean } & \multicolumn{2}{|l|}{0.0342} & \multicolumn{3}{|c|}{ Consensus Mean } & \multicolumn{2}{|l|}{0.0271} \\
\hline & & \multicolumn{3}{|c|}{ Consensus Standard Deviation } & \multirow{2}{*}{\multicolumn{2}{|c|}{$\begin{array}{l}0.0016 \\
0.0360\end{array}$}} & \multirow{2}{*}{\multicolumn{3}{|c|}{ Consensus Standard Deviation }} & \multicolumn{2}{|l|}{0.0024} \\
\hline & & & & & & & & \multicolumn{2}{|l|}{0.0307} \\
\hline & & \multicolumn{3}{|c|}{ Minimum } & $\begin{array}{l}0.0360 \\
0.0320\end{array}$ & 0.0320 & \multicolumn{2}{|c|}{ Maximum } & Minimum & \multicolumn{2}{|l|}{0.0216} \\
\hline & & \multicolumn{3}{|l|}{$\mathrm{N}$} & & \multicolumn{3}{|c|}{$\mathrm{N}$} & \\
\hline
\end{tabular}




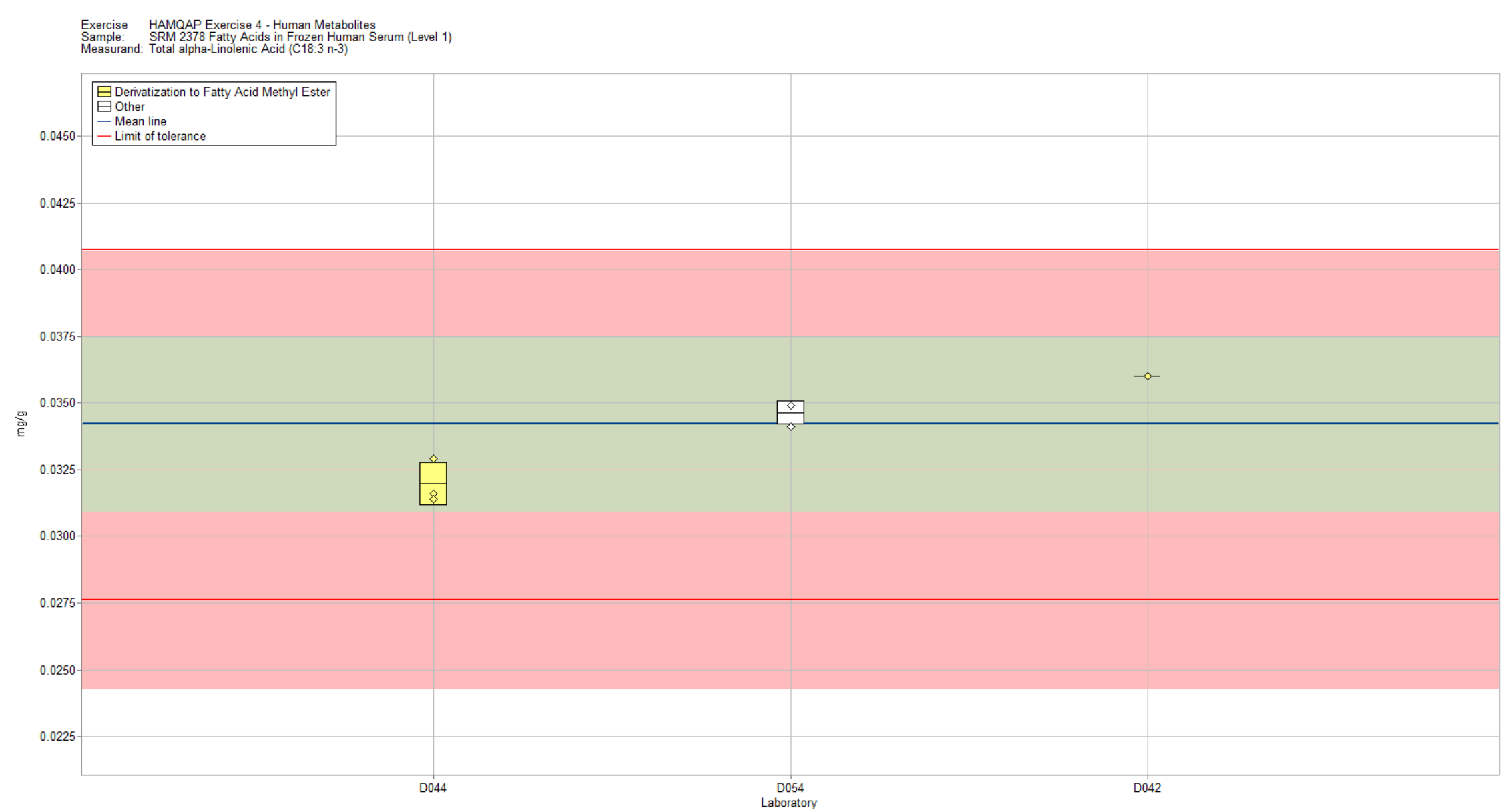

Figure 5-16. Total $\alpha$-linolenic acid in SRM 2378 Fatty Acids in Frozen Human Serum (Level 1) (data summary view - sample preparation method). In this view, individual laboratory data are plotted (diamonds) with the individual laboratory standard deviation (rectangle). The color of the data point represents the sample preparation method employed. The solid blue line represents the consensus mean, and the green shaded region represents the $95 \%$ confidence interval for the consensus mean. The red solid lines represent the consensus range of tolerance, calculated as the values above and below the consensus mean that result in an acceptable $Z_{\text {comm }}^{\prime}$ score, $\left|Z_{\text {comm }}^{\prime}\right| \leq 2$. The red shaded region represents the NIST range of tolerance, which encompasses the target value bounded by its uncertainty $\left(U_{\mathrm{NIST}}\right)$ and represents the range that results in an acceptable $Z_{\mathrm{NIST}}$ score, $\left|Z_{\mathrm{NIST}}\right| \leq 2$. 


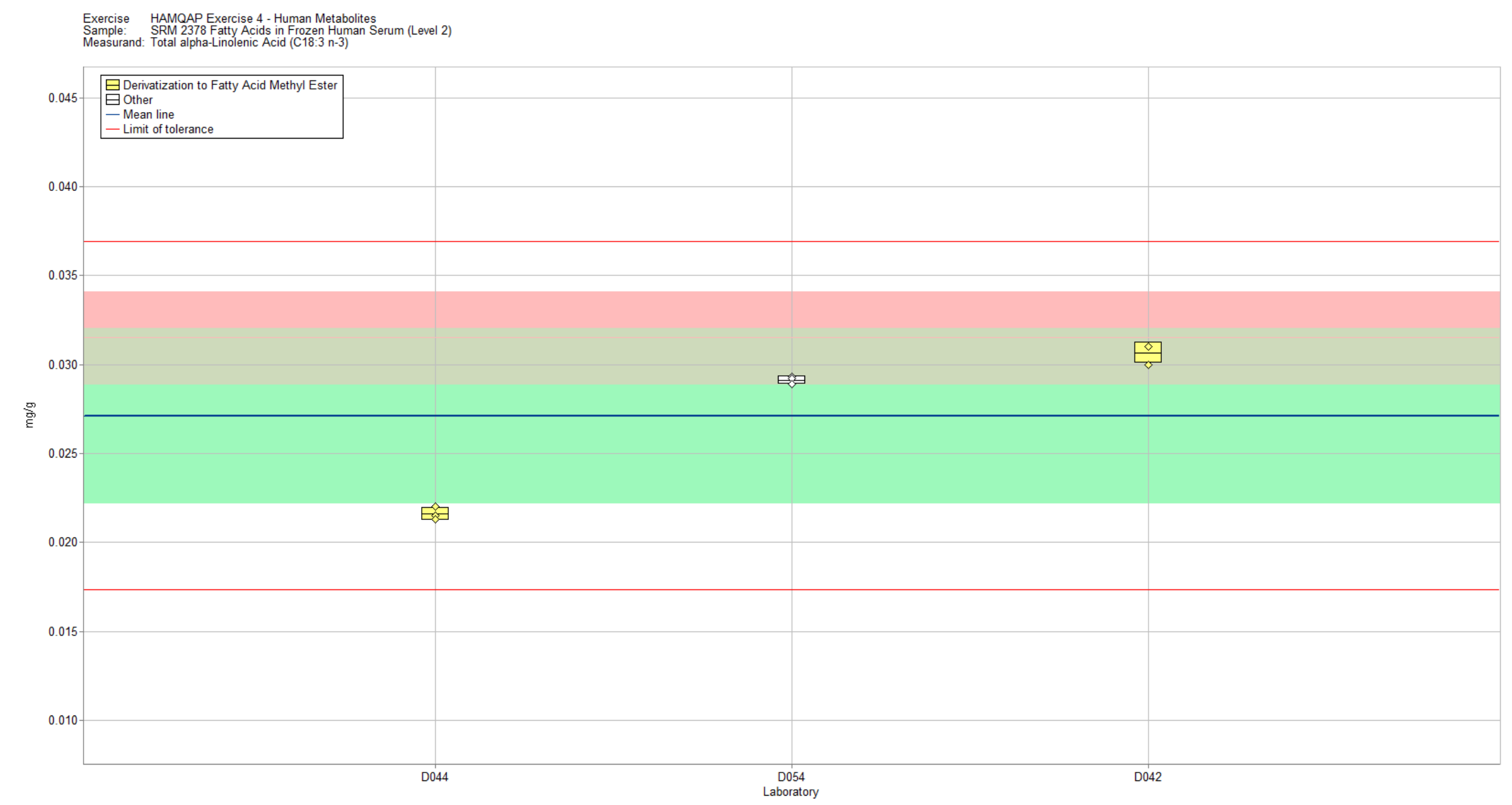

Figure 5-17. Total $\alpha$-linolenic acid in SRM 2378 Fatty Acids in Frozen Human Serum (Level 2) (data summary view - sample preparation method). In this view, individual laboratory data are plotted (diamonds) with the individual laboratory standard deviation (rectangle). The color of the data point represents the sample preparation method employed. The solid blue line represents the consensus mean, and the green shaded region represents the $95 \%$ confidence interval for the consensus mean. The red solid lines represent the consensus range of tolerance, calculated as the values above and below the consensus mean that result in an acceptable $Z_{\text {comm }}^{\prime}$ score, $\left|Z_{\text {comm }}^{\prime}\right| \leq 2$. The red shaded region represents the NIST range of tolerance, which encompasses the target value bounded by its uncertainty $\left(U_{\text {NIST }}\right)$ and represents the range that results in an acceptable $Z_{\text {NIST }}$ score, $\left|Z_{\text {NIST }}\right| \leq 2$. 
Table 5-9. Data summary table for total linoleic acid in human serum.

\begin{tabular}{|c|c|c|c|c|c|c|c|c|c|c|c|}
\hline & \multirow[b]{3}{*}{ Lab } & \multicolumn{10}{|c|}{ Total Linoleic Acid (C18:2 n-6) } \\
\hline & & SRM & $\begin{array}{r}378 \mathrm{Fa} \\
\text { Serun }\end{array}$ & $\begin{array}{l}\text { Acids in } \\
\text { Level 1) }\end{array}$ & $\begin{array}{l}\text { rozen } \\
\mathrm{ng} / \mathrm{g})\end{array}$ & $\operatorname{man}$ & SRI & $\begin{array}{r}378 \mathrm{Fa} \\
\text { Serun }\end{array}$ & $\begin{array}{l}\text { Acids in } \\
\text { Level 2) }\end{array}$ & $\begin{array}{l}\text { Frozen } \\
\mathrm{mg} / \mathrm{g})\end{array}$ & $\operatorname{man}$ \\
\hline & & $\mathbf{A}$ & $\mathbf{B}$ & $\mathrm{C}$ & Avg & SD & $\mathbf{A}$ & $\mathbf{B}$ & $\mathbf{C}$ & Avg & SD \\
\hline \multirow{10}{*}{ 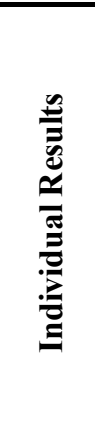 } & Target & & & & 1.03 & 0.18 & & & & 1.220 & 0.010 \\
\hline & D023 & & & & & & & & & & \\
\hline & D029 & & & & & & & & & & \\
\hline & D037 & & & & & & & & & & \\
\hline & D039 & & & & & & & & & & \\
\hline & D040 & & & & & & & & & & \\
\hline & D042 & 1.05 & 1.02 & 1.04 & 1.04 & 0.02 & 1.12 & 1.1 & 1.12 & 1.113 & 0.012 \\
\hline & D044 & 0.927 & 0.932 & 0.937 & 0.93 & 0.01 & 0.78 & 0.782 & 0.787 & 0.783 & 0.004 \\
\hline & D052 & & & & & & & & & & \\
\hline & D054 & 1 & 0.971 & 0.975 & 0.98 & 0.02 & 1 & 1.01 & 0.981 & 0.997 & 0.015 \\
\hline \multirow{5}{*}{ 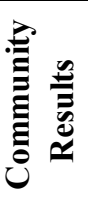 } & & \multicolumn{3}{|c|}{ Consensus Mean } & \multicolumn{2}{|l|}{0.98} & \multicolumn{3}{|c|}{ Consensus Mean } & 0.964 & \\
\hline & & \multicolumn{3}{|c|}{ Consensus Standard Deviation } & \multicolumn{2}{|l|}{0.06} & \multicolumn{3}{|c|}{ Consensus Standard Deviation } & 0.163 & \\
\hline & & \multicolumn{3}{|c|}{ Maximum } & \multicolumn{2}{|l|}{1.04} & \multicolumn{3}{|c|}{ Maximum } & 1.113 & \\
\hline & & \multicolumn{3}{|c|}{ Minimum } & \multicolumn{2}{|l|}{0.93} & \multicolumn{3}{|c|}{ Minimum } & 0.783 & \\
\hline & & \multicolumn{3}{|c|}{$\mathrm{N}$} & \multicolumn{2}{|l|}{3} & \multicolumn{3}{|c|}{$\mathrm{N}$} & 3 & \\
\hline
\end{tabular}




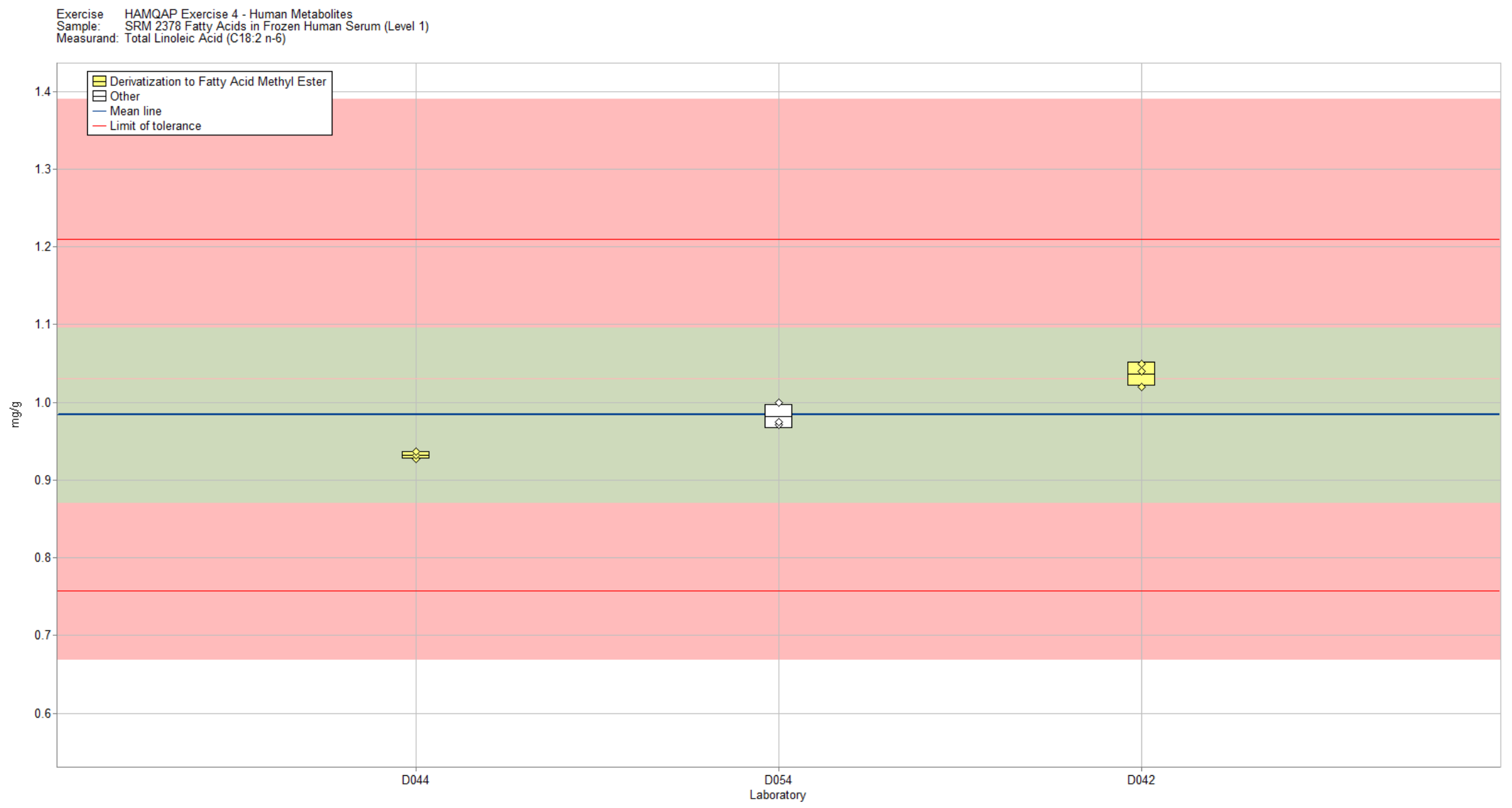

Figure 5-18. Total linoleic acid in SRM 2378 Fatty Acids in Frozen Human Serum (Level 1) (data summary view - sample preparation method). In this view, individual laboratory data are plotted (diamonds) with the individual laboratory standard deviation (rectangle). The color of the data point represents the sample preparation method employed. The solid blue line represents the consensus mean, and the green shaded region represents the $95 \%$ confidence interval for the consensus mean. The red solid lines represent the consensus range of tolerance, calculated as the values above and below the consensus mean that result in an acceptable $Z_{\text {comm }}^{\prime}$ score, $\left|Z_{\text {comm }}^{\prime}\right| \leq 2$. The red shaded region represents the NIST range of tolerance, which encompasses the target value bounded by its uncertainty ( $\left.U_{\mathrm{NIST}}\right)$ and represents the range that results in an acceptable $Z_{\text {NIST }}$ score, $\left|Z_{\mathrm{NIST}}\right| \leq 2$. 


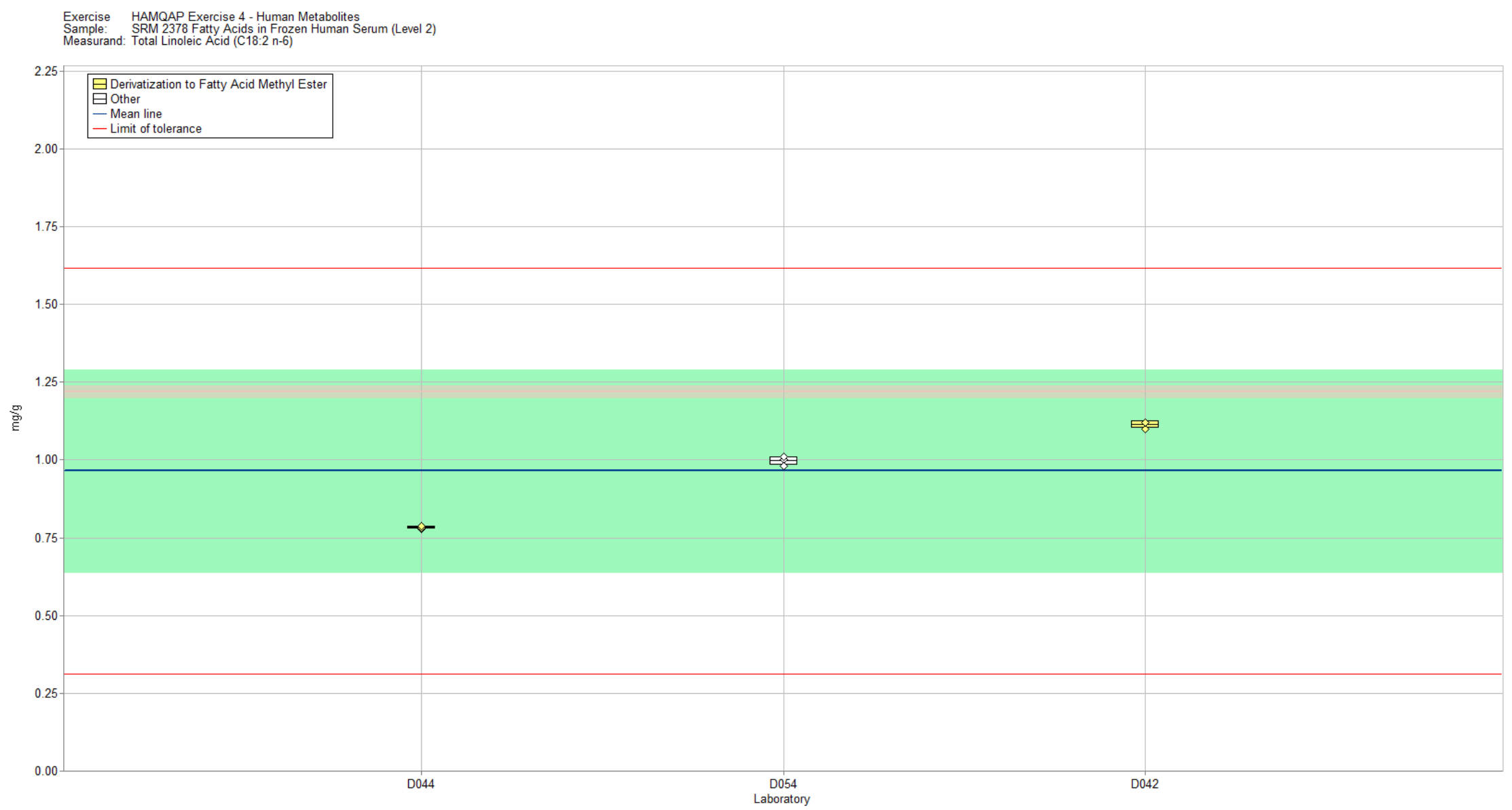

Figure 5-19. Total linoleic acid in SRM 2378 Fatty Acids in Frozen Human Serum (Level 2) (data summary view - sample preparation method). In this view, individual laboratory data are plotted (diamonds) with the individual laboratory standard deviation (rectangle). The color of the data point represents the sample preparation method employed. The solid blue line represents the consensus mean, and the green shaded region represents the $95 \%$ confidence interval for the consensus mean. The red solid lines represent the consensus range of tolerance, calculated as the values above and below the consensus mean that result in an acceptable $Z_{\text {comm }}^{\prime}$ score, $\left|Z_{\text {comm }}^{\prime}\right| \leq 2$. The red shaded region represents the NIST range of tolerance, which encompasses the target value bounded by its uncertainty $\left(U_{\text {NIST }}\right)$ and represents the range that results in an acceptable $Z_{\text {NIST }}$ score, $\left|Z_{\text {NIST }}\right| \leq 2$. 
Table 5-10. Data summary table for total arachidic acid in human serum.

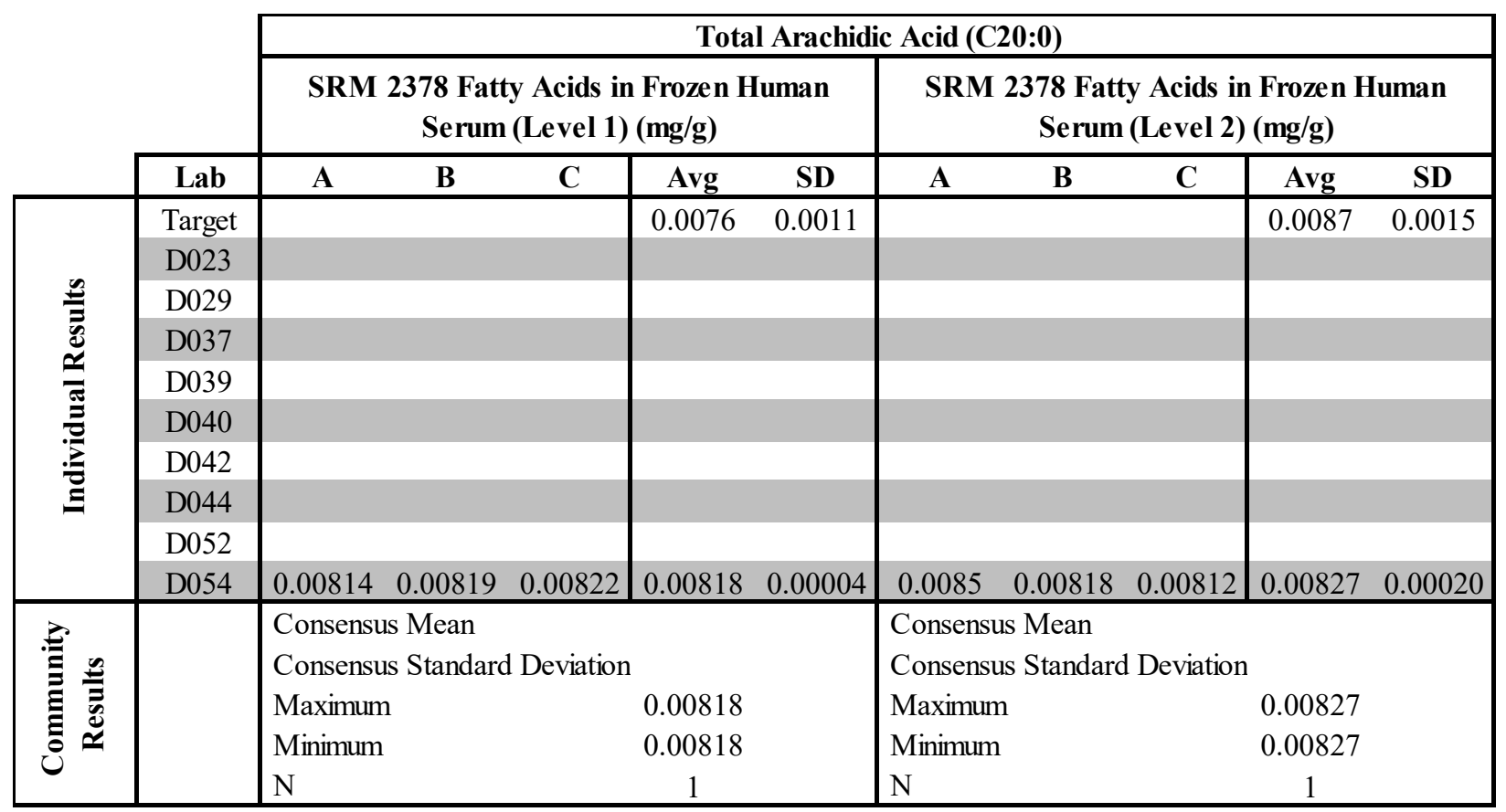


Table 5-11. Data summary table for total EPA in human serum.

\begin{tabular}{|c|c|c|c|c|c|c|c|c|c|c|c|}
\hline & \multirow[b]{3}{*}{ Lab } & \multicolumn{10}{|c|}{ Total EPA (C20:5 n-3) } \\
\hline & & SRI & $\begin{array}{r}2378 \text { Fat } \\
\text { Serun }\end{array}$ & $\begin{array}{l}\text { Acids in } \\
\text { Level 1) }\end{array}$ & $\begin{array}{l}\text { Frozen } \\
\mathrm{mg} / \mathrm{g})\end{array}$ & man & SRM & $\begin{array}{r}2378 \text { Fat } \\
\text { Serun }\end{array}$ & $\begin{array}{l}\text { Acids in } \\
\text { Level 2) }\end{array}$ & $\begin{array}{l}\text { Frozen H } \\
(\mathrm{mg} / \mathrm{g})\end{array}$ & uman \\
\hline & & $\mathbf{A}$ & $\mathbf{B}$ & $\mathrm{C}$ & Avg & SD & $\mathbf{A}$ & $\mathbf{B}$ & $\mathbf{C}$ & Avg & SD \\
\hline \multirow{10}{*}{ 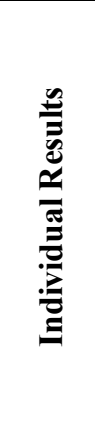 } & Target & & & & 0.084 & 0.011 & & & & 0.0207 & 0.0080 \\
\hline & D023 & & & & & & & & & & \\
\hline & D029 & & & & & & & & & & \\
\hline & D037 & & & & & & & & & & \\
\hline & D039 & & & & & & & & & & \\
\hline & D040 & & & & & & & & & & \\
\hline & D042 & 0.1 & 0.099 & 0.1 & 0.100 & 0.001 & 0.022 & 0.023 & 0.023 & 0.02267 & 0.00058 \\
\hline & D044 & 0.0854 & 0.0856 & 0.0861 & 0.086 & 0.000 & 0.0205 & 0.0208 & 0.021 & 0.02077 & 0.00025 \\
\hline & D052 & & & & & & & & & & \\
\hline & D054 & 0.0954 & 0.0902 & 0.092 & 0.093 & 0.003 & 0.0196 & 0.0198 & 0.0194 & 0.01960 & 0.00020 \\
\hline \multirow{5}{*}{ 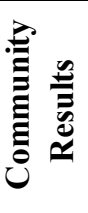 } & & \multicolumn{3}{|c|}{ Consensus Mean } & \multicolumn{2}{|l|}{0.093} & \multicolumn{4}{|c|}{$\begin{array}{lc}\text { Consensus Mean } & 0.02101\end{array}$} & \\
\hline & & \multicolumn{3}{|c|}{ Consensus Standard Deviation } & \multicolumn{2}{|c|}{0.008} & \multicolumn{5}{|c|}{ Consensus Standard Deviation 0.00156} \\
\hline & & \multicolumn{3}{|c|}{ Maximum } & \multicolumn{2}{|l|}{0.100} & \multicolumn{3}{|c|}{ Maximum } & \multicolumn{2}{|l|}{0.02267} \\
\hline & & \multirow{2}{*}{\multicolumn{3}{|c|}{ Minimum }} & \multicolumn{2}{|c|}{0.086} & \multicolumn{3}{|c|}{ Minimum } & \multicolumn{2}{|l|}{0.01960} \\
\hline & & & & & 3 & & & & & 3 & \\
\hline
\end{tabular}


Exercise HAMQAP Exercise 4- Human Metabolites
Sample:
Measurand: Total 238 F FAtcy Acids in Frozen Human Serum (Level 1)
(C20:5 $\mathrm{n}-3$ )

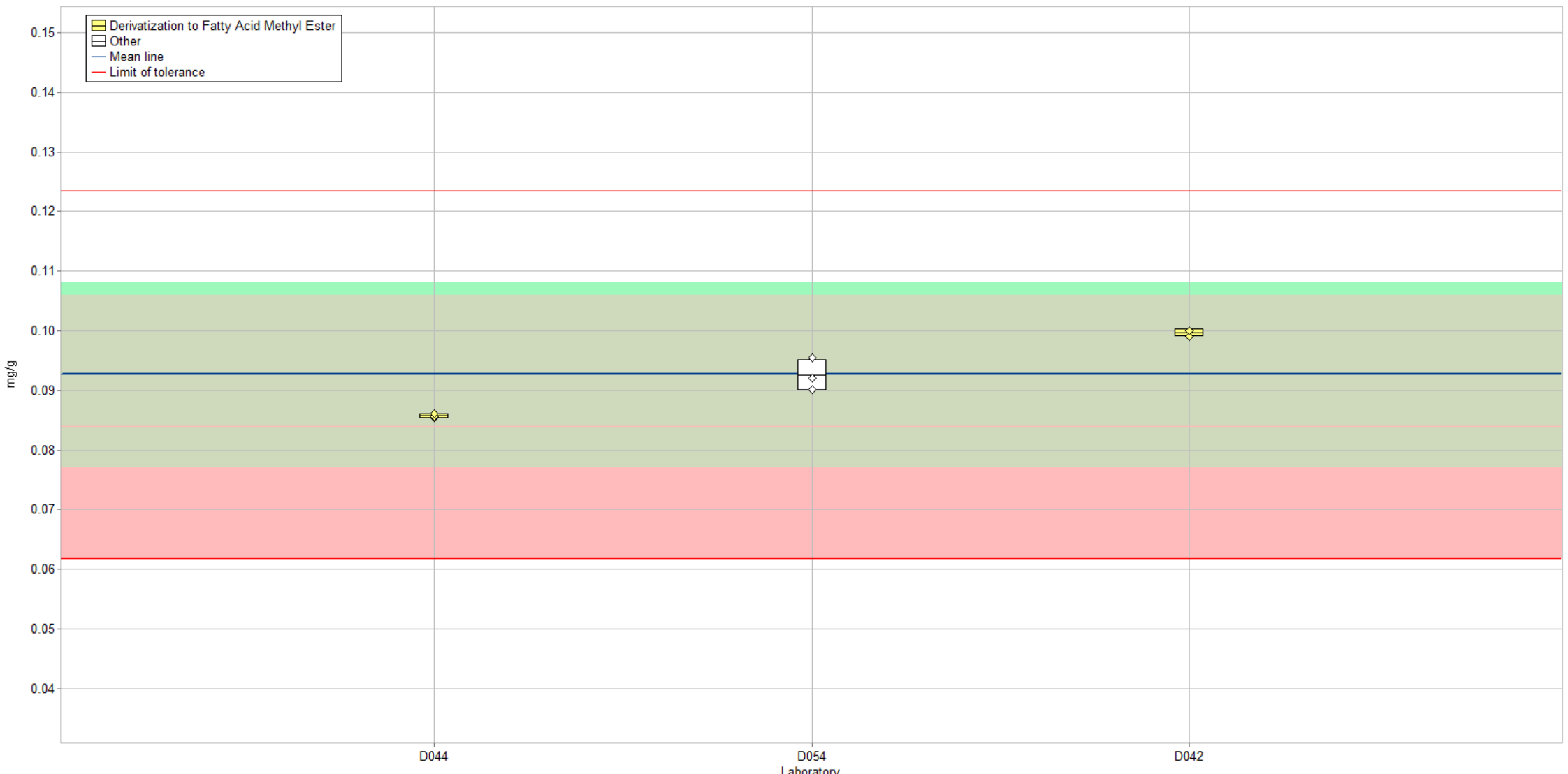

Figure 5-20. Total EPA in SRM 2378 Fatty Acids in Frozen Human Serum (Level 1) (data summary view - sample preparation method). In this view, individual laboratory data are plotted (diamonds) with the individual laboratory standard deviation (rectangle). The color of the data point represents the sample preparation method employed. The solid blue line represents the consensus mean, and the green shaded region represents the $95 \%$ confidence interval for the consensus mean. The red solid lines represent the consensus range of tolerance, calculated as the values above and below the consensus mean that result in an acceptable $Z_{\text {comm }}^{\prime}$ score, $\left|Z_{\text {comm }}^{\prime}\right| \leq 2$. The red shaded region represents the NIST range of tolerance, which encompasses the target value bounded by its uncertainty ( $\left.U_{\text {NIST }}\right)$ and represents the range that results in an acceptable $Z_{\text {NIST }}$ score, $\left|Z_{\mathrm{NIST}}\right| \leq 2$. 


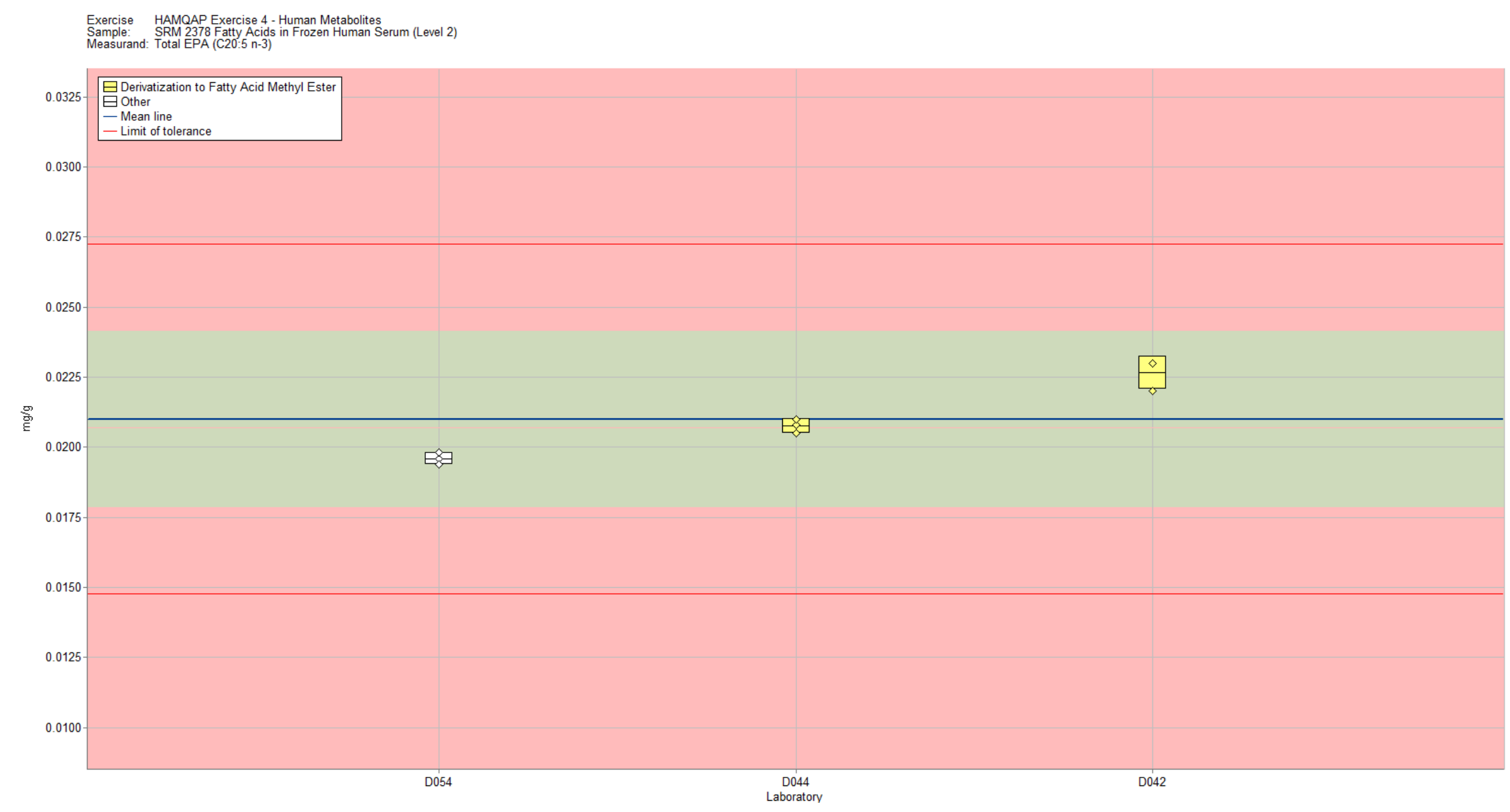

Figure 5-21. Total EPA in SRM 2378 Fatty Acids in Frozen Human Serum (Level 2) (data summary view - sample preparation method). In this view, individual laboratory data are plotted (diamonds) with the individual laboratory standard deviation (rectangle). The color of the data point represents the sample preparation method employed. The solid blue line represents the consensus mean, and the green shaded region represents the $95 \%$ confidence interval for the consensus mean. The red solid lines represent the consensus range of tolerance, calculated as the values above and below the consensus mean that result in an acceptable $Z_{\text {comm }}^{\prime}$ score, $\left|Z_{\text {comm }}^{\prime}\right| \leq 2$. The red shaded region represents the NIST range of tolerance, which encompasses the target value bounded by its uncertainty ( $\left.U_{\mathrm{NIST}}\right)$ and represents the range that results in an acceptable $Z_{\text {NIST }}$ score, $\left|Z_{\mathrm{NIST}}\right| \leq 2$. 
Table 5-12. Data summary table for total DHA in human serum.

\begin{tabular}{|c|c|c|c|c|c|c|c|c|c|c|c|}
\hline & \multirow[b]{3}{*}{ Lab } & \multicolumn{10}{|c|}{ Total DHA (C22:6 n-3) } \\
\hline & & \multicolumn{5}{|c|}{$\begin{array}{c}\text { SRM } 2378 \text { Fatty Acids in Frozen Human } \\
\text { Serum (Level 1) (mg/g) }\end{array}$} & \multicolumn{5}{|c|}{$\begin{array}{l}\text { SRM } 2378 \text { Fatty Acids in Frozen Human } \\
\text { Serum (Level 2) }(\mathrm{mg} / \mathrm{g})\end{array}$} \\
\hline & & $\mathbf{A}$ & $\mathbf{B}$ & $\mathrm{C}$ & Avg & SD & $\mathbf{A}$ & $\mathbf{B}$ & $\mathbf{C}$ & Avg & SD \\
\hline \multirow{10}{*}{ 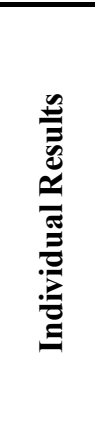 } & Target & & & & 0.1040 & 0.0050 & & & & 0.0554 & 0.0023 \\
\hline & D023 & & & & & & & & & & \\
\hline & D029 & & & & & & & & & & \\
\hline & D037 & & & & & & & & & & \\
\hline & D039 & & & & & & & & & & \\
\hline & D040 & & & & & & & & & & \\
\hline & D042 & & & & & & & & & & \\
\hline & D044 & 0.0936 & 0.094 & 0.0945 & 0.0940 & 0.0005 & 0.0548 & 0.055 & 0.0567 & 0.0555 & 0.0010 \\
\hline & D052 & & & & & & & & & & \\
\hline & D054 & 0.126 & 0.122 & 0.12 & 0.1227 & 0.0031 & 0.055 & 0.0528 & 0.0524 & 0.0534 & 0.0014 \\
\hline \multirow{5}{*}{ 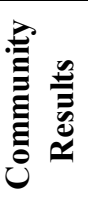 } & & \multicolumn{3}{|c|}{ Consensus Mean } & \multicolumn{2}{|l|}{0.1084} & \multicolumn{2}{|c|}{ Consensus Mean } & & 0.0545 & \\
\hline & & \multicolumn{3}{|c|}{ Consensus Standard Deviation } & \multicolumn{2}{|l|}{0.0413} & \multicolumn{3}{|c|}{ Consensus Standard Deviation } & 0.0019 & \\
\hline & & \multicolumn{3}{|c|}{ Maximum } & \multicolumn{2}{|l|}{0.1227} & \multicolumn{3}{|c|}{ Maximum } & 0.0555 & \\
\hline & & \multicolumn{3}{|c|}{ Minimum } & \multicolumn{2}{|l|}{0.0940} & & 0.0534 & \\
\hline & & \multicolumn{3}{|c|}{$\mathrm{N}$} & \multicolumn{2}{|l|}{$\begin{array}{c}0.0940 \\
2\end{array}$} & \multicolumn{3}{|c|}{$\mathrm{N}$} & 2 & \\
\hline
\end{tabular}




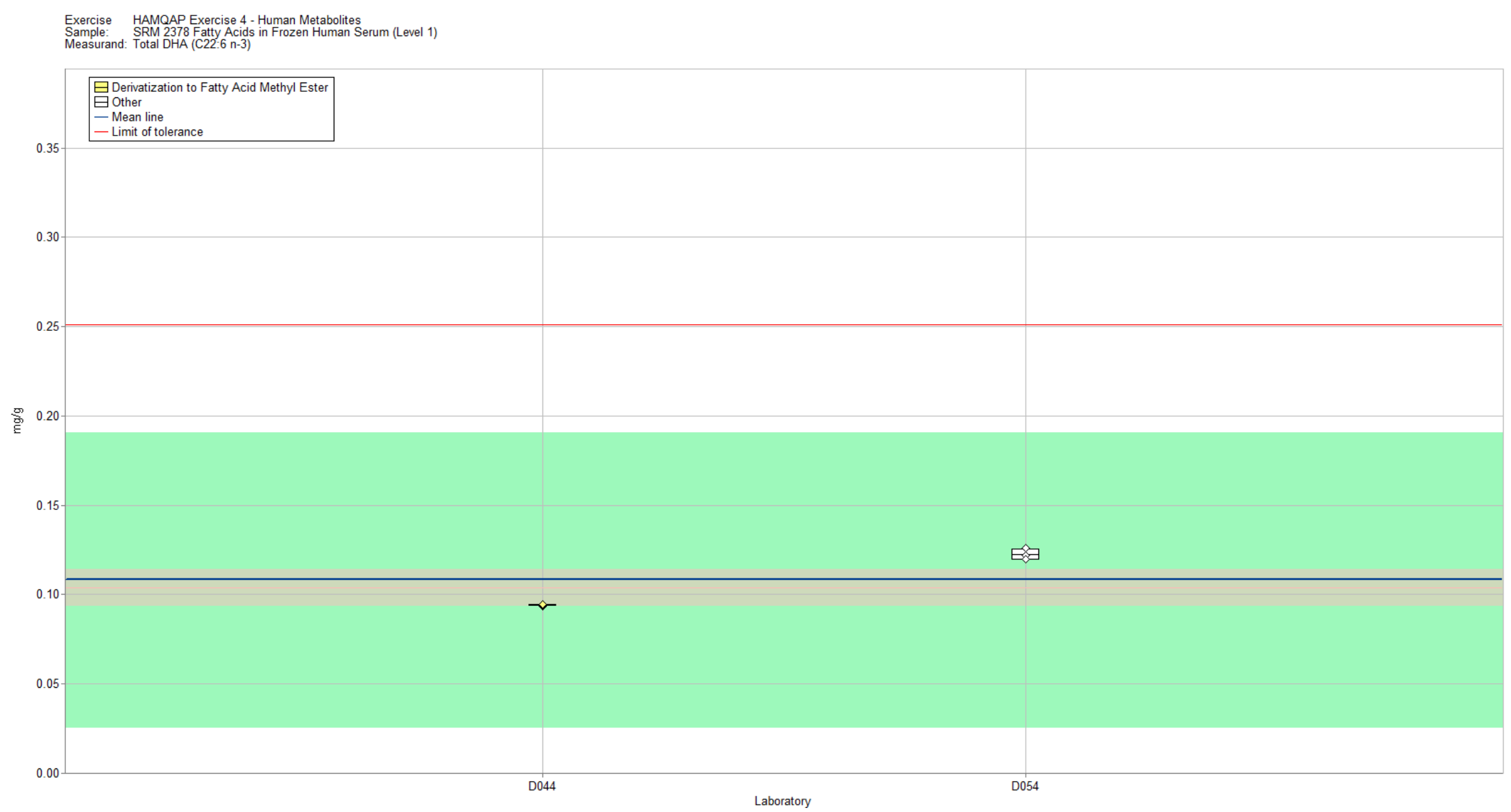

Figure 5-22. Total DHA in SRM 2378 Fatty Acids in Frozen Human Serum (Level 1) (data summary view - sample preparation method). In this view, individual laboratory data are plotted (diamonds) with the individual laboratory standard deviation (rectangle). The color of the data point represents the sample preparation method employed. The solid blue line represents the consensus mean, and the green shaded region represents the $95 \%$ confidence interval for the consensus mean. The solid red line represents the upper consensus range of tolerance, calculated as the values above the consensus mean that result in an acceptable $Z_{\text {comm }}^{\prime}$ score, $\left|Z_{\text {comm }}^{\prime}\right| \leq 2$, with the lower limit set at zero. The red shaded region represents the NIST range of tolerance, which encompasses the target value bounded by its uncertainty ( $U_{\text {NIST }}$ ) and represents the range that results in an acceptable $Z_{\text {NIST }}$ score, $\left|Z_{\text {NIST }}\right| \leq 2$. 


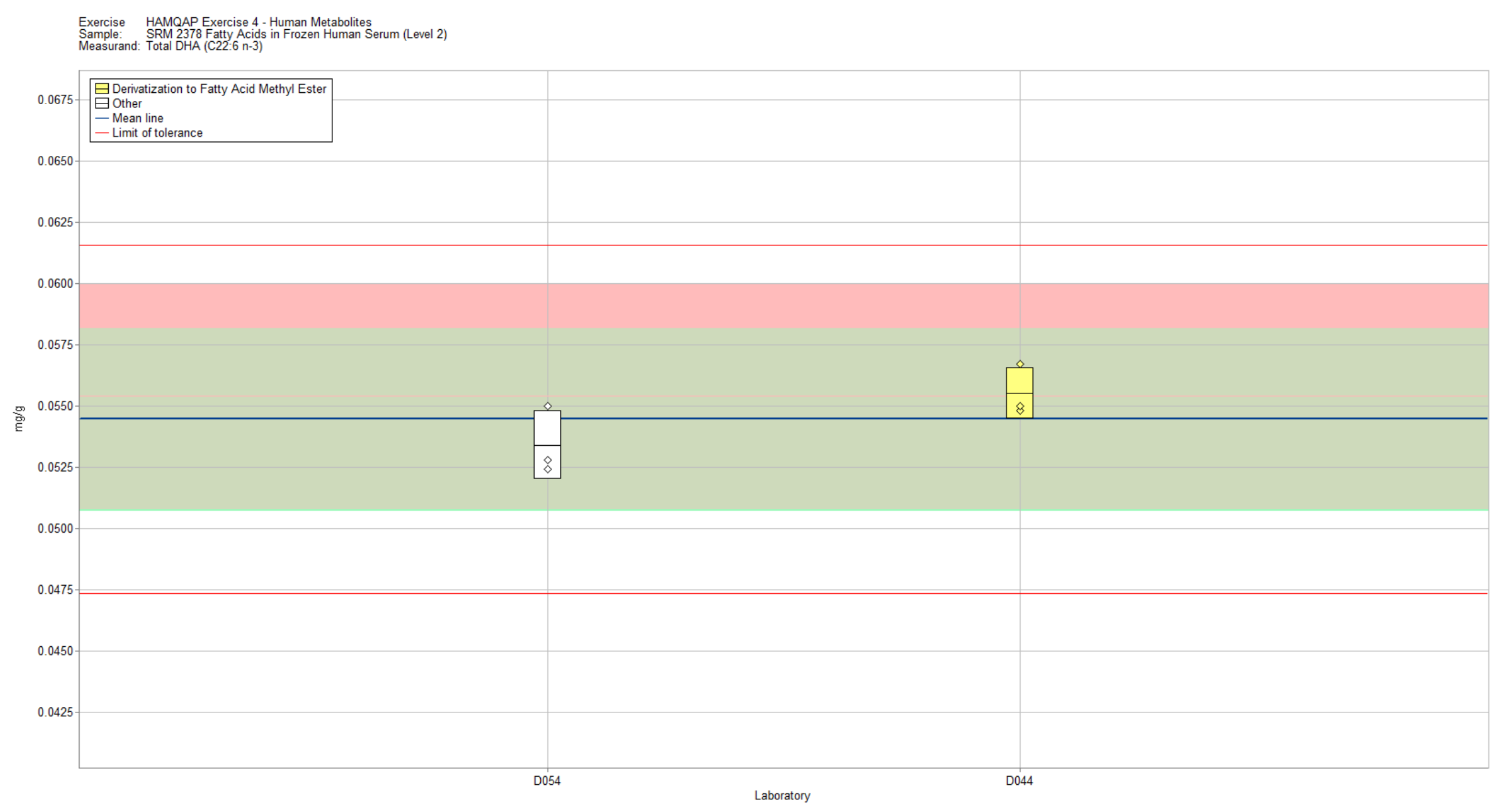

Figure 5-23. Total DHA in SRM 2378 Fatty Acids in Frozen Human Serum (Level 2) (data summary view - sample preparation method). In this view, individual laboratory data are plotted (diamonds) with the individual laboratory standard deviation (rectangle). The color of the data point represents the sample preparation method employed. The solid blue line represents the consensus mean, and the green shaded region represents the $95 \%$ confidence interval for the consensus mean. The red solid lines represent the consensus range of tolerance, calculated as the values above and below the consensus mean that result in an acceptable $Z_{\text {comm }}^{\prime}$ score, $\left|Z_{\text {comm }}^{\prime}\right| \leq 2$. The red shaded region represents the NIST range of tolerance, which encompasses the target value bounded by its uncertainty ( $\left.U_{\text {NIST }}\right)$ and represents the range that results in an acceptable $Z_{\text {NIST }}$ score, $\left|Z_{\mathrm{NIST}}\right| \leq 2$. 
Fatty Acids Overall Study Comparison

Overall, laboratories measuring fatty acids in fish oils and serum were successful based on the limited results reported.

- A few laboratories reported data outside of the target ranges for the fish oil samples, but overall results were excellent.

- Clinical laboratories had lower participation, but those laboratories reporting results were in good agreement. The limited number of participating laboratories could indicate the measurement is challenging or limited interest exists in the clinical community. 


\section{SECTION 6: BOTANICALS (Phenolics)}

\section{$\underline{\text { Study Overview }}$}

In this study, participants were provided with samples of SRM 3262 St. John's Wort (Hypericum perforatum L.) Aerial Parts and St. John's Wort (Hypericum perforatum L.) Tablets. Participants were asked to use in-house analytical methods to determine the mass fraction $(\mathrm{mg} / \mathrm{g})$ of select phenolics (hyperoside, pseudohypericin, hyperforin, adhyperforin, quercetin, quercitrin, isoquercetin, rutin, chlorogenic acid) in each matrix. St. John's Wort (Hypericum perforatum L.) is often used as a botanical supplement to combat mild to moderate depression, although efficacy studies report mixed results. ${ }^{14}$ Contradictory findings may result if researchers have not verified the authenticity or characterized the chemical composition of the intervention materials used in clinical studies. Without a comprehensive understanding of the intervention materials, correlations between treatment and clinical improvements or side effects are unreliable.

\section{Dietary Intake Sample Information}

St. John's Wort Aerial Parts. Participants were provided with three packets, each containing $3.3 \mathrm{~g}$ of powdered St. John's Wort. Participants were asked to store the material at controlled room temperature, $20{ }^{\circ} \mathrm{C}$ to $25{ }^{\circ} \mathrm{C}$, and to prepare one sample and report one value from each packet provided. Before use, participants were instructed to mix the contents of the packet thoroughly, and to use a sample size at least $100 \mathrm{mg}$. The approximate analyte levels were not reported to participants prior to the study. The reference values for hyperoside, pseudohypericin, quercitrin, rutin, and chlorogenic acid in SRM 3262 were assigned using results from NIST by LC-absorbance and LC-fluorescence. The reference values and uncertainties are provided in the table below, both on a dry-mass basis, as shown on the COA, and on an as-received basis accounting for moisture of the material (4.9\%). Target values for hyperforin, adhyperforin, quercetin, and isoquercetin in SRM 3262 have not been determined.

\section{NIST-Determined Mass Fraction in SRM $3262(\mathrm{mg} / \mathrm{g})$}

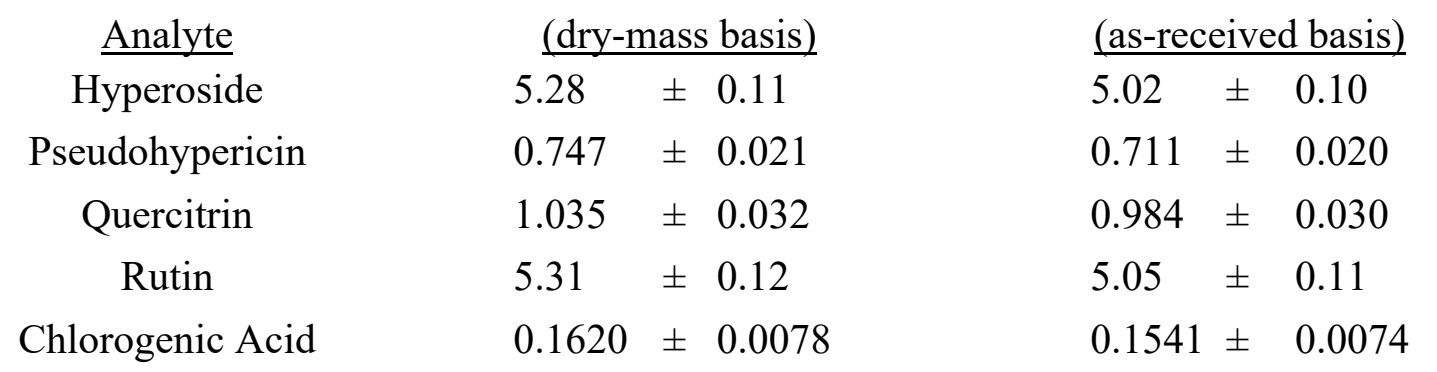

St. John's Wort Tablets. Participants were provided with three packets, each containing 10 tablets of St. John's Wort. Participants were asked to store the material at controlled room temperature, $20^{\circ} \mathrm{C}$ to $25^{\circ} \mathrm{C}$, to use a sample size appropriate for their in-house method of analysis, and to prepare one sample and report one value from each packet provided. Before use, participants were instructed to grind all 10 tablets and to mix the resulting powder thoroughly. After grinding, the resulting powder can be stored at $-20^{\circ} \mathrm{C}$ and should be analyzed within 2 days. Participants were asked to prepare three samples and report three values from each packet provided. The

\footnotetext{
${ }^{14}$ St. John's Wort: At a Glance. National Institutes of Health National Center for Complementary and Integrative Health. https://nccih.nih.gov/health/stjohnswort/ataglance.htm (accessed March 2020).
} 
approximate analyte levels were not reported to participants prior to the study, and target values in this material have not been determined.

Dietary Intake Study Results

- Nineteen laboratories enrolled in this exercise and received samples to measure some or all of the phenolics in St. John's Wort aerial parts and tablets. The enrollment and reporting statistics for the botanicals study is described in the table below. Some of the reported values were nonquantitative (zero or below LOQ) but are included in the participation and reporting statistics.

\begin{tabular}{|c|c|c|c|}
\hline \multirow[b]{2}{*}{ Analyte } & \multirow{2}{*}{$\begin{array}{c}\frac{\text { Number of }}{\text { Laboratories }} \\
\underline{\text { Requesting Samples }}\end{array}$} & \multicolumn{2}{|c|}{$\frac{\text { Number of Laboratories Reporting Resul }}{\text { (Percent Participation) }}$} \\
\hline & & Aerial Parts & $\underline{\text { Tablets }}$ \\
\hline Hyperoside & 12 & $1(12 \%)$ & $2(17 \%)$ \\
\hline Pseudohypericin & 12 & $3(25 \%)$ & $4(33 \%)$ \\
\hline Quercitrin & 11 & $3(27 \%)$ & $3(27 \%)$ \\
\hline Rutin & 16 & $6(38 \%)$ & $7(44 \%)$ \\
\hline Chlorogenic Acid & 16 & $6(38 \%)$ & $8(50 \%)$ \\
\hline Adhyperforin & 10 & $1(10 \%)$ & $1(10 \%)$ \\
\hline Hyperforin & 9 & $1(11 \%)$ & $3(33 \%)$ \\
\hline Isoquercetin & 12 & $2(17 \%)$ & $2(17 \%)$ \\
\hline Quercetin & 19 & $7(42 \%)$ & $8(47 \%)$ \\
\hline
\end{tabular}

- The between-laboratory variabilities were acceptable for most analytes in the St. John's Wort aerial parts and for rutin, chlorogenic acid, and quercetin in the St. John's Wort tablets (see table below). Variabilities for other analytes were either very large ( $>85 \%$ RSD) or unable to be determined based on a limited number of quantitative results reported.

\begin{tabular}{ccc} 
& \multicolumn{2}{c}{ Between-Laboratory Variability (\% RSD) } \\
\cline { 2 - 3 } Analyte & Aerial Parts & $\frac{\text { Tablets }}{85 \%}$ \\
Hyperoside & -- & $89 \%$ \\
Pseudohypericin & $24 \%$ & $>100 \%$ \\
Quercitrin & $22 \%$ & $7 \%$ \\
Rutin & $24 \%$ & $10 \%$ \\
Chlorogenic Acid & $15 \%$ & -- \\
Adhyperforin & -- & $>100 \%$ \\
Hyperforin & -- & $>100 \%$ \\
Isoquercetin & $100 \%$ & $17 \%$ \\
Quercetin & $23 \%$ &
\end{tabular}


- For St. John's Wort aerial parts, the consensus means for pseudohypericin and quercitrin (Figures 6-2 and 6-4) were below the NIST target range. The consensus mean for rutin (Figure 6-6) was slightly below the NIST target range but the consensus range encompassed the NIST target range. The consensus mean for chlorogenic acid (Figure 6-8) was above the NIST target range.

- All participating laboratories reported using LC-absorbance for determination of the phenolics in the St. John's Wort samples. One laboratory did not report an analytical method for quercitrin (Figures 6-1 to 6-16).

- Most laboratories reported using solvent extraction for determination of the phenolics in the St. John's Wort samples. Additionally, one laboratory reported using dilution and one reported other.

\section{Dietary Intake Technical Recommendations}

The following recommendations and observations are based on results obtained from the participants in this study.

- Despite a relatively large number of laboratories requesting samples for this study, overall participation was low and limits the ability to make technical recommendations.

- Laboratories reported results for common flavonols (rutin, chlorogenic acid, quercitin), but limited results were received for analytes specific to St. John's Wort (naphthodianthrones, phloroglucinols).

- Low participation may be the result of difficulty with St. John's Wort sample preparation and analysis, leading laboratories to withhold results.

- Challenges in sample preparation may have resulted in results that were lower than the target value or high variability within or between laboratories.

- Laboratories reporting results below the target value or large sample-to-sample variability should examine sample preparation conditions. Complete extraction of these analytes from the botanical matrices may require use of less common solvents or multiple extraction cycles.

- Any extraction procedure should be optimized to determine the most effective extraction solvent and to ensure exhaustive extraction of the analyte from the matrix.

- The optimum number of extraction cycles must be determined by sequential reextraction of the sample matrix until no further increase in yield is observed. Sequential extractions may be needed if the extraction solvent becomes saturated during the first (or only) extraction cycle.

- The St. John's Wort tablets should require a less intensive extraction procedure than the aerial parts, but botanical tablets can be difficult to grind and homogenize into a uniform material, resulting into large within- or between-laboratory variability.

- Improper calibration is a frequent source of measurement error.

- Calibrant purity is an important consideration in analytical measurements. Where possible, calibrants should be evaluated for purity and presence of residual solvents prior to use. The measured purity should be used to correct the concentrations of the solutions used for calibration. Because synthesis of calibration materials for naphthodianthrones and phlorogluncinols is difficult, most reference standards are prepared through extraction and isolation from natural products and are especially likely to contain related impurities.

- If a calibration curve is used, the calibrant concentrations should encompass the sample concentrations. No sample concentrations should be outside of the linear range. 
- Individual matched calibrants should be used for quantitation whenever possible. For example, a rutin calibrant should not be used for the quantitation of hyperforin.

- Laboratories reporting results flagged as outliers should check for errors in calculations or reporting units. Confirm that all dilution factors have been properly tabulated. 
Table 6-1. Data summary table for phenolics in St. John's Wort.

\section{National Institute of Standards \& Technology}

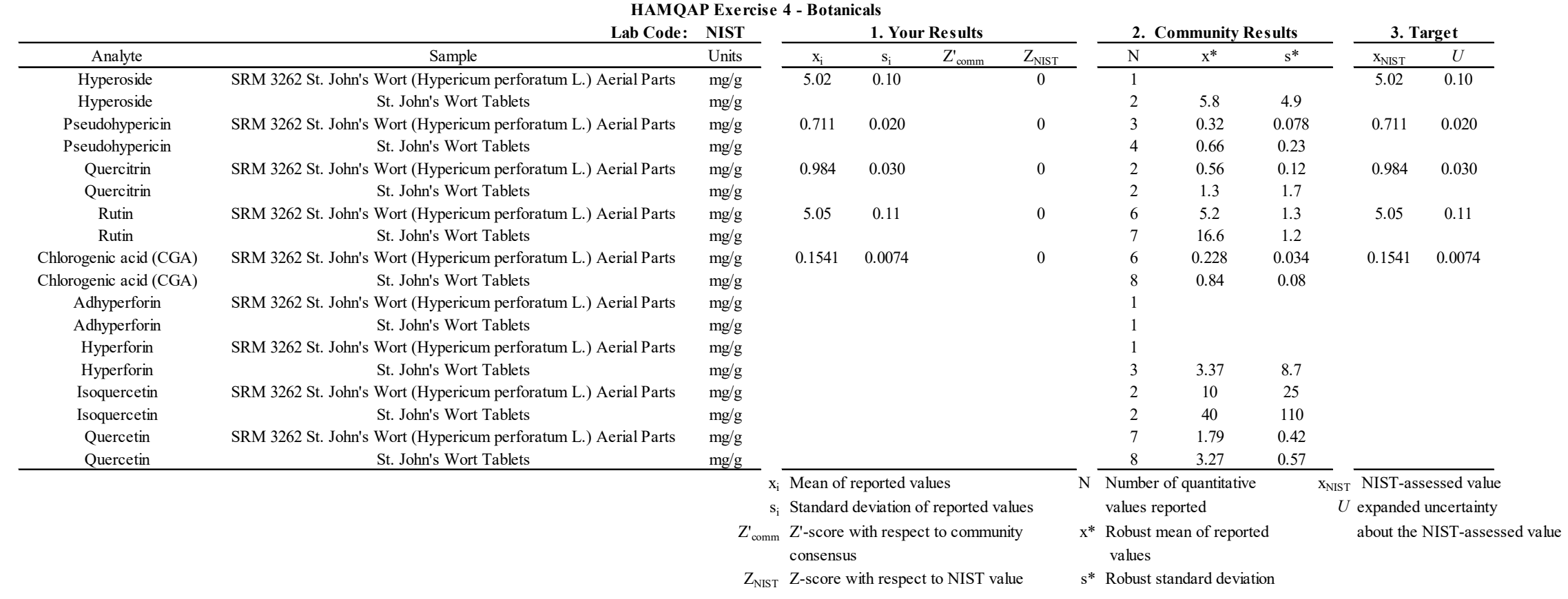


Table 6-2. Data summary table for hyperoside in St. John's Wort.

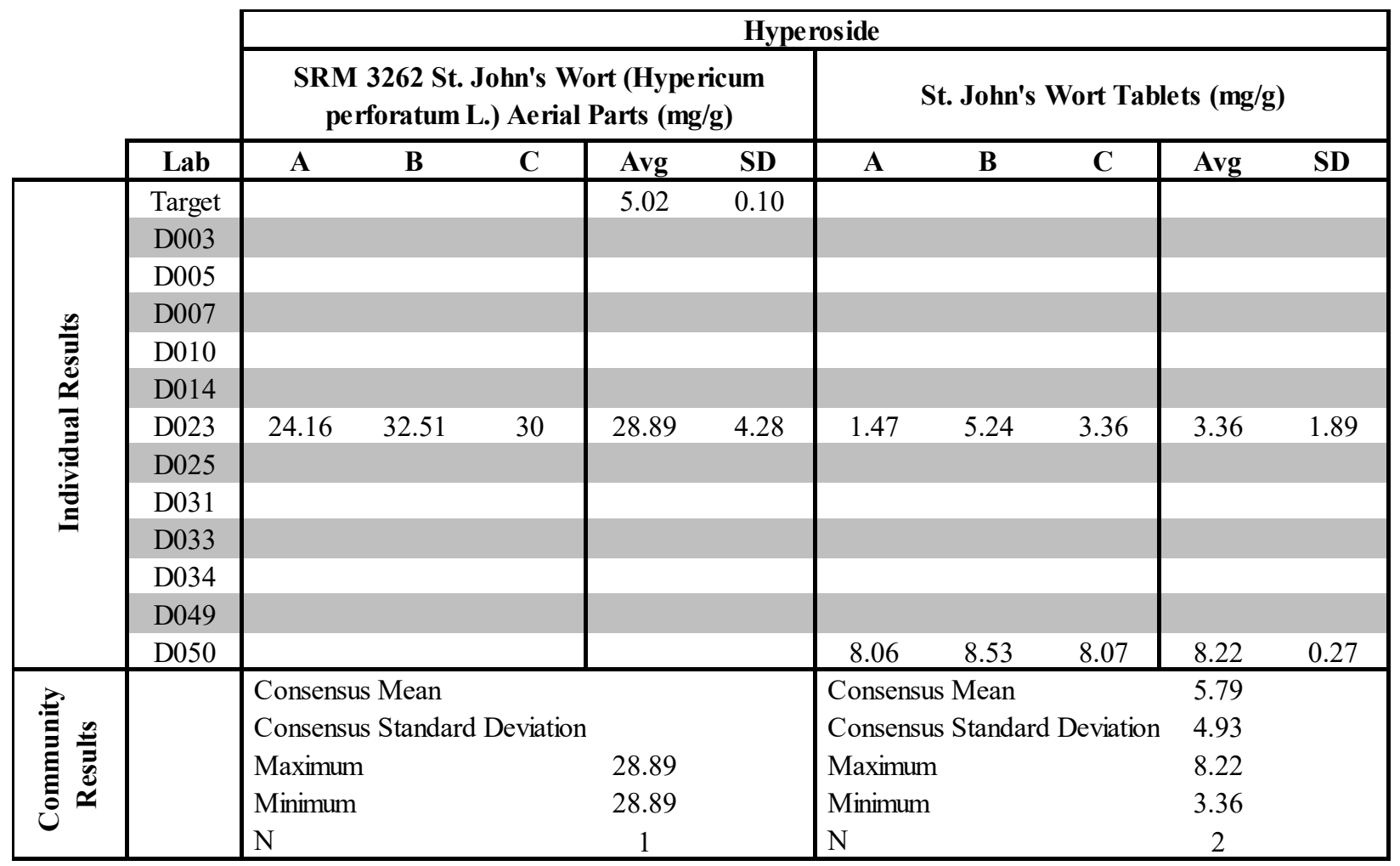




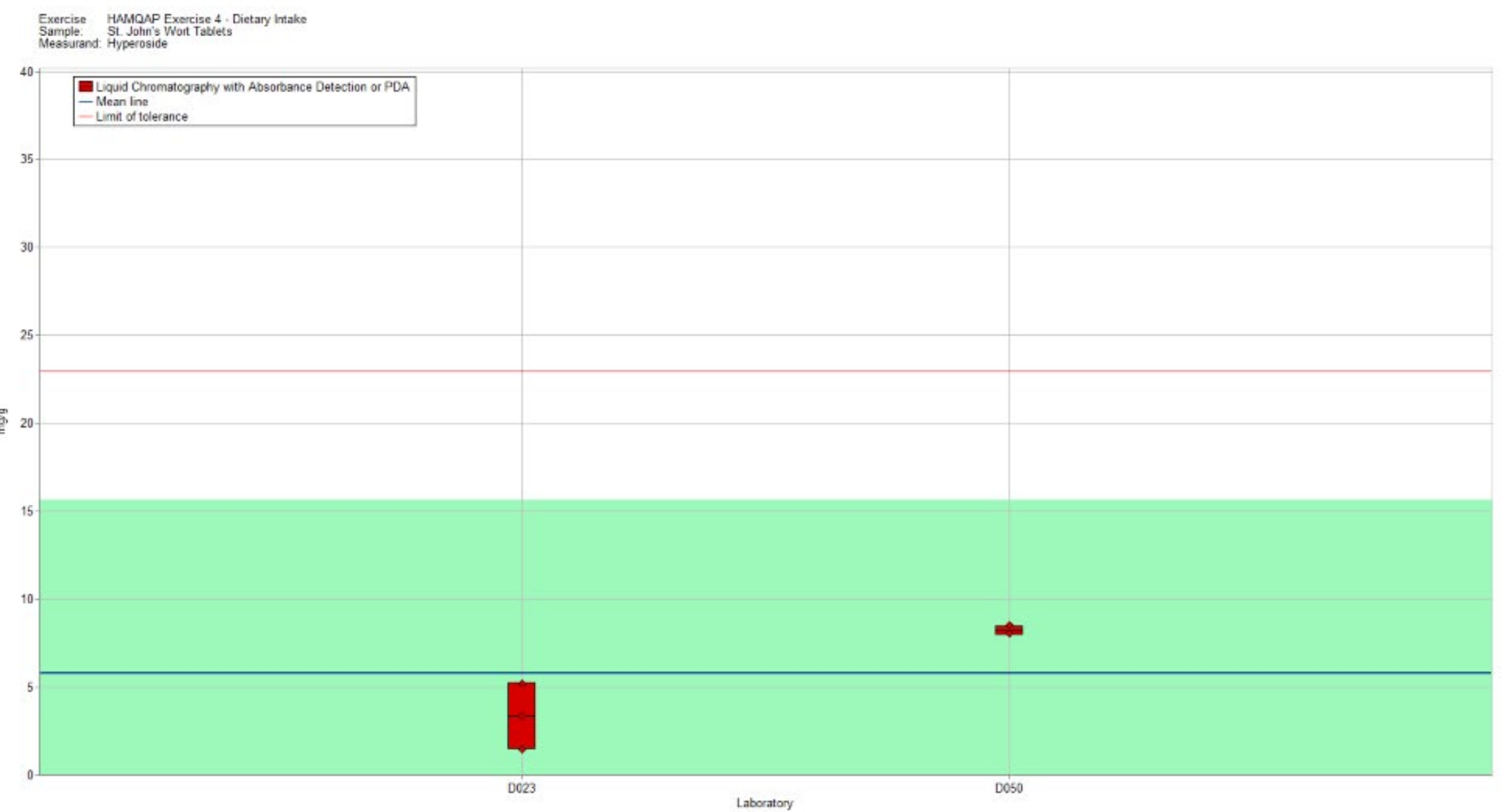

Figure 6-1. Hyperoside in St. John's Wort Tablets (data summary view - analytical method). In this view, individual laboratory data are plotted (diamonds) with the individual laboratory standard deviation (rectangle). The solid blue line represents the consensus mean, and the green shaded region represents the $95 \%$ confidence interval for the consensus mean. The solid red line represents the upper consensus range of tolerance, calculated as the values above the consensus mean that result in an acceptable $Z_{\text {comm }}^{\prime}$ score, $\left|Z_{\text {comm }}^{\prime}\right| \leq 2$, with the lower limit set at zero. A NIST value has not been determined in this material. 
Table 6-3. Data summary table for pseudohypericin in St. John's Wort. Data points highlighted in red have been flagged as potential outliers (e.g., Grubb and/or Cochran) by the NIST software package.

\begin{tabular}{|c|c|c|c|c|c|c|c|c|c|c|c|}
\hline & \multirow[b]{3}{*}{ Lab } & \multicolumn{10}{|c|}{ Pseudohypericin } \\
\hline & & $\begin{array}{r}\text { SRM } \\
\text { pe }\end{array}$ & $\begin{array}{l}262 \mathrm{St} \\
\text { oratum }\end{array}$ & $\begin{array}{l}\text { ohn's Wo } \\
\text { ) Aerial }\end{array}$ & $\begin{array}{l}\text { rt (Hyp } \\
\text { Parts (m }\end{array}$ & $\begin{array}{l}\text { icum } \\
\text { g) }\end{array}$ & & John's & Vort Tal & lets $(\mathrm{mg} / \mathrm{g}$ & \\
\hline & & $\mathbf{A}$ & B & $\mathrm{C}$ & Avg & SD & $\mathbf{A}$ & $\mathbf{B}$ & $\mathrm{C}$ & Avg & SD \\
\hline \multirow{13}{*}{ 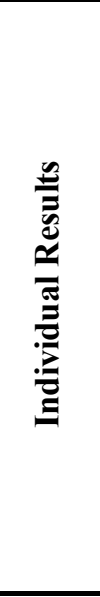 } & Target & & & & 0.711 & 0.020 & & & & & \\
\hline & D003 & & & & & & & & & & \\
\hline & D005 & & & & & & & & & & \\
\hline & D007 & & & & & & & & & & \\
\hline & D010 & & & & & & & & & & \\
\hline & D014 & 0.399 & 0.252 & 0.285 & 0.312 & 0.077 & 0.246 & 0.627 & 0.58 & 0.48433 & 0.21 \\
\hline & D023 & 11.73 & 17.68 & 16.16 & 15.190 & 3.091 & 0.75 & 2.4 & 1.58 & 1.57667 & 0.83 \\
\hline & D025 & & & & & & & & & & \\
\hline & D031 & & & & & & & & & & \\
\hline & D033 & 0.339 & 0.323 & 0.324 & 0.329 & 0.009 & 0.746 & 0.784 & 0.741 & 0.75700 & 0.02 \\
\hline & D034 & & & & & & & & & & \\
\hline & D049 & & & & & & & & & & \\
\hline & $\mathrm{D} 050$ & & & & & & 0.059 & 0.054 & 0.054 & 0.05567 & 0.00 \\
\hline \multirow{5}{*}{ 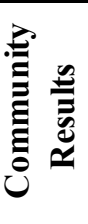 } & & \multicolumn{3}{|c|}{ Consensus Mean } & 0.320 & & \multicolumn{3}{|c|}{ Consensus Mean } & 0.66 & \\
\hline & & \multicolumn{3}{|c|}{ Consensus Standard Deviatio: } & 0.078 & & \multicolumn{4}{|c|}{ Consensus Standard Deviatio $\quad 0.23$} & \\
\hline & & \multirow{2}{*}{\multicolumn{3}{|c|}{ Minimum }} & 15.190 & & \multicolumn{3}{|c|}{ Maximum } & 1.58 & \\
\hline & & & & & 0.312 & & \multirow{2}{*}{\multicolumn{2}{|c|}{ Minimum }} & & 0.06 & \\
\hline & & \multicolumn{3}{|c|}{$\mathrm{N}$} & 3 & & & & & 4 & \\
\hline
\end{tabular}


Exercise HAMQAP Exercise 4 - Dietary Intake
Sample:
MRM $2362 \mathrm{St}$ John's Wort (Hypericum perforatum L.) Aerial Parts
Measurand. Pseudohypericin

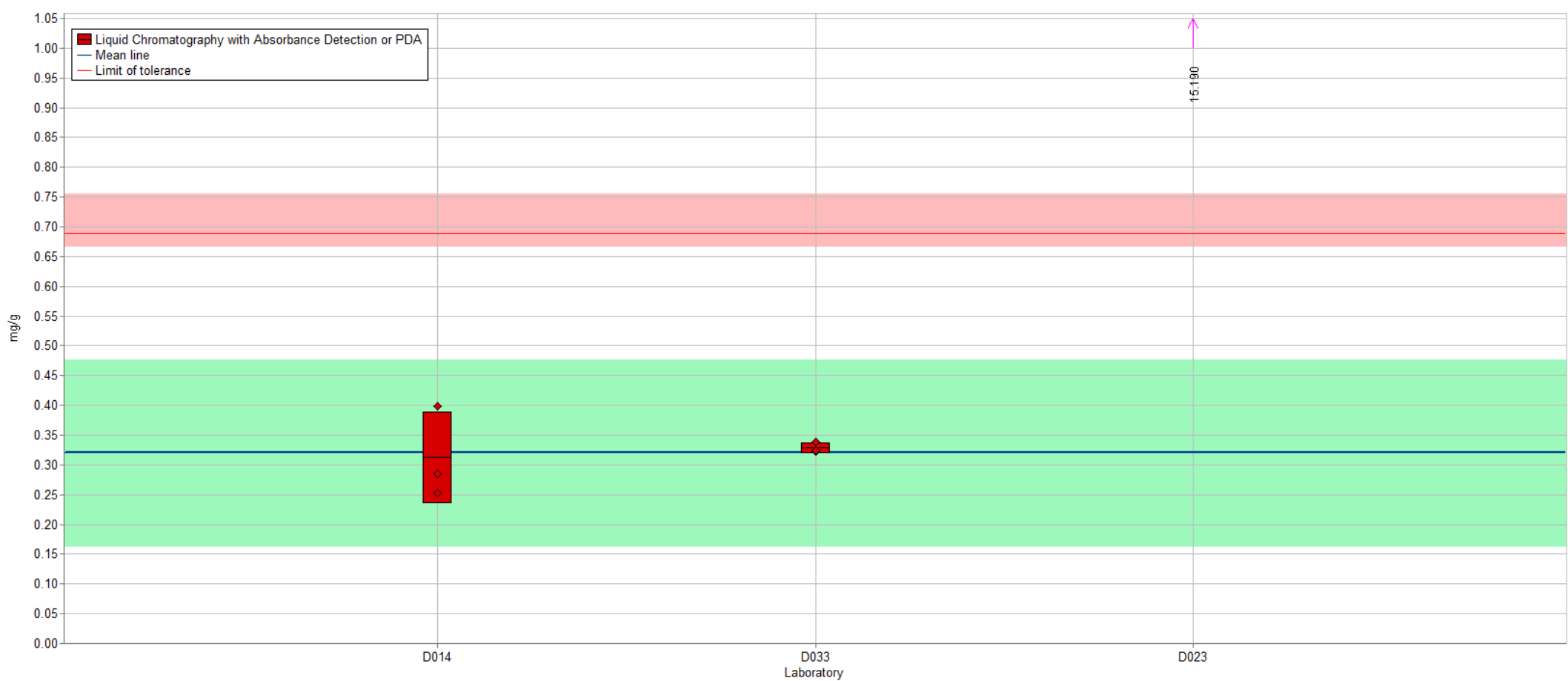

Figure 6-2. Pseudohypericin in SRM 3262 St. John's Wort (Hypericum perforatum L.) Aerial Parts (data summary view - analytical method). In this view, individual laboratory data are plotted (diamonds) with the individual laboratory standard deviation (rectangle). The solid blue line represents the consensus mean, and the green shaded region represents the $95 \%$ confidence interval for the consensus mean. The solid red line represents the upper consensus range of tolerance, calculated as the values above the consensus mean that result in an acceptable $Z_{\text {comm }}^{\prime}$ score, $\left|Z_{\text {comm }}^{\prime}\right| \leq 2$, with the lower limit set at zero. The red shaded region represents the NIST range of tolerance, which encompasses the target value bounded by its uncertainty ( $U_{\text {NIST }}$ ) and represents the range that results in an acceptable $Z_{\text {NIST }}$ score, $\left|Z_{\text {NIST }}\right| \leq 2$. 


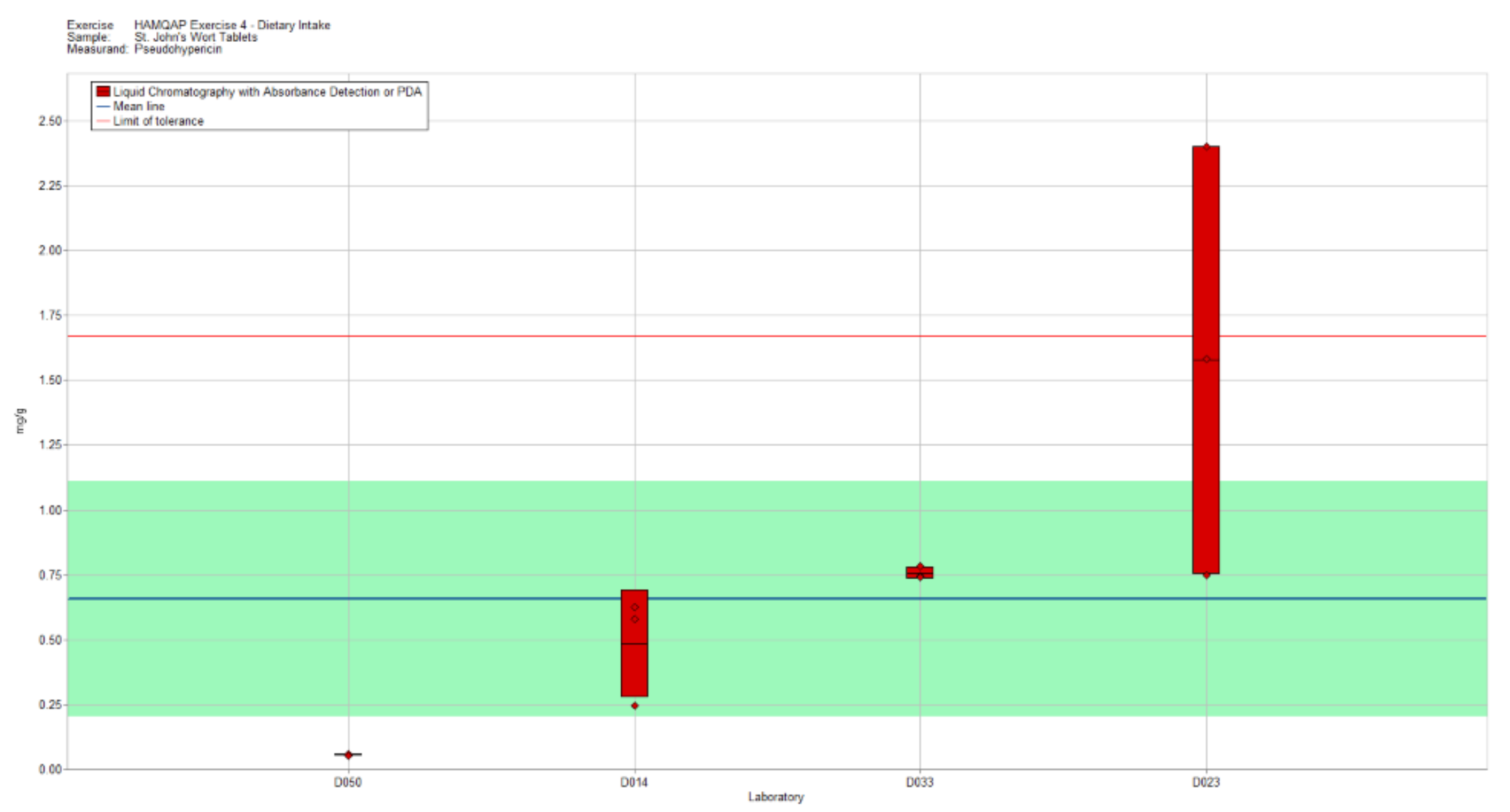

Figure 6-3. Pseudohypericin in St. John's Wort Tablets (data summary view - analytical method). In this view, individual laboratory data are plotted (diamonds) with the individual laboratory standard deviation (rectangle). The solid blue line represents the consensus mean, and the green shaded region represents the $95 \%$ confidence interval for the consensus mean. The solid red line represents the upper consensus range of tolerance, calculated as the values above the consensus mean that result in an acceptable $Z_{\text {comm }}^{\prime}$ score, $\left|Z_{\text {comm }}^{\prime}\right| \leq 2$, with the lower limit set at zero. A NIST value has not been determined in this material. 
Table 6-4. Data summary table for quercitrin in St. John's Wort.

\begin{tabular}{|c|c|c|c|c|c|c|c|c|c|c|c|}
\hline & \multirow[b]{3}{*}{ Lab } & \multicolumn{10}{|c|}{ Quercitrin } \\
\hline & & \multicolumn{5}{|c|}{$\begin{array}{l}\text { SRM } 3262 \text { St. John's Wort (Hypericum } \\
\text { perforatum L.) Aerial Parts (mg/g) }\end{array}$} & \multicolumn{5}{|c|}{ St. John's Wort Tablets (mg/g) } \\
\hline & & $\mathbf{A}$ & B & $\mathbf{C}$ & Avg & SD & $\mathbf{A}$ & B & $\mathbf{C}$ & Avg & SD \\
\hline \multirow{12}{*}{ 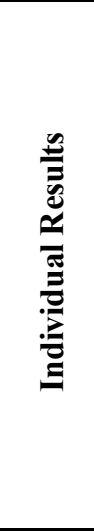 } & Target & & & & 0.984 & 0.030 & & & & & \\
\hline & D003 & & & & & & & & & & \\
\hline & D005 & & & & & & & & & & \\
\hline & D007 & & & & & & & & & & \\
\hline & D010 & & & & & & & & & & \\
\hline & D014 & 0.638 & 0.595 & 0.604 & 0.612 & 0.023 & 1.92 & 1.76 & 1.87 & 1.850 & 0.082 \\
\hline & D023 & 0.51 & 0.51 & 0.53 & 0.517 & 0.012 & 0.69 & 0.7 & 0.7 & 0.697 & 0.006 \\
\hline & D025 & & & & & & & & & & \\
\hline & D031 & & & & & & & & & & \\
\hline & D033 & $<1.00$ & $<1.00$ & $<1.00$ & & & $<1.00$ & $<1.00$ & $<1.00$ & & \\
\hline & D034 & & & & & & & & & & \\
\hline & D049 & & & & & & & & & & \\
\hline \multirow{5}{*}{ 泀 } & & \multirow{5}{*}{\multicolumn{3}{|c|}{$\begin{array}{l}\text { Consensus Mean } \\
\text { Consensus Standard Deviation } \\
\text { Maximum } \\
\text { Minimum } \\
\mathrm{N}\end{array}$}} & 0.565 & & \multirow{5}{*}{\multicolumn{3}{|c|}{$\begin{array}{l}\text { Consensus Mean } \\
\text { Consensus Standard Deviation } \\
\text { Maximum } \\
\text { Minimum } \\
\mathrm{N}\end{array}$}} & 1.273 & \\
\hline & & & & & 0.124 & & & & & 1.676 & \\
\hline & & & & & 0.612 & & & & & 1.850 & \\
\hline & & & & & 0.517 & & & & & 0.697 & \\
\hline & & & & & 2 & & & & & 2 & \\
\hline
\end{tabular}




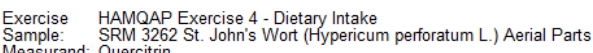

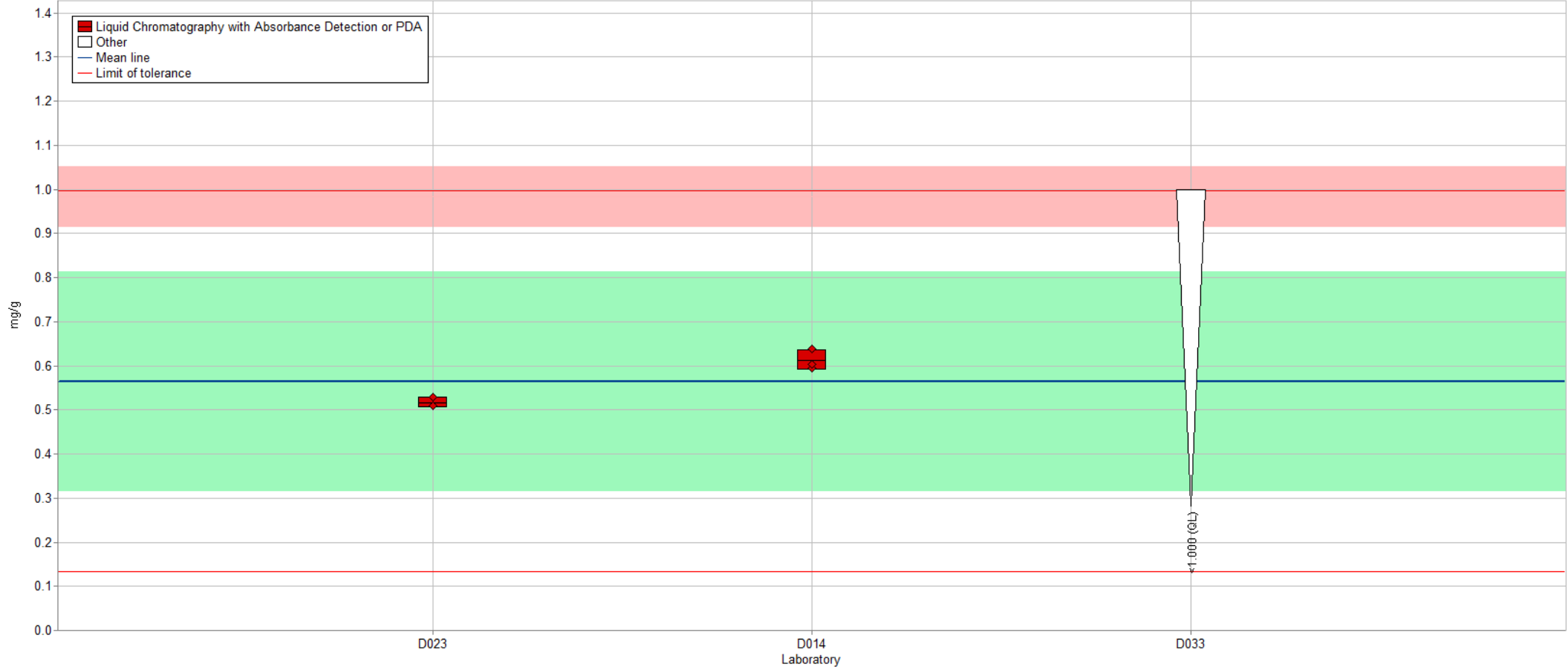

Figure 6-4. Quercitrin in SRM 3262 St. John's Wort (Hypericum perforatum L.) Aerial Parts (data summary view - analytical method). In this view, individual laboratory data are plotted (diamonds) with the individual laboratory standard deviation (rectangle). The solid blue line represents the consensus mean, and the green shaded region represents the $95 \%$ confidence interval for the consensus mean. The solid red line represents the upper consensus range of tolerance, calculated as the values above and below the consensus mean that result in an acceptable $Z_{\text {comm }}^{\prime}$ score, $\left|Z_{\text {comm }}^{\prime}\right| \leq 2$. The red shaded region represents the NIST range of tolerance, which encompasses the target value bounded by its uncertainty ( $U_{\text {NIST }}$ ) and represents the range that results in an acceptable $Z_{\text {NIST }}$ score, $\left|Z_{\text {NIST }}\right| \leq 2$. 


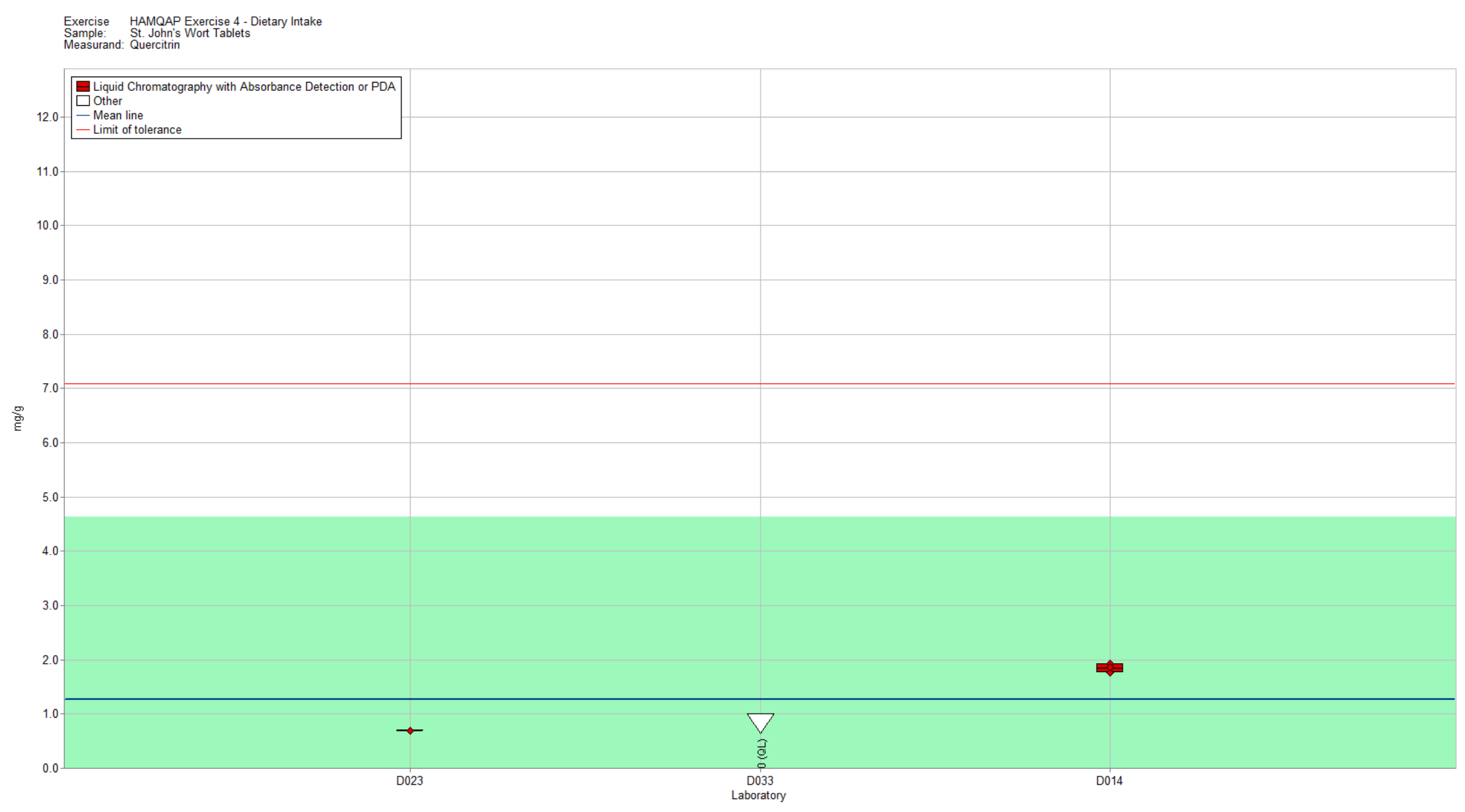

Figure 6-5. Quercitrin in St. John's Wort Tablets (data summary view - analytical method). In this view, individual laboratory data are plotted (diamonds) with the individual laboratory standard deviation (rectangle). The solid blue line represents the consensus mean, and the green shaded region represents the $95 \%$ confidence interval for the consensus mean. The solid red line represents the upper consensus range of tolerance, calculated as the values above the consensus mean that result in an acceptable $Z_{\text {comm }}^{\prime}$ score, $\left|Z_{\text {comm }}^{\prime}\right| \leq 2$, with the lower limit set at zero. A NIST value has not been determined in this material. 
Table 6-5. Data summary table for rutin in St. John's Wort. Data points highlighted in red have been flagged as potential outliers (e.g., Grubb and/or Cochran) by the NIST software package.

\begin{tabular}{|c|c|c|c|c|c|c|c|c|c|c|c|}
\hline & \multirow[b]{3}{*}{ Lab } & \multicolumn{10}{|c|}{ Rutin } \\
\hline & & \multicolumn{5}{|c|}{$\begin{array}{l}\text { SRM } 3262 \text { St. John's Wort (Hypericum } \\
\text { perforatum L.) Aerial Parts (mg/g) }\end{array}$} & \multicolumn{5}{|c|}{ St. John's Wort Tablets (mg/g) } \\
\hline & & $\mathbf{A}$ & B & $\mathbf{C}$ & Avg & SD & $\mathbf{A}$ & $\mathbf{B}$ & $\mathbf{C}$ & Avg & SD \\
\hline \multirow{17}{*}{ 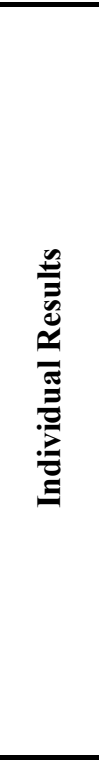 } & Target & & & & 5.05 & 0.11 & & & & & \\
\hline & D003 & & & & & & & & & & \\
\hline & D004 & 7.75 & 7.73 & 7.82 & 7.77 & 0.05 & 23.25 & 23.41 & 22.95 & 23.20 & 0.23 \\
\hline & D005 & & & & & & & & & & \\
\hline & D007 & & & & & & & & & & \\
\hline & D009 & 12.88 & 12.71 & 12.83 & 12.81 & 0.09 & 32.35 & 32.27 & 31.63 & 32.08 & 0.39 \\
\hline & D010 & & & & & & & & & & \\
\hline & D014 & 2.53 & 2.27 & 1.97 & 2.26 & 0.28 & 16.1 & 16.4 & 15.4 & 15.97 & 0.51 \\
\hline & D017 & 3.2 & 3.2 & 3.1 & 3.17 & 0.06 & 15.4 & 15.1 & 15.7 & 15.40 & 0.30 \\
\hline & D023 & 3.17 & 3.37 & 3.34 & 3.29 & 0.11 & 16.69 & 16.17 & 14.93 & 15.93 & 0.90 \\
\hline & D025 & & & & & & & & & & \\
\hline & D031 & & & & & & & & & & \\
\hline & D033 & 4.52 & 4.76 & 4.69 & 4.66 & 0.12 & 17.2 & 17.5 & 16.5 & 17.07 & 0.51 \\
\hline & D034 & & & & & & & & & & \\
\hline & D046 & & & & & & & & & & \\
\hline & D049 & & & & & & & & & & \\
\hline & D050 & & & & & & 13.44 & 14.47 & 13.93 & 13.95 & 0.52 \\
\hline & & Consen & Mean & & 5.16 & & Consen & Mean & & 16.60 & \\
\hline$\cong$ & & Consen & Standa & Deviation & 1.26 & & Consen & Standar & Deviation & 1.17 & \\
\hline$\overline{\bar{z}}$ & & Maximu & & & 12.81 & & Maximu & & & 32.08 & \\
\hline$\ddot{0}$ & & Minimu & & & 2.26 & & Minimur & & & 13.95 & \\
\hline & & $\mathrm{N}$ & & & 6 & & $\mathrm{~N}$ & & & 7 & \\
\hline
\end{tabular}


$\begin{array}{ll}\text { Exercise } & \text { HAMQAP Exercise } 4 \text { - Dietary Intake } \\ \text { Sample: } & \text { SRM } 3262 \text { St. John's Wort (Hypericum perforatum L.) Aerial Parts } \\ \text { Measurand. } & \text { Rutin }\end{array}$

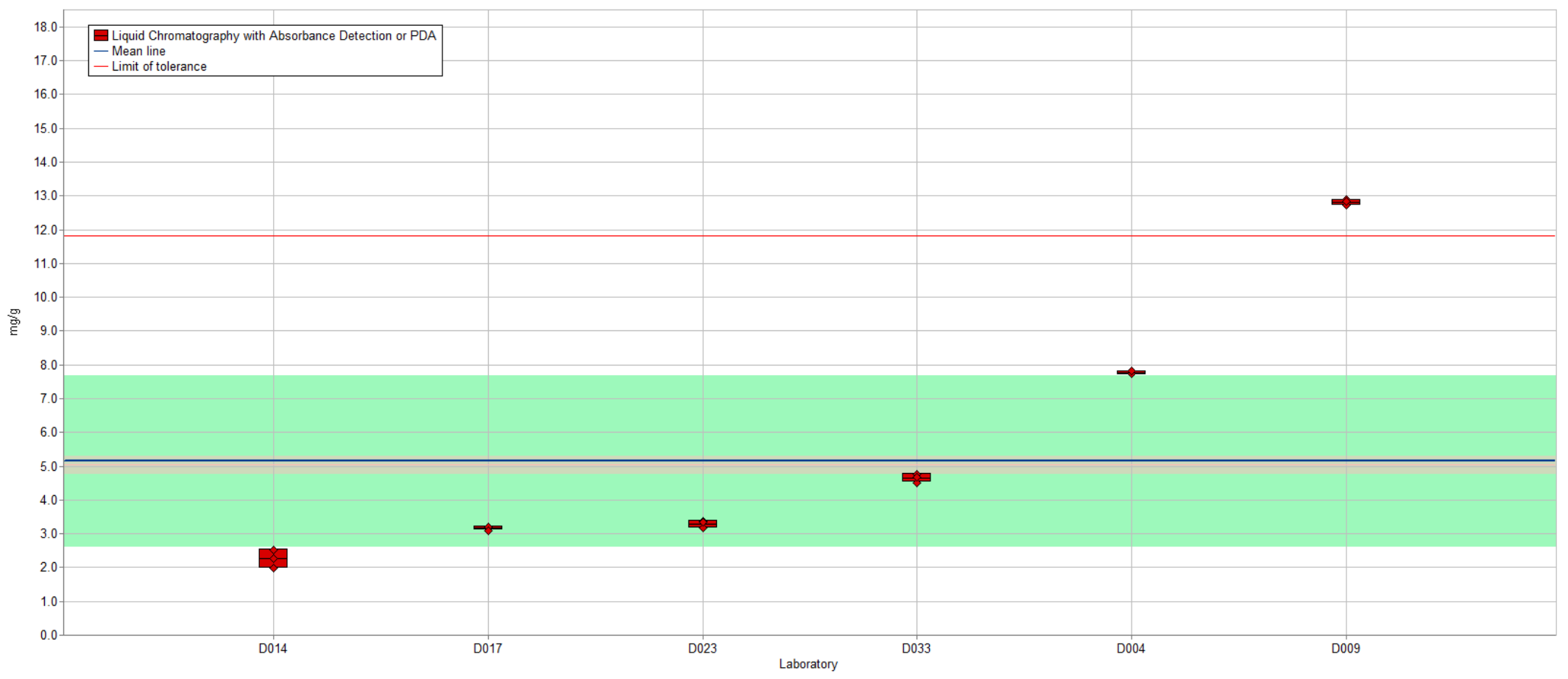

Figure 6-6. Rutin in SRM 3262 St. John's Wort (Hypericum perforatum L.) Aerial Parts (data summary view - analytical method). In this view, individual laboratory data are plotted (diamonds) with the individual laboratory standard deviation (rectangle). The solid blue line represents the consensus mean, and the green shaded region represents the $95 \%$ confidence interval for the consensus mean. The solid red line represents the upper consensus range of tolerance, calculated as the values above the consensus mean that result in an acceptable $Z_{\text {comm }}^{\prime}$ score, $\left|Z_{\text {comm }}^{\prime}\right| \leq 2$, with the lower limit set at zero. The red shaded region represents the NIST range of tolerance, which encompasses the target value bounded by its uncertainty ( $U_{\text {NIST}}$ ) and represents the range that results in an acceptable $Z_{\text {NIST }}$ score, $\left|Z_{\mathrm{NIST}}\right| \leq 2$. 


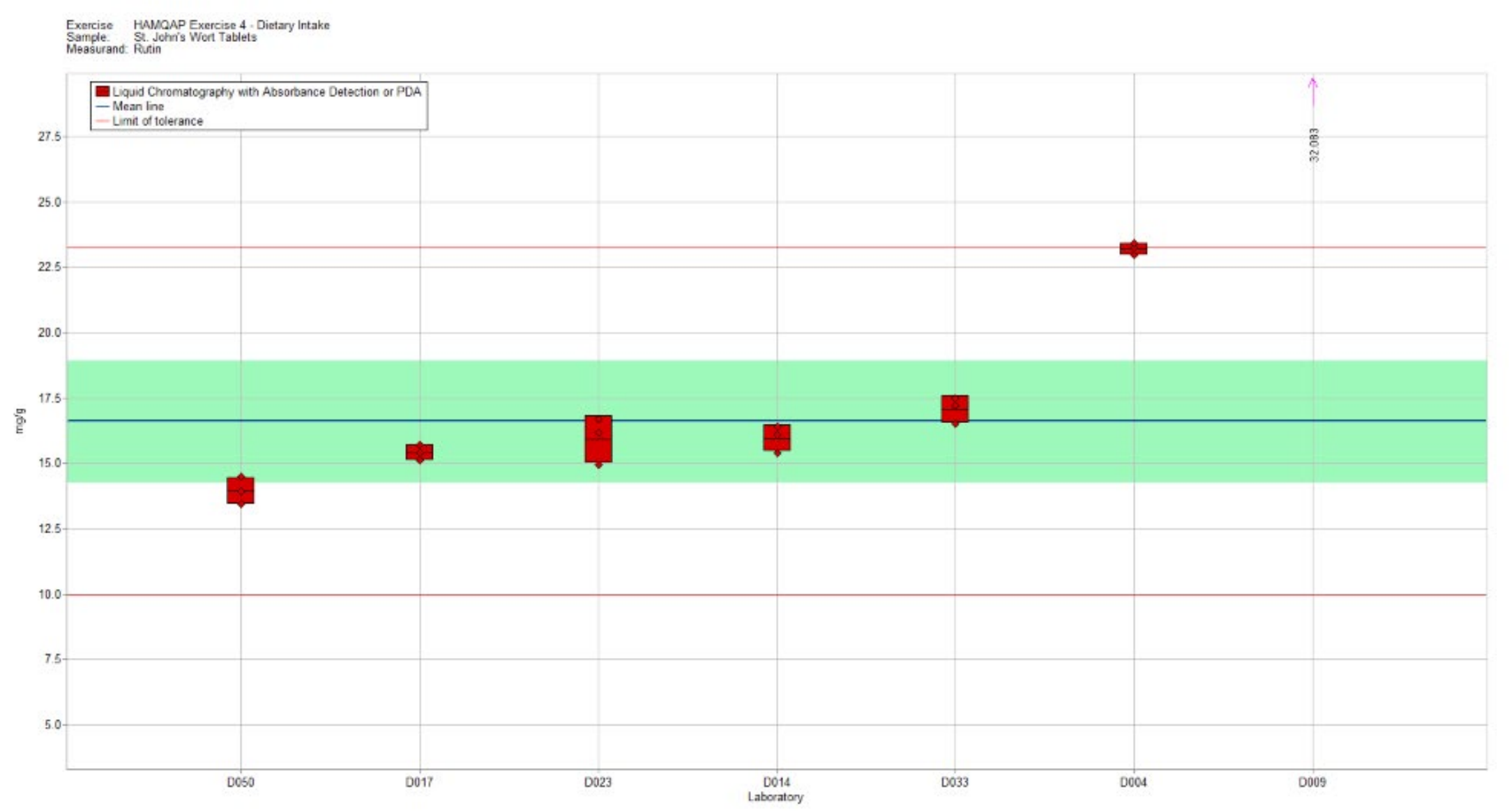

Figure 6-7. Rutin in St. John's Wort Tablets (data summary view - analytical method). In this view, individual laboratory data are plotted (diamonds) with the individual laboratory standard deviation (rectangle). The solid blue line represents the consensus mean, and the green shaded region represents the $95 \%$ confidence interval for the consensus mean. The solid red lines represent the consensus range of tolerance, calculated as the values above and below the consensus mean that result in an acceptable $Z_{\text {comm }}^{\prime}$ score, $\left|Z_{\text {comm }}^{\prime}\right| \leq 2$. A NIST value has not been determined in this material. 
Table 6-6. Data summary table for chlorogenic acid in St. John's Wort. Data points highlighted in red have been flagged as potential outliers (e.g., Grubb and/or Cochran) by the NIST software package.

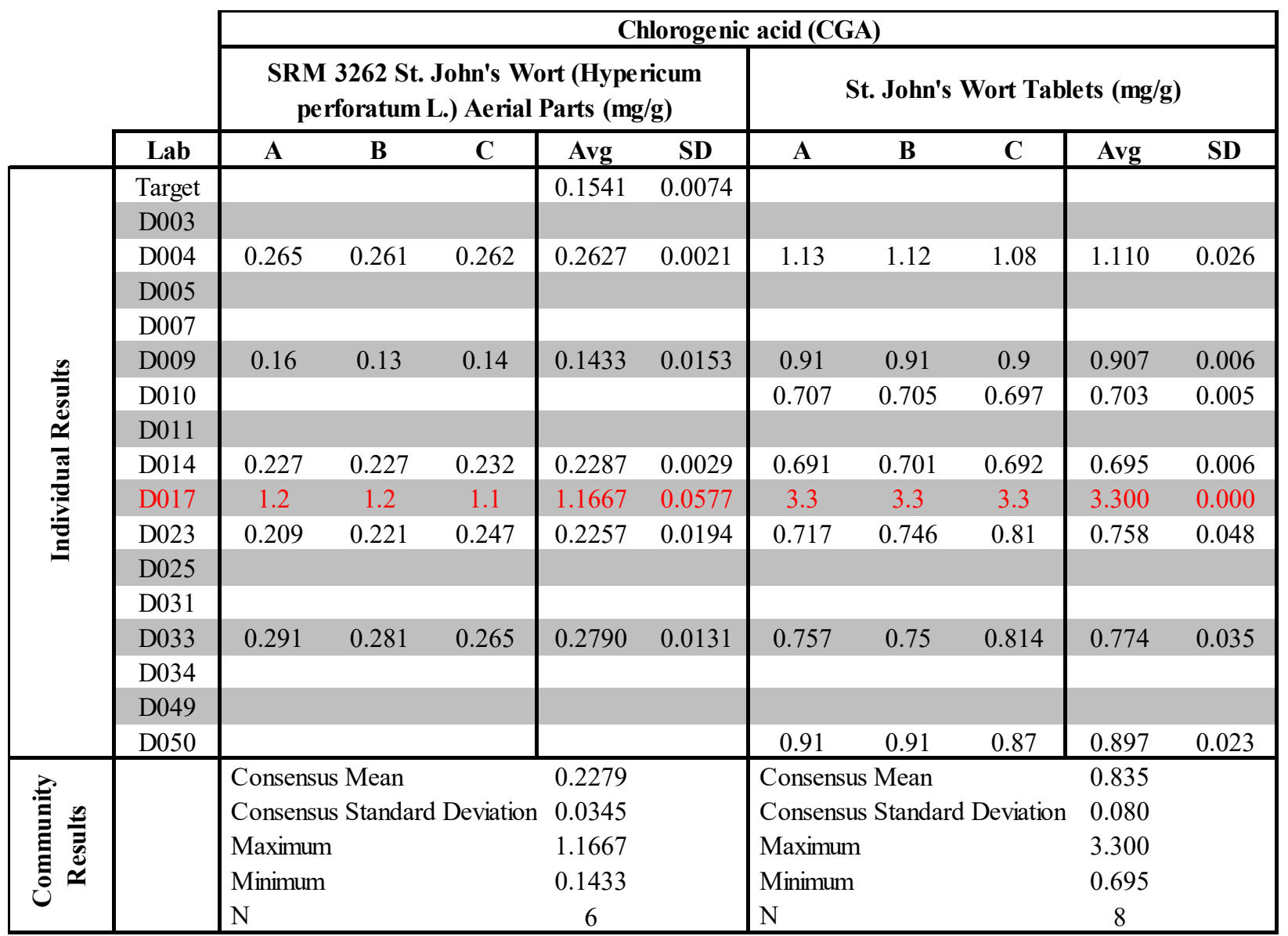




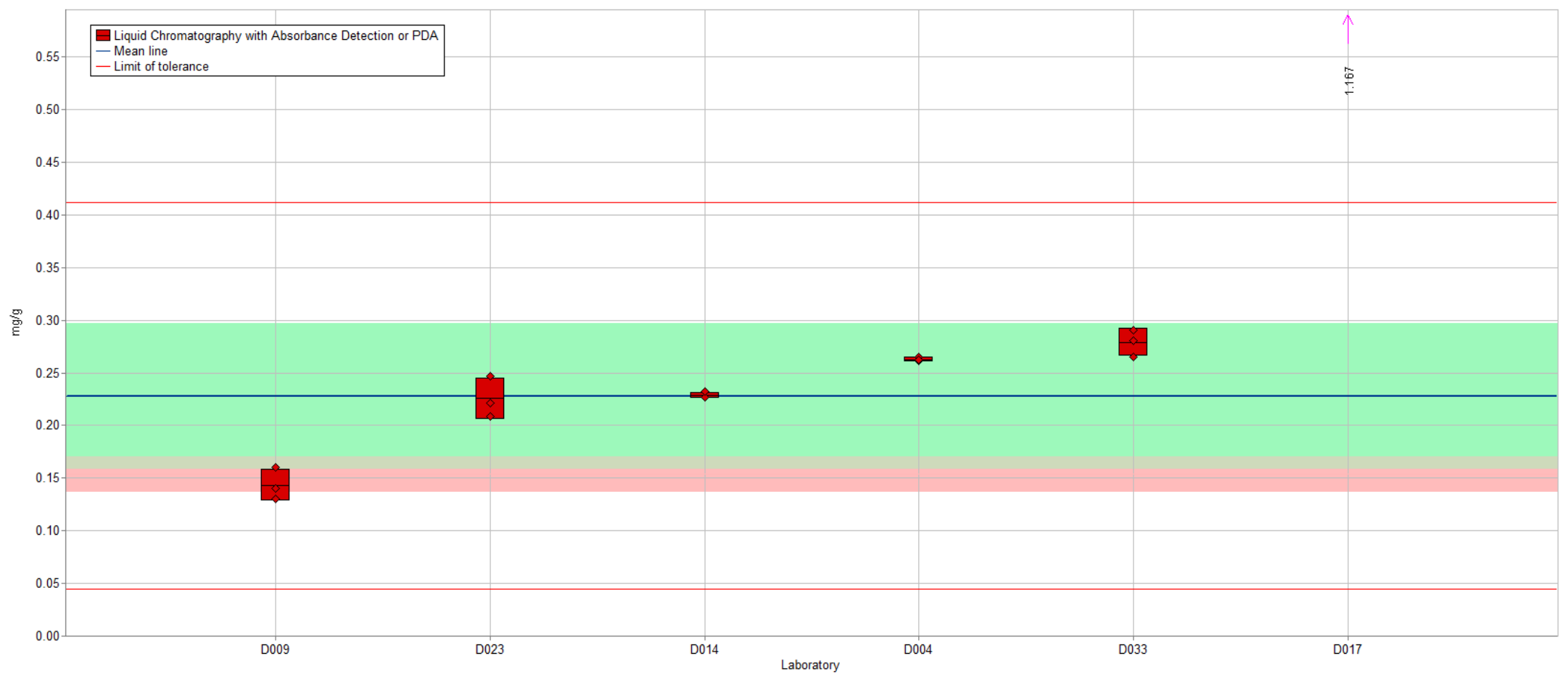

Figure 6-8. Chlorogenic acid in SRM 3262 St. John's Wort (Hypericum perforatum L.) Aerial Parts (data summary view - analytical method). In this view, individual laboratory data are plotted (diamonds) with the individual laboratory standard deviation (rectangle). The solid blue line represents the consensus mean, and the green shaded region represents the $95 \%$ confidence interval for the consensus mean. The solid red line represents the upper consensus range of tolerance, calculated as the values above and below the consensus mean that result in an acceptable $Z_{\text {comm }}^{\prime}$ score, $\left|Z_{\text {comm }}^{\prime}\right| \leq 2$. The red shaded region represents the NIST range of tolerance, which encompasses the target value bounded by its uncertainty $\left(U_{\mathrm{NIST}}\right)$ and represents the range that results in an acceptable $Z_{\mathrm{NIST}}$ score, $\left|Z_{\mathrm{NIST}}\right| \leq 2$. 


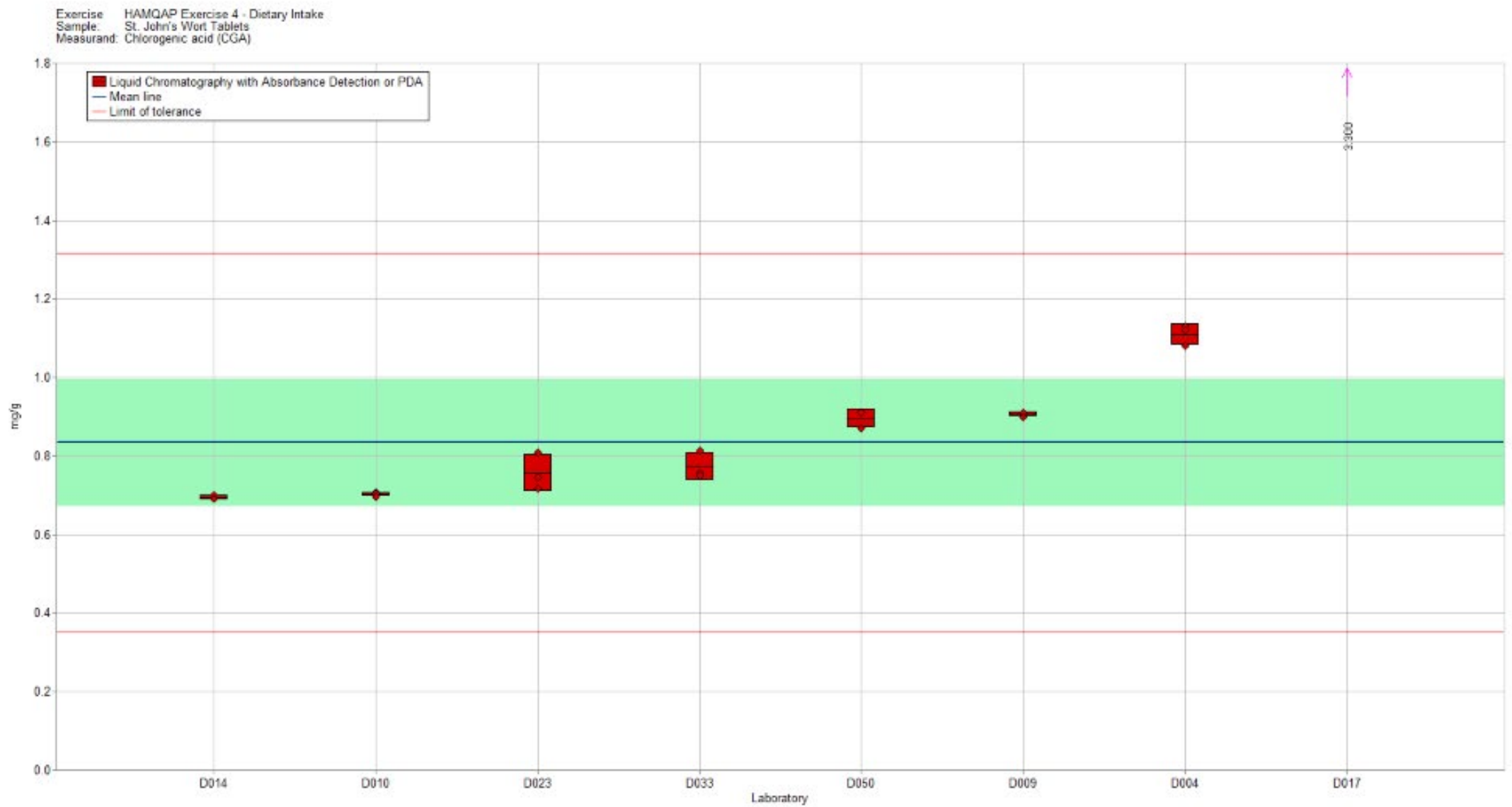

Figure 6-9. Chlorogenic acid in St. John's Wort Tablets (data summary view - analytical method). In this view, individual laboratory data are plotted (diamonds) with the individual laboratory standard deviation (rectangle). The solid blue line represents the consensus mean, and the green shaded region represents the $95 \%$ confidence interval for the consensus mean. The solid red lines represent the consensus range of tolerance, calculated as the values above and below the consensus mean that result in an acceptable $Z_{\text {comm }}^{\prime}$ score, $\left|Z_{\text {comm }}^{\prime}\right| \leq 2$. A NIST value has not been determined in this material. 
Table 6-7. Data summary table for adhyperforin in St. John's Wort.

\begin{tabular}{|c|c|c|c|c|c|c|c|c|c|c|c|}
\hline & \multirow[b]{3}{*}{ Lab } & \multicolumn{10}{|c|}{ Adhyperforin } \\
\hline & & \multicolumn{5}{|c|}{$\begin{array}{l}\text { SRM } 3262 \text { St. John's Wort (Hypericum } \\
\text { perforatum L.) Aerial Parts (mg/g) }\end{array}$} & \multicolumn{5}{|c|}{ St. John's Wort Tablets (mg/g) } \\
\hline & & $\mathbf{A}$ & $\mathbf{B}$ & $\mathbf{C}$ & Avg & SD & $\mathbf{A}$ & B & $\mathbf{C}$ & Avg & SD \\
\hline \multirow{11}{*}{ 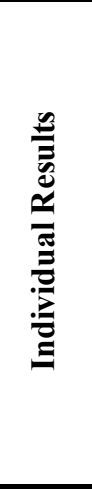 } & Target & & & & & & & & & & \\
\hline & D003 & & & & & & & & & & \\
\hline & D005 & & & & & & & & & & \\
\hline & D007 & & & & & & & & & & \\
\hline & D010 & & & & & & & & & & \\
\hline & D023 & 65.18 & 56.42 & 57.35 & 59.7 & 4.8 & 92.07 & 82.26 & 87.17 & 87.2 & 4.9 \\
\hline & D025 & & & & & & & & & & \\
\hline & D031 & & & & & & & & & & \\
\hline & D033 & & & & & & & & & & \\
\hline & D034 & & & & & & & & & & \\
\hline & D049 & & & & & & & & & & \\
\hline 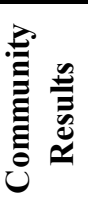 & & $\begin{array}{l}\text { Consen } \\
\text { Consen } \\
\text { Maximu } \\
\text { Minimur } \\
\mathrm{N}\end{array}$ & $\begin{array}{l}\text { Mean } \\
\text { Standa }\end{array}$ & Deviatio & $\begin{array}{c}59.7 \\
59.7 \\
1\end{array}$ & & $\begin{array}{l}\text { Consen } \\
\text { Consen } \\
\text { Maxim } \\
\text { Minimu } \\
\mathrm{N}\end{array}$ & $\begin{array}{l}\text { Mean } \\
\text { Standa }\end{array}$ & Deviatio & $\begin{array}{c}87.2 \\
87.2 \\
1\end{array}$ & \\
\hline
\end{tabular}


Table 6-8. Data summary table for hyperforin in St. John's Wort. Data points highlighted in red have been flagged as potential outliers (e.g., Grubb and/or Cochran) by the NIST software package.

\begin{tabular}{|c|c|c|c|c|c|c|c|c|c|c|c|}
\hline & \multirow[b]{3}{*}{ Lab } & \multicolumn{10}{|c|}{ Hyperforin } \\
\hline & & & $\begin{array}{l}262 \mathrm{~S} \\
\text { oratu }\end{array}$ & $\begin{array}{l}\text { ohn's } \\
\text { ) Aeria }\end{array}$ & $\begin{array}{l}\text { t }(\mathrm{Hyp} \\
\text { arts (n }\end{array}$ & & & John's & Vort Tab & ts (mg/ & \\
\hline & & $\mathbf{A}$ & B & $\mathrm{C}$ & Avg & SD & $\mathbf{A}$ & B & $\mathrm{C}$ & Avg & SD \\
\hline \multirow{10}{*}{ 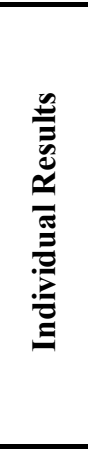 } & Target & & & & & & & & & & \\
\hline & D003 & & & & & & & & & & \\
\hline & D005 & & & & & & & & & & \\
\hline & D023 & 67 & 58.2 & 61.39 & 62.2 & 4.5 & 94.32 & 85.44 & 89.88 & 89.88 & 4.44 \\
\hline & D025 & & & & & & & & & & \\
\hline & D031 & & & & & & & & & & \\
\hline & D033 & & & & & & 6.57 & 6.8 & 6.78 & 6.72 & 0.13 \\
\hline & D042 & & & & & & & & & & \\
\hline & D049 & & & & & & & & & & \\
\hline & D050 & & & & & & 0.029 & 0.027 & 0.028 & 0.03 & 0.00 \\
\hline \multirow{5}{*}{ 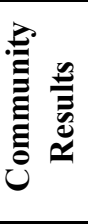 } & & \multirow{5}{*}{\multicolumn{3}{|c|}{$\begin{array}{l}\text { Consensus Mean } \\
\text { Consensus Standard Deviation } \\
\text { Maximum } \\
\text { Minimum } \\
\mathrm{N} \\
\end{array}$}} & & & \multirow{5}{*}{\multicolumn{3}{|c|}{$\begin{array}{l}\text { Consensus Mean } \\
\text { Consensus Standard Deviation } \\
\text { Maximum } \\
\text { Minimum } \\
\mathrm{N} \\
\end{array}$}} & 3.37 & \\
\hline & & & & & & & & & & 8.67 & \\
\hline & & & & & 62.2 & & & & & 89.88 & \\
\hline & & & & & 62.2 & & & & & 0.03 & \\
\hline & & & & & 1 & & & & & 3 & \\
\hline
\end{tabular}




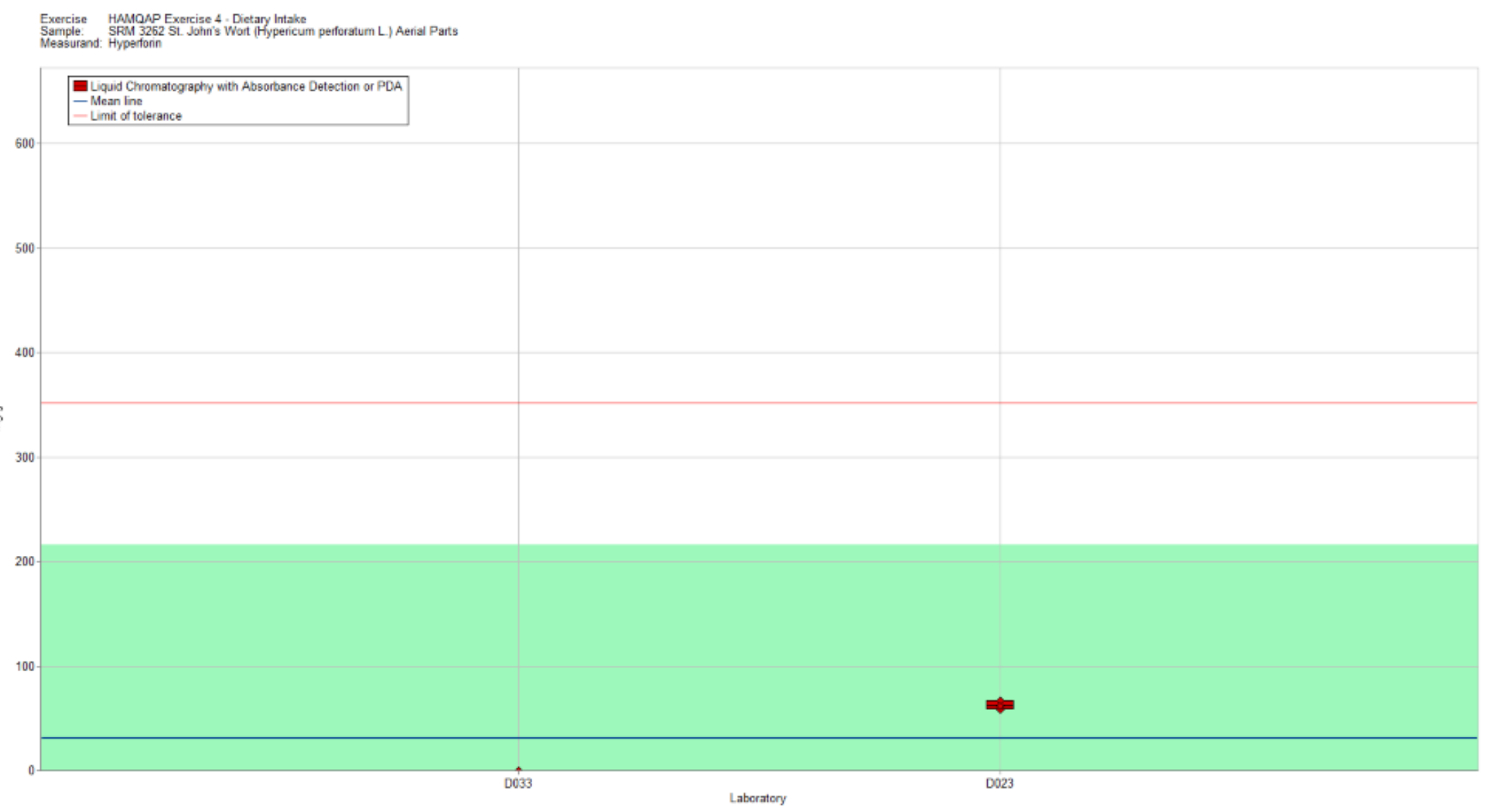

Figure 6-10. Hyperforin in in SRM 3262 St. John's Wort (Hypericum perforatum L.) Aerial Parts (data summary view - analytical method). In this view, individual laboratory data are plotted (diamonds) with the individual laboratory standard deviation (rectangle). The solid blue line represents the consensus mean, and the green shaded region represents the $95 \%$ confidence interval for the consensus mean. The solid red line represents the upper consensus range of tolerance, calculated as the values above the consensus mean that result in an acceptable $Z_{\text {comm }}^{\prime}$ score, $\left|Z_{\text {comm }}^{\prime}\right| \leq 2$, with the lower limit set at zero. A NIST value has not been determined in this material. 


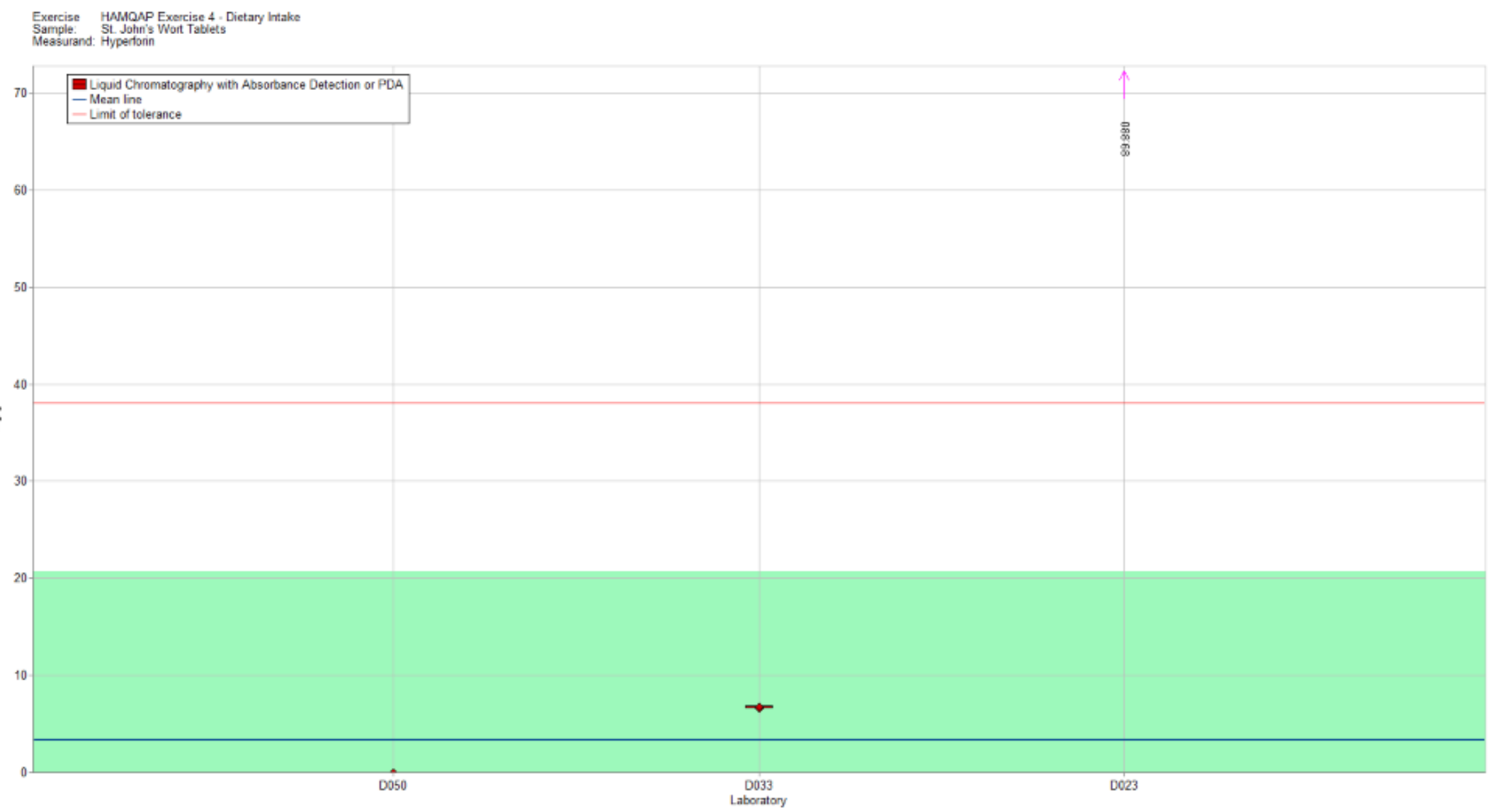

Figure 6-11. Hyperforin in St. John's Wort Tablets (data summary view - analytical method). In this view, individual laboratory data are plotted (diamonds) with the individual laboratory standard deviation (rectangle). The solid blue line represents the consensus mean, and the green shaded region represents the $95 \%$ confidence interval for the consensus mean. The solid red line represents the upper consensus range of tolerance, calculated as the values above the consensus mean that result in an acceptable $Z_{\text {comm }}^{\prime}$ score, $\left|Z_{\text {comm }}^{\prime}\right| \leq 2$, with the lower limit set at zero. A NIST value has not been determined in this material. 
Table 6-9. Data summary table for isoquercetin in St. John's Wort.

\begin{tabular}{|c|c|c|c|c|c|c|c|c|c|c|c|}
\hline & \multirow[b]{3}{*}{ Lab } & \multicolumn{10}{|c|}{ Isoquercetin } \\
\hline & & \multicolumn{5}{|c|}{$\begin{array}{l}\text { SRM } 3262 \text { St. John's Wort (Hypericum } \\
\text { perforatum L.) Aerial Parts (mg/g) }\end{array}$} & \multicolumn{5}{|c|}{ St. John's Wort Tablets (mg/g) } \\
\hline & & $\bar{A}$ & $\mathbf{B}$ & $\mathbf{C}$ & Avg & SD & $\bar{A}$ & $\mathbf{B}$ & $\mathrm{C}$ & Avg & SD \\
\hline \multirow{13}{*}{ 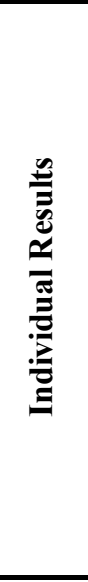 } & Target & & & & & & & & & & \\
\hline & D003 & & & & & & & & & & \\
\hline & D005 & & & & & & & & & & \\
\hline & D007 & & & & & & & & & & \\
\hline & D009 & & & & & & & & & & \\
\hline & D010 & & & & & & & & & & \\
\hline & D014 & 1.23 & 1.1 & 1.25 & 1.19 & 0.08 & 5.68 & 5.8 & 5.53 & 5.67 & 0.14 \\
\hline & D023 & 17.44 & 17.4 & 19.11 & 18.0 & 1.0 & 76.46 & 75.31 & 76.62 & 76.1 & 0.7 \\
\hline & D025 & & & & & & & & & & \\
\hline & D031 & & & & & & & & & & \\
\hline & D033 & & & & & & & & & & \\
\hline & D034 & & & & & & & & & & \\
\hline & D049 & & & & & & & & & & \\
\hline \multirow{5}{*}{ 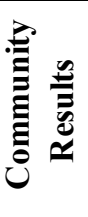 } & & \multirow{5}{*}{\multicolumn{3}{|c|}{$\begin{array}{l}\text { Consensus Mean } \\
\text { Consensus Standard Deviation } \\
\text { Maximum } \\
\text { Minimum } \\
\text { N }\end{array}$}} & 9.6 & & \multirow{5}{*}{\multicolumn{3}{|c|}{$\begin{array}{l}\text { Consensus Mean } \\
\text { Consensus Standa } \\
\text { Maximum } \\
\text { Minimum } \\
\text { N }\end{array}$}} & 40.9 & \\
\hline & & & & & 25.4 & & & & & 109.4 & \\
\hline & & & & & 18.0 & & & & & 76.1 & \\
\hline & & & & & 1.2 & & & & & 5.7 & \\
\hline & & & & & 2 & & & & & 2 & \\
\hline
\end{tabular}




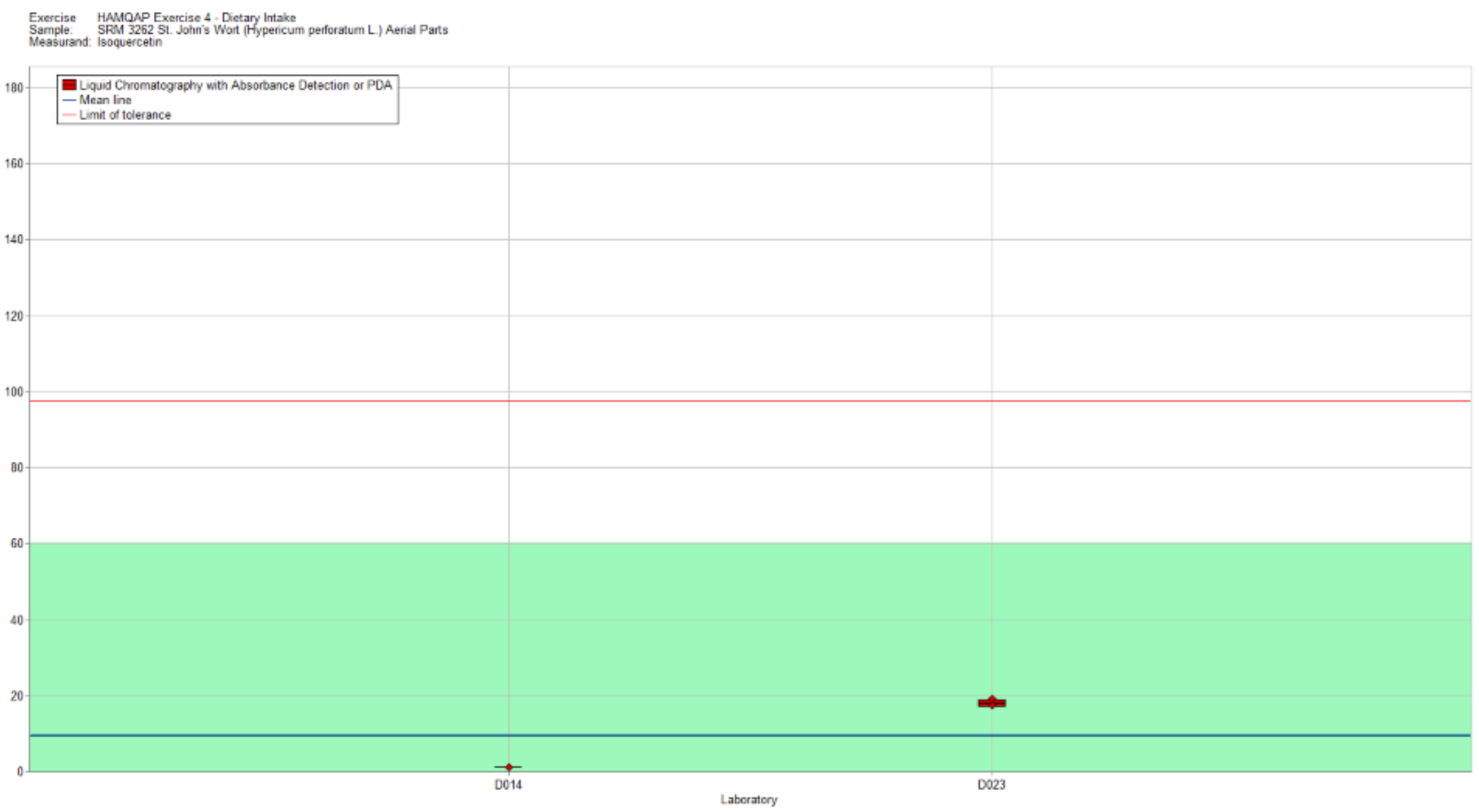

Figure 6-13. Isoquercetin in in SRM 3262 St. John's Wort (Hypericum perforatum) Aerial Parts (data summary view - analytical method). In this view, individual laboratory data are plotted (diamonds) with the individual laboratory standard deviation (rectangle). The solid blue line represents the consensus mean, and the green shaded region represents the $95 \%$ confidence interval for the consensus mean. The solid red line represents the upper consensus range of tolerance, calculated as the values above the consensus mean that result in an acceptable $Z_{\text {comm }}^{\prime}$ score, $\left|Z_{\text {comm }}^{\prime}\right| \leq 2$, with the lower limit set at zero. A NIST value has not been determined in this material. 


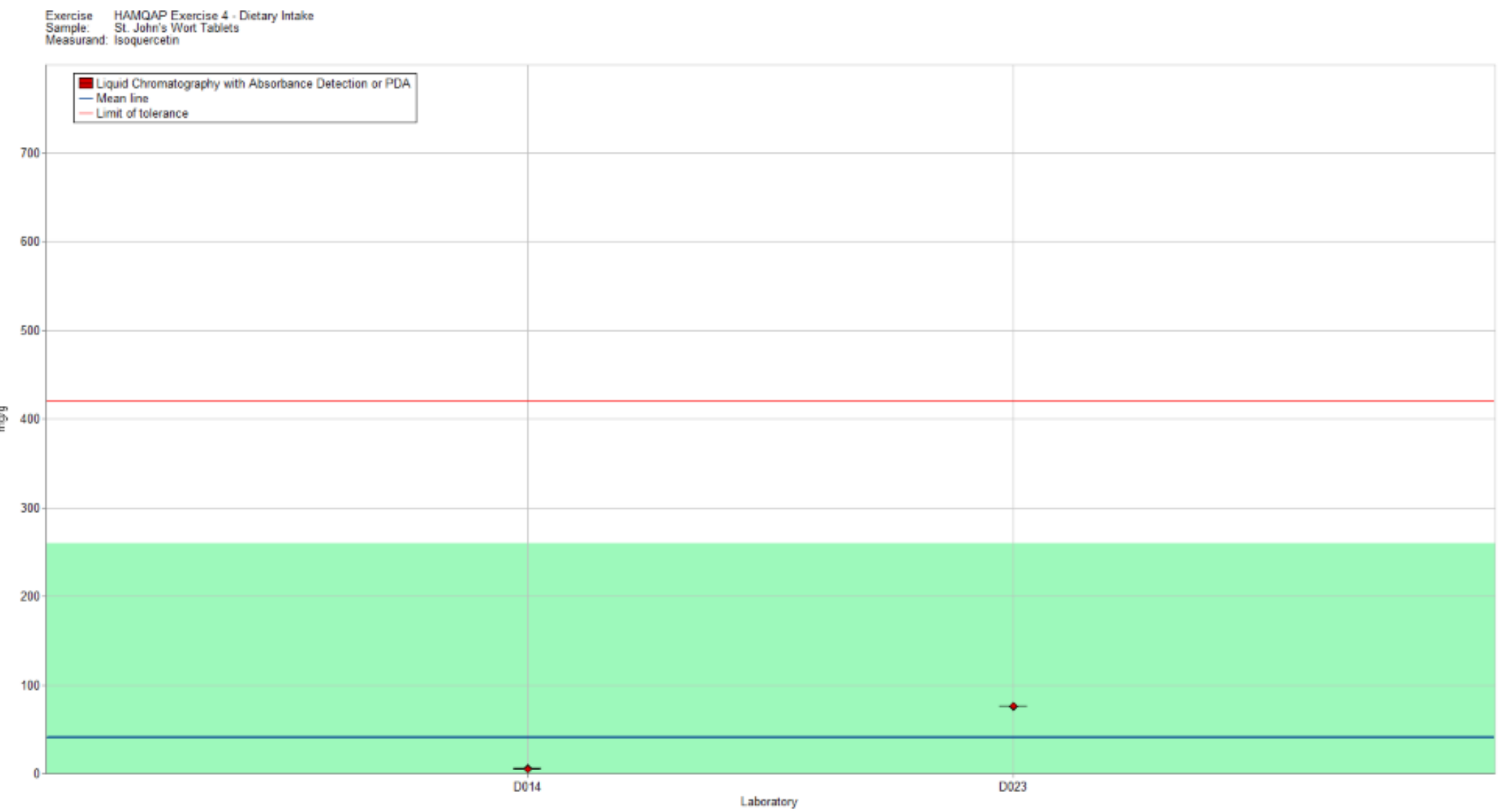

Figure 6-14. Isoquercetin in St. John's Wort Tablets (data summary view - analytical method). In this view, individual laboratory data are plotted (diamonds) with the individual laboratory standard deviation (rectangle). The solid blue line represents the consensus mean, and the green shaded region represents the $95 \%$ confidence interval for the consensus mean. The solid red line represents the upper consensus range of tolerance, calculated as the values above the consensus mean that result in an acceptable $Z_{\text {comm }}^{\prime}$ score, $\left|Z_{\text {comm }}^{\prime}\right| \leq 2$, with the lower limit set at zero. A NIST value has not been determined in this material. 
Table 6-10. Data summary table for quercetin in St. John's Wort. Data points highlighted in red have been flagged as potential outliers (e.g., Grubb and/or Cochran) by the NIST software package.

\begin{tabular}{|c|c|c|c|c|c|c|c|c|c|c|c|}
\hline & & \multicolumn{10}{|c|}{ Quercetin } \\
\hline & & \multicolumn{5}{|c|}{$\begin{array}{l}\text { SRM } 3262 \text { St. John's Wort (Hypericum } \\
\text { perforatum L.) Aerial Parts (mg/g) }\end{array}$} & \multicolumn{5}{|c|}{ St. John's Wort Tablets (mg/g) } \\
\hline & Lab & $\mathbf{A}$ & $\mathbf{B}$ & $\mathbf{C}$ & Avg & SD & $\mathbf{A}$ & $\mathbf{B}$ & $\mathbf{C}$ & Avg & SD \\
\hline \multirow{20}{*}{ 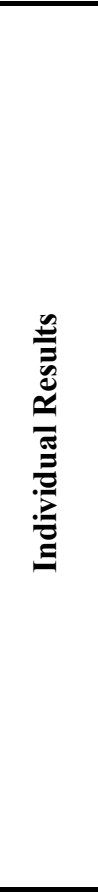 } & Target & & & & & & & & & & \\
\hline & D001 & & & & & & & & & & \\
\hline & D003 & & & & & & & & & & \\
\hline & D004 & 2.49 & 2.52 & 2.55 & 2.52 & 0.03 & 4.53 & 4.65 & 4.62 & 4.60 & 0.06 \\
\hline & D005 & & & & & & & & & & \\
\hline & D007 & & & & & & & & & & \\
\hline & D009 & 0.303 & 0.305 & 0.304 & 0.30 & 0.00 & 2.61 & 2.62 & 2.6 & 2.61 & 0.01 \\
\hline & D010 & & & & & & & & & & \\
\hline & D011 & 9.86 & 9.18 & 9.54 & 9.53 & 0.34 & 22.71 & 23.4 & 23.08 & 23.06 & 0.35 \\
\hline & D014 & 1.7 & 1.6 & 1.7 & 1.67 & 0.06 & 3.34 & 3.45 & 3.15 & 3.31 & 0.15 \\
\hline & D017 & 2 & 2 & 2 & 2.00 & 0.00 & 3.2 & 3.2 & 3.1 & 3.17 & 0.06 \\
\hline & D021 & & & & & & & & & & \\
\hline & D023 & 1.7 & 1.78 & 1.76 & 1.75 & 0.04 & 11.18 & 11.1 & 11.23 & 11.17 & 0.07 \\
\hline & D025 & & & & & & & & & & \\
\hline & D031 & & & & & & & & & & \\
\hline & D033 & 2.45 & 2.51 & 2.48 & 2.48 & 0.03 & 3.44 & 3.51 & 3.3 & 3.42 & 0.11 \\
\hline & D034 & & & & & & & & & & \\
\hline & D046 & & & & & & & & & & \\
\hline & D049 & & & & & & & & & & \\
\hline & D050 & & & & & & 2.41 & 2.64 & 2.57 & 2.54 & 0.12 \\
\hline \multirow{5}{*}{ 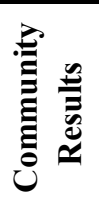 } & & \multirow{5}{*}{\multicolumn{3}{|c|}{$\begin{array}{l}\text { Consensus Mean } \\
\text { Consensus Standard Deviation } \\
\text { Maximum } \\
\text { Minimum } \\
\text { N }\end{array}$}} & 1.79 & & \multirow{5}{*}{\multicolumn{3}{|c|}{$\begin{array}{l}\text { Consensus Mean } \\
\text { Consensus Standard Deviation } \\
\text { Maximum } \\
\text { Minimum } \\
\text { N }\end{array}$}} & 3.27 & \\
\hline & & & & & 0.42 & & & & & 0.57 & \\
\hline & & & & & 9.53 & & & & & 23.06 & \\
\hline & & & & & 0.30 & & & & & 2.54 & \\
\hline & & & & & 7 & & & & & 8 & \\
\hline
\end{tabular}




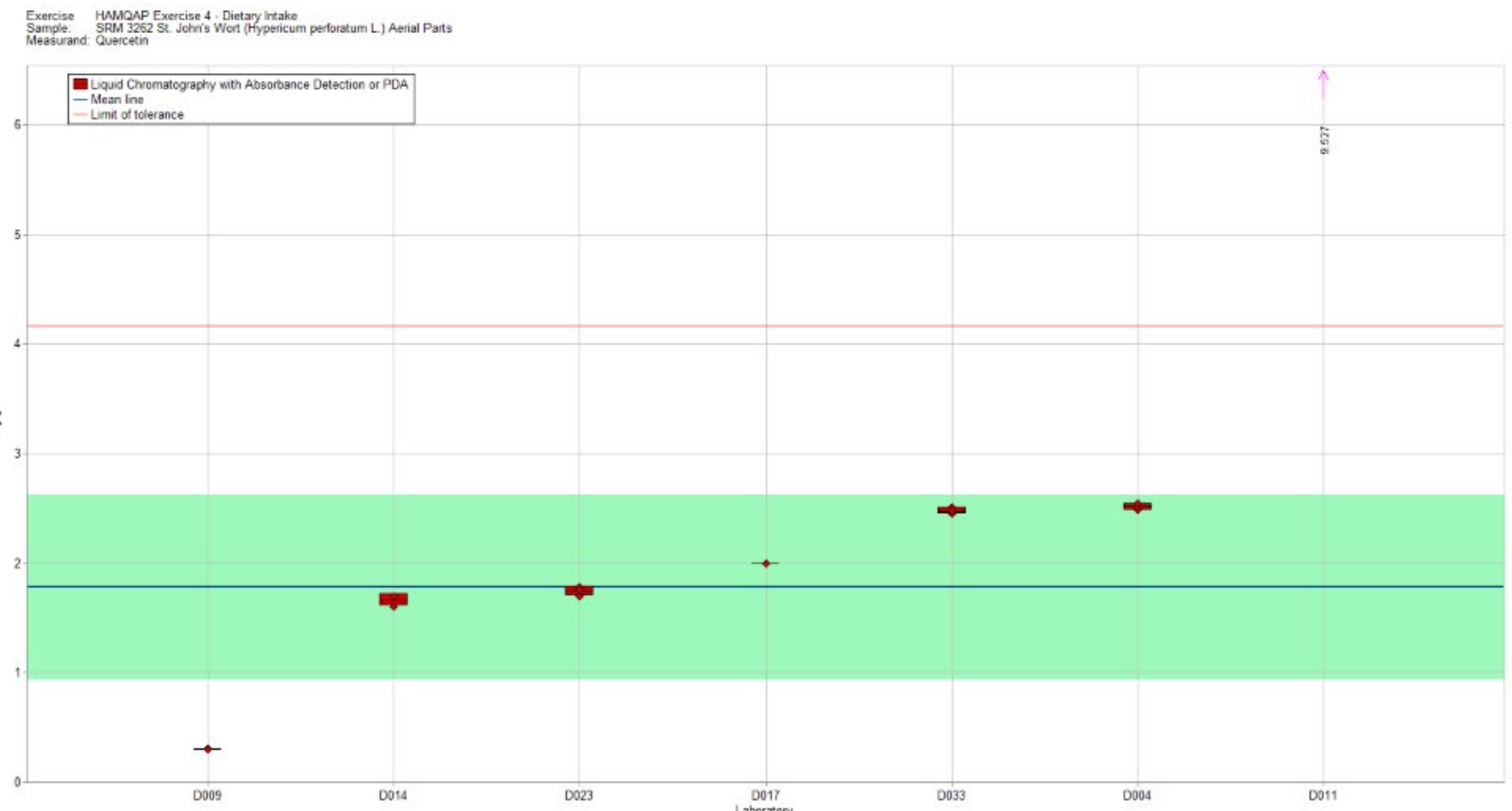

Figure 6-15. Quercetin in SRM 3262 St. John's Wort (Hypericum perforatum) Aerial Parts (data summary view - analytical method). In this view, individual laboratory data are plotted (diamonds) with the individual laboratory standard deviation (rectangle). The solid blue line represents the consensus mean, and the green shaded region represents the $95 \%$ confidence interval for the consensus mean. The solid red line represents the upper consensus range of tolerance, calculated as the values above the consensus mean that result in an acceptable $Z_{\text {comm }}^{\prime}$ score, $\left|Z_{\text {comm }}^{\prime}\right| \leq 2$, with the lower limit set at zero. A NIST value has not been determined in this material. 

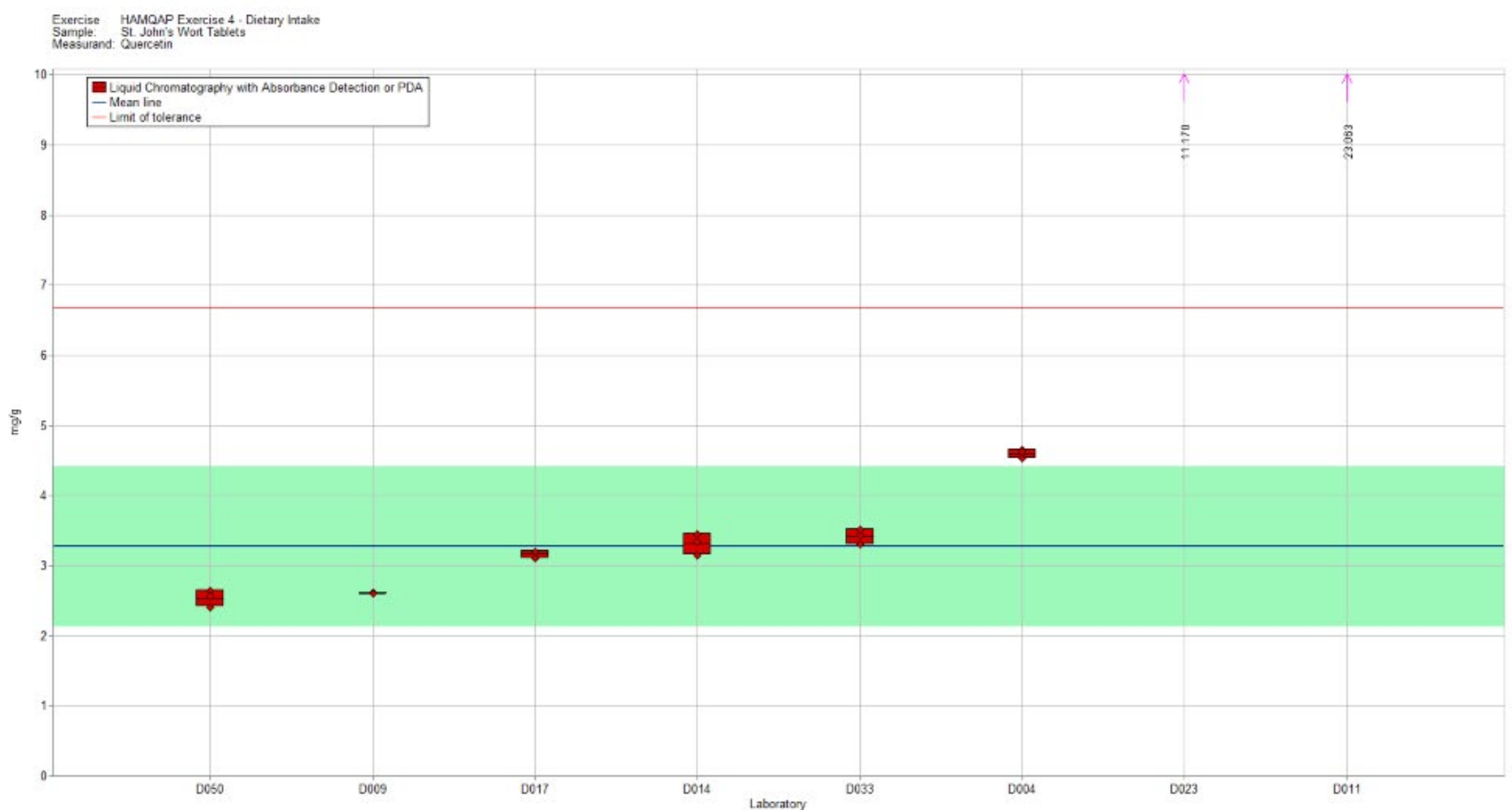

Figure 6-16. Quercetin in St. John's Wort Tablets (data summary view - analytical method). In this view, individual laboratory data are plotted (diamonds) with the individual laboratory standard deviation (rectangle). The solid blue line represents the consensus mean, and the green shaded region represents the $95 \%$ confidence interval for the consensus mean. The solid red line represents the upper consensus range of tolerance, calculated as the values above the consensus mean that result in an acceptable $Z_{\text {comm }}^{\prime}$ score, $\left|Z_{\text {comm }}^{\prime}\right| \leq 2$, with the lower limit set at zero. A NIST value has not been determined in this material. 


\section{SECTION 7: CONTAMINANTS (Nitrate, Nitrite)}

\section{Study Overview}

In this study, participants were provided with samples of SRM 1546a Meat Homogenate and SRM 2385 Slurried Spinach for dietary intake. Participants were asked to use in-house analytical methods to determine the mass fraction $(\mathrm{ng} / \mathrm{g})$ of nitrate and nitrite in each matrix. Nitrites and nitrates are commonly added to foods such as meats as preservatives and to hinder the growth of harmful microorganisms (e.g., Clostridium botulinum). ${ }^{15}$ Nitrates are also used to prevent some cheeses from bloating during fermentation. Nitrate is found naturally in vegetables, with the highest concentrations occurring in leafy vegetables like spinach and lettuce and can enter the food chain through water contaminated from intensive farming methods, livestock production, and sewage discharge. In the body, nitrite and nitrate from food are rapidly absorbed and excreted as nitrate. Some nitrate absorbed by the body is converted by mouth bacteria into nitrite, which can oxidize hemoglobin to methemoglobin and reduce the ability of red blood cells to bind and transport oxygen. In addition, nitrites may also contribute to the formation of carcinogenic nitrosamines. Accurate measurement of nitrate and nitrite in foods and human fluids can inform future risk assessments and assist in determination of safe exposure levels.

\section{Dietary Intake Sample Information}

Meat Homogenate. Participants were provided with one can containing $85 \mathrm{~g}$ of material. Participants were asked to store the material at controlled room temperature, $20{ }^{\circ} \mathrm{C}$ to $25{ }^{\circ} \mathrm{C}$, to use a sample size appropriate for their in-house method of analysis, and to prepare three samples and report three values from the single bottle provided. Before use, participants were instructed to mix the contents of the can thoroughly, taking care to avoid separating fat from the material. One recommended technique is to transfer the entire contents of a can to a plastic bag, then manually squeeze the bag to blend the material. The approximate analyte levels were not reported to participants prior to the study, and target values for nitrate and nitrite in SRM 1546a have not been determined at NIST.

Slurried Spinach. Participants were provided with one jar containing approximately $70 \mathrm{~g}$ of material. Participants were asked to store the material under refrigeration between $2{ }^{\circ} \mathrm{C}$ to $8{ }^{\circ} \mathrm{C}$ in the original unopened jar, to use a sample size appropriate for their in-house method of analysis, and to prepare three samples and report three values from the single jar provided. Before use, participants were instructed to homogenize the contents of the jar using a rotor stator type blender then thoroughly mix the contents. The approximate analyte levels were not reported to participants prior to the study, and target values for nitrate and nitrite in SRM 2385 have not been determined at NIST.

\section{Dietary Intake Study Results}

- Eight laboratories enrolled in this exercise and received samples to measure nitrate and/or nitrite. Four laboratories reported results for each sample (50\% participation).

\footnotetext{
${ }^{15}$ Nitrites and Nitrates Added to Foods. European Food Safety Authority. https://www.efsa.europa.eu/sites/default/files/corporate_publications/files/nitrates-nitrites-170614.pdf (accessed March 2020).
} 
- The variability between the laboratories for nitrate was $12 \%$ in the meat homogenate and $52 \%$ in the slurried spinach. The variability between the laboratories for nitrite was $99 \%$ and over $100 \%$ for meat homogenate and slurried spinach, respectively.

- Laboratories that reported results indicated using solvent extraction paired with either spectrophotometry or ion chromatography, protein precipitation paired with spectrophotometry, dilution paired with LC-absorbance, or an ion selective electrode to measure both analytes.

\section{Dietary Intake Technical Recommendations}

The following general recommendations are offered, as too few data were reported to allow for meaningful specific conclusions to be drawn.

- Any extraction procedure should be optimized to determine the most effective extraction solvent to ensure exhaustive extraction of the analyte from the matrix.

- The optimum number of extraction cycles must be determined by sequential re-extraction of the sample matrix until no further increase in yield is observed. Sequential extractions may be needed if the extraction solvent becomes saturated during the first (or only) extraction cycle.

- "Zero" is not a quantity that can be measured, and therefore a more appropriate result would be to report that a value is below the MDL, LOQ, or QL.

- The use of appropriate calibration materials and quality assurance samples to establish that a method is in control and performing correctly may reduce the likelihood of outlying data. Quality assurance samples can be commercially available reference materials (CRMs, SRMs, or RMs) or materials prepared in-house.

- A linear calibration curve which surrounds the expected sample concentration values should be used for calculations. This curve should include both the lowest and highest expected concentration values of the sample solutions. Extrapolation of results beyond calibration curves may result in incorrect values.

- In general, all results should be checked closely to avoid calculation errors and to be sure that results are reported in the requested units. 
Table 7-1. Individualized data summary table (NIST) for nitrate and nitrite in meat homogenate and slurried spinach.

\section{National Institute of Standards \& Technology}

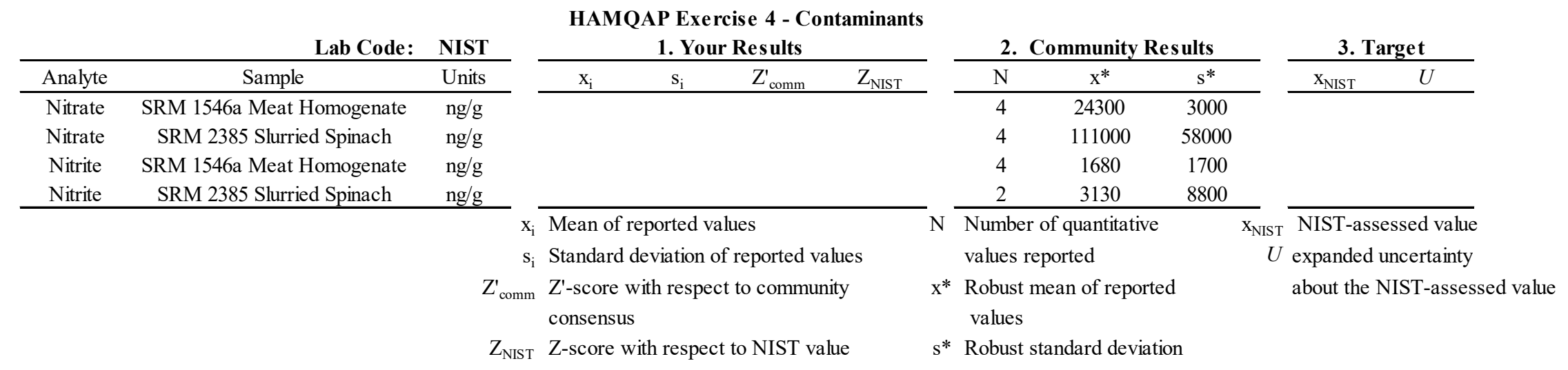


Table 7-2. Data summary table for nitrate in meat homogenate and slurried spinach. Data points highlighted in red have been flagged as potential outliers (e.g., Grubb and/or Cochran) by the NIST software package.

\begin{tabular}{|c|c|c|c|c|c|c|c|c|c|c|c|}
\hline & \multirow[b]{3}{*}{ Lab } & \multicolumn{10}{|c|}{ Nitrate } \\
\hline & & \multicolumn{5}{|c|}{ SRM 1546a Meat Homogenate (ng/g) } & \multicolumn{5}{|c|}{ SRM 2385 Slurried Spinach (ng/g) } \\
\hline & & $\overline{\mathbf{A}}$ & $\mathbf{B}$ & $\mathbf{C}$ & Avg & SD & $\overline{\mathbf{A}}$ & $\mathbf{B}$ & $\mathbf{C}$ & Avg & SD \\
\hline \multirow{9}{*}{ 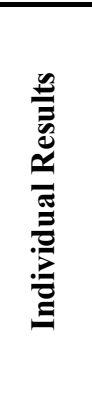 } & Target & & & & & & & & & & \\
\hline & D007 & & & & & & & & & & \\
\hline & D010 & & & & & & & & & & \\
\hline & D020 & & & & & & & & & & \\
\hline & D021 & & & & & & & & & & \\
\hline & D023 & 162138 & 159706 & 180852 & 167565 & 11571 & 182001 & 182047 & 174963 & 179670 & 4077 \\
\hline & D028 & 24183 & 24132 & 24312 & 24209 & 93 & 129723 & 129496 & 129927 & 129715 & 216 \\
\hline & D043 & 28000 & 25000 & 27000 & 26667 & 1528 & 13000 & 21000 & 13000 & 15667 & 4619 \\
\hline & D049 & 21600 & 22600 & 22000 & 22067 & 503 & 122000 & 121000 & 118000 & 120333 & 2082 \\
\hline 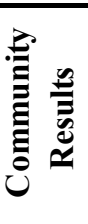 & & $\begin{array}{l}\text { Consenst } \\
\text { Consenst } \\
\text { Maximun } \\
\text { Minimum } \\
\mathrm{N}\end{array}$ & $\begin{array}{l}\text { Mean } \\
\text { Standar }\end{array}$ & Deviation & $\begin{array}{c}24314 \\
2979 \\
167565 \\
22067 \\
4\end{array}$ & & $\begin{array}{l}\text { Consens } \\
\text { Consens } \\
\text { Maximur } \\
\text { Minimun } \\
\mathrm{N}\end{array}$ & $\begin{array}{l}\text { is Mean } \\
\text { is Standarc }\end{array}$ & Deviation & $\begin{array}{c}111346 \\
57819 \\
179670 \\
15667 \\
4\end{array}$ & \\
\hline
\end{tabular}




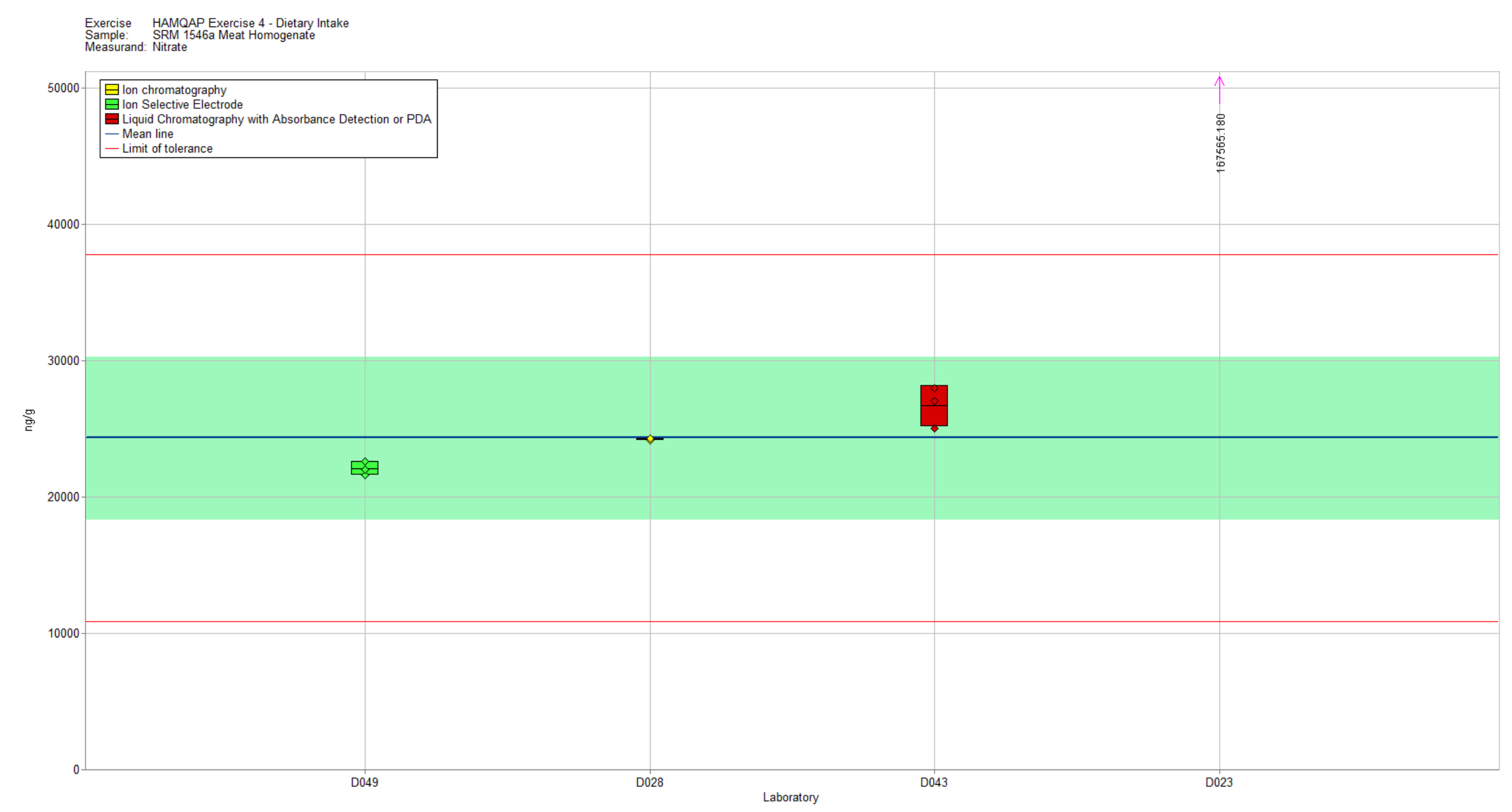

Figure 7-1. Nitrate in SRM 1546a Meat Homogenate (data summary view - analytical method). In this view, individual laboratory data are plotted (diamonds) with the individual laboratory standard deviation (rectangle). The color of the data point represents the analytical method employed. The solid blue line represents the consensus mean, and the green shaded region represents the $95 \%$ confidence interval for the consensus mean. The red solid lines represent the consensus range of tolerance, calculated as the values above and below the consensus mean that result in an acceptable $Z_{\text {comm }}^{\prime}$ score, $\left|Z_{\text {comm }}^{\prime}\right| \leq 2$. A NIST value has not been determined in this material. 


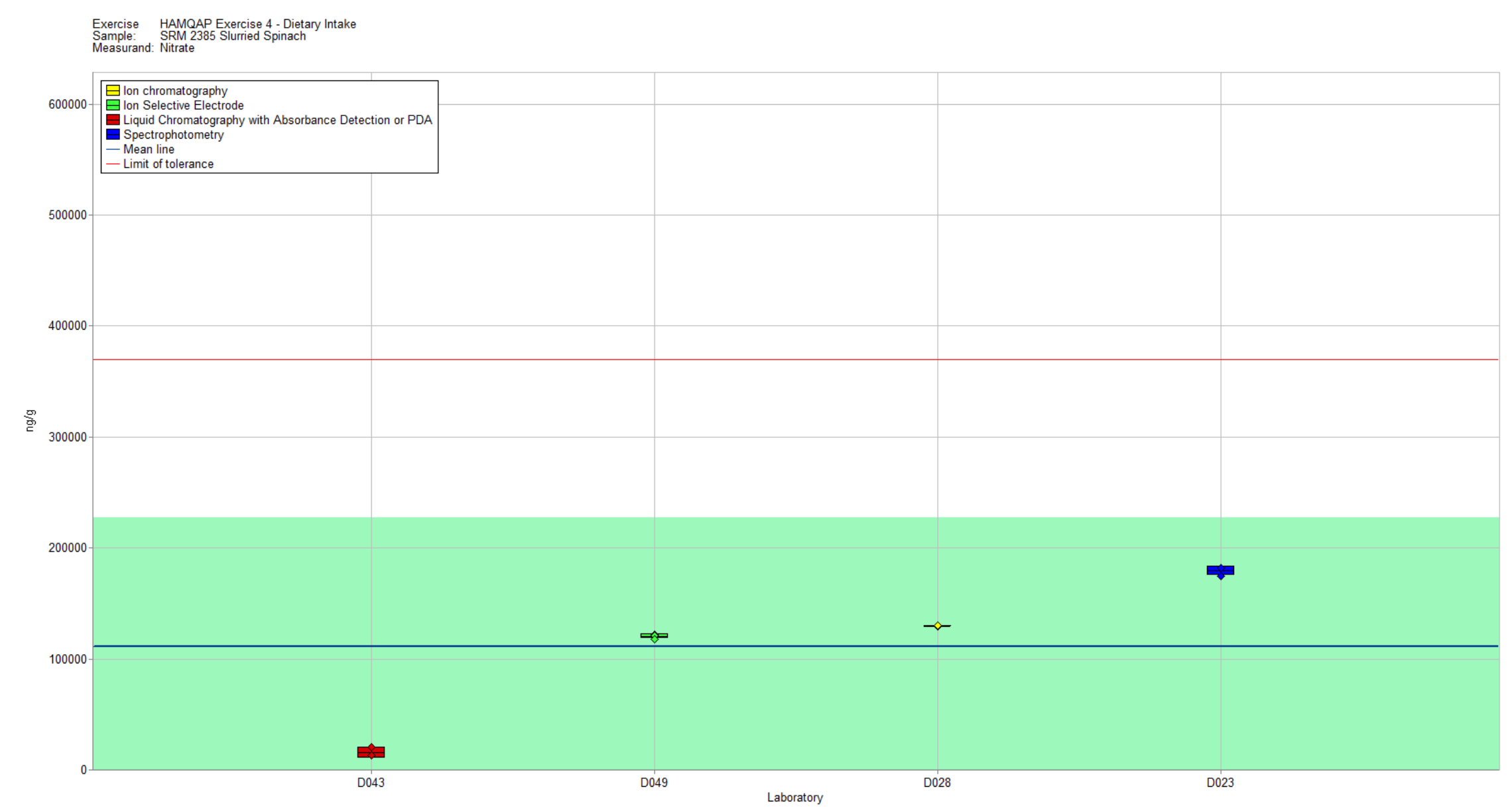

Figure 7-2. Nitrate in SRM 2385 Slurried Spinach (data summary view - analytical method). In this view, individual laboratory data are plotted (diamonds) with the individual laboratory standard deviation (rectangle). The color of the data point represents the analytical method employed. The solid blue line represents the consensus mean, and the green shaded region represents the $95 \%$ confidence interval for the consensus mean. The solid red line represents the upper consensus range of tolerance, calculated as the values above the consensus mean that result in an acceptable $Z_{\text {comm }}^{\prime}$ score, $\left|Z_{\text {comm }}^{\prime}\right| \leq 2$, with the lower limit set at zero. A NIST value has not been determined in this material. 
Table 7-3. Data summary table for nitrite in meat homogenate and slurried spinach. Data points highlighted in red have been flagged as potential outliers (e.g., Grubb and/or Cochran) by the NIST software package.

\begin{tabular}{|c|c|c|c|c|c|c|c|c|c|c|c|}
\hline & \multirow[b]{3}{*}{ Lab } & \multicolumn{10}{|c|}{ Nitrite } \\
\hline & & \multicolumn{4}{|c|}{ SRM 1546a Meat Homogenate (ng/g) } & & \multicolumn{5}{|c|}{ SRM 2385 Slurried Spinach (ng/g) } \\
\hline & & $\mathbf{A}$ & $\bar{B}$ & $\mathbf{C}$ & Avg & SD & $\bar{A}$ & $\mathbf{B}$ & $\mathbf{C}$ & Avg & SD \\
\hline \multirow{9}{*}{ 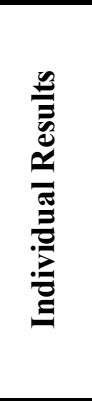 } & Target & & & & & & & & & & \\
\hline & D007 & & & & & & & & & & \\
\hline & D010 & & & & & & & & & & \\
\hline & D020 & & & & & & & & & & \\
\hline & D021 & & & & & & & & & & \\
\hline & D023 & 15294.5 & 15552.5 & 15233.9 & 15360 & 169 & 5963.23 & 5985.01 & 5957.99 & 5969 & 14 \\
\hline & D028 & 600 & 603 & 589 & 597 & 7 & $<500$ & $<500$ & $<500$ & & \\
\hline & D043 & 2293 & 2292 & 2359 & 2315 & 38 & 365 & 273 & 245 & 294 & 63 \\
\hline & D049 & 811 & 788 & 792 & 797 & 12 & $<20000$ & $<20000$ & $<20000$ & & \\
\hline \multirow{5}{*}{ 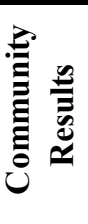 } & & \multicolumn{3}{|c|}{ Consensus Mean } & \multicolumn{2}{|l|}{1684} & \multicolumn{3}{|c|}{ Consensus Mean } & 3132 & \\
\hline & & \multicolumn{4}{|c|}{ Consensus Standard Deviation 1669} & & \multicolumn{3}{|c|}{ Consensus Standard Deviation } & 8810 & \\
\hline & & \multicolumn{2}{|c|}{ Maxımum } & & \multicolumn{2}{|l|}{15360} & \multicolumn{3}{|c|}{ Maximum } & 5969 & \\
\hline & & \multicolumn{2}{|c|}{ Minimum } & & \multicolumn{2}{|l|}{597} & \multicolumn{3}{|c|}{ Minimum } & 294 & \\
\hline & & \multicolumn{2}{|l|}{$\mathrm{N}$} & & \multicolumn{2}{|l|}{4} & \multicolumn{3}{|l|}{$\mathrm{N}$} & 2 & \\
\hline
\end{tabular}




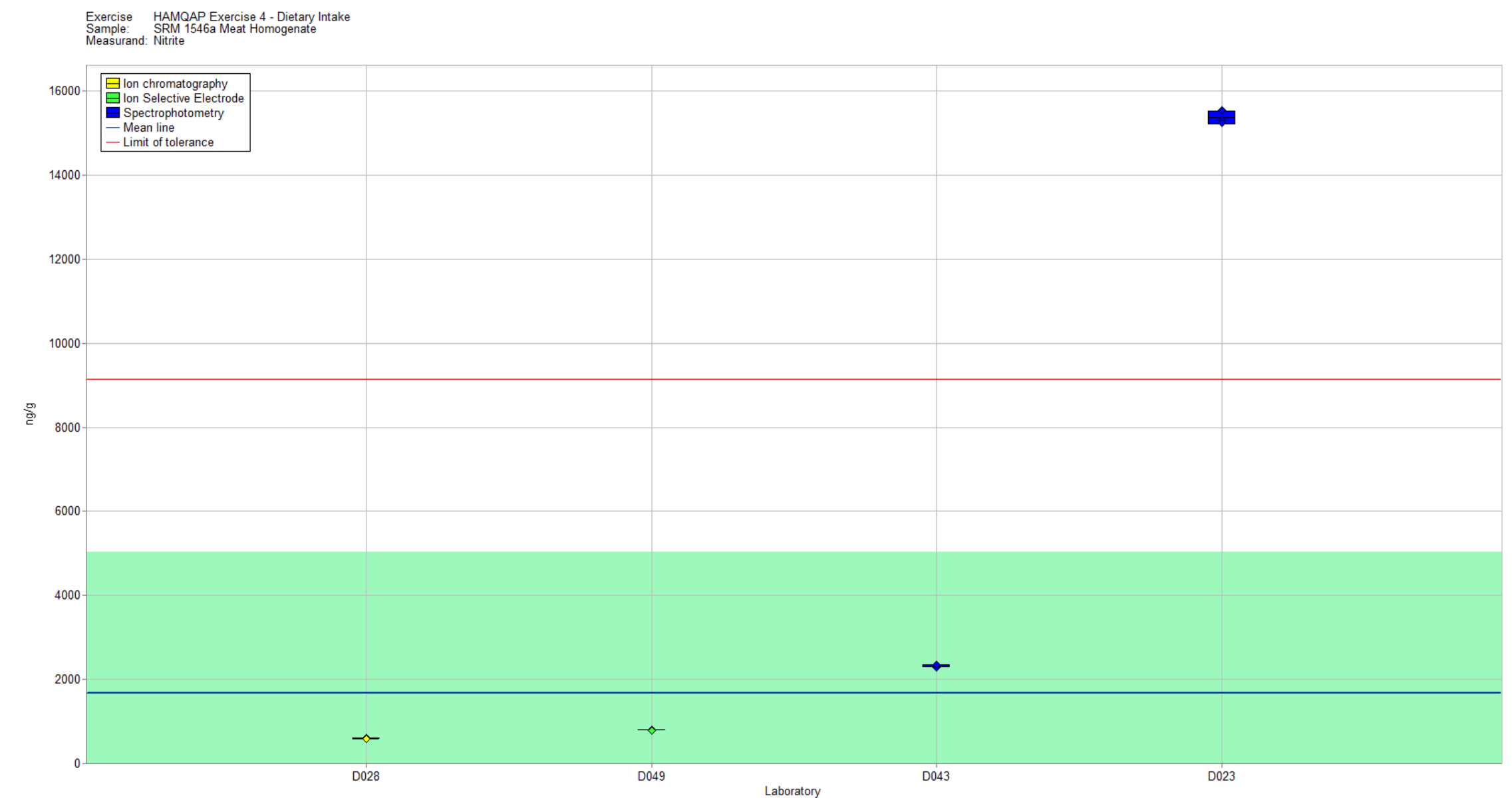

Figure 7-3. Nitrite in SRM 1546a Meat Homogenate (data summary view - analytical method). In this view, individual laboratory data are plotted (diamonds) with the individual laboratory standard deviation (rectangle). The color of the data point represents the analytical method employed. The solid blue line represents the consensus mean, and the green shaded region represents the $95 \%$ confidence interval for the consensus mean. The solid red line represents the upper consensus range of tolerance, calculated as the values above the consensus mean that result in an acceptable $Z_{\text {comm }}^{\prime}$ score, $\left|Z_{\text {comm }}^{\prime}\right| \leq 2$, with the lower limit set at zero. A NIST value has not been determined in this material. 


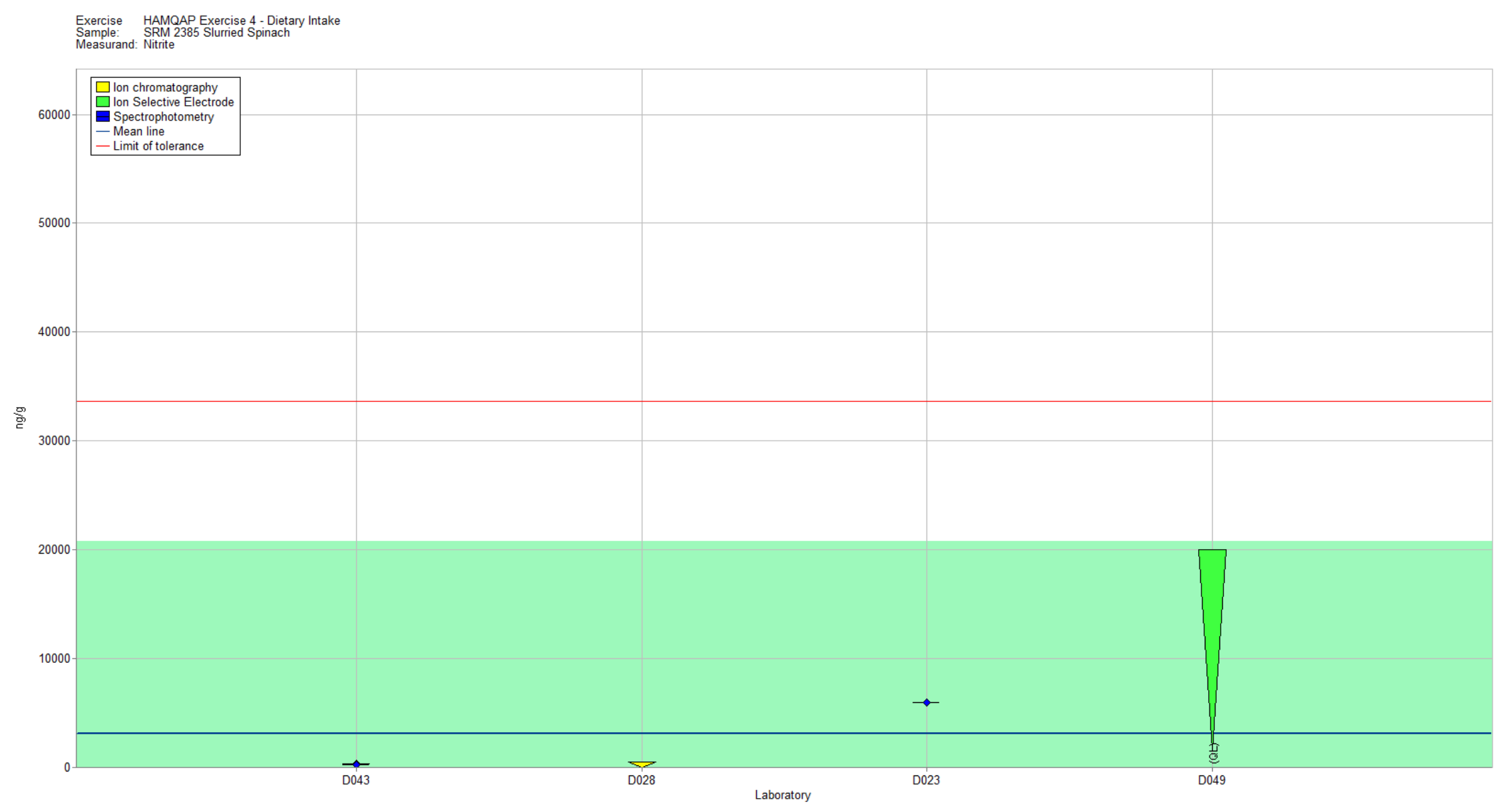

Figure 7-3. Nitrite in SRM 2385 Slurried Spinach (data summary view - analytical method). In this view, individual laboratory data are plotted (diamonds) with the individual laboratory standard deviation (rectangle). The color of the data point represents the analytical method employed. The solid blue line represents the consensus mean, and the green shaded region represents the $95 \%$ confidence interval for the consensus mean. The solid red line represents the upper consensus range of tolerance, calculated as the values above the consensus mean that result in an acceptable $Z_{\text {comm }}^{\prime}$ score, $\left|Z_{\text {comm }}^{\prime}\right| \leq 2$, with the lower limit set at zero. A NIST value has not been determined in this material. 